\title{
Technology-Skill Complementarity in the Early Phase of Industrialization *
}

\author{
Raphaël Franck ${ }^{\dagger}$ and Oded Galor ${ }^{\ddagger}$
}

This Version: February $7^{\text {th }}, 2017$

\begin{abstract}
The research explores the effect of industrialization on human capital formation. Exploiting exogenous regional variations in the adoption of steam engines across France, the study establishes that, in contrast to conventional wisdom that views early industrialization as a predominantly deskilling process, the industrial revolution was conducive for human capital formation, generating broad increases in literacy rates and educational attainment.
\end{abstract}

Keywords: Technology-Skill Complementarity, Economic Growth, Industrialization, Human Capital, Steam Engine.

JEL classification: N33, N34, O14, O33.

*We thank Mario Carillo, Pedro Dal Bo, Martin Fiszbein, Gregory Casey, Marc Klemp, Stelios Michalopoulos, Natacha Postel-Vinay, Assaf Sarid, Yannay Spitzer and David Weil for helpful comments and discussions. We thank Guillaume Daudin, Alan Fernihough, Ömer Özak and Nico Voigtländer for sharing their data with us.

${ }^{\dagger}$ The Hebrew University of Jerusalem, Department of Economics, Mount Scopus, Jerusalem 91905, Israel. Raphael.Franck@mail.huji.ac.il

${ }^{\ddagger}$ Herbert H. Goldberger Professor of Economics, Brown University, Department of Economics, 64 Waterman St, Providence RI 02912 USA. Oded_Galor@brown.edu. 


\section{Introduction}

While it is widely recognized that technology-skill complementarity has characterized the nature of technology in advanced stages of development (Goldin and Katz, 1998), the predominant view had been that the industrial revolution in its early phases was largely a deskilling process (Mokyr, 1993). This deeply entrenched view of the nature of the industrial revolution has been based on anecdotal evidence that has focused nearly exclusively on the adverse effect of factories and the assembly line on artisans. ${ }^{1}$ Nevertheless, as has been the case in technological revolutions that are associated with creative destruction, it is not implausible that the industrial technology generated a demand for new skills while rendering existing ones obsolete.

The research explores the effect of industrialization on human capital formation. In contrast to conventional wisdom that views early industrialization as a predominantly deskilling process, the study establishes that the industrial revolution was conducive to human capital formation, generating broad increases in literacy rates and educational attainment. The research therefore lends further credence to the emerging view that human capital was instrumental in the transition from stagnation to growth (Galor and Weil, 2000; Galor and Moav, 2002; Galor, 2011). ${ }^{2}$

The study utilizes French regional data from the first half of the 19th century to explore the impact of the adoption of industrial technology on human capital formation. It establishes that regions which industrialized earlier experienced a larger human capital formation. Nevertheless, the observed relationship between industrialization and human capital formation may reflect the effect of human capital on the adoption of industrial technology and the persistent effect of pre-industrial characteristics (e.g., economic, institutional and cultural forces) on the joint evolution of industrialization and human capital formation. ${ }^{3}$ Thus, the research exploits arguably exogenous regional variations in the adoption of steam engines across France to assess the effect of industrialization on human capital formation.

Our identification strategy has two components. First, in light of the use of the steam engine in the early phase of industrialization (Mokyr, 1990; Bresnahan and Trajtenberg, 1995; Rosenberg and Trajtenberg, 2004), the study exploits the historical evidence regarding the regional diffusion of the steam engine (Ballot, 1923; Sée, 1925; Léon, 1976) to identify the impact of regional variations in the number of steam engines in 1839-1847 on human capital formation.

\footnotetext{
${ }^{1}$ This deeply entrenched view regarded literacy as largely a cultural skill or a hierarchical symbol with a limited role in the production process in the first stage of industrialization (Mitch, 1992).

${ }^{2}$ The human capital channel is further underlined by Lagerlöf (2003, 2006) Doepke (2004) and Galor and Mountford (2008).

${ }^{3}$ Indeed, human capital appears to have had an effect on development in the pre-industrial era. Boucekkine et al. (2007) demonstrate the importance of literacy in urbanization and the transition from stagnation to growth. Squicciarini and Voigtländer (2015) establish that the upper tail of the human capital distribution in the second half of the 18th century had a positive effect on urbanization and wages in some industrial sectors in the subsequent decades. Furthermore, de la Croix et al. (2016) show the importance of apprenticeship institutions in the emergence of industrialization.
} 
Specifically, it exploits the distances between the administrative center of each French department (the administrative division of the French territory) and Fresnes-sur-Escaut, where a steam engine was first successfully used for industrial purpose in 1732, as exogenous source of variations in industrialization across France. ${ }^{4}$ Second, we consider that the local availability of a technology does not imply that it would be adopted. Therefore we exploit exogenous variation in wheat prices (Labrousse et al., 1970) before 1839 to identify departments which would be more likely to adopt steam engines. We expect that areas where local wheat prices declined more before 1839, i.e., where agriculture became less profitable, were more likely to adopt steam engines.

The study establishes that the number of steam engines in industrial production in the 18391847 period had a positive and significant impact on the formation of human capital in the early stages of the industrial revolution. A larger number of steam engines in a given department in 1839-1847 had a positive and significant effect on the number of teachers in 1840 and 1863, on the share of children in primary schools in 1840 and 1863, on the share of apprentices in the population in 1863, as well as on the share of literate conscripts (i.e., 20-year old men who had to report for military service) over the 1847-1856 and 1859-1868 periods. Industrial technology also had a positive and significant effect on public spending on primary schooling over the 1855-1863 period.

The results of the empirical analysis are robust to the inclusion of a wide array of exogenous confounding geographical and institutional characteristics, as well as for pre-industrial development, which may have contributed to the relationship between industrialization and human capital formation. First, the study accounts for the potentially confounding impact of exogenous geographical characteristics of each French department on the relationship between industrialization and investments in education. It captures the potential effect of these geographical factors on the profitability of the adoption of the steam engine and the pace of its regional diffusion, as well as on productivity and human capital formation, as a by-product of the rise in income rather than as an outcome of technology-skill complementarity. Second, the analysis captures the potentially confounding effects of the location of departments (i.e., latitude, average temperature, average rainfall, border departments, maritime departments, share of carboniferous area in the department, and the distance to Paris) on the diffusion of the steam engine and the diffusion of development (i.e., income

\footnotetext{
${ }^{4}$ An Englishman named John May obtained in 1726 a privilege to operate steam engines that would pump water in the French kingdom, with John Meeres, another Englishman. They set up the first steam engine in Passy (which was then outside but is now within the administrative boundaries of Paris) to raise water from the Seine river to supply the French capital with water. However it appears that this commercial and industrial operation stopped quickly or even never took off. Indeed, when Forest de Bélidor (1737) published his treatise on engineering in 17371739, he mentioned that the steam engine in Fresnes-sur-Escaut was the only one operated in France (see, e.g., Lord (1923) and Dickinson (1939)). Moreover, as established below, the diffusion of the steam engines across the French departments, i.e., the administrative divisions of the French territory created in 1790, is orthogonal to the distances between each department and Paris, the capital and economic center of the country. If anything, this pattern is very similar to what happened in England: Nuvolari et al. (2011) indicate that the first industrial use of the steam engine was in the Wheal Vor tin mine in Cornwall in 1710, but stopped quickly, and that the first successful commercial use of a steam engine took place in 1712 in England, in a coal mine near Wolverhampton (see also Mokyr (1990, p.85)).
} 
and education). Third, the study accounts for the differential level of development across France in the pre-industrial era that may have had a joint impact on the process of industrialization and the formation of human capital. In particular, it takes into account the potentially confounding effect of the persistence of pre-industrial development and the persistence of pre-industrial literacy rates. Finally, the results are robust to the inclusion of additional potentially confounding factors, such as the presence of raw material, measures of early economic integration, past population density and past fertility rates.

The remainder of this article is as follows. Section 2 provides an overview of schooling and literacy in the process of industrialization in France. Section 3 presents our data. Section 4 discusses our empirical strategy. Section 5 presents our main results and Section 6 our robustness checks. Section 7 provides concluding remarks.

\section{Schooling and Literacy in the Process of Industrialization in France}

France was one of the first countries to industrialize in Europe in the 18th century and its industrialization continued during the 19th century. However, by 1914, its living standards remained below those of England and it had been overtaken by Germany as the leading industrial country in continental Europe. The slower path of industrialization in France has been attributed to the consequences of the French Revolution (e.g., wars, legal reforms and land redistribution), the patterns of domestic and foreign investment, cultural preferences for public services, as well as the comparative advantage of France in agriculture vis-a-vis England and Germany (see the discussion in, e.g., Lévy-Leboyer and Bourguignon, 1990; Crouzet, 2003).

\subsection{Schooling in France before and during the Industrial Revolution}

Prior to the French Revolution in 1789, the provision of education in the contemporary French territory was predominantly left to the Catholic Church, reflecting the limited control of the central government and the lack of linguistic unity across the country (Weber, 1976). However, the evolution of state capacity, national identity, and linguistic uniformity over the centuries intensified the involvement of the state in the provision of education while diminishing the role of the church during the 19th century.

\subsubsection{Education Prior to the French Revolution}

Until the rise of Protestantism in the 16th century, the Catholic Church mainly provided education to the privileged members of society (Rouche, 2003). However, the spread of Protestantism, and 
the rise in the emphasis on literacy as a means to understand the Holy Scripture, had altered the attitude of the Catholic Church with respect to the provision of education. The Catholic educational system had progressively become intertwined with its mission of salvation. As such, several religious orders viewed education as their principal mission. The Jesuits had gradually focused their efforts on the education of children from the aristocratic classes while the Frères des Ecoles Chrétiennes (Brothers of Christian Schools) led by Jean-Baptiste de la Salle (1651-1719) sought to provide free education to the masses. Moreover, female religious communities (e.g., Ursulines, Filles de la Charité) provided schooling for girls

The nature of the education provided by the Church over this period was not subjected to interference from the central government. In fact, except for the universities which were controlled by the State from the late 16th century onwards, the various Catholic orders had built an education system which was independent from the French kings. ${ }^{5}$ However, the monopoly of the Church in the provision of education ended abruptly during the French Revolution in 1789.

\subsubsection{Education in the Aftermath of the French Revolution}

The transformation of the French society during the Revolution in 1789 affected the provision of education as well. In particular, article 22 of the Declaration of the Rights of Man and of the Citizen in 1793 explicitly stated that education is a universal right. Nevertheless, the Constitution of the First French Republic (1792-1799) did not underline the role of state-funded secular education. The demise of the Catholic Church (e.g., the confiscation of its property and the imprisonment and execution of priests) during the French Revolution devastated its ability to remain the provider of education, but secular education was nevertheless slow to emerge (Godechot, 1951; Tackett, 1986).

The rise of Napoleon Bonaparte to power (1799-1815) and his interest in maintaining the relationship with Rome, permitted the Church to regain a prominent position in the provision of education in France. ${ }^{6}$ In particular, according to the 17th March 1808 decree on education, the Frères des Ecoles Chrétiennes were left in charge of primary schooling and of training teachers while school curriculum was to be conform to the teachings of the Catholic Church. However this decree also created a secular body - the Université - that was assigned the management of public (secular) education. Throughout the 19th century, the Université would try to counter the Church's influence in the education system (Mayeur, 2003).

After Napoleon's fall in 1815 and the accession to power of King Louis XVIII (1815-1824), from the senior branch of the Bourbon family, strengthened initially the educational monopoly of

\footnotetext{
${ }^{5}$ Nevertheless, some conflicts over the nature of schooling took place between the Jesuits and the Universities as well as between various religious Congregations. In particular, the Jesuits were expelled by King Louis XV in 1764 and their school network was overtaken by the Oratorians.

${ }^{6}$ This state of affairs suited Napoleon Bonaparte because the Concordat (the 1801 treaty which he had signed with Pope Pius VII and which structured the relationship between the French State and the Church), provided him control over the appointment of bishops.
} 
the Church. In particular, the 29 February 1816 law required local priests to certify the morality of primary school teachers. However, after the 1827 parliamentary election of a more liberal government, primary school teachers were placed under the authority of the Université, against the wishes of the Church.

The 1830 Revolution which overthrew King Charles X (1824-1830), Louis XVIII's brother and successor, installed King Louis-Philippe I (1830-1848), from the cadet Orléans branch of the Bourbon family and put in power members of the liberal bourgeoisie who were rather hostile to the Catholic Church. This led Catholics to lobby for an educational network of their own outside the control of the State, under the guise of "freedom of education". Ultimately, François Guizot, King Louis-Philippe I's Prime Minister, enacted the 28 June 1833 law which reshaped schooling in France and enabled the Church to organize its own private education system. In addition, the Church retained its influence over the curriculum of public schools (e.g., religious instruction remained mandatory while the Frères des Ecoles Chrétiennes were often employed as teachers in public schools). The organization of secondary schooling then became the main point of contention between the Church and its opponents, and it was only after the fall of Louis-Philippe I in 1848 and the establishment of the Second Republic (1848-1851) that the Church was allowed to organize its own network of secondary schools while obtaining subsidies from the State and local governments (15 March 1850 law enacted by Education minister Alfred de Falloux). Moreover towns were not compelled to fund a public primary school if there was already a private (i.e., Catholic) school in their jurisdiction, and teachers had to fulfill the religious duties prescribed by the Church (27 August 1851 regulation).

Interestingly enough, technical education was less of a battleground between the State and the Church than general primary schooling. This might have been due to the lesser importance of technical education in a period where training on the job was widespread. Nonetheless the 28 June 1833 law which reshaped schooling in France also established "schools of higher primary education" that provided the basics of technical education (Marchand, 2005). But it took another 18 years before the 22 February 1851 law formally established schools for apprentices. Still, a decade later, few students attended these technical schools and most of those who did were enrolled in public schools, not in religious schools (Ministère De l'Instruction Publique, 1865). Conversely, in the 1850s and early 1860s, enrollment in Catholic primary schools, especially for girls, was growing at the expense of enrollment in public primary schools. This led Victory Duruy, the education minister of Napoleon III (1851-1870) after 1863, to counter the decline in public schooling, thereby initiating a conflict between Catholics and secular politicians which would reach its climax after the establishment of the Third Republic. 


\subsubsection{Education From the Establishment of the Third Republic to World War I}

Following the demise of the Napoleon III's Empire in 1870 and the establishment of Third Republic (1875-1940), France became divided between Republicans and Monarchists. The latter received most of their support from the Catholics who associated the Republicans with the 1789 French Revolution and the anti-religious policies of the revolutionaries. This political stance was shared by the clergy and the laity, as well as by liberal and intransigent Catholics alike. But the Catholic opposition to the Republic was matched by the Republicans' hostility to the Church and their determination to turn France into a more secular society (Franck, 2016).$^{7}$ In particular, in an attempt to crowd out Catholic schooling, the Republicans increased spending on primary schooling by the central state in the 1880-1890 period. Moreover, in 1881 and 1882, the Republicans passed laws promoting free, secular and mandatory education until age $13 .{ }^{8}$ However enrollment in Catholic schools, especially in primary schools for girls, remained high (Mayeur, 1979).

At the turn of the 20th century, the Republicans realized that their attempt to crowd out the schooling system of the Church had failed and used their legal power to renew their attacks (Franck and Johnson, 2016). They passed the 1 July 1901 law which, de facto, prevented monks and nuns from teaching, thereby forcing many Catholic schools to close. Four years later, the Republicans separated Church and State (Franck, 2010): the French state protected freedom of conscience but stopped recognizing official religions and ended subsidies to religious groups. In theory, Catholic schools had become private institutions outside the scope of the French government's reach. In practice, however, the Republicans wanted to control the curricula of Catholic schools. This would be the main point of contention between Republicans and Catholics until World War I. Thus the bishops' opposition in 1909 to the imposition by the State of governmental manuals led Republicans to rally around the "defense of secular education". They passed additional laws pertaining to public schooling attendance and enabled prosecutions against priests who instructed parents not to enroll their children in state-funded secular schools. After World War I, political debates dealing with private religious schooling and public secular schooling have periodically resurfaced in France. However they did not stir passions as much as in the 1870-1914 period.

\section{$2.2 \quad$ Literacy Rates in France}

The evolution of literacy and its distribution across French department is rather notable in the course of industrialization. In 1686-1690, prior to the onset of the industrial revolution in France,

\footnotetext{
${ }^{7}$ For instance, the 27 July 1882 law re-legalized divorce.

${ }^{8}$ Before the 20 June 1881 law, all parents but the poorest ones who wanted to enroll their children in school had to pay fees called rétribution scolaire which had been established by the 3 Brumaire An IV (25 October 1795) law. The 20 June 1881 law reestablished free education, which had been first instituted by decrees of the Convention during the French Revolution but had been reversed by the 3 Brumaire An IV law. It should be noted that by the 1870 s, the rétribution scolaire only remained significant in rural areas and had been replaced by local taxes in urban areas.
} 


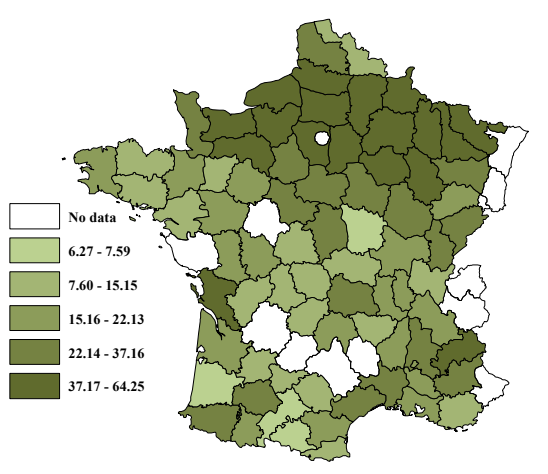

A. Literacy rates in 1686-1690.

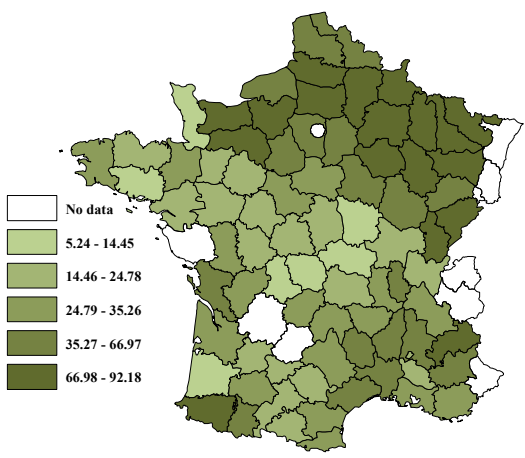

B. Literacy rates in $1786-1790$.

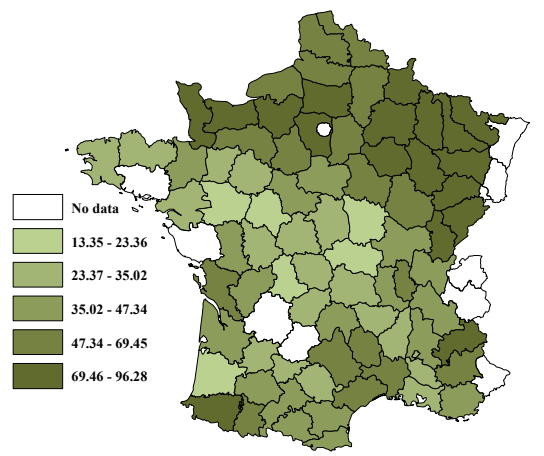

C. Literacy rates in $1816-1820$. Note: Literacy is captured by the share of grooms who signed their marriage license during each period.

Figure 1: Pre-industrial literacy rates of French departments

$25.9 \%$ of grooms could sign their names, reflecting substantial variations in literacy across France as depicted in Panel A of Figure $1 .^{9}$ In particular, literacy rates were higher in the regions in the North and East of France. ${ }^{10}$

Literacy rates had steadily increased in the subsequent century and $42 \%$ of grooms could sign their names in 1786-1790 and 50.61\% in 1816-1820, in spite of the Revolutionary and Napoleonic wars. As depicted in Panels B and $\mathrm{C}$ of Figure 1 regional variations across France remained and the domination of the Northern and the Eastern regions persisted. However, literacy rates in some departments had evolved faster than in others, notably in the South (e.g., Aveyron) and the South East along the Mediterranean Sea (Bouches du Rhône, Var). Moreover, the potential association between industrialization and literacy is rather apparent. In particular, Aveyron, Bouches du Rhône and Var were among the most industrialized departments in the South of France.

The increase in literacy rates continued throughout the 19th century so that the share of French conscripts (i.e., 20-year old men reporting for military service in the department where their father lived) who could read and write grew from $54.27 \%$ in 1838 to $84.83 \%$ in 1881 . Thus, a significant fraction of Frenchmen were literate even before the adoption of the 1881-1882 laws on mandatory and free public schooling (Diebolt et al., 2005).

\footnotetext{
${ }^{9}$ Data on literacy in France before the mid-19th century is scarce and incomplete. There is however data on the number of Frenchmen who could sign their marriage license in 1686-1690, 1786-1790 and 1816-1820 (Furet and Ozouf, 1977).

${ }^{10}$ For a discussion of the cultural, religious and economic factors which potentially explained the regional differences in the share of literate grooms, see notably Furet and Ozouf (1977), Grew and Harrigan (1991) and Diebolt et al. (2005).
} 


\section{Data and Main Variables}

This section examines the evolution of industrialization and human capital formation across the 85 mainland French departments, based on the administrative division of France in the 1839-1847 period, accounting for the geographical and the institutional characteristics of these regions. The initial partition of the French territory in 1790 was designed to ensure that the travel distance by horse from any location within the department to the main administrative center would not exceed one day. The initial territory of each department was therefore orthogonal to the preindustrial wealth levels and literacy rates while the subsequent minor changes in the borders of some departments reflected political forces rather than the effect of industrialization, the adoption of the steam engine and human capital formation. Table A.1 reports the descriptive statistics for the variables in the empirical analysis across these departments.

\subsection{Measures of Human Capital}

The study explores the effect of industrialization on the evolution of human capital in the early stages of the industrial revolution. It takes advantage of the industrial survey conducted between 1839 and 1847 to assess the short-run impact of industrialization across France on several indicators of human capital accumulation.

\subsubsection{Teachers, Pupils and Apprentices}

The impact of early industrialization on human capital during the early phase of the industrial revolution is assessed by the effect of the differential level of industrialization across France on the number of teachers, pupils and apprentices in each department. ${ }^{11}$

First, the research examines the effect of industrialization on the number of teachers in each department. Surveys undertaken in 1840 and 1863 by the French bureau of statistics (Statistique Générale de la France) indicate that the average number of teachers per department rose from 742 in 1840 to 1243 in 1863 . The surveys also show that there was considerable variation in the number of teachers across departments.

Second, the study explores the impact of industrialization on the number of pupils enrolled in the primary schools of each department (per 10,000 inhabitants). Surveys carried out in 1840 and 1863 by the French bureau of statistics (Statistique Générale de la France) show that the average number of pupils in each department (per 10,000 inhabitants) grew from 874 in 1840 to 1179 in 1863, with substantial variation in the number of pupils across France.

\footnotetext{
${ }^{11}$ The effect of industrialization on the pupils-to-teacher ratio is not necessarily indicative of the effect on human capital formation. In the face of resource constraints, changes in this ratio may reflect the local decision-makers' view about the trade-off between the quality and the quantity of education.
} 
Third, the research analyzes the effect of industrialization on technical education as captured by the number of males enrolled in apprentice schools (per 10,000 inhabitants). The data (Ministère De l'Instruction Publique, 1865) show that in 1863, the average number of apprentices in each department (per 10,000 inhabitants) was equal to 2.71 and was therefore an order of magnitude smaller than the number of pupils in primary schools.

\subsubsection{Literacy}

The impact of early industrialization on literacy during the first phase of the industrial revolution is captured by its effect on the share of French army conscripts (i.e., 20-year-old men who reported for military service in the department where their father lived) who could read and write. The analysis focuses on the share of literate conscripts over the 1859-1868 decade, i.e., individuals who were born between 1839 (when the industrial survey began) and 1848 (a year after the survey was completed). ${ }^{12}$ As reported in Table A.1, $74.0 \%$ of the French conscripts were literate over the 1859-1868 period.

\subsection{Steam Engines}

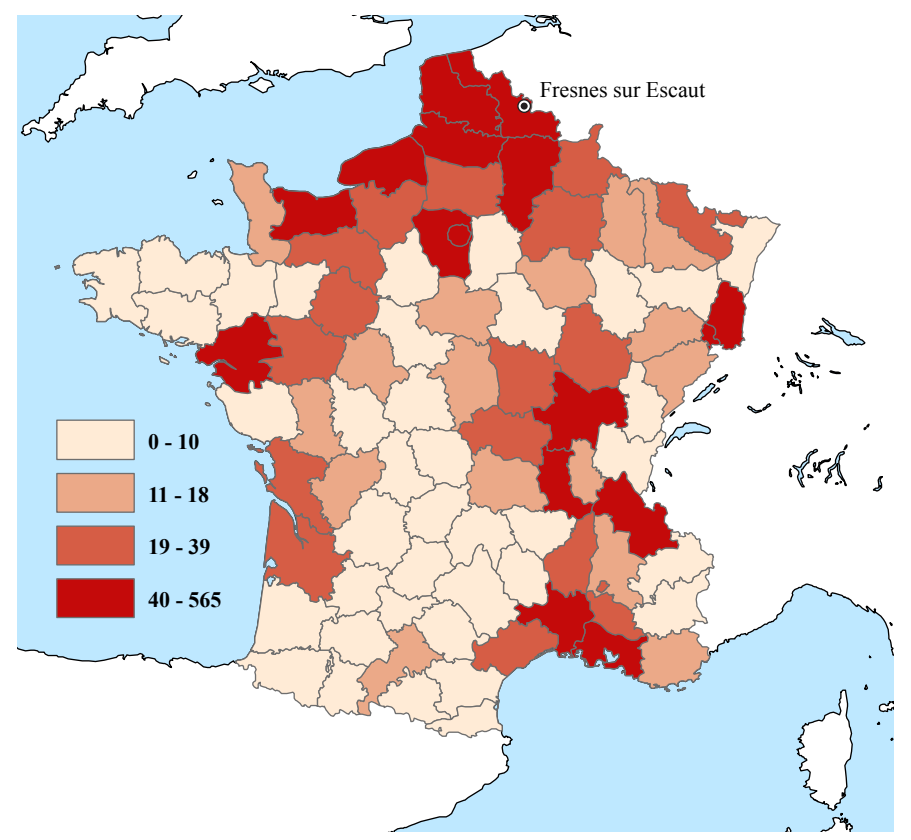

Figure 2: The distribution of the number of steam engines across departments in mainland France, 1839-1847.

The research explores the effect of the introduction of industrial technology on human capital.

\footnotetext{
${ }^{12}$ As a robustness check, we explore in the Appendix the impact of industrialization on the literacy rate of French conscripts over the 1847-1856 decade: these 20-year old men were born between 1827 and 1836 (i.e., three years before the industrial survey began).
} 
In light of the pivotal role played by the steam engine during the first industrial revolution, it exploits variations in the industrial use of the steam engine across France. Specifically, the analysis focuses on the number of steam engines used in each French department as reported in the industrial survey carried out by the French bureau of statistics (Statistique Générale de la France) between 1839 and 1847 (see Chanut et al. (2000) for a discussion.) As discussed by Chanut et al. (2000), the survey was begun in 1839 and almost completed in 1841 when it was halted under popular pressure, amid growing fears that the survey data would be used to support the fiscal reforms supporters by then finance minister Georges Humann. It was in 1844 that the central government restarted the survey which was eventually completed in 1847. It is however unclear whether local administrators began data collection anew in 1844 or simply sent to the central administration in Paris the data collected between 1839 and 1841 with minor or no updates.

As shown in Figure 2, and analyzed further in the discussion of the identification strategy in Section 4, the distribution of the steam engines across French departments in 1839-1847 suggests a regional pattern of diffusion from Fresnes-sur-Escaut (in the Nord department, at the northern tip of continental France) where the first steam engine in France was successfully used for industrial purposes in 1732. The largest number of steam engines was indeed in the northern part of France. There were fewer in the east and in the south-east, and even less so in the south-west. ${ }^{13}$ Seven departments had no steam engine in 1839-1847 (i.e., Cantal, Côtes-du-Nord, Creuse, Hautes-Alpes, Haute-Loire, Lot and Pyrénées-Orientales). The potential anomalies which are associated with these departments, and in particular regarding the distance of these departments from the threshold level of development which enables the adoption of steam engines, are accounted for by the introduction of a dummy variable that singles them out.

In Table A.2, we report descriptive statistics for the number of steam engines in each of the 16 sectors listed in the 1839-1847 survey: ceramics, chemistry, clothing, construction, food, furniture, leather, lighting, luxury goods, metal objects, metallurgy, mines, sciences \& arts, textile, transportation and wood. The data show that the five sectors with the largest average horse power from steam engines per department are textile, food, mines, metallurgy and metal objects. In this respect, we note that the textile sector had the largest number of steam engines of all the sectors: there were twice as many steam engines in textile than in the food industry and three times more than in the mining sector. Moreover the descriptive statistics on the number of workers in each of the 16 sectors reported in Table A.2 indicate that the chemistry and wood sectors had a larger

\footnotetext{
${ }^{13}$ Seven departments had no steam engine in 1839-1847 (i.e., Cantal, Côtes-du-Nord, Creuse, Hautes-Alpes, Haute-Loire, Lot and Pyrénées-Orientales). In regressions which are available upon request, potential anomalies associated with these departments, and in particular regarding the distance of these departments from the threshold level of development that permits the adoption of steam engines, are accounted for by the introduction of a dummy variable that singles them out. The introduction of this dummy variable does not modify the results which are reported below.
} 
ratio of steam engines per worker, most likely because the textile sector employed many individuals whose work did not require steam engines.

The distribution of steam engines in 1839-1847, teachers in 1840, pupils in 1840, apprentices in 1863 and literate conscripts in 1859-1868 across French departments is depicted in Figure 3.

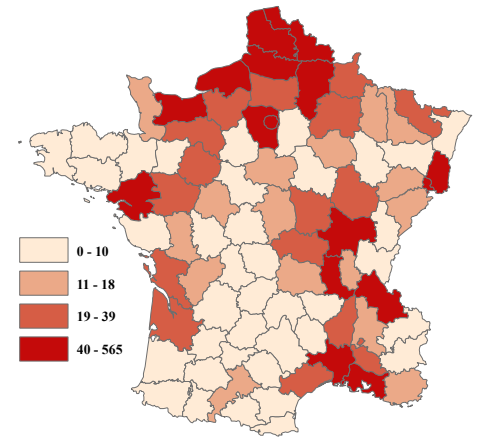

Number of steam engines, 1839-1847.

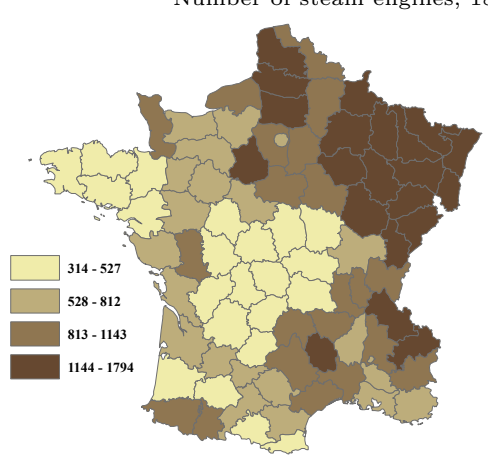

Share of pupils in the population, 1840

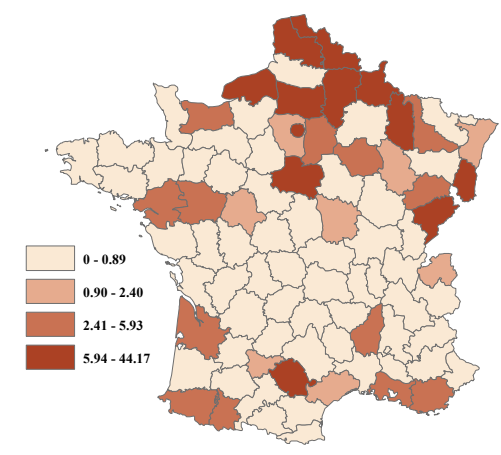

Share of apprentices in the population, 1863.

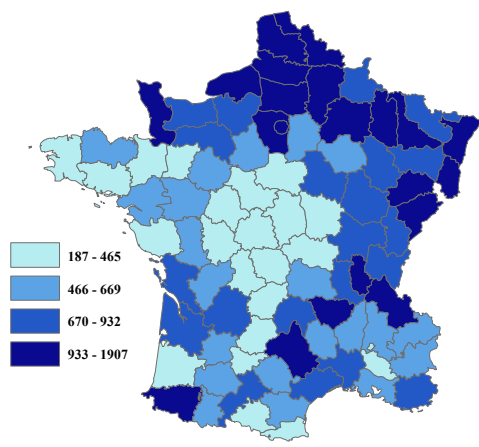

Number of teachers, 1840 .

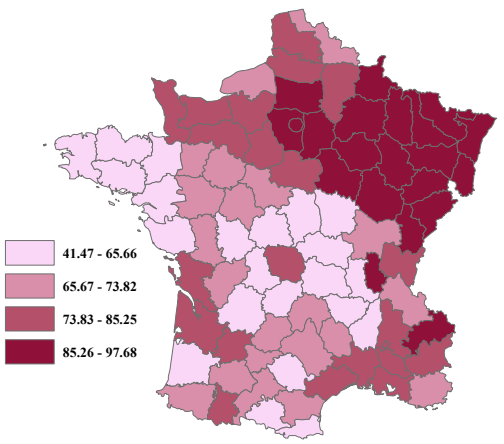

Share of literate conscripts, 1859-1868.

Figure 3: The distribution of steam engines in 1839-1847, teachers in 1840, pupils in 1840, apprentices in 1863 and literate conscripts in 1859-1868 across French departments.

\subsection{Confounding Characteristics of the Departments}

The empirical analysis accounts for observable exogenous confounding geographical and institutional characteristics of each department, as well as for their pre-industrial development, which may have contributed to the relationship between industrialization and human capital formation. Geography may have impacted agricultural productivity as well as the pace of industrialization, and thus income per capita and investments in education. Institutions may have affected jointly the process of industrialization and the increase in literacy. Besides, geographical and institutional factors may have affected human capital indirectly by governing the speed of the diffusion of steam engines across departments. Finally, pre-industrial development may have affected the onset of industrialization and may have had an independent persistent effect on human capital formation. 


\subsubsection{Geographic characteristics}

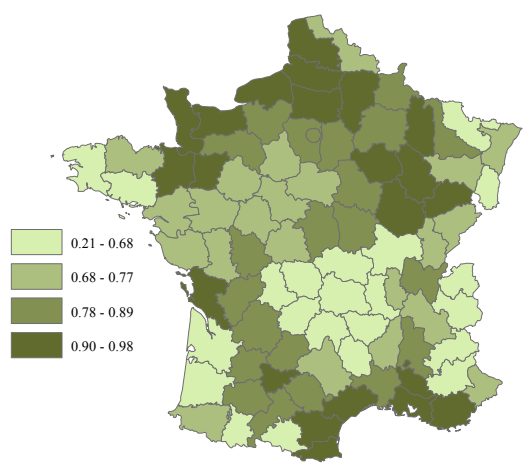

Land Suitability.
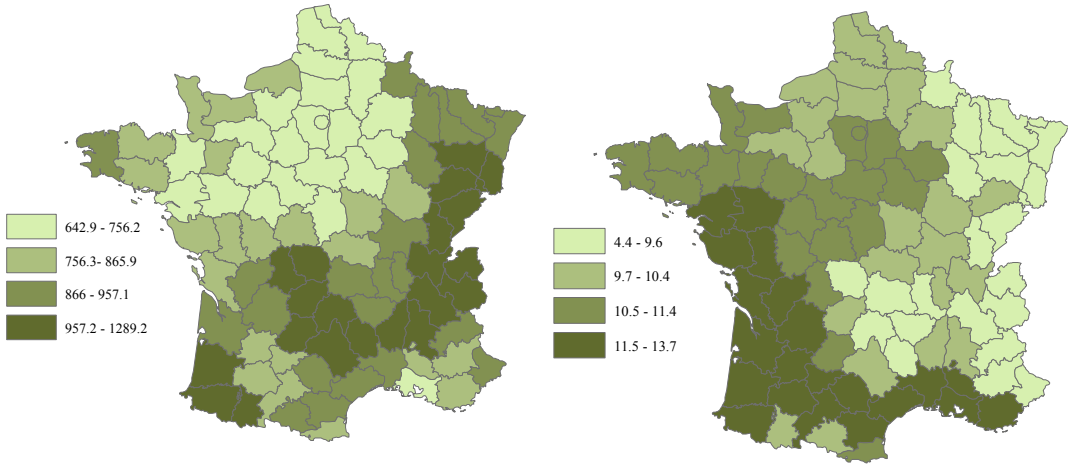

Average Rainfall.

Figure 4: Geographic characteristics of French departments

The empirical analysis takes into account the potentially confounding impact of the exogenous geographic characteristics of each French department (Figure 4) on the relationship between industrialization and human capital. Specifically, it captures the potential effect of these geographical factors on the profitability of the adoption of the steam engine, on the pace of its regional diffusion and thus, on human capital accumulation during the first stages of the industrial revolution.

First, the study accounts for climatic and soil characteristics of each department (i.e., land suitability, average temperature, average rainfall, and latitude (Ramankutty et al., 2002)) that could have affected natural land productivity and therefore, the feasibility and profitability of the transition to the industrial stage of development, as well as income per capita and human capital in each department. Besides, the diffusion of the steam engine across France could have been affected by the presence of raw material required for industrialization. Our regressions thus account for the share of carboniferous area in each department (Fernihough and O'Rourke, 2014).

Second, the analysis captures the confounding effect of the location of each department on the diffusion of development from nearby regions or countries, as well as its effect on the regional diffusion of the steam engine. Namely, it accounts for the effect of the latitude of each department, and maritime departments (i.e., positioned along the sea shore of France) on the pace of this diffusion process.

Finally, the research accounts for the potential differential effects of international trade on the process of development as well as on the adoption the steam engine. In particular, it captures the potential effect of maritime departments (i.e., those departments that are positioned along the sea shore of France), via trade, on the diffusion of the steam engine and thus on economic development as well as its direct effect on human capital formation over this time period. 


\subsubsection{Institutional Characteristics}

Since the empirical analysis focuses on the impact of variations in the adoption of the steam engine on human capital formation across French departments, it ensures that institutional factors which were unique to France as a whole over this time period are not the source of the differential pattern of human capital across these regions. Nevertheless, one region of France over this time period had a unique exposure to institutional characteristics that may have contributed to the observed relationship between industrialization and literacy.

The emergence of state centralization in France and the concentration of political power in Paris before the industrial revolution may have had a differential impact on the political culture and economic prosperity in Paris and its suburbs (i.e., Seine, Seine-et-Marne and Seine-et-Oise). Hence, the analysis includes a dummy variable for these three departments to control for their potential confounding effects on the observed relationship between industrialization and human capital. Moreover, the analysis accounts for the effect of the aerial distance between the administrative center of each department and Paris, thus capturing the potential decline in the reach of the central government in regions at a greater distance from Paris as well as the diminished potential diffusion of development into these regions.

\subsubsection{Pre-industrial Development}

The empirical analysis accounts for the potentially confounding effects of the level of development in the pre-industrial period. The differential level of development across France in the pre-industrial era may have indeed affected jointly the process of development and human capital formation. Namely, it may have affected the adoption of the steam engine and it may have generated, independently, a persistent investment on education. First, the early level of development, as captured by the degree of urbanization (i.e., population of urban centers with more than 10,000 inhabitants) in each French department in 1700 as shown in Panel A of Figure 5 (Lepetit, 1994), may have persisted independently of the process of industrialization. ${ }^{14}$ Second, the number of universities in 1700 in each department as shown in Panel B of Figure 5 (Bosker et al., 2013) may have affected the adoption of the steam engine while contributing to human capital formation independently of the process of industrialization. Third, early literacy rates, as captured by the share of grooms who could sign their marriage license over the 1686-1690, 1786-1790 and 1816-1820 periods as mapped in Figure 1 (Furet and Ozouf, 1977), ${ }^{15}$ may have affected the adoption of the steam engine while contributing to human capital accumulation independently of the process of industrialization.

\footnotetext{
${ }^{14}$ As we discuss below in Section 6 , the qualitative analysis remains intact if the potential effect of past population density is accounted for.

${ }^{15}$ Some observations are missing for these variables. For the $1686-1690$ period, there are no observations for Aveyron, Bas-Rhin, Dordogne, Indre-et-Loire, Lot, Lozère, Haut-Rhin, Lot, Seine and Vendée. For the 1786-1790 period, observations are missing for for Bas-Rhin, Dordogne, Haut-Rhin, Lot, Seine and Vendée. For the 1816-1820
} 


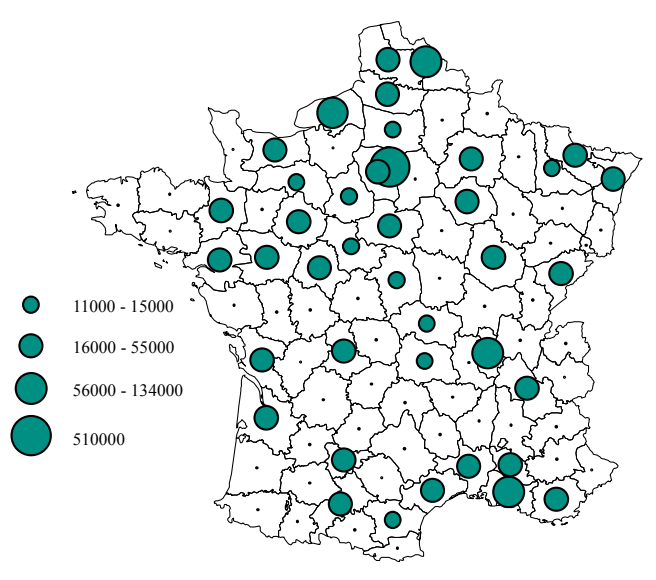

A. Urban population in 1700 .

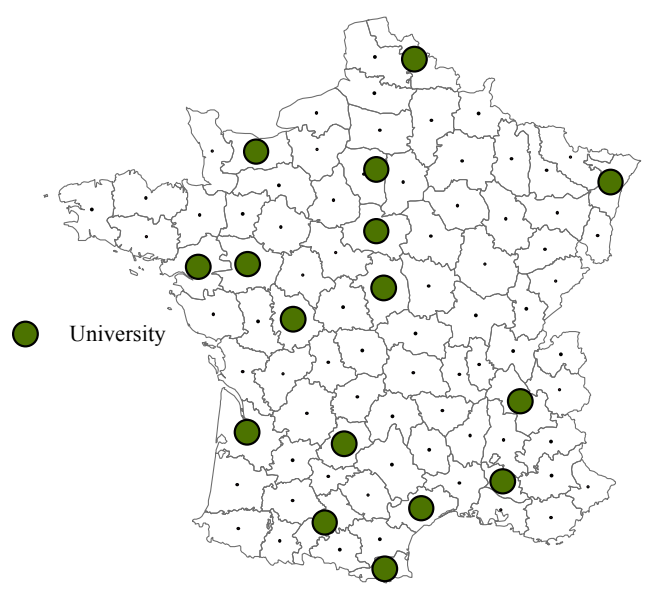

B. Universities in 1700 .

Figure 5: Urban population and universities in 1700

\section{Empirical Methodology}

\subsection{Empirical strategy}

The observed relationship between industrialization and human capital is not necessarily a causal one. It may reflect the impact of economic development on the process of industrialization as well as the influence of institutional, geographical, cultural and human capital characteristics on the joint process of industrialization and human capital accumulation. In light of the endogeneity of industrialization and human capital formation, this research exploits exogenous regional variations in the adoption of the steam engine across France to establish the causal effect of industrialization on human capital.

The identification strategy has two components. The first component is motivated by the historical account of the gradual regional diffusion of the steam engine in France during the $18^{\text {th }}$ and $19^{\text {th }}$ century (Ballot, 1923; Sée, 1925; Léon, 1976). ${ }^{16}$ Considering the positive association between industrialization and the use of the steam engine (Mokyr, 1990; Bresnahan and Trajtenberg, 1995; Rosenberg and Trajtenberg, 2004), the study takes advantage of the regional diffusion of the steam engine to identify the effect of local variations in the intensity of the use of the steam engine during the 1839-1847 period on the process of development. In particular, it exploits the distances between each French department and Fresnes-sur-Escaut (in the Nord department), where the first successful commercial application of the steam engine in France was made in 1732, as an instrument

period, observations are missing for Bas-Rhin, Dordogne, Haut-Rhin, Lot, Morbihan, Seine and Vendée.

${ }^{16}$ There was also a regional pattern in the diffusion of steam engines in England (Kanefsky and Robey, 1980; Nuvolari et al., 2011). 
for the use of the steam engines in $1839-1847 .{ }^{17}$

Consistent with the diffusion hypothesis, the second steam engine in France that was successfully utilized for commercial purposes was operated in 1737 in the mines of Anzin, also in the Nord department, less than $10 \mathrm{~km}$ away from Fresnes-sur-Escaut. Furthermore, in the subsequent decades until the 1789 French Revolution the commercial use of the steam engine expanded predominantly to the nearby northern and north-western regions. Nevertheless, at the onset of the French revolution in 1789, steam engines were less widespread in France than in England. A few additional steam engines were introduced until the fall of the Napoleonic Empire in 1815, notably in Saint-Quentin in 1803 and in Mulhouse in 1812, but it is only after 1815 that the diffusion of steam engines in France accelerated (Sée, 1925; Léon, 1976).

Nevertheless, in light of the confounding effects of geographic, institutional and demographic characteristics on the pace of technological diffusion, as well as on the process of development, in order to mitigate the potential effect of unobserved heterogeneity, the econometric model accounts for a wide range of these characteristics (altitude, latitude, rainfall, land suitability, maritime and border departments, Paris and its suburbs, the distance to Paris).

Indeed, in line with the historical account, the distribution of steam engines across French departments, as reported in the 1839-1847 industrial survey, is indicative of a local diffusion process from Fresnes-sur-Escaut. As reported in Column 1 of Table 1 and shown in Panel A of Figure 6, there is a highly significant negative correlation between the distance from Fresnes-sur-Escaut to the administrative center of each department and the number of steam engines in the department. But as discussed in Section 2.3, pre-industrial development and a wide range of confounding geographical and institutional characteristics could have contributed to the adoption of the steam engine. Reassuringly, the unconditional negative relationship between the distance to Fresnes-surEscaut and the number of steam engines remains highly significant and is larger in absolute value when exogenous confounding geographical controls, i.e., land suitability, latitude, rainfall and temperature (Column 2), as well as institutional factors and pre-industrial development (Column 3), are accounted for. Importantly, the diffusion pattern of steam engines is not significantly correlated with the distance between Paris and the administrative center of each department when the distance from Fresnes-sur-Escaut to each department's administrative center is excluded from the analysis (Column 4). Moreover, Column 5 of Table 1 and Panel B of Figure 6 indicate that there is still a highly significant negative correlation between the distance from Fresnes-sur-Escaut to the administrative center of each department and the intensity of the use of steam engines in the department when the distance to Paris is and the Paris and suburbs dummy variable are included. Specifically, a 100-km increase in the distance from Fresnes-sur-Escaut is associated with

\footnotetext{
${ }^{17}$ This steam engine was used to pump water in an ordinary mine of Fresnes-sur-Escaut. It is unclear whether Pierre Mathieu, the owner of the mine, built the engine himself after a trip in England or employed an Englishman for this purpose (Ballot, 1923, p.385).
} 
Table 1: The geographical diffusion of the steam engine

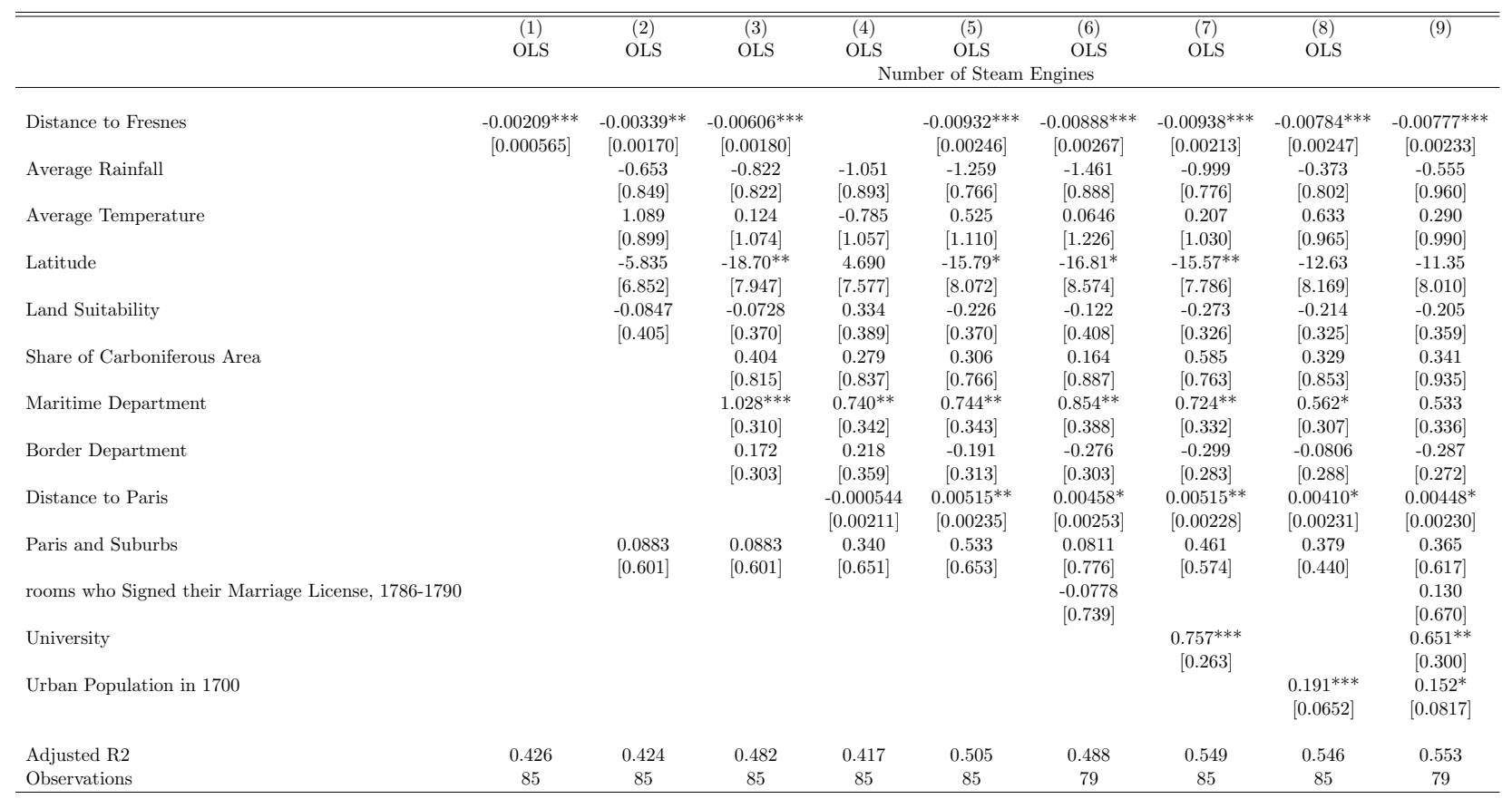

Note: The dependent variable and the explanatory variables except the dummies are in logarithm. The aerial distances are measured in kilometers.

Robust standard errors are reported in brackets. *** indicates significance at the $1 \%$-level, ** indicates significance at the $5 \%$-level, $*$ indicates significance at the $10 \%$-level.

a 0.107 point decrease in the log of the number of steam engines in a given department, relative to the log of the average number of steam engines per department which is equal to 1.47. This means that, for two departments located at the 25 th percentile $(326.69 \mathrm{~km})$ and 75 th percentile $(658.56$ $\mathrm{km}$ ) away from Fresnes, the $331.87 \mathrm{~km}$ difference leads to a 3.55 point decrease in the log of the number of steam engines (i.e., a decrease of 16 engines, relative to a sample mean of 29.2 and a standard deviation of 66.1 ).

Finally, Moreover, the findings suggest that pre-industrial economic and human development, which is captured by the degree of urbanization in each department in 1700, share of grooms who could sign their marriage license in 1786-1790, and the number of universities in 1700 had a persistent positive and significant association with the adoption of the steam engine (Columns (6)-(9) of Table 1 and Panel C of Figure 6). ${ }^{18}$ Nevertheless these pre-industrial characteristics have no qualitative impact on the negative association between the distance from Fresnes-sur-Escaut and the number of steam engines.

Moreover, the highly significant negative correlation between the number of steam engines in each department and the distance from Fresnes-sur-Escaut to the administrative center of each

\footnotetext{
${ }^{18}$ As established in Table B.1, the qualitative results are wnaffect if the share of groms in the $1686-1690$ - Or 1816-1820 period is used instead.
} 


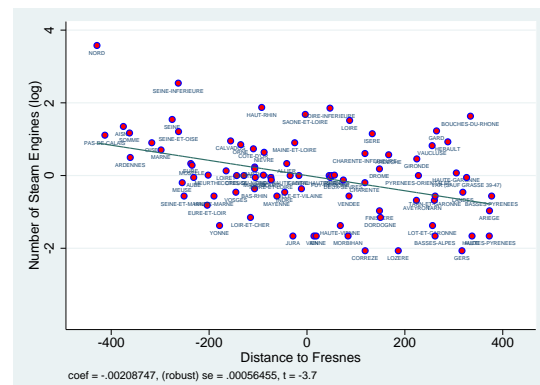

A. Unconditional.

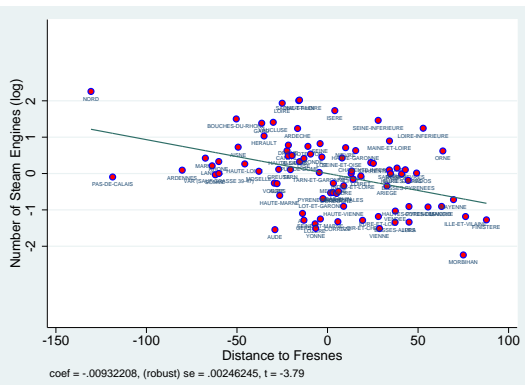

B. Conditional on geography and institutions.

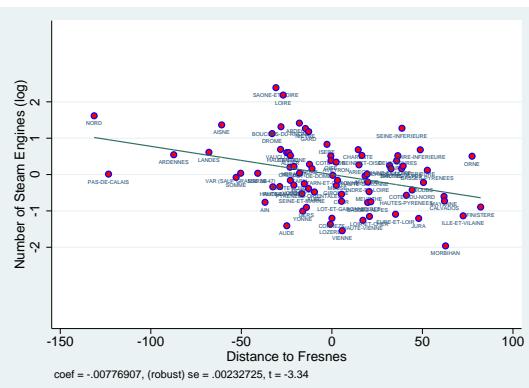

C. Conditional on all controls.

Figure 6: The effect of the distance from Fresnes-sur-Escaut on the number of steam engines in 1839-1847 Note: These figures depict the partial regression line for the effect of the distance from Fresnes-sur-Escaut on the number of steam engines in each French department in 1839-1847. Panel A presents the unconditional relationship while Panel B reports the relationship which controls for geographic and institutional characteristics, as well as for pre-industrial development. Thus, the $\mathrm{x}$ - and $\mathrm{y}$-axes in Panels A, B and C plot the residuals obtained from regressing the number of steam engines and the distance from Fresnes-sur-Escaut, respectively with and without the aforementioned set of covariates.

department is robust to the inclusion of an additional set of confounding geographical, demographic and institutional characteristies, as well as to the forces of pre-industrial development, which as discussed in section 6, may have contributed to the relationship between industrialization and economic development. As established in Table B.1 in the Appendix, these confounding factors, which could be largely viewed as endogenous to the adoption of the steam engine and are thus not part of the baseline analysis, do not affect the qualitative results.

Table 2: The determinants of the diffusion of the steam engine: the insignificance of distances from other major cities

\begin{tabular}{|c|c|c|c|c|c|c|}
\hline & $\begin{array}{l}(1) \\
\text { OLS }\end{array}$ & $\begin{array}{c}(2) \\
\text { OLS }\end{array}$ & $\begin{array}{c}\text { OLS } \\
\text { OLmber of Ste } \\
\text { Number }\end{array}$ & $\begin{array}{c}\text { (4) } \\
\text { OLS } \\
\text { eam Engines }\end{array}$ & $\begin{array}{l}(5) \\
\text { OLS }\end{array}$ & $\begin{array}{c}(6) \\
\text { OLS }\end{array}$ \\
\hline Distance to Fresnes & $\begin{array}{c}-0.0027^{* * *} \\
{[0.00060]}\end{array}$ & $\begin{array}{c}-0.0033^{* * *} \\
{[0.00074]}\end{array}$ & $\begin{array}{c}-0.0027^{* * *} \\
{[0.00058]}\end{array}$ & $\begin{array}{c}-0.0037^{* * *} \\
{[0.0012]}\end{array}$ & $\begin{array}{c}-0.0027^{* * *} \\
{[0.00081]}\end{array}$ & $\begin{array}{c}-0.0020^{* *} \\
{[0.00087]}\end{array}$ \\
\hline Distance to Marseille & & $\begin{array}{l}-0.00077 \\
{[0.00096]}\end{array}$ & & & & \\
\hline Distance to Lyon & & & $\begin{array}{c}0.00016 \\
{[0.00099]}\end{array}$ & & & \\
\hline Distance to Rouen & & & & $\begin{array}{c}0.00115 \\
{[0.00142]}\end{array}$ & & \\
\hline Distance to Mulhouse & & & & & $\begin{array}{l}-0.00012 \\
{[0.00084]}\end{array}$ & \\
\hline Distance to Bordeaux & & & & & & $\begin{array}{c}0.00150 \\
{[0.00106]}\end{array}$ \\
\hline Adjusted $\mathrm{R}^{2}$ & 0.188 & 0.186 & 0.178 & 0.184 & 0.178 & 0.201 \\
\hline Observations & 85 & 85 & 85 & 85 & 85 & 85 \\
\hline
\end{tabular}

Note: The dependent variable is in logarithm. The aerial distances are measured in kilometers. Robust standard errors are reported in brackets. *** indicates significance at the $1 \%$-level, ** indicates significance at the $5 \%$-level, * indicates significance at the $10 \%$-level.

The validity of the aerial distance from Fresnes-sur-Escaut as an instrumental variable for the number of steam engines across France is enhanced by two supplementary robustness checks. First, 
Table 3: The determinants of the diffusion of the steam engine: the insignificance of distances from other major French cities measured in travel weeks

\begin{tabular}{|c|c|c|c|c|c|c|c|}
\hline & $\begin{array}{l}(1) \\
\text { OLS }\end{array}$ & $\begin{array}{c}(2) \\
\text { OLS }\end{array}$ & $\begin{array}{l}(3) \\
\text { OLS }\end{array}$ & $\begin{array}{c}(4) \\
\text { OLS }\end{array}$ & $\begin{array}{c}(5) \\
\text { OLS }\end{array}$ & $\begin{array}{c}(6) \\
\text { OLS }\end{array}$ & $\begin{array}{l}(7) \\
\text { OLS }\end{array}$ \\
\hline & \multicolumn{7}{|c|}{ Number of Steam Engines } \\
\hline Distance from Fresnes (weeks of travel) & $\begin{array}{c}-2.858^{* * * *} \\
{[0.680]}\end{array}$ & $\begin{array}{c}-3.828^{* * *} \\
{[1.117]}\end{array}$ & $\begin{array}{c}-2.772^{* * * *} \\
{[0.661]}\end{array}$ & $\begin{array}{c}-3.077^{* * * *} \\
0.756\end{array}$ & $\begin{array}{c}-2.834^{* * * *} \\
0.741]\end{array}$ & $\begin{array}{c}-2.944^{* * *} \\
{[0.823]}\end{array}$ & $\begin{array}{c}-2.655^{* * *} \\
{[0.844]}\end{array}$ \\
\hline Distance from Paris (weeks of travel) & & $\begin{array}{c}1.314 \\
{[1.242]}\end{array}$ & & & & & \\
\hline Distance from Marseille (weeks of travel) & & & $\begin{array}{c}0.489 \\
{[0.686]}\end{array}$ & & & & \\
\hline Distance from Lyon (weeks of travel) & & & & $\begin{array}{c}0.558 \\
{[0.700]}\end{array}$ & & & \\
\hline Distance from Rouen (weeks of travel) & & & & & $\begin{array}{c}-0.0485 \\
{[0.738]}\end{array}$ & & \\
\hline Distance from Mulhouse (weeks of travel) & & & & & & $\begin{array}{l}0.129 \\
{[0.531]}\end{array}$ & \\
\hline Distance from Bordeaux (weeks of travel) & & & & & & & $\begin{array}{c}0.302 \\
{[0.577]}\end{array}$ \\
\hline Adjusted $\mathrm{R}^{2}$ & 0.147 & 0.146 & 0.144 & 0.145 & 0.137 & 0.137 & 0.139 \\
\hline Observations & 85 & 85 & 85 & 85 & 85 & 85 & 85 \\
\hline
\end{tabular}

Note: The dependent variable is in logarithm. Robust standard errors are reported in brackets. *** indicates significance at the $1 \%$-level, ** indicates significance at the $5 \%$-level, * indicates significance at the $10 \%$-level.

Table 4: Pre-industrial development and the distance from Fresnes-sur-Escaut

\begin{tabular}{lccc}
\hline \hline & $(1)$ & $(2)$ & $(3)$ \\
& Tobit & OLS & Probit \\
& Urban Population in 1700 & Literacy in 1686-1690 & University in 1700 \\
\hline & & & \\
Fresnes sur Escaut & -0.0025 & -0.022 & 0.0012 \\
Average Rainfall & {$[0.0051]$} & {$[0.023]$} & {$[0.0028]$} \\
& $-7.335^{* * *}$ & -11.07 & -1.915 \\
Average Temperature & {$[2.449]$} & {$[10.73]$} & {$[1.170]$} \\
& 2.414 & $-44.74^{* *}$ & 0.368 \\
Latitude & {$[3.475]$} & {$[18.58]$} & {$[2.014]$} \\
& 0.827 & $13.37^{* *}$ & 0.785 \\
Land Suitability & {$[1.500]$} & {$[5.738]$} & {$[0.789]$} \\
& -7.015 & -1.118 & 1.015 \\
$\sigma$ & {$[21.82]$} & {$[85.55]$} & {$[11.71]$} \\
& & & \\
Pseudo R & & & \\
$\mathrm{R}^{2}$ & $2.529^{* * *}$ & & 0.083 \\
Left-censored observations & {$[0.261]$} & 0.456 & \\
Uncensored observations $^{\text {Observations }}$ & 0.081 & & 85 \\
\hline
\end{tabular}

Note: The explanatory variables except the dummies are in logarithm. The aerial distance is measured in kilometers. Literacy in $1786-1790$ is captured by the share of grooms who signed their marriage license during that period. Robust standard errors are reported in brackets. *** indicates significance at the $1 \%$-level, $* *$ indicates significance at the $5 \%$-level, $*$ indicates significance at the $10 \%$-level. 
in Table 2, distances between each department and major centers of economic power in 1839-1847 are shown to be uncorrelated with the number of steam engines over this period. Specifically conditional on the distance from Fresnes-sur-Escaut, distances between each department and Marseille and Lyon (the second and third largest cities in France), Rouen (a major harbor in the north-west where the steam engine was introduced in 1796), Mulhouse (a major city in the east where the steam engine was introduced in 1812), and Bordeaux (a major harbor in the south-west) are uncorrelated with steam engines in 1839-1847, lending credence to the unique role of Fresnes-sur-Escaut and the introduction of the first steam engine in this location in the diffusion of the steam engine across France. Moreover, the use of an alternative measure of distances based on the time needed for a surface travel between any pair of locations (Özak, 2013) in Table 3 does not affect the qualitative results.

Second, the distance from Fresnes-sur-Escaut is uncorrelated with economic development across France in the pre-industrial period. Unlike the highly significant negative relationship between the distance from Fresnes-sur-Escaut and the number of steam engines in 1839-1847, Figures 1 and 5 and Table 4 show that the distance from Fresnes-sur-Escaut was uncorrelated with urban development and human capital formation in the pre-industrial era. Specifically, in Column 1 of Table 4, urbanization rates in 1700 are shown to be uncorrelated with the distance from Fresnessur-Escaut. In Column 2, it appears that literacy rates in the pre-industrial period, as proxied by the share of grooms who signed their marriage license in 1686-1690, are not correlated with the distance from Fresnes-sur-Escaut. Finally, in Column 3, no significant relationship is found between the presence of a university in 1700 and the distance from Fresnes-sur-Escaut. ${ }^{19}$

The second component of the identification strategy is motivated by the general observation that the availability of a technology is not necessarily sufficient for individuals to adopt it. It is however likely that an exogenous income shock would lead to the adoption of an available technology. Namely we consider that a negative (respectively, positive) deviation from local wheat prices would be more (less) conducive to the shift from agriculture to industry in the years just before 1839, when the survey began. For this purpose, we use the data collected by Labrousse et al. (1970) to define the following standardized measure of deviation from average wheat prices over the 1835-1838 period which highlights positive deviations from regular wheat prices

$$
\text { WheatPriceDeviation }_{d, 1834-1838}=\frac{\overline{\text { Price }}_{d, 1834-1838}-\overline{\text { Price }}_{d, 1810-1829}}{\sigma_{d, 1810-1829}}
$$

where $\overline{\text { Price }}_{d, 1834-1838}$ is the average wheat price in department $\boldsymbol{d}$ over the 1834-1838

\footnotetext{
${ }^{19}$ It is worth pointing out that these pre-industrial measures of development are highly correlated with income per-capita in the pest-industrialized period. For example, the urban population in 1700 is positively correlated with all our measures of GDP per capita in 1872 (0.451), 1901 (0.293), $1930(0.551)$ and 2001-2010 (0.517).
} 
Table 5: The determinants of the adoption of the steam engine in 1839-1847: deviations from standard wheat prices in 1834-1838

\begin{tabular}{|c|c|c|c|c|c|c|c|}
\hline & $\begin{array}{c}(1) \\
\text { OLS }\end{array}$ & $\begin{array}{c}(2) \\
\text { OLS }\end{array}$ & $\begin{array}{c}\text { OLS } \\
\text { OL }\end{array}$ & $\begin{array}{c}(4) \\
\text { OLS }\end{array}$ & $\begin{array}{c}(5) \\
\text { OLS }\end{array}$ & $\begin{array}{c}(6) \\
\text { OLS }\end{array}$ & $\begin{array}{c}(7) \\
\text { OLS }\end{array}$ \\
\hline & \multicolumn{7}{|c|}{ Number of Steam Engines } \\
\hline Deviation from Wheat Prices in 1835-1838 (baseline 1810-1829) & $\begin{array}{c}-1.528^{* *} \\
{[0.594]}\end{array}$ & & & & & & \\
\hline Deviation from Wheat Prices in 1835-1838 (baseline 1820-1829) & & $\begin{array}{c}-1.072^{* * *} \\
{[0.234]}\end{array}$ & & & & & \\
\hline Deviation from Wheat Prices in 1835-1838 (baseline 1815-1834) & & & $\begin{array}{c}-1.674^{* * *} \\
{[0.459]}\end{array}$ & & & & \\
\hline Deviation from Wheat Prices in 1835-1838 (baseline 1825-1834) & & & & $\begin{array}{c}-0.957^{* * *} \\
{[0.253]}\end{array}$ & & & \\
\hline Deviation from Wheat Prices in 1831-1834 (baseline 1810-1829) & & & & & $\begin{array}{c}0.108 \\
{[0.685]}\end{array}$ & & \\
\hline Deviation from Wheat Prices in 1839-1842 (baseline 1810-1829) & & & & & & $\begin{array}{c}0.150 \\
{[0.186]}\end{array}$ & \\
\hline Deviation from Wheat Prices in 1843-1846 (baseline 1810-1829) & & & & & & & $\begin{array}{c}-0.687 \\
{[0.492]}\end{array}$ \\
\hline Observations & 85 & 85 & 85 & 85 & 85 & 85 & 85 \\
\hline Adjusted R2 & 0.059 & 0.156 & 0.101 & 0.104 & -0.012 & -0.005 & 0.007 \\
\hline
\end{tabular}

Note: The dependent variable is in logarithm. The aerial distances are measured in kilometers. Robust standard errors are reported in brackets.

*** indicates significance at the $1 \%$-level, ** indicates significance at the $5 \%$-level, * indicates significance at the $10 \%$-level.

Table 6: Steam engine adoption in 1839-1847: the geographical diffusion of the steam engine and deviations from standard wheat prices in 1834-1838

\begin{tabular}{|c|c|c|c|c|c|c|c|c|c|}
\hline & $\begin{array}{l}(1) \\
\text { OLS }\end{array}$ & $\begin{array}{l}(2) \\
\text { OLS }\end{array}$ & $\begin{array}{l}(3) \\
\text { OLS }\end{array}$ & $\begin{array}{l}\text { (4) } \\
\text { OLS }\end{array}$ & $\begin{array}{l}(5) \\
\text { OLS }\end{array}$ & $\begin{array}{c}(6) \\
\text { OLS }\end{array}$ & $\begin{array}{l}(7) \\
\text { OLS }\end{array}$ & $\begin{array}{l}(8) \\
\text { OLS }\end{array}$ & $\begin{array}{l}\text { (9) } \\
\text { OLS }\end{array}$ \\
\hline & \multicolumn{9}{|c|}{ Number of Steam Engines } \\
\hline Distance to Fresnes & $\begin{array}{c}-0.00164^{* * *} * \\
{[0.000535]}\end{array}$ & $\begin{array}{l}-0.00225 \\
{[0.00184]}\end{array}$ & $\begin{array}{c}-0.00442^{* *} \\
{[0.00184]}\end{array}$ & & $\begin{array}{c}-0.00687^{* * *} \\
{[0.00250]}\end{array}$ & $\begin{array}{c}-0.00682^{* *} \\
{[0.00266]}\end{array}$ & $\begin{array}{c}-0.00702^{* * *} \\
{[0.00211]}\end{array}$ & $\begin{array}{c}-0.00585^{* *} \\
{[0.00245]}\end{array}$ & $\begin{array}{c}-0.00610^{\text {*** }} \\
{[0.00230]}\end{array}$ \\
\hline Deviation from Wheat Prices in 1835-1838 (baseline 1810-1829) & $\begin{array}{c}-1.597^{* * * *} \\
{[0.409]}\end{array}$ & $\begin{array}{c}-1.847^{* * *} \\
{[0.410]}\end{array}$ & $\begin{array}{c}-1.792^{* * *} \\
{[0.474]}\end{array}$ & $\begin{array}{c}-2.075 * * * \\
{[0.456]}\end{array}$ & $\begin{array}{c}-1.633^{* * *} \\
{[0.481]}\end{array}$ & $\begin{array}{c}-1.608^{* * *} \\
{[0.527]}\end{array}$ & $\begin{array}{c}-1.571^{*} * * \\
{[0.493]}\end{array}$ & $\begin{array}{c}-1.463^{* * *} \\
{[0.511]}\end{array}$ & $\begin{array}{c}-1.401^{* *} \\
{[0.557]}\end{array}$ \\
\hline Average Rainfall & & $\begin{array}{l}-0.934 \\
{[0.858]}\end{array}$ & $\begin{array}{l}-1.230 \\
{[0.843]}\end{array}$ & $\begin{array}{l}-1.430 \\
{[0.875]}\end{array}$ & $\begin{array}{c}-1.502^{*} \\
{[0.792]}\end{array}$ & $\begin{array}{c}-1.643^{*} \\
{[0.910]}\end{array}$ & $\begin{array}{l}-1.246 \\
{[0.789]}\end{array}$ & $\begin{array}{l}-0.716 \\
{[0.826]}\end{array}$ & $\begin{array}{l}-0.819 \\
{[0.950]}\end{array}$ \\
\hline Average Temperature & & $\begin{array}{c}1.632 \\
{[0.981]}\end{array}$ & $\begin{array}{c}1.489 \\
{[1.158]}\end{array}$ & $\begin{array}{c}1.085 \\
{[1.073]}\end{array}$ & $\begin{array}{l}1.651 \\
{[1.185]}\end{array}$ & $\begin{array}{c}0.912 \\
{[1.241]}\end{array}$ & $\begin{array}{l}1.305 \\
{[1.149]}\end{array}$ & $\begin{array}{c}1.626 \\
{[1.082]}\end{array}$ & $\begin{array}{l}1.005 \\
{[1.083]}\end{array}$ \\
\hline Latitude & & $\begin{array}{l}-2.047 \\
{[7.402]}\end{array}$ & $\begin{array}{l}-11.60 \\
{[8.017]}\end{array}$ & $\begin{array}{l}4.970 \\
{[6.975]}\end{array}$ & $\begin{array}{l}-10.17 \\
{[8.148]}\end{array}$ & $\begin{array}{l}-12.19 \\
{[8.553]}\end{array}$ & $\begin{array}{l}-10.18 \\
{[7.690]}\end{array}$ & $\begin{array}{l}-8.046 \\
{[8.104]}\end{array}$ & $\begin{array}{l}-7.915 \\
{[7.943]}\end{array}$ \\
\hline Land Suitability & & $\begin{array}{l}-0.352 \\
{[0.352]}\end{array}$ & $\begin{array}{l}-0.512 \\
{[0.395]}\end{array}$ & $\begin{array}{l}-0.305 \\
{[0.384]}\end{array}$ & $\begin{array}{l}-0.581 \\
{[0.397]}\end{array}$ & $\begin{array}{l}-0.447 \\
{[0.417]}\end{array}$ & $\begin{array}{c}-0.613^{*} \\
{[0.355]}\end{array}$ & $\begin{array}{l}-0.534 \\
{[0.358]}\end{array}$ & $\begin{array}{l}-0.482 \\
{[0.372]}\end{array}$ \\
\hline Paris and Suburbs & & & $\begin{array}{l}-0.272 \\
{[0.546]}\end{array}$ & $\begin{array}{l}-0.179 \\
{[0.598]}\end{array}$ & $\begin{array}{l}0.0742 \\
{[0.615]}\end{array}$ & $\begin{array}{l}-0.266 \\
{[0.697]}\end{array}$ & $\begin{array}{l}0.0226 \\
{[0.538]}\end{array}$ & $\begin{array}{c}-0.0102 \\
{[0.439]}\end{array}$ & $\begin{array}{l}0.0333 \\
{[0.581]}\end{array}$ \\
\hline Share of Carboniferous Area & & & $\begin{array}{c}0.159 \\
{[0.686]}\end{array}$ & $\begin{array}{l}0.0413 \\
{[0.666]}\end{array}$ & $\begin{array}{c}0.112 \\
{[0.685]}\end{array}$ & $\begin{array}{c}-0.0534 \\
{[0.762]}\end{array}$ & $\begin{array}{c}0.386 \\
{[0.685]}\end{array}$ & $\begin{array}{c}0.152 \\
{[0.760]}\end{array}$ & $\begin{array}{c}0.140 \\
{[0.808]}\end{array}$ \\
\hline Maritime Department & & & $\begin{array}{c}0.714^{* *} \\
{[0.291]}\end{array}$ & $\begin{array}{c}0.483 \\
{[0.299]}\end{array}$ & $\begin{array}{l}0.541^{*} \\
{[0.314]}\end{array}$ & $\begin{array}{l}0.693^{*} \\
{[0.350]}\end{array}$ & $\begin{array}{l}0.530^{*} \\
{[0.302]}\end{array}$ & $\begin{array}{c}0.405 \\
{[0.288]}\end{array}$ & $\begin{array}{c}0.427 \\
{[0.309]}\end{array}$ \\
\hline Border Department & & & $\begin{array}{l}0.469 \\
{[0.318]}\end{array}$ & $\begin{array}{c}0.561 \\
{[0.366]}\end{array}$ & $\begin{array}{l}0.186 \\
{[0.335]}\end{array}$ & $\begin{array}{c}0.130 \\
{[0.329]}\end{array}$ & $\begin{array}{l}0.0692 \\
{[0.318]}\end{array}$ & $\begin{array}{c}0.242 \\
{[0.303]}\end{array}$ & $\begin{array}{l}0.0635 \\
{[0.293]}\end{array}$ \\
\hline Distance to Paris & & & & $\begin{array}{c}-0.000566 \\
{[0.00194]}\end{array}$ & $\begin{array}{c}0.00364 \\
{[0.00227]}\end{array}$ & $\begin{array}{c}0.00317 \\
{[0.00245]}\end{array}$ & $\begin{array}{l}0.00369^{*} \\
{[0.00221]}\end{array}$ & $\begin{array}{c}0.00289 \\
{[0.00221]}\end{array}$ & $\begin{array}{c}0.00329 \\
{[0.00225]}\end{array}$ \\
\hline Grooms who Signed their Marriage License, 1786-1790 & & & & & & $\begin{array}{l}-0.339 \\
{[0.716]}\end{array}$ & & & $\begin{array}{l}-0.111 \\
{[0.648]}\end{array}$ \\
\hline University & & & & & & & $\begin{array}{c}0.721^{* * *} * \\
{[0.239]}\end{array}$ & & $\begin{array}{c}0.600^{* *} \\
{[0.274]}\end{array}$ \\
\hline Urban Population in 1700 & & & & & & & & $\begin{array}{c}0.164^{* * * *} \\
{[0.0614]}\end{array}$ & $\begin{array}{c}0.133^{*} \\
{[0.0730]}\end{array}$ \\
\hline Adjusted R2 & 0.492 & 0.511 & 0.552 & 0.520 & 0.561 & 0.545 & 0.601 & 0.590 & 0.596 \\
\hline Observations & 85 & 85 & 85 & 85 & 85 & 79 & 85 & 85 & 79 \\
\hline
\end{tabular}




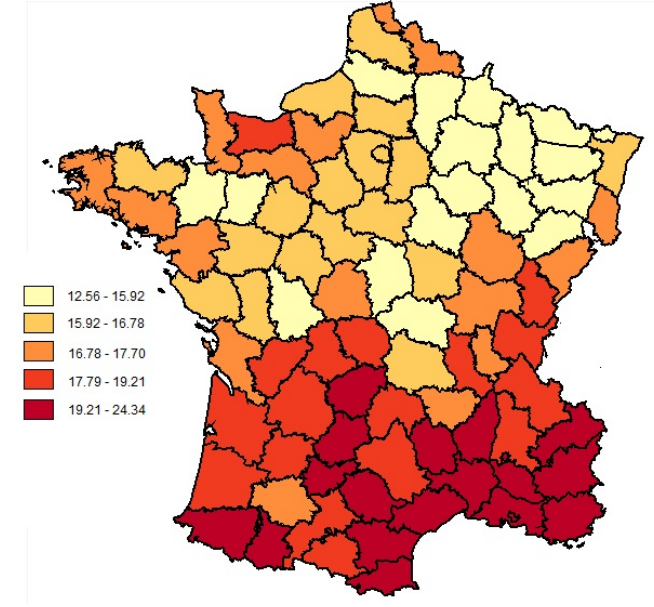

A. Average wheat prices, 1835-1838.

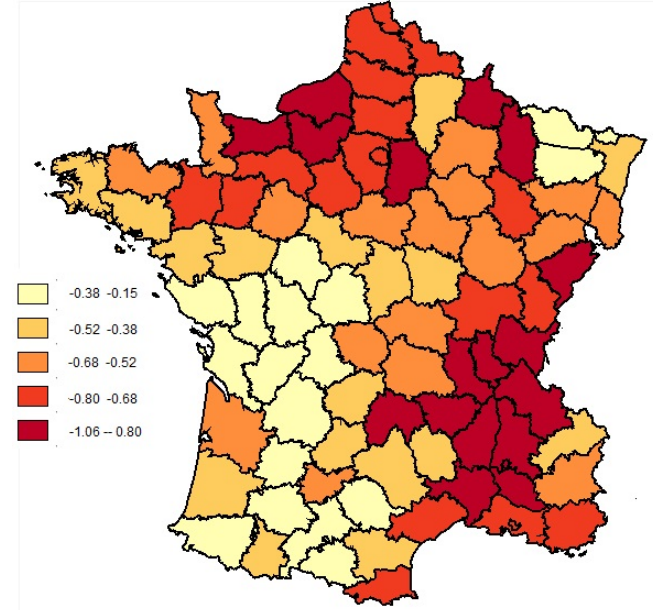

B. Standardized deviation from 1835-1838 wheat prices.

Figure 7: Average wheat prices and Standardized deviation from wheat prices, 1835-1838.

period, $\overline{\text { Price }}_{d, 1810-1829}$ is the average wheat price in department $\boldsymbol{d}$ over our baseline period, i.e., between 1810 and 1829, and and $\sigma_{d, 1810-1829}$ is the standard deviation of wheat price in each season in département $d$ computed over the 1810-1829 baseline period. Panel A of Figure 7 displays the average wheat prices over the 1835-1838 period across the French departments while Panel B of Figure 7 graphs the standardized wheat price deviation in 1835-1838 computed in Equation 1 using the 1820-1820 period as a baseline.

In Column (1) of Table 5, the standardized wheat price deviation defined in Equation 1 is shown to be significantly and negatively correlated with the number of steam engines. This result suggests that areas which experienced high wehat prices were relatively less likely to shift from agriculture to industry and adopt steam engines, as could be expected. The regressions in Columns (2)-(4) show that this effect is robust to using other baseline periods, i.e., 1820-1829, 1815-1834 and 1825-1834. Besides, the validity of this instrument is corroborated by the robustness checks in Columns (5)(8) where we show that price deviations before or after the 1834-1838 period, i.e., in 1831-1834, 1839-1842 and 1843-1846, are not significantly correlated with the adoption of steam engines in 1839-1847.

Table 6 reports the OLS relationship between the number of steam engines and the instrumental variables, the Distance from Fresnes and the Wheat Price Deviation over the 1835-1838 period, progressively adding control variables as in Table $1 .{ }^{20}$ In all

\footnotetext{
${ }^{20}$ In additional specifications, we find that the interaction variable between Distance from Fresnes and the Wheat Price Deviation over the 1835-1838 period is always insignificant.
} 
the specifications, we find that there is a negative and significant correlation between the Distance between Fresnes and the number of steam engines; there is also a negative and significant correlation between a positive wheat price deviation in 1834-1838 and the presence of steam engines in 1839-1847. Specifically, in the regression reported in Column (5) of Table 6 which accounts for geographic and institutional controls, a 100$\mathrm{km}$ increase in the distance from Fresnes-sur-Escaut is associated with a 0.687 point decrease in the log of the number of steam engines in a given department, relative to the log of the average number of steam engines per department which is equal to 1.47. This means that, for two departments located at the 25 th percentile $(326.69 \mathrm{~km})$ and 75 th percentile $(658.56 \mathrm{~km})$ away from Fresnes, the $331.87 \mathrm{~km}$ difference leads to a 2.28 point decrease in the log of the number of steam engines (i.e., a decrease of 9.78 engines, relative to a sample mean of 29.2 and a standard deviation of 66.1). Besides, a one-unit increase in the wheat price deviation leads to a 163.3 point increase in the log of the number of steam engines in a department. As such, for two departments located at the 25th $(-0.764)$ and 75 th $(-0.423)$ percentile of the distribution of wheat price shocks over the 1835-1838 period, this 0.341 difference leads to a 0.557 point decrease in the log of the number of steam engines, i.e., a decrease of 1.75 engines. Hence, these two results suggest that the deviation from standard wheat prices mattered less than the Distance from Fresnes-sur-Escaut. Such findings are in line with the gradual diffusion of steam engines from the North of France as well as with the slow transition of the French regions from agriculture to industry (Cameron and Neal, 2002).

Moreover, the highly significant negative correlation between the number of steam engines in each department, the wheat price deviation over the 1835-1838 period and the distance from Fresnes-sur-Escaut to the administrative center of each department is robust to the inclusion of an additional set of confounding geographical, demographic and institutional characteristics, as well as to the forces of pre-industrial development, which as discussed in section 6 , may have contributed to the relationship between industrialization and economic development. As established in Table B.1 in the Appendix, these confounding factors, which could be largely viewed as endogenous to the adoption of the steam engine and are thus not part of the baseline analysis, do not affect the qualitative results.

\subsection{Empirical Model}

The effect of industrialization on the process of development is estimated using 2SLS. The second stage provides a cross-section estimate of the relationship between the number of steam engines in each department in 1839-1847 to measures of human capital formation at different points in time;

$$
Y_{i t}=\alpha+\beta E_{i}+\boldsymbol{X}_{\boldsymbol{i}}^{\prime} \omega+\varepsilon_{i t},
$$


where $Y_{i t}$ represents a measure of human capital in department $i$ in year $t, E_{i}$ is the $\log$ of the number of steam engines in department $i$ in 1839-1847, $\boldsymbol{X}_{\boldsymbol{i}}^{\prime}$ is a vector of geographical, institutional and pre-industrial economic characteristics of department $i$ and $\epsilon_{i t}$ is an i.i.d. error term for department $i$ in year $t$.

In the first stage, $E_{i}$, the log of the number of steam engines in department $i$ in 1839-1847 is instrumented by $D_{i}$, the aerial distance (in kilometers) between Fresnes-sur-Escaut and the administrative center of department $i$, and $W_{i}$, the wheat price deviation over the 18351838 period in department $i$;

$$
E_{i}=\delta_{1} D_{i}+\delta_{2} W_{i}+\boldsymbol{X}_{\boldsymbol{i}}^{\prime} \delta_{3}+\mu_{i}
$$

where $\boldsymbol{X}_{\boldsymbol{i}}^{\prime}$ is the same vector of geographical, institutional and pre-industrial economic characteristics of department $i$ used in the second stage, and $\mu_{i}$ is an error term for department $i .^{21}$

\section{Industrialization and Human Capital Formation}

The study examines the effect of the number of steam engines in the 1839-1847 period on human capital formation in the short-run. As established in Tables $7-12$, and in line with the proposed hypothesis, the early phase of the industrialization process was conducive to human capital accumulation.

\subsection{The Effect of Industrialization on the Number of Teachers}

The relationship between industrialization and the number of teachers in 1840 and 1863 is presented in Tables 7 and 8. As shown in Column (1), unconditionally, the number of steam engines in industrial production in 1839-1847 had a positive and significant association at the $1 \%$ level with the number of teachers in 1840 and 1863. This relationship remains positive, mostly smaller in magnitude but with the same level of statistical significance, once one progressively accounts for the confounding effects of exogenous geographical factors (Column (2)), institutional factors (Column (3)) and pre-industrial characteristics (Columns (4)-(7)). Finally, mitigating the effect of omitted variables on the observed relationship, the IV estimations in Columns (8)-(12) suggest that the number of steam engines in 1839-1847 had a positive and highly significant impact on the number of teachers in 1840 and 1863, accounting for the confounding effects of geographical, institutional, and demographic characteristics. ${ }^{22}$

\footnotetext{
${ }^{21}$ The aerial distance is a natural proxy for the regional diffusion of the steam engine. In fact, our robustness checks in Section 6 show that accounting for the progressive development of the railroad network in the $19^{\text {th }}$ century not does change our results.

${ }^{22}$ The F-statistic in the first stage is equal to $\mathbf{1 3 . 1 2 6}$ in the absence of pre-industrial controls. Furthermore, the IV coefficient in each specification is larger than the OLS coefficient, which can probably be attributed to measurement
} 
Table 7: The effect of industrialization on the number of teachers in 1840

\begin{tabular}{|c|c|c|c|c|c|c|c|c|c|c|c|c|}
\hline & (1) & $\begin{array}{c}(2) \\
S\end{array}$ & $\begin{array}{c}(3) \\
\text { (3) }\end{array}$ & $\begin{array}{ll}(4) \\
\text { (4) }\end{array}$ & $\begin{array}{ll}(5) \\
S\end{array}$ & $\begin{array}{ll}(6) \\
\text { OIS }\end{array}$ & (7) & (8) & $\begin{array}{l}(9) \\
V\end{array}$ & (10) & (11) & (12) \\
\hline & \multicolumn{12}{|c|}{ Teachers, 1840} \\
\hline Number of Steam Engines & $\begin{array}{c}0.164^{* * * *} \\
{[0.0329]}\end{array}$ & $\begin{array}{c}0.211^{* * * *} \\
{[0.0357]}\end{array}$ & $\begin{array}{c}0.190^{* * *} \\
{[0.0387]}\end{array}$ & $\begin{array}{c}0.193^{* * *} \\
{[0.0402]}\end{array}$ & $\begin{array}{c}0.201^{* * *} * \\
{[0.0380]}\end{array}$ & $\begin{array}{c}0.170^{* * *} \\
{[0.0433]}\end{array}$ & $\begin{array}{c}0.190^{* * *} \\
{[0.0410]}\end{array}$ & $\begin{array}{c}0.334^{* * *} \\
{[0.0912]}\end{array}$ & $\begin{array}{c}0.296^{* * *} \\
{[0.0855]}\end{array}$ & $\begin{array}{c}0.338^{* * *} \\
{[0.08881}\end{array}$ & $\begin{array}{c}0.336^{* * *} \\
{[0.0982]}\end{array}$ & $\begin{array}{c}0.302^{* * *} \\
{[0.0870]}\end{array}$ \\
\hline Average Rainfall & & $\begin{array}{c}1.019^{* * * *} \\
{[0.265]}\end{array}$ & $\begin{array}{c}1.108^{* * *} \\
{[0.283]}\end{array}$ & $\begin{array}{c}0.917^{* * *} \\
{[0.322]}\end{array}$ & $\begin{array}{c}1.086^{* * * *} \\
{[0.286]}\end{array}$ & $\begin{array}{c}1.247^{* * * *} \\
{[0.307]}\end{array}$ & $\begin{array}{c}1.016^{* * * *} \\
{[0.341]}\end{array}$ & $\begin{array}{c}1.259^{* * * *} \\
{[0.281]}\end{array}$ & $\begin{array}{c}1.075^{* * *} \\
{[0.324]}\end{array}$ & $\begin{array}{c}1.195^{* * * *} \\
{[0.279]}\end{array}$ & $\begin{array}{c}1.244^{* * *} \\
{[0.290]}\end{array}$ & $\begin{array}{c}1.060^{* * * *} \\
{[0.323]}\end{array}$ \\
\hline Average Temperature & & $\begin{array}{c}-0.939^{* * *} \\
{[0.269]}\end{array}$ & $\begin{array}{c}-1.146^{* * *} \\
{[0.396]}\end{array}$ & $\begin{array}{l}-0.611 \\
{[0.507]}\end{array}$ & $\begin{array}{c}-1.097^{* * * *} \\
{[0.412]}\end{array}$ & $\begin{array}{c}-1.103^{* * *} \\
{[0.381]}\end{array}$ & $\begin{array}{l}-0.592 \\
{[0.532]}\end{array}$ & $\begin{array}{c}-1.033^{* *} \\
{[0.405]}\end{array}$ & $\begin{array}{l}-0.531 \\
{[0.490]}\end{array}$ & $\begin{array}{c}-0.944^{* *} \\
{[0.430]}\end{array}$ & $\begin{array}{c}-1.038^{* *} \\
{[0.405]}\end{array}$ & $\begin{array}{l}-0.549 \\
{[0.507]}\end{array}$ \\
\hline Latitude & & $\begin{array}{c}1.007 \\
{[1.141]}\end{array}$ & $\begin{array}{c}3.729 \\
{[2.705]}\end{array}$ & $\begin{array}{c}2.813 \\
{[2.852]}\end{array}$ & $\begin{array}{c}3.634 \\
{[2.759]}\end{array}$ & $\begin{array}{c}3.806 \\
{[2.587]}\end{array}$ & $\begin{array}{c}2.716 \\
{[2.855]}\end{array}$ & $\begin{array}{c}3.054 \\
{[2.522]}\end{array}$ & $\begin{array}{c}2.622 \\
{[2.585]}\end{array}$ & $\begin{array}{c}2.941 \\
{[2.603]}\end{array}$ & $\begin{array}{c}3.047 \\
{[2.547]}\end{array}$ & $\begin{array}{c}2.128 \\
{[2.561]}\end{array}$ \\
\hline Land Suitability & & $\begin{array}{c}0.459^{* * *} \\
{[0.157]}\end{array}$ & $\begin{array}{l}0.378^{* *} \\
{[0.159]}\end{array}$ & $\begin{array}{c}0.249 \\
{[0.194]}\end{array}$ & $\begin{array}{l}0.380^{* * *} \\
{[0.161]}\end{array}$ & $\begin{array}{l}0.370^{* * *} \\
{[0.148]}\end{array}$ & $\begin{array}{c}0.258 \\
{[0.191]}\end{array}$ & $\begin{array}{l}0.330^{* * *} \\
{[0.150]}\end{array}$ & $\begin{array}{c}0.222 \\
{[0.177]}\end{array}$ & $\begin{array}{l}0.340^{* *} \\
{[0.151]}\end{array}$ & $\begin{array}{l}0.331^{* *} \\
{[0.151]}\end{array}$ & $\begin{array}{c}0.244 \\
{[0.176]}\end{array}$ \\
\hline Share of Carboniferous Area & & & $\begin{array}{c}-0.635^{* *} \\
{[0.318]}\end{array}$ & $\begin{array}{l}-0.436 \\
{[0.342]}\end{array}$ & $\begin{array}{c}-0.674^{* *} \\
{[0.317]}\end{array}$ & $\begin{array}{c}-0.624^{* *} \\
{[0.311]}\end{array}$ & $\begin{array}{l}-0.483 \\
{[0.332]}\end{array}$ & $\begin{array}{c}-0.675^{* *} \\
{[0.283]}\end{array}$ & $\begin{array}{l}-0.470 \\
{[0.304]}\end{array}$ & $\begin{array}{c}-0.750^{* * *} \\
{[0.281]}\end{array}$ & $\begin{array}{c}-0.676^{* *} \\
{[0.286]}\end{array}$ & $\begin{array}{r}-0.534^{*} \\
{[0.300]}\end{array}$ \\
\hline Maritime Department & & & $\begin{array}{l}0.0926 \\
{[0.127]}\end{array}$ & $\begin{array}{l}0.0478 \\
{[0.149]}\end{array}$ & $\begin{array}{l}0.0873 \\
{[0.125]}\end{array}$ & $\begin{array}{l}0.0732 \\
{[0.129]}\end{array}$ & $\begin{array}{l}0.0364 \\
{[0.162]}\end{array}$ & $\begin{array}{l}-0.0140 \\
{[0.164]}\end{array}$ & $\begin{array}{l}-0.0380 \\
{[0.179]}\end{array}$ & -0.0118 & $\begin{array}{l}-0.0117 \\
{[0.163]}\end{array}$ & $\begin{array}{l}-0.0152 \\
{[0.174]}\end{array}$ \\
\hline Border Department & & & $\begin{array}{c}-0.0604 \\
{[0.120]}\end{array}$ & $\begin{array}{l}-0.146 \\
{[0.135]}\end{array}$ & $\begin{array}{l}-0.0494 \\
{[0.125]}\end{array}$ & $\begin{array}{l}-0.0475 \\
{[0.117]}\end{array}$ & $\begin{array}{l}-0.117 \\
{[0.140]}\end{array}$ & $\begin{array}{l}-0.0917 \\
{[0.121]}\end{array}$ & $\begin{array}{l}-0.150 \\
{[0.121]}\end{array}$ & $\begin{array}{l}-0.0650 \\
{[0.120]}\end{array}$ & $\begin{array}{l}-0.0931 \\
{[0.123]}\end{array}$ & $\begin{array}{l}-0.118 \\
{[0.126]}\end{array}$ \\
\hline Distance to Paris & & & $\begin{array}{c}0.000928 \\
{[0.000834]}\end{array}$ & $\begin{array}{c}0.000909 \\
{[0.000874]}\end{array}$ & $\begin{array}{c}0.000939 \\
{[0.000832]}\end{array}$ & $\begin{array}{c}0.000889 \\
{[0.000814]}\end{array}$ & $\begin{array}{c}0.000859 \\
{[0.000877]}\end{array}$ & $\begin{array}{c}0.00101 \\
{[0.000838]}\end{array}$ & $\begin{array}{c}0.000996 \\
{[0.000838]}\end{array}$ & $\begin{array}{c}0.00102 \\
{[0.000827]}\end{array}$ & $\begin{array}{c}0.00101 \\
{[0.000840]}\end{array}$ & $\begin{array}{c}0.000886 \\
{[0.000822]}\end{array}$ \\
\hline Paris and Suburbs & & & $\begin{array}{c}0.578^{* * *} \\
{[0.195]}\end{array}$ & $\begin{array}{l}0.348^{*} \\
{[0.203]}\end{array}$ & $\begin{array}{c}0.584^{* * * *} \\
{[0.204]}\end{array}$ & $\begin{array}{c}0.562^{* * *} \\
{[0.169]}\end{array}$ & $\begin{array}{l}0.360^{*} \\
{[0.192]}\end{array}$ & $\begin{array}{c}0.529^{* * * *} \\
{[0.145]}\end{array}$ & $\begin{array}{l}0.374^{* *} \\
{[0.151]}\end{array}$ & $\begin{array}{c}0.547^{* * * *} \\
{[0.159]}\end{array}$ & $\begin{array}{c}0.531 * * * \\
{[0.145]}\end{array}$ & $\begin{array}{l}0.345^{* *} \\
{[0.152]}\end{array}$ \\
\hline Grooms who Signed their Marriage License, $1786-1790$ & & & & $\begin{array}{l}0.639^{*} \\
{[0.329]}\end{array}$ & & & $\begin{array}{l}0.577^{*} \\
{[0.338]}\end{array}$ & & $\begin{array}{l}0.589^{*} \\
{[0.312]}\end{array}$ & & & $\begin{array}{l}0.513^{*} \\
{[0.305]}\end{array}$ \\
\hline University & & & & & $\begin{array}{l}-0.0959 \\
{[0.114]}\end{array}$ & & $\begin{array}{l}-0.102 \\
{[0.135]}\end{array}$ & & & $\begin{array}{l}-0.199 \\
{[0.126]}\end{array}$ & & $\begin{array}{l}-0.171 \\
{[0.135]}\end{array}$ \\
\hline Urban Population in 1700 & & & & & & $\begin{array}{c}0.0360 \\
{[0.0331]}\end{array}$ & $\begin{array}{c}0.0272 \\
{[0.0357]}\end{array}$ & & & & $\begin{array}{l}-0.00386 \\
{[0.0291]}\end{array}$ & $\begin{array}{l}0.00453 \\
{[0.0305]}\end{array}$ \\
\hline Adjusted R2 & 0.187 & 0.381 & 0.431 & 0.457 & 0.428 & 0.434 & 0.449 & & & & & \\
\hline Observations & 85 & 85 & 85 & 79 & 85 & 85 & 79 & 85 & 79 & 85 & 85 & 79 \\
\hline & & & & Firs & stage: the & nstrumented & variable is 1 & Number of Ste & am Engines & & & \\
\hline Distance to Fresnes & & & & & & & & $\begin{array}{c}-0.00687^{* * *} \\
{[0.00250]}\end{array}$ & $\begin{array}{c}-0.00682^{* *} \\
{[0.00266]}\end{array}$ & $\begin{array}{c}-0.00702^{* * *} \\
{[0.00211]}\end{array}$ & $\begin{array}{c}-0.00585^{* *} \\
{[0.00245]}\end{array}$ & $\begin{array}{c}-0.00610^{* * * *} \\
{[0.00230]}\end{array}$ \\
\hline $\begin{array}{l}\text { Deviation from Wheat Prices in } 1835-1838 \\
\text { (baseline } 1810-1829 \text { ) }\end{array}$ & & & & & & & & $\begin{array}{c}-1.633^{* * *} \\
{[0.481]}\end{array}$ & $\begin{array}{c}-1.608^{* * *} \\
{[0.527]}\end{array}$ & $\begin{array}{c}-1.571^{* * *} \\
{[0.493]}\end{array}$ & $\begin{array}{c}-1.463^{* * *} \\
{[0.511]}\end{array}$ & $\begin{array}{c}-1.401^{* *} \\
{[0.557]}\end{array}$ \\
\hline F-stat ( $1^{\text {st }}$ stage $)$ & & & & & & & & 13.126 & 10.072 & 14.403 & 8.658 & 8.043 \\
\hline Prob J-Stat & & & & & & & & 0.418 & 0.415 & 0.449 & 0.415 & 0.472 \\
\hline
\end{tabular}

Note: The explanatory variables except the dummies are in logarithm. The aerial distances are measured in kilometers. Robust standard errors are reported in brackets. *** indicates significance at the $1 \%$-level, ** indicates significance at the $5 \%$-level, * indicates significance at the $10 \%$-level.

The regressions in Tables 7 and 8 also account for a large number of confounding geographical and institutional factors, which are discussed above in Section 2.3. First, the climatic and soil characteristics of each department (i.e., land suitability, average temperature, average rainfall, and latitude) could have affected natural land productivity and therefore the feasibility and profitability of the transition to the industrial stage of development, as well as the evolution of income per capita and its potential direct on human capital formation in each department.

Second, the location of departments (i.e., latitude, border departments, maritime departments and departments at a greater distance from the concentration of political power in Paris) could have affected the diffusion of the steam engine and human capital formation. However, most of these factors appear orthogonal to the evolution of human capital, except for average rainfall and the dummy variable for Paris and its suburbs which has have a positive and nearly systematically

error in the explanatory variable - the number of steam engines. Finally, the positive and significant effect of industrialization on the number of teachers in 1840 and 1863 in the IV regressions is corroborated by the reduced form regressions reported in Columns (1)-(2) of Table B.2 in the Appendix, where the instrument Distance to Fresnes is and the Deviation from Wheat Prices in 1835-1838 are found to be negatively and significantly correlated with the number of teachers. 
Table 8: The effect of industrialization on the number of teachers in 1863

\begin{tabular}{|c|c|c|c|c|c|c|c|c|c|c|c|c|}
\hline & $\begin{array}{l}(1) \\
\text { OLS }\end{array}$ & $\begin{array}{c}(2) \\
\text { OLS }\end{array}$ & $\begin{array}{l}(3) \\
\text { OLS }\end{array}$ & $\begin{array}{l}(4) \\
\text { OLS }\end{array}$ & $\begin{array}{l}(5) \\
\text { OLS }\end{array}$ & $\begin{array}{l}6) \\
\text { OLS } \\
\end{array}$ & $\begin{array}{c}\text { (7) } \\
\text { OLS } \\
\text { achers, } 1863\end{array}$ & $\begin{array}{l}\text { (8) } \\
\text { IV }\end{array}$ & $\begin{array}{l}\text { (9) } \\
\text { IV }\end{array}$ & $\begin{array}{l}\text { (10) } \\
\text { IV }\end{array}$ & $\begin{array}{l}\text { (11) } \\
\text { IV }\end{array}$ & $\begin{array}{l}(12) \\
\text { IV }\end{array}$ \\
\hline Number of Steam Engines & $\begin{array}{c}0.198 * * * \\
{[0.0248]}\end{array}$ & $\begin{array}{c}0.214^{* * *} \\
{[0.0283]}\end{array}$ & $\begin{array}{c}0.200 * * * \\
{[0.0283]}\end{array}$ & $\begin{array}{c}0.196 * * * \\
{[0.0277]}\end{array}$ & $\begin{array}{c}0.184^{* * *} \\
{[0.0269]}\end{array}$ & $\begin{array}{l}0.170^{* * * *} \\
{[0.0313]}\end{array}$ & $\begin{array}{c}0.176^{* * *} \\
{[0.0305]}\end{array}$ & $\begin{array}{l}0.283 * * * \\
{[0.0534]}\end{array}$ & $\begin{array}{c}0.289^{* * *} \\
{[0.0562]}\end{array}$ & $\begin{array}{c}0.283 * * * \\
{[0.0540]}\end{array}$ & $\begin{array}{c}0.258^{* * *} \\
{[0.0593]}\end{array}$ & $\begin{array}{c}0.287^{* * *} \\
{[0.0628]}\end{array}$ \\
\hline Average Rainfall & & $\begin{array}{c}0.682^{* * * *} \\
{[0.223]}\end{array}$ & $\begin{array}{c}0.759^{* * * *} \\
{[0.221]}\end{array}$ & $\begin{array}{c}0.678^{* * *} \\
{[0.254]}\end{array}$ & $\begin{array}{c}0.792^{* * *} \\
{[0.217]}\end{array}$ & $\begin{array}{c}0.962^{* * *} \\
{[0.261]}\end{array}$ & $\begin{array}{c}0.797^{* * *} \\
{[0.294]}\end{array}$ & $\begin{array}{c}0.846^{* * *} \\
{[0.224]}\end{array}$ & $\begin{array}{c}0.822^{* * *} \\
{[0.271]}\end{array}$ & $\begin{array}{c}0.870^{* * *} \\
{[0.217]}\end{array}$ & $\begin{array}{c}0.960^{* * * *} \\
{[0.240]}\end{array}$ & $\begin{array}{c}0.840^{* * *} \\
{[0.281]}\end{array}$ \\
\hline Average Temperature & & $\begin{array}{l}-0.0337 \\
{[0.223]}\end{array}$ & $\begin{array}{l}-0.413 \\
{[0.316]}\end{array}$ & $\begin{array}{l}-0.454 \\
{[0.360]}\end{array}$ & $\begin{array}{l}-0.487^{*} \\
{[0.289]}\end{array}$ & $\begin{array}{l}-0.349 \\
{[0.282]}\end{array}$ & $\begin{array}{l}-0.421 \\
{[0.344]}\end{array}$ & $\begin{array}{l}-0.348 \\
{[0.304]}\end{array}$ & $\begin{array}{l}-0.381 \\
{[0.346]}\end{array}$ & $\begin{array}{l}-0.378 \\
{[0.297]}\end{array}$ & $\begin{array}{l}-0.315 \\
{[0.281]}\end{array}$ & $\begin{array}{l}-0.378 \\
{[0.344]}\end{array}$ \\
\hline Latitude & & $\begin{array}{l}1.737^{* *} \\
{[0.801]}\end{array}$ & $\begin{array}{l}4.163^{* *} \\
{[1.843]}\end{array}$ & $\begin{array}{l}3.657^{*} \\
{[1.924]}\end{array}$ & $\begin{array}{l}4.305^{* *} \\
{[1.816]}\end{array}$ & $\begin{array}{l}4.274^{* *} \\
{[1.626]}\end{array}$ & $\begin{array}{l}4.041^{* *} \\
{[1.909]}\end{array}$ & $\begin{array}{l}3.773^{* *} \\
{[1.737]}\end{array}$ & $\begin{array}{l}3.484^{*} \\
{[1.798]}\end{array}$ & $\begin{array}{l}3.806^{* *} \\
{[1.717]}\end{array}$ & $\begin{array}{l}3.872^{* * *} \\
{[1.588]}\end{array}$ & $\begin{array}{l}3.461^{*} \\
{[1.817]}\end{array}$ \\
\hline Land Suitability & & $\begin{array}{c}0.139 \\
{[0.124]}\end{array}$ & $\begin{array}{l}0.211 \\
{[0.137]}\end{array}$ & $\begin{array}{c}0.187 \\
{[0.144]}\end{array}$ & $\begin{array}{l}0.208 \\
{[0.130]}\end{array}$ & $\begin{array}{l}0.199^{*} \\
{[0.112]}\end{array}$ & $\begin{array}{c}0.179 \\
{[0.133]}\end{array}$ & $\begin{array}{c}0.183 \\
{[0.127]}\end{array}$ & $\begin{array}{c}0.162 \\
{[0.138]}\end{array}$ & $\begin{array}{l}0.179 \\
{[0.124]}\end{array}$ & $\begin{array}{c}0.179 \\
{[0.112]}\end{array}$ & $\begin{array}{l}0.165 \\
{[0.137]}\end{array}$ \\
\hline Share of Carboniferous Area & & & $\begin{array}{l}-0.127 \\
{[0.243]}\end{array}$ & $\begin{array}{l}0.0158 \\
{[0.256]}\end{array}$ & $\begin{array}{l}-0.0695 \\
{[0.241]}\end{array}$ & $\begin{array}{l}-0.111 \\
{[0.233]}\end{array}$ & $\begin{array}{l}0.0247 \\
{[0.243]}\end{array}$ & $\begin{array}{l}-0.150 \\
{[0.225]}\end{array}$ & $\begin{array}{c}-0.0149 \\
{[0.236]}\end{array}$ & $\begin{array}{l}-0.124 \\
{[0.223]}\end{array}$ & $\begin{array}{l}-0.139 \\
{[0.213]}\end{array}$ & $\begin{array}{l}-0.0251 \\
{[0.231]}\end{array}$ \\
\hline Maritime Department & & & $\begin{array}{c}0.0686 \\
{[0.0859]}\end{array}$ & $\begin{array}{c}0.102 \\
{[0.0976]}\end{array}$ & $\begin{array}{c}0.0765 \\
{[0.0877]}\end{array}$ & $\begin{array}{c}0.0404 \\
{[0.0825]}\end{array}$ & $\begin{array}{l}0.0741 \\
{[0.103]}\end{array}$ & $\begin{array}{l}0.00714 \\
{[0.101]}\end{array}$ & $\begin{array}{l}0.0240 \\
{[0.116]}\end{array}$ & $\begin{array}{l}0.00537 \\
{[0.104]}\end{array}$ & $\begin{array}{c}-0.00462 \\
{[0.0955]}\end{array}$ & $\begin{array}{l}0.0232 \\
{[0.113]}\end{array}$ \\
\hline Border Department & & & $\begin{array}{l}-0.155 \\
{[0.0936]}\end{array}$ & $\begin{array}{l}-0.153 \\
{[0.105]}\end{array}$ & $\begin{array}{l}-0.171^{*} \\
{[0.0925]}\end{array}$ & $\begin{array}{c}-0.136 \\
{[0.0920]}\end{array}$ & $\begin{array}{l}-0.148 \\
{[0.106]}\end{array}$ & $\begin{array}{l}-0.173^{*} \\
{[0.0907]}\end{array}$ & $\begin{array}{l}-0.157^{*} \\
{[0.0923]}\end{array}$ & $\begin{array}{c}-0.182^{* *} \\
{[0.0901]}\end{array}$ & $\begin{array}{l}-0.160^{*} \\
{[0.0901]}\end{array}$ & $\begin{array}{l}-0.150 \\
{[0.0955]}\end{array}$ \\
\hline Distance to Paris & & & $\begin{array}{l}0.000937^{*} \\
{[0.000556]}\end{array}$ & $\begin{array}{c}0.000856 \\
{[0.000592]}\end{array}$ & $\begin{array}{c}0.000920 \\
{[0.000567]}\end{array}$ & $\begin{array}{c}0.000879 \\
{[0.000537]}\end{array}$ & $\begin{array}{c}0.000895 \\
{[0.000600]}\end{array}$ & $\begin{array}{l}0.000982^{*} \\
{[0.000558]}\end{array}$ & $\begin{array}{c}0.000935 \\
{[0.000587]}\end{array}$ & $\begin{array}{l}0.000978^{*} \\
{[0.000562]}\end{array}$ & $\begin{array}{l}0.000944^{*} \\
{[0.000542]}\end{array}$ & $\begin{array}{c}0.000921 \\
{[0.000586]}\end{array}$ \\
\hline Paris and Suburbs & & & $\begin{array}{l}0.718^{* *} \\
{[0.332]}\end{array}$ & $\begin{array}{c}0.328^{* * *} \\
{[0.0856]}\end{array}$ & $\begin{array}{c}0.710^{* *} \\
{[0.300]}\end{array}$ & $\begin{array}{l}0.695^{* *} \\
{[0.274]}\end{array}$ & $\begin{array}{c}0.368^{* * *} \\
{[0.0994]}\end{array}$ & $\begin{array}{c}0.690^{* *} \\
{[0.285]}\end{array}$ & $\begin{array}{c}0.352^{* * *} \\
{[0.108]}\end{array}$ & $\begin{array}{c}0.683^{* *} \\
{[0.266]}\end{array}$ & $\begin{array}{c}0.678^{* * * *} \\
{[0.254]}\end{array}$ & $\begin{array}{c}0.354^{* * *} \\
{[0.116]}\end{array}$ \\
\hline Grooms who Signed their Marriage License, $1786-1790$ & & & & $\begin{array}{l}0.114 \\
{[0.186]}\end{array}$ & & & $\begin{array}{c}0.118 \\
{[0.189]}\end{array}$ & & $\begin{array}{l}0.0683 \\
{[0.188]}\end{array}$ & & & $\begin{array}{l}0.0550 \\
{[0.190]}\end{array}$ \\
\hline University & & & & & $\begin{array}{c}0.143 \\
{[0.0920]}\end{array}$ & & $\begin{array}{c}0.0445 \\
{[0.0946]}\end{array}$ & & & $\begin{array}{c}0.0696 \\
{[0.0974]}\end{array}$ & & $\begin{array}{l}-0.0237 \\
{[0.104]}\end{array}$ \\
\hline Urban Population in 1700 & & & & & & $\begin{array}{c}0.0524^{* *} \\
{[0.0262]}\end{array}$ & $\begin{array}{c}0.0282 \\
{[0.0262]}\end{array}$ & & & & $\begin{array}{c}0.0313 \\
{[0.0268]}\end{array}$ & $\begin{array}{l}0.00586 \\
{[0.0255]}\end{array}$ \\
\hline Adjusted R2 & 0.411 & 0.464 & 0.539 & 0.540 & 0.550 & 0.568 & 0.539 & & & & & \\
\hline Observations & 85 & 85 & 85 & 79 & 85 & 85 & 79 & 85 & 79 & 85 & 85 & 79 \\
\hline & & & & & stage: the & strumente & variable is & umber of Ste & am Engines & & & \\
\hline $\begin{array}{l}\text { Deviation from Wheat Prices in 1835-1838 } \\
\text { (baseline 1810-1829) }\end{array}$ & & & & & & & & $\begin{array}{c}-0.00687^{* * *} \\
{[0.00250]} \\
-1.633^{* * *} \\
{[0.481]}\end{array}$ & $\begin{array}{c}-0.00682^{* *} \\
{[0.00266]} \\
-1.608^{* * *} \\
{[0.527]}\end{array}$ & $\begin{array}{c}-0.00702^{* * *} \\
{[0.00211]} \\
-1.571^{* * *} \\
{[0.493]}\end{array}$ & $\begin{array}{c}-0.00585^{* *} \\
{[0.00245]} \\
-1.463^{* * *} \\
{[0.511]}\end{array}$ & $\begin{array}{c}-0.00610^{* * * *} \\
{[0.00230]} \\
-1.401^{* *} \\
{[0.557]}\end{array}$ \\
\hline $\begin{array}{l}\text { F-stat ( } 1^{\text {st }} \text { stage) } \\
\text { Prob J-Stat }\end{array}$ & & & & & & & & $\begin{array}{c}13.126 \\
0.361\end{array}$ & $\begin{array}{c}10.072 \\
0.461\end{array}$ & $\begin{array}{c}14.403 \\
0.345\end{array}$ & $\begin{array}{l}8.658 \\
0.388\end{array}$ & $\begin{array}{l}8.043 \\
0.482\end{array}$ \\
\hline
\end{tabular}

Note: The explanatory variables except the dummies are in logarithm. The aerial distances are measured in kilometers. Robust standard errors are reported in brackets. *** indicates significance at the $1 \%$-level, ** indicates significance at the $5 \%$-level, * indicates significance at the $10 \%$-level.

significant correlation with the number of teachers in the OLS and IV regressions in Tables 7 and 8.

Third, the regressions in Tables 7 and 8 take into account the potentially confounding effects of the level of human capital and economic development in the pre-industrial period, as captured by the share of grooms who could sign their marriage license over the 1786-1790 period, the degree of urbanization in each department in 1700 and the number of universities in $1700 .^{23}$ The findings suggest that pre-industrial human capital and development (and the characteristics that may have fostered them) had no significant impact on the number of teachers in 1840 and 1863.

Fourth, the IV estimates in Column (8) of Tables 7 and 8 suggest that the presence of steam engines had large quantitative effects on the number of teachers in primary schools: a one-percent increase in the number of steam engines in a department in 1839-1847 increased the number of teachers in the primary schools by $\mathbf{3 3 . 4 \%}$ in 1840 and $\mathbf{2 8 . 3 \%}$ in 1863 . Hence the 675 percent increase in the number of steam engines in 1839-1847 due to a movement from the 25th percentile

\footnotetext{
${ }^{23}$ As established in Table B.4, the qualitative results are unaffected if the share of grooms in the 1686-1690 or 1816-1820 period is used instead.
} 
(i.e., 4 engines) to the 75 th percentile (i.e., 31 engines) would have led to an increase in the number of teachers of $\mathbf{2 2 5 . 4 5}$ in 1840 and $\mathbf{1 9 1 . 0 3}$ in 1863 (relative to a sample mean of 742 in 1840 and 1243 in 1863).

Finally, the association between the number of steam engines and the number of teachers in 1840 and 1863 is not affected by spatial correlation as established in Tables E.1 and E.2 in the Appendix, accounting for radii of $50 \mathrm{~km}, 100 \mathrm{~km}, 250 \mathrm{~km}$ and $500 \mathrm{~km}$.

\subsection{The Effect of Industrialization on the Share of Pupils in the Population}

\subsubsection{Pupils in Primary Schools}

The effect of industrialization on the number of pupils enrolled in the primary schools of each department (per 10,000 inhabitants) in 1840 and 1863 is reported in Tables 9 and 10. In Table 9 both Tables, the unconditional relationship between the number of steam engines in industrial production in 1839-1847 and the share of pupils in 1840 and in 1863 is positive and significant (Column (1)), and remains so when the confounding effects of exogenous geographical factors (Column (2)) and, institutional factors (Column (3)) and pre-industrial characteristics (Columns (4)(7)) are accounted for. However this positive coefficient becomes insignificant once pre-industrial characteristics (Columns (4)-(7)) are taken into account. In Table 10, the relationship between the number of steam engines in industrial production in 1839-1847 and the share of pupils in 1863 is positive and insignificant when no control variable is included (Column (1)), and is positive and significant once control variables are accounted for (Columns (2)-(7)).

The IV estimates in Columns (8)-(12) in Tables 9 and 10 suggest that the number of steam engines in 1839-1847 had a positive and significant impact on the number of pupils enrolled in the primary schools of each department (per 10,000 inhabitants) in 1840 and 1863, accounting for the confounding effects of geographical, institutional and pre-industrial characteristics. ${ }^{24}$ This positive effect appears to diminish between 1840 and 1863. In addition, in the IV regressions in Tables 9 and 10, there is a strongly positive and significant association between early literacy, as captured by the share of grooms who signed their marriage license in 1786-1790, and the number of pupils. ${ }^{25}$ However, the share of carboniferous area in the department, as well as the presence of a university and the urban population in 1700, are negatively, and in some regressions significantly,

\footnotetext{
${ }^{24}$ The F-statistic in the first stage is equal to 13.126. Furthermore, the IV coefficient in each specification is larger than the OLS coefficient, which can probably be attributed to measurement error in the explanatory variablethe number of steam engines. Finally, the positive and significant effect of industrialization on the number of pupils in 1840 and 1863 in the IV regressions is corroborated by the reduced form regressions reported in Columns (3)-(4) of Table B.2 in the Appendix, where the instrument Distance to Fresnes is found to be negatively and significantly correlated with the number of pupils in 1840 and in 1863 and where the instrument the Deviation from Wheat Prices has a negative impact on the number of pupils in 1840 and 1863 which is only significant in 1840.

${ }^{25}$ As established in Table B.4, the qualitative results are not affected if the share of grooms in the 1686-1690 or 1816-1820 period is used instead.
} 
correlated with the enrollment of pupils. The other characteristics of departments, i.e., latitude, land suitability, border departments, maritime departments as well as the distance to Paris, are shown to have no effect on the enrollment of pupils in the IV regressions. Overall, the IV estimates in Column (8) of Tables 9 and 10 suggest that a one-percent increase in the number of steam engines in a department in 1839-1847 increased the number of pupils enrolled in the primary schools (per 10,000 inhabitants) by $\mathbf{2 9 . 4 \%}$ in 1840 and $\mathbf{1 1 . 7 \%}$ in $\mathbf{1 8 6 3}$. As such, if a department had increased its number of steam engines in 1839-1847 from the 25 th percentile (i.e., 4 engines) to the 75 th percentile (i.e., 31 engines), this 675 percent increase in the number of steam engines would have experienced an increase in the number of pupils of $\mathbf{1 9 6 . 4 3}$ in 1840 and $\mathbf{7 8 . 9 8}$ in 1863 (relative to sample mean of 874 in 1840 and 1179 in 1863, per 10,000 inhabitants).

Finally, the association between intensity of the steam engines and the number of pupils enrolled in the primary schools of each department (per 10,000 inhabitants) in 1840 and 1863 is not affected by spatial correlation, when we account for radii of $50 \mathrm{~km}, 100 \mathrm{~km}, 250 \mathrm{~km}$ and $500 \mathrm{~km}$ as shown in Tables E.3 and E.4 in the Appendix.

\subsubsection{Pupils in Technical Schools}

The effect of industrialization on the number of apprentices in each department (per 10,000 inhabitants) in 1863 is reported in Table 11. The unconditional relationship between the number of steam engines in industrial production in 1839-1847 and the share of apprentices in 1863 is positive and significant (Column (1)), and remains so when the confounding effects of exogenous geographical factors (Column (2)), institutional factors (Column (3)) and pre-industrial characteristics (Columns (4)-(7)) are accounted for.

The IV estimates in Columns (8)-(12) in Table 11 suggest that the number of steam engines in 1839-1847 had a positive and significant impact on the number of apprentices in each department (per 10,000 inhabitants) in 1863, accounting for the confounding effects of geographical, institutional and pre-industrial characteristics. ${ }^{26}$ Overall, the IV estimates in Column (8) of Table 11 suggest that a one-percent increase in the number of steam engines in a department in 1839-1847 increased the number of apprentices (per 10,000 inhabitants) by $\mathbf{6 0 . 3 \%}$ in 1863 . As such, if a department had increased its number of steam engines in 1839-1847 from the 25 th percentile (i.e., 4 engines) to the 75 th percentile (i.e., 31 engines), this 675 percent increase in the number of steam engines would have led to an increase of 407.3 in the number of apprentices per 10,000 inhabitants in 1863 (relative to sample mean of 2.71 in 1863 and a standard deviation of $\mathbf{5 . 9 8}$, per 10,000

\footnotetext{
${ }^{26}$ The F-statistic in the first stage is equal to 13.126. Furthermore, he IV coefficient in each specification is larger than the OLS coefficient, which can probably be attributed to measurement error in the explanatory variable - the number of steam engines. Finally, the positive and significant effect of industrialization on the number of apprentices in 1863 in the IV regressions is corroborated by the reduced form regressions reperted in Column (5) of Table B.2 in the Appendix, where the instrument Distance to Fresnes is found to be negatively and significantly correlated with the number of apprentices.
} 
inhabitants). Arguably, this is a large effect given the actual number of apprentices and suggests that technical education should have been more developed in France than it actually was, in line with anecdotal evidence about the relative lack of interest for technical education in 19th century France which we discussed in Section 2.1.2.

Finally, spatial correlation does not affect the association between the number of steam engines and the number of apprentices in 1863 as established in Table E.5 in the Appendix when one accounts for radii of $50 \mathrm{~km}, 100 \mathrm{~km}, 250 \mathrm{~km}$ and $500 \mathrm{~km}$.

\subsection{The Effect of Industrialization on Literacy}

The relationship between industrialization and literacy, as captured by the share of army conscripts who could read and write over the 1859-1868 period, is presented in Table $12 .{ }^{27}$ In Column (1), unconditionally, the number of steam engines in 1839-1847 had a significant positive association with the share of literate conscripts. However, in the OLS regressions in Columns (2)-(7), this positive association is not significant once the confounding effects of exogenous geographical, institutional and pre-industrial factors are taken into account. Nevertheless, in the IV regressions in Columns (8)-(12) which mitigate the effect of omitted variables on the observed relationship and account for the confounding effects of geographical, institutional and pre-industrial characteristics, the effect of industrialization on the share of literate conscripts is found to be positive and significant.

Moreover, the regressions in Table 12 account for the impact of confounding geographical and institutional factors. In some of the IV regressions in Columns (8)-(12) of Table 12, land suitability had a significant positive association with the share of literate conscripts while temperature had a significant negative relationship, but the coefficients are only significant when the share of grooms who could sign their marriage license in 1786-1790 is excluded from the regressions. ${ }^{28}$ In addition, the variables which control for the location of departments (i.e., latitude, border departments, maritime departments, departments at a greater distance from the concentration of political power in Paris) and pre-industrial development (the number of universities and urban population in 1700) appear orthogonal to industrialization and literacy. As could be expected, the dummy variable for Paris and its suburbs, as well as the share of grooms who could sign their marriage license in 17861790, are significantly and positively associated with the share of literate conscripts in 1859-1868. All in all, the IV estimate in Column (8) of Table 12 indicates that a one-percent increase in the number of steam engines in a department in 1839-1847 increased the share of literate conscripts

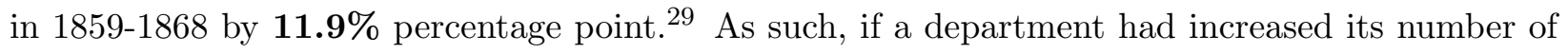

\footnotetext{
${ }^{27}$ Regression results using the share of literate conscripts over the 1847-1856 decade are similar to those obtained over the 1859-1868 period and are reported in Table C.1 in the Appendix.

${ }^{28}$ As shown in Table B.4, the qualitative results are not affected if the share of grooms in the 1686-1690 or 1816-1820 period is used instead.

${ }^{29}$ The F-statistic in the first stage is equal to 13.126. Moreover, the IV coefficient in each specification is larger than the OLS coefficient, which can probably be attributed to measurement error in the independent variable - the number
} 
steam engines in 1839-1847 from the 25 th percentile (i.e., 4 engines) to the 75 th percentile (i.e., 31 engines), this 675 percent increase in the number of steam engines would have led to a $\mathbf{8 0 . 3 3 \%}$ increase in the share of literate conscripts in 1859-1868 (relative to sample mean of 73.57 per cent and a standard deviation of 14.96).

Finally, as we establish in Table E.6 in the Appendix, the association between the number of steam engines and the share of literate conscripts is not affected by spatial correlation for radii of $50 \mathrm{~km}, 100 \mathrm{~km}, 250 \mathrm{~km}$ and $500 \mathrm{~km}$.

\section{Robustness Analysis}

\subsection{Confounding Factors}

This subsection examines the robustness of the baseline analysis to the inclusion of additional confounding geographical, demographic, institutional and pre-industrial characteristics, which may have contributed to the relationship between industrialization and economic development. These confounding factors could be viewed as endogenous to the adoption of the steam engine and are thus not part of the baseline analysis. ${ }^{30}$

In what follows, the analysis focuses on the potential impact of these confounding factors on the baseline IV regressions in Tables 7 to 12, where the dependent variables are the number of teachers in 1840 and 1863, the share of pupils in the population in 1840 and 1863, the share of apprentices in the population in 1863 and the share of literate conscripts over the 1859-1868 period.

\subsubsection{Distance from London}

Given the early industrial use of the steam engine in England and the intensive trade relationship between France and England, human capital in French departments could have been affected by their proximity to England (proxied by the aerial distance between London and the administrative center of each department), which would be correlated with the diffusion of the steam engine in France and with the process of human and economic development. As reported in Table B.3 in Appendix B, accounting for the aerial distance from England has no qualitative impact on the estimated effect of industrialization on human capital.

of steam engines. Finally, the positive and significant effect of industrialization on the share of literate conscripts in 1859-1868 in the IV regressions is corroborated by the reduced form regressions reported in Column (6) of Table B.2 in the Appendix, where the instrument Distance to Fresnes is found to be negatively and significantly correlated with the share of literate conscripts while the Wheat Price Deviation in 1835-1838 is also negatively correlated with the share of literate conscripts and nearly significant with a p-value of 0.101 .

${ }^{30}$ Some of these characteristics could be viewed as "bad controls" (Angrist and Pischke, 2009). 


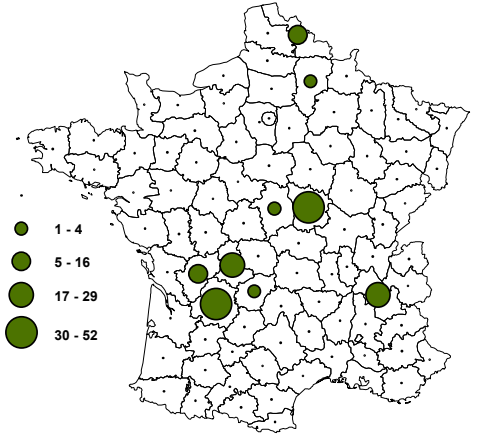

A. Forges in 1789

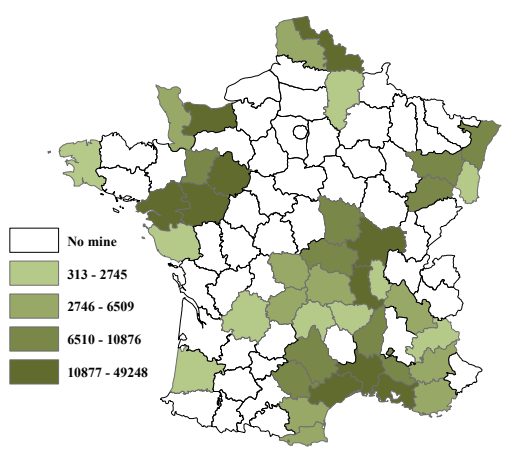

C. Area Covered by Mines in each department in 1837 .

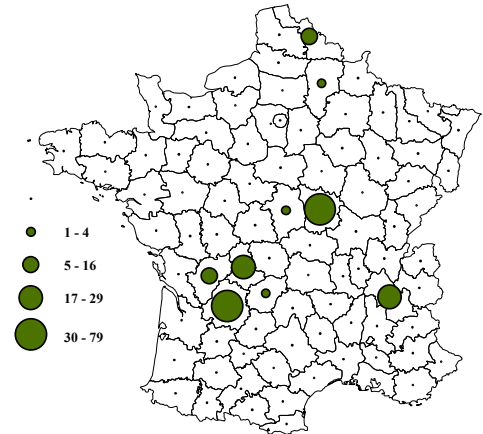

B. Forges in 1811 .

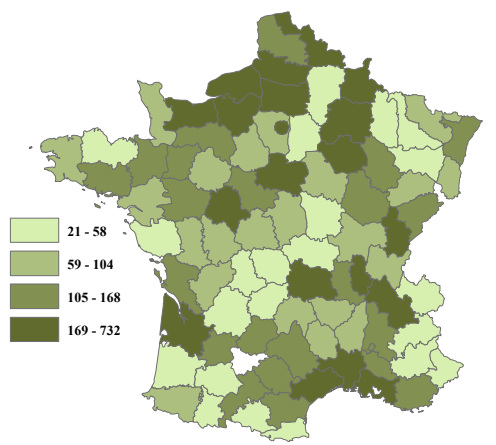

D. Market Integration in the 1790 s.

Figure 8: Early economic development across French departments.

\subsubsection{The Presence of Raw Material}

The diffusion of the steam engine across French departments could have been impacted by the presence of raw material required for industrialization. Moreover the wealth generated by this raw material could have affected human capital. As was shown in the baseline regressions in Tables 7 and 12 , the statistical impact of industrialization on human capital remains intact when one accounts for the share of carboniferous area in each department (Fernihough and O'Rourke, 2014) Nevertheless, the diffusion of the steam engine across France as well as human capital formation could have been affected by the early use of raw material required for industrialization. Nevertheless, the effect of industrialization on human capital in the first stages of the industrial revolution remains nearly intact, economically and statistically, when the number of iron forges in 1789 and 1811 (Panels A and B of Figure 8) in each department (Woronoff, 1997) are accounted for in Table B.6 or when the area covered by coal mines in 1837 (Panel C of Figure 8) in each department is taken into account in Table B.7. 


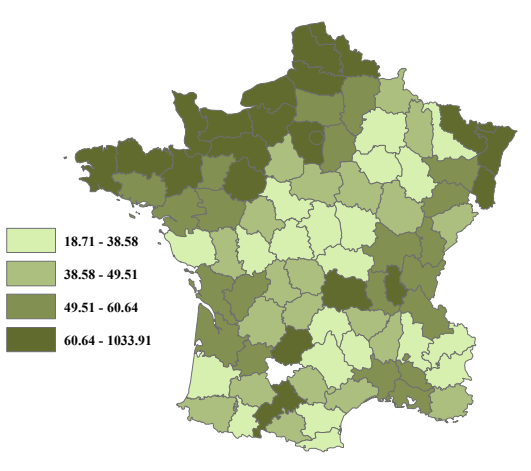

A. Population Density in 1801

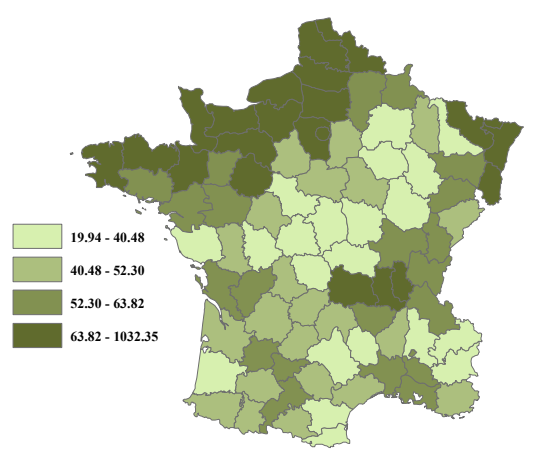

B. Population Density in 1821 .

Figure 9: Population Density in 1801 and 1821

\subsubsection{Early Economic Integration}

Human capital and the number of steam engines across France could have been affected by the degree of economic integration of each department into the French economy in the late 18th century. However, Table B.8 in the Appendix shows that the degree of early market integration of each department, as proxied by the number of firms which were located in one department but sold their products outside that department in the 1790s (Daudin, 2010), and which we graph in Panel D of Figure 8, has no qualitative impact on the effect of industrialization on human capital in the early phase of the industrial revolution. Moreover, as reported in Table B.9, the effect of industrialization on human capital remains intact, economically and statistically. when we account for the existence of a railroad connection in 1860 (Caron, 1997).

\subsubsection{Population Density}

In light of the historical evidence that steam engines were more likely to be located in urban centers (Rosenberg and Trajtenberg, 2004), it is plausible that the potentially endogenous level of population density could have been a factor in the adoption of the steam engine and in the formation of human capital, and could have thus contributed to the relationship between industrialization and human capital formation. Reassuringly, as reported in Table B.10 in the Appendix, population density in each French department in 1801 and 1821 (as shown in Figure 9) has no qualitative impact on the estimated effects and the statistical significance of industrialization on human capital. ${ }^{31}$

\footnotetext{
${ }^{31}$ The Tarn-et-Garonne department was established in 1812 from parts of Aveyron, Gers, Haute-Garonne, Lot and Lot-et-Garonne. Therefore, we lose one observation when population density in 1801 is included in the analysis.
} 


\subsubsection{Past Level of Fertility}

Human capital formation and the number of steam engines across France could have been affected by differential fertility patterns across French departments (reflecting cultural characteristics as well as economic incentives). In particular, conditional on pre-industrial levels of economic and human capital development, departments in which fertility was higher prior to the 1839-1847 industrial survey may have had characteristics that enhanced fertility and lowered the level of investment in human capital (Galor, 2011). However, as reported in Table B.11, although the level of fertility in 1806 has the predicted negative effect on the various measures of human capital formation in the post-1840 period, it has no qualitative impact on the estimated effect of industrialization on human capital formation. ${ }^{32}$

\subsubsection{Investments in Education as a Consumption Good}

The documented rise in human capital formation in the first phase of industrialization is not necessarily indicative of technology-skill complementarity. The positive effect of industrialization on income may have generated a demand for human capital as a consumption good rather than as an investment good. Indeed, as shown in Table B.12, the intensity of industrialization was associated with higher average wages for men and women, as well as children (below age 16), in 1839-1847. Nevertheless, as established in Table B.13, the effect of industrialization on the various measures of human capital remains nearly intact, once one accounts for average male, female and child wages. In particular, the rise in parental income is mostly un-associated with human capital formation whereas the number of steam engines retains its positive impact on education. Moreover, the wages of children are unrelated to human capital formation, perhaps reflecting the compliance with the first limitation on child labor imposed by the 22 March 1841 law.

\subsubsection{The Upper Tail of the Human Capital Distribution in the $18^{\text {th }}$ century}

The number of steam engines and human capital formation in the 19th century might have been affected by the upper tail of the human capital distribution in the previous century. As reported in Table B.14, the upper tail of the human capital distribution in the 18th century, as captured by the number of subscribers to the Quarto edition of the Encyclopédie (Darnton, 1973; Squicciarini and Voigtländer, 2015), has no qualitative impact on the estimated impact of steam engines on human capital formation. Moreover, as established in Table B.1, it has no effect on the impact of the distance from Fresnes-sur-Escaut on the intensive use of the steam engine.

\footnotetext{
${ }^{32} 1806$ is the earliest year for which the level of fertility is available across French departments (Bonneuil, 1997). Accounting for fertility in subsequent years, including the ones contemporaneous to the survey, does not affect the results.
} 


\subsection{Alternative Measures of Human Capital}

\subsubsection{Public Expenditures on Education}

This subsection explores the robustness of the baseline analysis to an alternative proxy of human capital formation, i.e., public expenditures on education. The analysis examines the effect of industrialization on total public expenditures on primary schooling per capita over the 1855-1863 period. As shown in Table D.1 in the Appendix, the OLS regressions in Columns (1)-(7) suggest that the number of steam engines in 1839-1847 mostly has an insignificant association with total public spending on primary schooling. However, in the IV regressions in Columns (8)-(12) which mitigate the effect of omitted variables on the observed relationship and account for the confounding effects of geographical, institutional and pre-industrial characteristics, the effect of industrialization on the share of literate conscripts is found to be positive and significant. ${ }^{33}$ All in all, the IV estimate in Column (8) of Table D.1 indicates that a one-percent increase in the number of steam engines in a department in 1839-1847 increased total public spending on education by $\mathbf{1 . 4 8 \%}$ over the 1855-1863 period.

Moreover, Tables D.2-D.4 assess the relationship between industrialization and public spending on primary schooling per capita by distinguishing expenditures from the three tiers of the French government, i.e., the central state, the departments and the communes, over the 1855-1863 period. In those Tables, the IV regressions in Tables D.2-D.4 indicate a positive and significant effect of industrialization on public spending by the the communes and the departments, but not by central state. This is in line with the historical evidence (e.g., Mayeur (2003)). Indeed, as can be seen in the descriptive statistics reported in Table A.1, most of the public spending on primary schooling was undertaken by the communes in mid-19th century France. It was only after the passing of the laws on mandatory and free schooling in 1881-1882 that the central state begun to finance primary schooling (Franck and Johnson, 2016).

\subsubsection{School Buildings}

This subsection explores the robustness of the baseline analysis to an alternative proxy of human capital formation, i.e., school buildings. The analysis examines the effect of industrialization on the number of school buildings per commune (the lowest tier of the French government) in each department. Surveys carried out in 1850 and 1863 indicate that the average number of school buildings per commune increased from 1.88 in 1850 to 2.19 in 1863 , with large variations in the

\footnotetext{
${ }^{33}$ The F-statistic in the first stage is equal to 13.126. Moreover, the positive and significant effect of industrialization on public expenditures on education per capita during the 1855-1863 period in the IV regressions is corroborated by the reduced form regressions reported in Column (7) of Table B.2 in the Appendix, where the instrument Distance to Fresnes is found to be negatively and significantly correlated with total public spending on education per inhabitant.
} 
number of school buildings across departments. ${ }^{34}$ Since the 1850 and 1863 surveys do not provide information on the size of the school buildings and the number of classrooms, this variable can be viewed as a slightly imprecise measure of human capital formation.

The relationship between industrialization and the number of school buildings in 1850 and 1863 is presented in Tables D.5 and D.6. In Column (1) of both Tables, the unconditional OLS estimate between the number of steam engines in industrial production in 1839-1847 and the number of school buildings in 1840 and 1863 is positive but insignificant. This relationship remains positive and becomes mostly significant once when the confounding effects of exogenous geographical factors (Column (2)), institutional factors (Column (3)) and pre-industrial characteristics (Columns (4)(7)) are taken into account. Finally, in the IV estimations in Columns (8)-(12) which account for the confounding effects of geographical, institutional, and demographic characteristics, the number of steam engines in 1839-1847 is found to have a positive and mostly significant impact on the number of school buildings in 1850 and 1863. The IV estimates in Column (8) of Tables D.5 and D.6 suggest that a one-percent increase in the number of steam engines in a department in 18391847 increased the number of primary schools by $\mathbf{1 6 . 7 \%}$ in 1850 and $\mathbf{1 4 . 4 \%}$ in $1863 .{ }^{35}$ As such, if a department had increased its number of steam engines in 1839-1847 from the 25 th percentile (4) to the 75 th percentile (31), this 675 percent inerease in the number of steam engines would increase the number of school buildings by 112.73 in 185097.20 in 1863 (relative to a sample mean of 1.88 and a standard deviation of 1.34 in 1850 and a mean of 2.19 and a standard deviation of 2.56 in $1863) \cdot{ }^{36}$

Moreover, in the IV regressions in Tables D.5 and D.6, there is a positive and significant association between the distance to Paris and the number of school buildings. There is also a negative and significant correlation between border departments and the number of school buildings. However other characteristics of departments such as latitude, land suitability, the share of carboniferous area in the department, maritime departments, as well as pre-industrial human capital and economic development, do not have any a systematically significant effect on school buildings in the IV regressions.

\footnotetext{
${ }^{34}$ French communes had been compelled by law to host at least one school building in their jurisdiction since 1833.

${ }^{35}$ The F-statistic in the first stage is equal to 13.126 in Column (8), i.e., in the absence of pre-industrial controls. Furthermore, the IV coefficient in each specification is larger than the OLS coefficient, which can probably be attributed to measurement error in the explanatory variable - the number of steam engines.

${ }^{36}$ The F-statistic in the first stage is equal to $\mathbf{1 3 . 1 2 6}$ in the absence of pre-industrial controls. Furthermore, the IV eoefficient in each specification is larger than the OLS coefficient, which can probably be attributed to measurement error in the explanatory variable - the number of steam engines. In addition the positive and significant effect of industrialization on the number of school buildings per commune in 1850 and 1863 in the IV regressions is corroborated by the reduced form regressions reported in Columns (8)-(9) of Table B.2 in the Appendix, where the instrument Distance to Fresnes is found to be negatively and significantly correlated with the number of school buildings per eommune.
} 


\section{Concluding Remarks}

The research explores the effect of industrialization on human capital formation. Exploiting exogenous regional variations in the adoption of steam engines across France, the study establishes that in contrast to conventional wisdom that views early industrialization as a predominantly deskilling process, the industrial revolution was conducive for human capital formation, generating broad increases in literacy rates and education attainment.

In particular, the study established that during the first phase of industrialization in France, a French department that would have experienced an increase in the number of steam engines from the 25 th percentile to the 75 th percentile of the distribution (during the 1839-1847 period), would have witnessed: (i) a $\mathbf{8 0 . 3 3 \%}$ percentage point increase in the share of literate conscripts in 1859-1868, relative to sample mean of $74 \%$, (ii) an additional $\mathbf{7 8 . 9 8}$ pupils (per 10,000 inhabitants) relative to a sample mean of 1179 in 1863, (iii) an additional $\mathbf{6 0 . 3}$ apprentices (per 10,000 inhabitants) relative to a sample mean of 2.71 in 1863, and (iv) an additional $\mathbf{1 9 1 . 0 3}$ teachers in 1863, relative to a sample mean of 1243 .

The research thus lends further credence to the emerging view that human capital was instru-

mental in the process of industrialization, governing the pace of the transition from stagnation to growth and comparative economic development across the world. 
Table 9: The effect of industrialization on the share of pupils in the population in 1840

\begin{tabular}{|c|c|c|c|c|c|c|c|c|c|c|c|c|}
\hline & $\begin{array}{l}1) \\
\text { OLS }\end{array}$ & $\begin{array}{c}(2) \\
\text { OLS }\end{array}$ & $\begin{array}{c}(3) \\
\text { OLS }\end{array}$ & $\begin{array}{c}(4) \\
\text { OLS }\end{array}$ & $\begin{array}{c}(5) \\
\text { OLS }\end{array}$ & $\begin{array}{l}(6) \\
\text { OLS }\end{array}$ & $\begin{array}{l}(7) \\
\text { OLS }\end{array}$ & $\begin{array}{l}\text { (8) } \\
\text { IV }\end{array}$ & $\begin{array}{l}(9) \\
\text { IV }\end{array}$ & $\begin{array}{c}\text { (10) } \\
\text { IV }\end{array}$ & $\begin{array}{l}\text { (11) } \\
\text { IV }\end{array}$ & $\begin{array}{c}(12) \\
\text { IV }\end{array}$ \\
\hline & \multicolumn{12}{|c|}{ Pupils per 10,000 inhabitants, 1840} \\
\hline Number of Steam Engines & $\begin{array}{l}0.0814^{* *} \\
{[0.0352]}\end{array}$ & $\begin{array}{l}0.0862^{* *} \\
{[0.0378]}\end{array}$ & $\begin{array}{l}0.0837 * * \\
{[0.0402]}\end{array}$ & $\begin{array}{c}0.0740^{* *} \\
{[0.0326]}\end{array}$ & $\begin{array}{l}0.103^{* *} \\
{[0.0411]}\end{array}$ & $\begin{array}{l}0.0859^{*} \\
{[0.0437]}\end{array}$ & $\begin{array}{l}0.0810^{* *} \\
{[0.0383]}\end{array}$ & $\begin{array}{c}0.291 * * * \\
{[0.0778]}\end{array}$ & $\begin{array}{l}0.225^{* * *} \\
{[0.0651]}\end{array}$ & $\begin{array}{l}0.297^{* * *} \\
{[0.0705]}\end{array}$ & $\begin{array}{l}0.338^{* * *} \\
{[0.0941]}\end{array}$ & $\begin{array}{c}0.258^{* * *} \\
{[0.0740]}\end{array}$ \\
\hline Average Rainfall & & $\begin{array}{c}0.663^{* *} \\
{[0.259]}\end{array}$ & $\begin{array}{c}0.797^{* * *} \\
{[0.257]}\end{array}$ & $\begin{array}{c}0.384 \\
{[0.256]}\end{array}$ & $\begin{array}{c}0.757^{* * * *} \\
{[0.251]}\end{array}$ & $\begin{array}{c}0.781^{* * *} \\
{[0.274]}\end{array}$ & $\begin{array}{c}0.376 \\
{[0.267]}\end{array}$ & $\begin{array}{c}1.014^{* * *} \\
{[0.257]}\end{array}$ & $\begin{array}{c}0.616^{* *} \\
{[0.300]}\end{array}$ & $\begin{array}{c}0.911^{* * *} \\
{[0.245]}\end{array}$ & $\begin{array}{c}0.776^{* * *} \\
{[0.269]}\end{array}$ & $\begin{array}{c}0.445 \\
{[0.282]}\end{array}$ \\
\hline Average Temperature & & $\begin{array}{c}-2.165^{* * * *} \\
{[0.377]}\end{array}$ & $\begin{array}{c}-1.861 * * * \\
{[0.367]}\end{array}$ & $\begin{array}{c}-0.917^{* *} \\
{[0.383]}\end{array}$ & $\begin{array}{c}-1.771 * * * \\
{[0.380]}\end{array}$ & $\begin{array}{c}-1.865 * * * \\
{[0.369]}\end{array}$ & $\begin{array}{c}-0.922 * * \\
{[0.393]}\end{array}$ & $\begin{array}{c}-1.698^{* * * *} \\
{[0.425]}\end{array}$ & $\begin{array}{c}-0.798^{* *} \\
{[0.402]}\end{array}$ & $\begin{array}{c}-1.556^{* * * *} \\
{[0.427]}\end{array}$ & $\begin{array}{c}-1.768^{* * * *} \\
{[0.427]}\end{array}$ & $\begin{array}{c}-0.854^{* *} \\
{[0.387]}\end{array}$ \\
\hline Latitude & & $\begin{array}{c}0.484 \\
{[0.989]}\end{array}$ & $\begin{array}{c}2.001 \\
{[2.487]}\end{array}$ & $\begin{array}{c}0.00508 \\
{[2.358]}\end{array}$ & $\begin{array}{c}1.831 \\
{[2.614]}\end{array}$ & $\begin{array}{c}1.992 \\
{[2.518]}\end{array}$ & $\begin{array}{l}-0.199 \\
{[2.319]}\end{array}$ & $\begin{array}{c}1.031 \\
{[2.401]}\end{array}$ & $\begin{array}{l}-0.275 \\
{[2.222]}\end{array}$ & $\begin{array}{c}0.851 \\
{[2.668]}\end{array}$ & $\begin{array}{c}0.841 \\
{[2.709]}\end{array}$ & $\begin{array}{l}-1.126 \\
{[2.298]}\end{array}$ \\
\hline Land Suitability & & $\begin{array}{c}0.707^{* * * *} \\
{[0.219]}\end{array}$ & $\begin{array}{c}0.481^{* * * *} \\
{[0.166]}\end{array}$ & $\begin{array}{c}0.213 \\
{[0.176]}\end{array}$ & $\begin{array}{c}0.484^{* * * *} \\
{[0.161]}\end{array}$ & $\begin{array}{c}0.482^{* * * *} \\
{[0.166]}\end{array}$ & $\begin{array}{c}0.221 \\
{[0.178]}\end{array}$ & $\begin{array}{c}0.412^{* *} \\
{[0.161]}\end{array}$ & $\begin{array}{c}0.174 \\
{[0.165]}\end{array}$ & $\begin{array}{c}0.428^{* * * *} \\
{[0.150]}\end{array}$ & $\begin{array}{c}0.422^{* * * *} \\
{[0.157]}\end{array}$ & $\begin{array}{c}0.198 \\
{[0.154]}\end{array}$ \\
\hline Share of Carboniferous Area & & & $\begin{array}{c}-0.849^{* * * *} \\
{[0.312]}\end{array}$ & $\begin{array}{c}-0.643^{* *} \\
{[0.283]}\end{array}$ & $\begin{array}{c}-0.918^{* * *} * \\
{[0.311]}\end{array}$ & $\begin{array}{c}-0.851^{* * *} \\
{[0.314]}\end{array}$ & $\begin{array}{c}-0.667^{* *} \\
{[0.292]}\end{array}$ & $\begin{array}{c}-0.907^{* * * *} \\
{[0.290]}\end{array}$ & $\begin{array}{c}-0.692^{* * *} \\
{[0.267]}\end{array}$ & $\begin{array}{c}-1.026^{* * * *} \\
{[0.291]}\end{array}$ & $\begin{array}{c}-0.929^{* * * *} \\
{[0.306]}\end{array}$ & $\begin{array}{c}-0.747^{* * *} * \\
{[0.275]}\end{array}$ \\
\hline Maritime Department & & & $\begin{array}{l}-0.0573 \\
{[0.0903]}\end{array}$ & $\begin{array}{l}-0.158 \\
{[0.103]}\end{array}$ & $\begin{array}{l}-0.0668 \\
{[0.0938]}\end{array}$ & $\begin{array}{l}-0.0551 \\
{[0.0903]}\end{array}$ & $\begin{array}{l}-0.151 \\
{[0.106]}\end{array}$ & $\begin{array}{c}-0.210^{*} \\
{[0.128]}\end{array}$ & $\begin{array}{c}-0.284^{* *} \\
{[0.128]}\end{array}$ & $\begin{array}{c}-0.207^{*} \\
{[0.123]}\end{array}$ & $\begin{array}{l}-0.184 \\
{[0.133]}\end{array}$ & $\begin{array}{c}-0.233^{*} \\
{[0.121]}\end{array}$ \\
\hline Border Department & & & $\begin{array}{c}0.0669 \\
{[0.0882]}\end{array}$ & $\begin{array}{c}-0.118 \\
{[0.0789]}\end{array}$ & $\begin{array}{c}0.0867 \\
{[0.0872]}\end{array}$ & $\begin{array}{c}0.0654 \\
{[0.0884]}\end{array}$ & $\begin{array}{c}-0.108 \\
{[0.0882]}\end{array}$ & $\begin{array}{l}0.0218 \\
{[0.119]}\end{array}$ & $\begin{array}{c}-0.124 \\
{[0.0882]}\end{array}$ & $\begin{array}{l}0.0646 \\
{[0.107]}\end{array}$ & $\begin{array}{c}-0.00379 \\
{[0.125]}\end{array}$ & $\begin{array}{c}-0.110 \\
{[0.0968]}\end{array}$ \\
\hline Distance to Paris & & & $\begin{array}{c}0.000361 \\
{[0.000698]}\end{array}$ & $\begin{array}{c}0.000283 \\
{[0.000629]}\end{array}$ & $\begin{array}{c}0.000381 \\
{[0.000717]}\end{array}$ & $\begin{array}{c}0.000366 \\
{[0.000709]}\end{array}$ & $\begin{array}{c}0.000245 \\
{[0.000628]}\end{array}$ & $\begin{array}{c}0.000474 \\
{[0.000716]}\end{array}$ & $\begin{array}{c}0.000410 \\
{[0.000593]}\end{array}$ & $\begin{array}{c}0.000494 \\
{[0.000744]}\end{array}$ & $\begin{array}{c}0.000550 \\
{[0.000779]}\end{array}$ & $\begin{array}{c}0.000288 \\
{[0.000616]}\end{array}$ \\
\hline Paris and Suburbs & & & $\begin{array}{c}0.170 \\
{[0.227]}\end{array}$ & $\begin{array}{c}0.103 \\
{[0.141]}\end{array}$ & $\begin{array}{l}0.181 \\
{[0.200]}\end{array}$ & $\begin{array}{c}0.172 \\
{[0.224]}\end{array}$ & $\begin{array}{l}0.0911 \\
{[0.142]}\end{array}$ & $\begin{array}{c}0.100 \\
{[0.306]}\end{array}$ & $\begin{array}{c}0.140 \\
{[0.200]}\end{array}$ & $\begin{array}{c}0.129 \\
{[0.249]}\end{array}$ & $\begin{array}{l}0.126 \\
{[0.253]}\end{array}$ & $\begin{array}{l}0.0678 \\
{[0.191]}\end{array}$ \\
\hline Grooms who Signed their Marriage License, $1786-1790$ & & & & $\begin{array}{c}1.147^{* * * *} \\
{[0.278]}\end{array}$ & & & $\begin{array}{c}1.119^{* * *} \\
{[0.292]}\end{array}$ & & $\begin{array}{c}1.074^{* * * *} \\
{[0.300]}\end{array}$ & & & $\begin{array}{c}1.018^{* * *} * \\
{[0.293]}\end{array}$ \\
\hline University & & & & & $\begin{array}{l}-0.172^{*} \\
{[0.101]}\end{array}$ & & $\begin{array}{c}-0.0632 \\
{[0.101]}\end{array}$ & & & $\begin{array}{c}-0.318^{* * * *} \\
{[0.117]}\end{array}$ & & $\begin{array}{l}-0.172 \\
{[0.105]}\end{array}$ \\
\hline Urban Population in 1700 & & & & & & $\begin{array}{c}-0.00400 \\
{[0.0273]}\end{array}$ & $\begin{array}{c}-0.000106 \\
{[0.0259]}\end{array}$ & & & & $\begin{array}{c}-0.0644^{*} \\
{[0.0337]}\end{array}$ & $\begin{array}{l}-0.0358 \\
{[0.0280]}\end{array}$ \\
\hline Observations & $\begin{array}{c}\text { Adjusted R2 } \\
85\end{array}$ & $\begin{array}{c}0.039 \\
85\end{array}$ & $\begin{array}{c}0.479 \\
85\end{array}$ & $\begin{array}{c}0.515 \\
79 \\
\end{array}$ & $\begin{array}{c}0.661 \\
85 \\
\end{array}$ & $\begin{array}{c}0.527 \\
85 \\
\end{array}$ & $\begin{array}{c}0.509 \\
79 \\
\end{array}$ & $\begin{array}{c}0.652 \\
85 \\
\end{array}$ & 79 & 85 & 85 & 79 \\
\hline & & & & First sta & ge: the instr & umented var & able is Hors & Power of $\mathrm{St}$ & eam Engines & & & \\
\hline $\begin{array}{l}\text { Deviation from Wheat Prices in 1835-1838 } \\
\text { (baseline 1810-1829) }\end{array}$ & & & & & & & & $\begin{array}{c}-0.00687^{* * * *} \\
{[0.00250]} \\
-1.633^{* * *} * \\
{[0.481]}\end{array}$ & $\begin{array}{c}-0.00682^{* *} \\
{[0.00266]} \\
-1.608^{* * *} \\
{[0.527]}\end{array}$ & $\begin{array}{c}-0.00702^{* * *} * \\
{[0.00211]} \\
-1.571^{* * *} \\
{[0.493]}\end{array}$ & $\begin{array}{c}-0.00585^{* *} \\
{[0.00245]} \\
-1.463^{* * *} \\
{[0.511]}\end{array}$ & $\begin{array}{c}-0.00610^{* * * *} \\
{[0.00230]} \\
-1.401^{* *} * \\
{[0.557]}\end{array}$ \\
\hline $\begin{array}{l}\text { F-stat ( } 1^{\text {st }} \text { stage) } \\
\text { Prob J-Stat }\end{array}$ & & & & & & & & $\begin{array}{c}13.126 \\
0.310\end{array}$ & $\begin{array}{c}10.072 \\
0.309\end{array}$ & $\begin{array}{c}14.403 \\
0.342\end{array}$ & $\begin{array}{l}8.658 \\
0.301\end{array}$ & $\begin{array}{l}8.043 \\
0.335\end{array}$ \\
\hline
\end{tabular}

Note: The explanatory variables except the dummies are in logarithm. The aerial distances are measured in kilometers. Robust standard errors are reported in brackets. $* * *$ indicates significance at the $1 \%$-level, ** indicates significance at the $5 \%$-level, $*$ indicates significance at the $10 \%-l$ evel. 
Table 10: The effect of industrialization on the share of pupils in the population in 1863

\begin{tabular}{|c|c|c|c|c|c|c|c|c|c|c|c|c|}
\hline & (1) & (2) & (3) & (4) & (5) & (6) & (7) & $(8)$ & (9) & (10) & (11) & (12) \\
\hline & OLS & OLS & OLS & OLS & OLS & OLS & OLS & IV & IV & IV & IV & IV \\
\hline & \multicolumn{12}{|c|}{ Pupils per 10,000 inhabitants, 1863} \\
\hline Number of Steam Engines & $\begin{array}{l}0.0389^{*} \\
{[0.0199]}\end{array}$ & $\begin{array}{l}0.0383^{*} \\
{[0.0213]}\end{array}$ & $\begin{array}{l}0.0374^{*} \\
{[0.0220]}\end{array}$ & $\begin{array}{l}0.0350^{*} \\
{[0.0201]}\end{array}$ & $\begin{array}{l}0.0401^{*} \\
{[0.0231]}\end{array}$ & $\begin{array}{c}0.0488^{* *} \\
{[0.0223]}\end{array}$ & $\begin{array}{l}0.0423^{*} \\
{[0.0220]}\end{array}$ & $\begin{array}{c}0.117^{* * *} \\
{[0.0323]}\end{array}$ & $\begin{array}{c}0.101 * * * \\
{[0.0322]}\end{array}$ & $\begin{array}{c}0.119^{* * * *} \\
{[0.0316]}\end{array}$ & $\begin{array}{c}0.149^{* * *} \\
{[0.0399]}\end{array}$ & $\begin{array}{c}0.128^{* * *} \\
{[0.0375]}\end{array}$ \\
\hline Average Rainfall & & $\begin{array}{c}0.383^{* * *} \\
{[0.136]}\end{array}$ & $\begin{array}{c}0.501 \text { *** } \\
{[0.126]}\end{array}$ & $\begin{array}{c}0.386^{* * * *} \\
{[0.138]}\end{array}$ & $\begin{array}{c}0.495^{* * * *} \\
{[0.128]}\end{array}$ & $\begin{array}{c}0.421 * * * \\
{[0.146]}\end{array}$ & $\begin{array}{l}0.302^{*} \\
{[0.165]}\end{array}$ & $\begin{array}{c}0.584^{* * * *} \\
{[0.122]}\end{array}$ & $\begin{array}{c}0.488^{* * * *} \\
{[0.152]}\end{array}$ & $\begin{array}{c}0.558^{* * * *} \\
{[0.120]}\end{array}$ & $\begin{array}{c}0.419^{* * *} \\
{[0.139]}\end{array}$ & $\begin{array}{c}0.335^{* *} \\
{[0.165]}\end{array}$ \\
\hline Average Temperature & & $\begin{array}{c}-1.198^{* * *} \\
{[0.222]}\end{array}$ & $\begin{array}{c}-1.057^{* * * *} \\
{[0.206]}\end{array}$ & $\begin{array}{c}-0.759^{* * *} \\
{[0.198]}\end{array}$ & $\begin{array}{c}-1.044^{* * * *} \\
{[0.213]}\end{array}$ & $\begin{array}{c}-1.082^{* * * *} \\
{[0.204]}\end{array}$ & $\begin{array}{c}-0.778^{* * *} \\
{[0.193]}\end{array}$ & $\begin{array}{c}-0.995^{* * *} \\
{[0.226]}\end{array}$ & $\begin{array}{c}-0.707^{* * *} \\
{[0.217]}\end{array}$ & $\begin{array}{c}-0.957^{* * *} \\
{[0.228]}\end{array}$ & $\begin{array}{c}-1.043^{* * *} \\
{[0.223]}\end{array}$ & $\begin{array}{c}-0.745^{* * * *} \\
{[0.200]}\end{array}$ \\
\hline Latitude & & $\begin{array}{c}0.396 \\
{[0.561]}\end{array}$ & $\begin{array}{l}-0.278 \\
{[1.275]}\end{array}$ & $\begin{array}{l}-1.344 \\
{[1.172]}\end{array}$ & $\begin{array}{l}-0.302 \\
{[1.309]}\end{array}$ & $\begin{array}{l}-0.322 \\
{[1.366]}\end{array}$ & $\begin{array}{l}-1.415 \\
{[1.229]}\end{array}$ & $\begin{array}{l}-0.650 \\
{[1.190]}\end{array}$ & $\begin{array}{l}-1.467 \\
{[1.099]}\end{array}$ & $\begin{array}{c}-0.701 \\
{[1.290]}\end{array}$ & $\begin{array}{l}-0.781 \\
{[1.401]}\end{array}$ & $\begin{array}{l}-1.865 \\
{[1.193]}\end{array}$ \\
\hline Land Suitability & & $\begin{array}{c}0.360^{* * * *} \\
{[0.126]}\end{array}$ & $\begin{array}{l}0.235^{* *} \\
{[0.0925]}\end{array}$ & $\begin{array}{c}0.149 \\
{[0.0938]}\end{array}$ & $\begin{array}{l}0.236^{* *} \\
{[0.0919]}\end{array}$ & $\begin{array}{c}0.240^{* * * *} \\
{[0.0872]}\end{array}$ & $\begin{array}{c}0.147 \\
{[0.0892]}\end{array}$ & $\begin{array}{l}0.209^{* *} \\
{[0.0863]}\end{array}$ & $\begin{array}{c}0.131 \\
{[0.0887]}\end{array}$ & $\begin{array}{c}0.213^{* * *} * \\
{[0.0822]}\end{array}$ & $\begin{array}{c}0.216^{* * *} \\
{[0.0762]}\end{array}$ & $\begin{array}{c}0.136^{*} \\
{[0.0766]}\end{array}$ \\
\hline Share of Carboniferous Area & & & $\begin{array}{c}-0.449^{* *} \\
{[0.173]}\end{array}$ & $\begin{array}{c}-0.416^{* *} \\
{[0.167]}\end{array}$ & $\begin{array}{c}-0.459^{* *} \\
{[0.177]}\end{array}$ & $\begin{array}{c}-0.455^{* * *} \\
{[0.165]}\end{array}$ & $\begin{array}{c}-0.396^{* *} \\
{[0.163]}\end{array}$ & $\begin{array}{c}-0.471^{* * *} \\
{[0.152]}\end{array}$ & $\begin{array}{c}-0.438^{* * *} \\
{[0.153]}\end{array}$ & $\begin{array}{c}-0.503^{* * *} \\
{[0.154]}\end{array}$ & $\begin{array}{c}-0.487^{* * * *} \\
{[0.146]}\end{array}$ & $\begin{array}{c}-0.435^{* * *} * \\
{[0.148]}\end{array}$ \\
\hline Maritime Department & & & $\begin{array}{c}0.0103 \\
{[0.0487]}\end{array}$ & $\begin{array}{l}-0.0176 \\
{[0.0535]}\end{array}$ & $\begin{array}{l}0.00890 \\
{[0.0502]}\end{array}$ & $\begin{array}{c}0.0213 \\
{[0.0485]}\end{array}$ & $\begin{array}{c}-0.00360 \\
{[0.0506]}\end{array}$ & $\begin{array}{l}-0.0484 \\
{[0.0572]}\end{array}$ & $\begin{array}{l}-0.0730 \\
{[0.0598]}\end{array}$ & $\begin{array}{l}-0.0479 \\
{[0.0578]}\end{array}$ & $\begin{array}{l}-0.0301 \\
{[0.0600]}\end{array}$ & $\begin{array}{l}-0.0431 \\
{[0.0574]}\end{array}$ \\
\hline Border Department & & & $\begin{array}{c}0.0419 \\
{[0.0482]}\end{array}$ & $\begin{array}{l}-0.0256 \\
{[0.0488]}\end{array}$ & $\begin{array}{c}0.0447 \\
{[0.0494]}\end{array}$ & $\begin{array}{l}0.0345 \\
{[0.0488]}\end{array}$ & $\begin{array}{l}-0.0413 \\
{[0.0542]}\end{array}$ & $\begin{array}{l}0.0247 \\
{[0.0564]}\end{array}$ & $\begin{array}{l}-0.0281 \\
{[0.0502]}\end{array}$ & $\begin{array}{c}0.0358 \\
{[0.0555]}\end{array}$ & $\begin{array}{l}0.00691 \\
{[0.0602]}\end{array}$ & $\begin{array}{l}-0.0426 \\
{[0.0571]}\end{array}$ \\
\hline Distance to Paris & & & $\begin{array}{c}-0.000273 \\
{[0.000365]}\end{array}$ & $\begin{array}{c}-0.000408 \\
{[0.000339]}\end{array}$ & $\begin{array}{r}-0.000270 \\
{[0.000373]}\end{array}$ & $\begin{array}{c}-0.000250 \\
{[0.000384]}\end{array}$ & $\begin{array}{c}-0.000395 \\
{[0.000350]}\end{array}$ & $\begin{array}{c}-0.000229 \\
{[0.000353]}\end{array}$ & $\begin{array}{c}-0.000352 \\
{[0.000318]}\end{array}$ & $\begin{array}{c}-0.000224 \\
{[0.000368]}\end{array}$ & $\begin{array}{l}-0.000176 \\
{[0.000390]}\end{array}$ & $\begin{array}{l}-0.000375 \\
{[0.000335]}\end{array}$ \\
\hline Paris and Suburbs & & & $\begin{array}{l}-0.0311 \\
{[0.158]}\end{array}$ & $\begin{array}{l}0.0239 \\
{[0.103]}\end{array}$ & $\begin{array}{l}-0.0297 \\
{[0.154]}\end{array}$ & $\begin{array}{l}-0.0218 \\
{[0.137]}\end{array}$ & $\begin{array}{l}0.00594 \\
{[0.0989]}\end{array}$ & $\begin{array}{l}-0.0581 \\
{[0.185]}\end{array}$ & $\begin{array}{l}0.0404 \\
{[0.131]}\end{array}$ & $\begin{array}{l}-0.0507 \\
{[0.169]}\end{array}$ & $\begin{array}{l}-0.0404 \\
{[0.145]}\end{array}$ & $\begin{array}{c}-0.00536 \\
{[0.121]}\end{array}$ \\
\hline Grooms who Signed their Marriage License, $1786-1790$ & & & & $\begin{array}{c}0.351^{* * *} \\
{[0.130]}\end{array}$ & & & $\begin{array}{c}0.380^{* * *} \\
{[0.141]}\end{array}$ & & $\begin{array}{c}0.319^{* *} \\
{[0.136]}\end{array}$ & & & $\begin{array}{c}0.331^{* *} \\
{[0.140]}\end{array}$ \\
\hline University & & & & & $\begin{array}{l}-0.0246 \\
{[0.0533]}\end{array}$ & & $\begin{array}{l}0.0357 \\
{[0.0521]}\end{array}$ & & & $\begin{array}{l}-0.0835 \\
{[0.0607]}\end{array}$ & & $\begin{array}{l}-0.0172 \\
{[0.0558]}\end{array}$ \\
\hline Urban Population in 1700 & & & & & & $\begin{array}{l}-0.0205 \\
{[0.0157]}\end{array}$ & $\begin{array}{l}-0.0218 \\
{[0.0163]}\end{array}$ & & & & $\begin{array}{c}-0.0446^{* *} \\
{[0.0187]}\end{array}$ & $\begin{array}{c}-0.0391^{* *} \\
{[0.0170]}\end{array}$ \\
\hline Adjusted R2 & 0.028 & 0.513 & 0.544 & 0.610 & 0.539 & 0.551 & 0.613 & & & & & \\
\hline Observations & 85 & 85 & 85 & 79 & 85 & 85 & 79 & 85 & 79 & 85 & 85 & 79 \\
\hline \multicolumn{13}{|c|}{ First stage: the instrumented variable is Horse Power of Steam Engines } \\
\hline Distance to Fresnes & & & & & & & & $\begin{array}{c}-0.00687^{* * * *} \\
{[0.00250]}\end{array}$ & $\begin{array}{c}-0.00682^{* *} \\
{[0.00266]}\end{array}$ & $\begin{array}{c}-0.00702^{* * * *} \\
{[0.00211]}\end{array}$ & $\begin{array}{c}-0.00585^{* *} \\
{[0.00245]}\end{array}$ & $\begin{array}{c}-0.00610^{* * *} \\
{[0.00230]}\end{array}$ \\
\hline $\begin{array}{l}\text { Deviation from Wheat Prices in } 1835-1838 \\
\text { (baseline } 1810-1829 \text { ) }\end{array}$ & & & & & & & & $\begin{array}{c}-1.633^{* * * *} \\
{[0.481]}\end{array}$ & $\begin{array}{c}-1.608^{* * *} \\
{[0.527]}\end{array}$ & $\begin{array}{c}-1.571^{* * *} \\
{[0.493]}\end{array}$ & $\begin{array}{c}-1.463^{* * *} \\
{[0.511]}\end{array}$ & $\begin{array}{c}-1.401^{* *} \\
{[0.557]}\end{array}$ \\
\hline F-stat ( $1^{\text {st }}$ stage $)$ & & & & & & & & 13.126 & 10.072 & 14.403 & 8.658 & 8.043 \\
\hline Prob J-Stat & & & & & & & & 0.151 & 0.188 & 0.167 & 0.160 & 0.196 \\
\hline
\end{tabular}

Note: The explanatory variables except the dummies are in logarithm. The aerial distances are measured in kilometers. Robust standard errors are reported in brackets. $* * *$ indicates significance at the $1 \%$-level, $* *$ indicates significance at the $5 \%$-level, $*$ indicates significance at the $10 \%$-level. 
Table 11: The effect of industrialization on the share of apprentices in the population in 1863

\begin{tabular}{|c|c|c|c|c|c|c|c|c|c|c|c|c|}
\hline & (1) & $(2)$ & (3) & $\begin{array}{l}(4) \\
\end{array}$ & (5) & (6) & $7(7)$ & $(8)$ & $\begin{array}{lll}(9) \\
\end{array}$ & (10) & (11) & $(12)$ \\
\hline & \multirow{2}{*}{\multicolumn{12}{|c|}{ Apprentices per 10,000 inhabitants, 1863}} \\
\hline & & & & & & & & & & & & \\
\hline \multirow[t]{2}{*}{ Number of Steam Engines } & $0.376^{* * * *}$ & $0.394^{* * *}$ & $0.351^{* * * *}$ & $0.326^{* * * *}$ & $0.349^{* * *}$ & $0.329^{* * *}$ & $0.308^{* * * *}$ & $0.603^{* * * *}$ & $0.588^{* * * *}$ & $0.612^{* * * *}$ & $0.622^{* * * *}$ & $0.621^{* * * *}$ \\
\hline & {$[0.0685]$} & {$[0.0921]$} & {$[0.0759]$} & {$[0.0840]$} & {$[0.0808]$} & {$[0.0823]$} & {$[0.0940]$} & {$[0.159]$} & {$[0.174]$} & {$[0.161]$} & {$[0.205]$} & {$[0.220]$} \\
\hline \multirow[t]{2}{*}{ Average Rainfall } & & 0.644 & 0.797 & 0.610 & 0.802 & 0.949 & 0.736 & $1.062^{* *}$ & $1.012^{*}$ & $1.011^{* *}$ & $0.944^{*}$ & 0.858 \\
\hline & & {$[0.597]$} & {$[0.554]$} & {$[0.640]$} & {$[0.558]$} & {$[0.583]$} & {$[0.699]$} & {$[0.488]$} & {$[0.604]$} & {$[0.470]$} & {$[0.535]$} & {$[0.643]$} \\
\hline \multirow[t]{2}{*}{ Average Temperature } & & -0.107 & 0.927 & 1.020 & 0.915 & 0.975 & 1.054 & 1.125 & 1.225 & 1.207 & 1.089 & 1.174 \\
\hline & & {$[0.543]$} & {$[0.726]$} & {$[0.897]$} & {$[0.725]$} & {$[0.744]$} & {$[0.928]$} & {$[0.763]$} & {$[0.897]$} & {$[0.776]$} & {$[0.767]$} & {$[0.903]$} \\
\hline \multirow[t]{2}{*}{ Latitude } & & 2.824 & -0.765 & -0.545 & -0.742 & -0.681 & -0.211 & -1.946 & -1.029 & -2.068 & -2.017 & -1.849 \\
\hline & & {$[2.323]$} & {$[4.520]$} & {$[5.567]$} & [4.495] & {$[4.646]$} & {$[5.678]$} & {$[4.320]$} & {$[5.259]$} & {$[4.270]$} & {$[4.287]$} & [5.158] \\
\hline \multirow[t]{2}{*}{ Land Suitability } & & $0.476^{*}$ & 0.147 & 0.133 & 0.146 & 0.138 & 0.128 & 0.0627 & 0.0644 & 0.0700 & 0.0690 & 0.0881 \\
\hline & & {$[0.283]$} & {$[0.344]$} & {$[0.407]$} & {$[0.346]$} & {$[0.339]$} & {$[0.414]$} & {$[0.326]$} & {$[0.384]$} & {$[0.337]$} & {$[0.334]$} & {$[0.405]$} \\
\hline \multirow[t]{2}{*}{ Share of Carboniferous Area } & & & -0.379 & -0.279 & -0.370 & -0.367 & -0.279 & -0.449 & -0.365 & -0.515 & -0.459 & -0.420 \\
\hline & & & {$[0.497]$} & {$[0.555]$} & {$[0.510]$} & {$[0.486]$} & {$[0.580]$} & [0.550] & {$[0.625]$} & {$[0.562]$} & {$[0.555]$} & {$[0.657]$} \\
\hline \multirow[t]{2}{*}{ Maritime Department } & & & 0.217 & 0.239 & 0.218 & 0.196 & 0.212 & 0.0304 & 0.0214 & 0.0287 & 0.0462 & 0.0681 \\
\hline & & & {$[0.265]$} & {$[0.320]$} & {$[0.268]$} & {$[0.271]$} & {$[0.341]$} & {$[0.262]$} & {$[0.314]$} & {$[0.258]$} & {$[0.259]$} & {$[0.309]$} \\
\hline Border Department & & & $\begin{array}{c}0.936^{* * *} \\
{[0.289]}\end{array}$ & $\begin{array}{c}0.898^{* * * *} \\
{[0.316]}\end{array}$ & $\begin{array}{c}0.933^{* * *} \\
{[0.287]}\end{array}$ & $\begin{array}{c}0.950^{* * *} \\
{[0.297]}\end{array}$ & $\begin{array}{c}0.907^{* * *} \\
{[0.317]}\end{array}$ & $\begin{array}{c}0.881^{* * *} \\
{[0.277]}\end{array}$ & $\begin{array}{c}0.888^{* * * *} \\
{[0.290]}\end{array}$ & $\begin{array}{c}0.903^{* * *} \\
{[0.265]}\end{array}$ & $\begin{array}{c}0.870^{* * * *} \\
{[0.278]}\end{array}$ & $\begin{array}{c}0.902^{* * *} \\
{[0.277]}\end{array}$ \\
\hline \multirow[t]{2}{*}{ Distance to Paris } & & & -0.00141 & -0.00127 & -0.00142 & -0.00146 & -0.00125 & -0.00128 & -0.00105 & -0.00126 & -0.00124 & -0.00117 \\
\hline & & & {$[0.00133]$} & {$[0.00153]$} & [0.00135] & {$[0.00134]$} & {$[0.00152]$} & {$[0.00128]$} & {$[0.00147]$} & {$[0.00126]$} & {$[0.00129]$} & {$[0.00140]$} \\
\hline \multirow[t]{2}{*}{ Paris and Suburbs } & & & 0.772 & 0.464 & 0.771 & 0.755 & 0.502 & 0.687 & 0.529 & 0.701 & 0.700 & 0.461 \\
\hline & & & {$[0.557]$} & {$[0.710]$} & {$[0.561]$} & {$[0.565]$} & {$[0.741]$} & {$[0.579]$} & {$[0.793]$} & {$[0.600]$} & {$[0.577]$} & {$[0.782]$} \\
\hline Grooms who Signed their Marriage License, $1786-1790$ & & & & $\begin{array}{c}0.239 \\
{[0.583]}\end{array}$ & & & $\begin{array}{c}0.232 \\
{[0.593]}\end{array}$ & & $\begin{array}{c}0.111 \\
{[0.566]}\end{array}$ & & & $\begin{array}{l}0.0536 \\
{[0.586]}\end{array}$ \\
\hline \multirow[t]{2}{*}{ University } & & & & & 0.0232 & & 0.0233 & & & -0.173 & & -0.169 \\
\hline & & & & & {$[0.246]$} & & {$[0.290]$} & & & {$[0.257]$} & & {$[0.290]$} \\
\hline Urban Population in 1700 & & & & & & $\begin{array}{c}0.0394 \\
{[0.0574]}\end{array}$ & $\begin{array}{c}0.0304 \\
{[0.0700]}\end{array}$ & & & & $\begin{array}{l}-0.0308 \\
{[0.0815]}\end{array}$ & $\begin{array}{l}-0.0327 \\
{[0.0882]}\end{array}$ \\
\hline Adjusted r2 & 0.256 & 0.257 & 0.366 & 0.303 & 0.357 & 0.361 & 0.283 & & & & & \\
\hline \multirow[t]{2}{*}{ Observations } & 85 & 85 & 85 & 79 & 85 & 85 & 79 & 85 & 79 & 85 & 85 & 79 \\
\hline & \multicolumn{12}{|c|}{ First stage: the instrumented variable is Horse Power of Steam Engines } \\
\hline Distance to Fresnes & & & & & & & & $\begin{array}{l}-0.00687^{* * * *} \\
{[0.00250]}\end{array}$ & $\begin{array}{c}-0.00682^{* * *} \\
{[0.00266]}\end{array}$ & $\begin{array}{c}-0.00702^{* * *} \\
{[0.00211]}\end{array}$ & $\begin{array}{c}-0.00585^{* *} \\
{[0.00245]}\end{array}$ & $\begin{array}{r}-0.00610^{* * *} \\
{[0.00230]}\end{array}$ \\
\hline $\begin{array}{l}\text { Deviation from Wheat Prices in } 1835-1838 \\
\text { (baseline 1810-1829) }\end{array}$ & & & & & & & & $\begin{array}{c}-1.633^{* * *} \\
{[0.481]}\end{array}$ & $\begin{array}{c}-1.608^{* * * *} \\
{[0.527]}\end{array}$ & $\begin{array}{c}-1.571^{* * *} \\
{[0.493]}\end{array}$ & $\begin{array}{c}-1.463^{* * *} \\
{[0.511]}\end{array}$ & $\begin{array}{c}-1.401^{* *} \\
{[0.557]}\end{array}$ \\
\hline F-stat ( $1^{s t}$ stage $)$ & & & & & & & & 13.126 & 10.072 & 14.403 & 8.658 & 8.043 \\
\hline Prob J-Stat & & & & & & & & 0.168 & 0.166 & 0.174 & 0.162 & 0.161 \\
\hline
\end{tabular}

Note: The explanatory variables except the dummies are in logarithm. The aerial distances are measured in kilometers. Robust standard errors are reported in brackets. *** indicates significance at the $1 \%$-level, ** indicates significance at the $5 \%$-level, $*$ indicates significance at the $10 \%$-level. 
Table 12: The effect of industrialization on the share of literate conscripts, 1859-1868

\begin{tabular}{|c|c|c|c|c|c|c|c|c|c|c|c|c|}
\hline & $\begin{array}{l}(1) \\
\text { OLS }\end{array}$ & $\begin{array}{c}(2) \\
\text { OLS }\end{array}$ & $\begin{array}{c}\text { (3) } \\
\text { OLS }\end{array}$ & $\begin{array}{l}(4) \\
\text { OLS }\end{array}$ & $\begin{array}{l}\text { OLS } \\
\text { OLS }\end{array}$ & $\begin{array}{c}6) \\
\text { OLS }\end{array}$ & $\begin{array}{l}(7) \\
\text { OLS }\end{array}$ & $\begin{array}{l}\text { (8) } \\
\text { IV }\end{array}$ & $\begin{array}{l}(9) \\
\text { IV }\end{array}$ & $\begin{array}{l}(10) \\
\text { IV }\end{array}$ & $\begin{array}{l}\text { (11) } \\
\text { IV }\end{array}$ & $\begin{array}{l}(12) \\
\text { IV }\end{array}$ \\
\hline & \multicolumn{12}{|c|}{ Share of Conscripts who could Read and Write, 1859-1868 } \\
\hline Number of Steam Engines & $\begin{array}{c}0.0461^{* *} \\
{[0.0223]}\end{array}$ & $\begin{array}{c}0.0364 \\
{[0.0220]}\end{array}$ & $\begin{array}{c}0.0401 * * \\
{[0.0200]}\end{array}$ & $\begin{array}{l}0.0279^{*} \\
{[0.0154]}\end{array}$ & $\begin{array}{c}0.0464^{* *} \\
{[0.0201]}\end{array}$ & $\begin{array}{c}0.0349 \\
{[0.0220]}\end{array}$ & $\begin{array}{c}0.0274 \\
{[0.0175]}\end{array}$ & $\begin{array}{c}0.119^{* * *} \\
{[0.0375]}\end{array}$ & $\begin{array}{c}0.0762^{* * *} \\
{[0.0302]}\end{array}$ & $\begin{array}{c}0.121^{* * * *} \\
{[0.0352]}\end{array}$ & $\begin{array}{c}0.129^{* * *} \\
{[0.0424]}\end{array}$ & $\begin{array}{c}0.0829 * * * \\
{[0.0319]}\end{array}$ \\
\hline Average Rainfall & & $\begin{array}{l}0.0867 \\
{[0.130]}\end{array}$ & $\begin{array}{c}0.242^{* *} \\
{[0.121]}\end{array}$ & $\begin{array}{l}0.0127 \\
{[0.136]}\end{array}$ & $\begin{array}{l}0.229^{*} \\
{[0.121]}\end{array}$ & $\begin{array}{c}0.278^{* *} \\
{[0.135]}\end{array}$ & $\begin{array}{l}0.0335 \\
{[0.135]}\end{array}$ & $\begin{array}{c}0.325^{* * *} \\
{[0.120]}\end{array}$ & $\begin{array}{l}0.0869 \\
{[0.140]}\end{array}$ & $\begin{array}{c}0.288^{* *} \\
{[0.117]}\end{array}$ & $\begin{array}{c}0.276^{* *} \\
{[0.132]}\end{array}$ & $\begin{array}{l}0.0551 \\
{[0.132]}\end{array}$ \\
\hline Average Temperature & & $\begin{array}{c}-0.810^{* * * *} \\
{[0.211]}\end{array}$ & $\begin{array}{c}-0.540^{* * * *} \\
{[0.175]}\end{array}$ & $\begin{array}{c}-0.0942 \\
{[0.212]}\end{array}$ & $\begin{array}{c}-0.510^{* * * *} \\
{[0.178]}\end{array}$ & $\begin{array}{c}-0.528^{* * *} \\
{[0.176]}\end{array}$ & $\begin{array}{l}-0.0902 \\
{[0.221]}\end{array}$ & $\begin{array}{c}-0.478^{* *} \\
{[0.192]}\end{array}$ & $\begin{array}{l}-0.0564 \\
{[0.209]}\end{array}$ & $\begin{array}{c}-0.427^{* *} \\
{[0.189]}\end{array}$ & $\begin{array}{c}-0.492^{* * *} \\
{[0.190]}\end{array}$ & $\begin{array}{l}-0.0689 \\
{[0.205]}\end{array}$ \\
\hline Latitude & & $\begin{array}{l}-0.237 \\
{[0.613]}\end{array}$ & $\begin{array}{c}0.234 \\
{[1.410]}\end{array}$ & $\begin{array}{l}-0.827 \\
{[1.375]}\end{array}$ & $\begin{array}{c}0.178 \\
{[1.444]}\end{array}$ & $\begin{array}{c}0.254 \\
{[1.381]}\end{array}$ & $\begin{array}{l}-0.850 \\
{[1.346]}\end{array}$ & $\begin{array}{l}-0.136 \\
{[1.234]}\end{array}$ & $\begin{array}{l}-0.916 \\
{[1.190]}\end{array}$ & $\begin{array}{l}-0.199 \\
{[1.320]}\end{array}$ & $\begin{array}{l}-0.174 \\
{[1.295]}\end{array}$ & $\begin{array}{l}-1.140 \\
{[1.197]}\end{array}$ \\
\hline Land Suitability & & $\begin{array}{c}0.367^{* * * *} \\
{[0.108]}\end{array}$ & $\begin{array}{l}0.232 * * * \\
{[0.0803]}\end{array}$ & $\begin{array}{c}0.104 \\
{[0.0898]}\end{array}$ & $\begin{array}{c}0.233 * * * \\
{[0.0794]}\end{array}$ & $\begin{array}{c}0.230^{* * *} \\
{[0.0800]}\end{array}$ & $\begin{array}{c}0.106 \\
{[0.0929]}\end{array}$ & $\begin{array}{c}0.206^{* * *} \\
{[0.0744]}\end{array}$ & $\begin{array}{c}0.0908 \\
{[0.0825]}\end{array}$ & $\begin{array}{c}0.211^{* * *} \\
{[0.0719]}\end{array}$ & $\begin{array}{c}0.208^{* * *} \\
{[0.0744]}\end{array}$ & $\begin{array}{c}0.0986 \\
{[0.0811]}\end{array}$ \\
\hline Share of Carboniferous Area & & & $\begin{array}{c}-0.475^{* *} \\
{[0.220]}\end{array}$ & $\begin{array}{l}-0.364^{*} \\
{[0.206]}\end{array}$ & $\begin{array}{c}-0.498^{* *} \\
{[0.217]}\end{array}$ & $\begin{array}{c}-0.473^{* *} \\
{[0.221]}\end{array}$ & $\begin{array}{c}-0.374^{*} \\
{[0.204]}\end{array}$ & $\begin{array}{c}-0.497^{* *} \\
{[0.197]}\end{array}$ & $\begin{array}{c}-0.380^{* *} \\
{[0.183]}\end{array}$ & $\begin{array}{c}-0.540^{* * * *} \\
{[0.191]}\end{array}$ & $\begin{array}{c}-0.502^{* *} \\
{[0.203]}\end{array}$ & $\begin{array}{c}-0.399^{* *} \\
{[0.181]}\end{array}$ \\
\hline Maritime Department & & & $\begin{array}{l}-0.0788^{*} \\
{[0.0472]}\end{array}$ & $\begin{array}{l}-0.117^{* * *} \\
{[0.0520]}\end{array}$ & $\begin{array}{l}-0.0819^{*} \\
{[0.0491]}\end{array}$ & $\begin{array}{l}-0.0839^{*} \\
{[0.0469]}\end{array}$ & $\begin{array}{c}-0.119^{* * *} \\
{[0.0586]}\end{array}$ & $\begin{array}{l}-0.137^{* *} \\
{[0.0609]}\end{array}$ & $\begin{array}{c}-0.157^{* *} \\
{[0.0613]}\end{array}$ & $\begin{array}{c}-0.136^{* *} \\
{[0.0611]}\end{array}$ & $\begin{array}{l}-0.132^{* * *} \\
{[0.0616]}\end{array}$ & $\begin{array}{l}-0.145^{* * *} \\
{[0.0629]}\end{array}$ \\
\hline Border Department & & & $\begin{array}{c}0.0441 \\
{[0.0530]}\end{array}$ & $\begin{array}{l}-0.0527 \\
{[0.0433]}\end{array}$ & $\begin{array}{c}0.0506 \\
{[0.0529]}\end{array}$ & $\begin{array}{c}0.0475 \\
{[0.0528]}\end{array}$ & $\begin{array}{l}-0.0464 \\
{[0.0447]}\end{array}$ & $\begin{array}{c}0.0269 \\
{[0.0624]}\end{array}$ & $\begin{array}{l}-0.0546 \\
{[0.0444]}\end{array}$ & $\begin{array}{c}0.0421 \\
{[0.0586]}\end{array}$ & $\begin{array}{c}0.0217 \\
{[0.0636]}\end{array}$ & $\begin{array}{l}-0.0472 \\
{[0.0461]}\end{array}$ \\
\hline Distance to Paris & & & $\begin{array}{c}7.95 \mathrm{e}-05 \\
{[0.000413]}\end{array}$ & $\begin{array}{c}2.91 \mathrm{e}-05 \\
{[0.000383]}\end{array}$ & $\begin{array}{c}8.59 \mathrm{e}-05 \\
{[0.000418]}\end{array}$ & $\begin{array}{c}6.92 \mathrm{e}-05 \\
{[0.000409]}\end{array}$ & $\begin{array}{c}1.80 \mathrm{e}-05 \\
{[0.000381]}\end{array}$ & $\begin{array}{c}0.000122 \\
{[0.000393]}\end{array}$ & $\begin{array}{c}6.98 \mathrm{e}-05 \\
{[0.000341]}\end{array}$ & $\begin{array}{c}0.000130 \\
{[0.000399]}\end{array}$ & $\begin{array}{c}0.000138 \\
{[0.000405]}\end{array}$ & $\begin{array}{c}3.14 \mathrm{e}-05 \\
{[0.000345]}\end{array}$ \\
\hline Paris and Suburbs & & & $\begin{array}{c}0.227 * * * \\
{[0.0732]}\end{array}$ & $\begin{array}{c}0.0916 \\
{[0.0626]}\end{array}$ & $\begin{array}{c}0.230^{* * *} \\
{[0.0752]}\end{array}$ & $\begin{array}{c}0.223^{* * *} \\
{[0.0754]}\end{array}$ & $\begin{array}{c}0.0939 \\
{[0.0639]}\end{array}$ & $\begin{array}{l}0.200^{* *} \\
{[0.0929]}\end{array}$ & $\begin{array}{c}0.104 \\
{[0.0771]}\end{array}$ & $\begin{array}{l}0.210^{* *} \\
{[0.0878]}\end{array}$ & $\begin{array}{l}0.205^{* *} \\
{[0.0879]}\end{array}$ & $\begin{array}{c}0.0866 \\
{[0.0752]}\end{array}$ \\
\hline Grooms who Signed their Marriage License, $1786-1790$ & & & & $\begin{array}{c}0.578^{* * *} \\
{[0.150]}\end{array}$ & & & $\begin{array}{c}0.565^{* * *} \\
{[0.156]}\end{array}$ & & $\begin{array}{c}0.555^{* * * *} \\
{[0.149]}\end{array}$ & & & $\begin{array}{c}0.533^{* * *} \\
{[0.145]}\end{array}$ \\
\hline University & & & & & $\begin{array}{l}-0.0568 \\
{[0.0529]}\end{array}$ & & $\begin{array}{l}-0.0224 \\
{[0.0460]}\end{array}$ & & & $\begin{array}{c}-0.113^{* *} \\
{[0.0558]}\end{array}$ & & $\begin{array}{l}-0.0565 \\
{[0.0457]}\end{array}$ \\
\hline Urban Population in 1700 & & & & & & $\begin{array}{l}0.00940 \\
{[0.0141]}\end{array}$ & $\begin{array}{l}0.00570 \\
{[0.0144]}\end{array}$ & & & & $\begin{array}{l}-0.0131 \\
{[0.0150]}\end{array}$ & $\begin{array}{l}-0.00547 \\
{[0.0128]}\end{array}$ \\
\hline Adjusted R2 & 0.046 & 0.289 & 0.396 & 0.560 & 0.397 & 0.391 & 0.549 & & & & & \\
\hline \multirow[t]{2}{*}{ Observations } & 85 & 85 & 85 & 79 & 85 & 85 & 79 & 85 & 79 & 85 & 85 & 79 \\
\hline & \multicolumn{12}{|c|}{ First stage: the instrumented variable is Horse Power of Steam Engines } \\
\hline Distance to Fresnes & & & & & & & & $\begin{array}{c}-0.00687^{* * * *} \\
{[0.00250]}\end{array}$ & $\begin{array}{c}-0.00682^{* *} \\
{[0.00266]}\end{array}$ & $\begin{array}{c}-0.00702^{* * * *} \\
{[0.00211]}\end{array}$ & $\begin{array}{c}-0.00585 * * \\
{[0.00245]}\end{array}$ & $\begin{array}{c}-0.00610^{* * * *} \\
{[0.00230]}\end{array}$ \\
\hline $\begin{array}{l}\text { Deviation from Wheat Prices in } 1835-1838 \\
\text { (baseline 1810-1829) }\end{array}$ & & & & & & & & $\begin{array}{c}-1.633^{* * * *} \\
{[0.481]}\end{array}$ & $\begin{array}{c}-1.608^{* * *} \\
{[0.527]}\end{array}$ & $\begin{array}{c}-1.571^{* * *} \\
{[0.493]}\end{array}$ & $\begin{array}{c}-1.463^{* * *} \\
{[0.511]}\end{array}$ & $\begin{array}{c}-1.401^{* *} \\
{[0.557]}\end{array}$ \\
\hline F-stat $\left(1^{s t}\right.$ stage $)$ & & & & & & & & 13.126 & 10.072 & 14.403 & 8.658 & 8.043 \\
\hline Prob J-Stat & & & & & & & & 0.563 & 0.499 & 0.610 & 0.548 & 0.539 \\
\hline
\end{tabular}

Note: The explanatory variables except the dummies are in logarithm. The aerial distances are measured in kilometers. Robust standard errors are reported in brackets. *** indicates significance at the $1 \%$-level, ** indicates significance at the $5 \%$-level, * indicates significance at the $10 \%$-level. 


\section{References}

Angrist, Joshua D. and Jörn-Steffen Pischke (2009), Mostly Harmless Econometrics: An Empiricist's Companion, Princeton University Press, Princeton, NJ.

Ballot, Charles (1923), L'Introduction du Machinisme dans l'Industrie Française, Slatkine Reprints (1978), Geneva.

Bonneuil, Noël (1997), Transformation of the French Demographic Landscape, 1806-1906, Clarendon Press, Oxford, UK.

Bosker, Maarten, Eltjo Buringh and Jan Luiten van Zanden (2013), 'From Baghdad to London: unravelling urban development in Europe and the Arab world 800-1800', Review of Economics and Statistics 95(4), 1418-1437.

Boucekkine, Raouf, David de la Croix and Dominique Peeters (2007), 'Early literacy achievements, population density, and the transition to modern growth', Journal of the European Economic Association $\mathbf{5}(1), 183-226$.

Bresnahan, Timothy F. and Manuel Trajtenberg (1995), 'General purpose technologies: engines of growth?', Journal of Econometrics 65(1), 83-108.

Cameron, Rondo and Larry Neal (2002), A Concise Economic History of the World from Paleolithic Times to the Present, 4th edn, Oxford University Press, New York.

Caron, François (1997), Histoire des chemins de fer en France: 1740-1883, Fayard, Paris.

Chanut, Jean-Marie, Jean Heffer, Jacques Mairesse and Gilles Postel-Vinay (2000), L'industrie française au milieu du 19e siècle. Les enquêtes de la Statistique Générale de la France, EHESS, Paris.

Conley, Timothy G. (1999), 'GMM estimation with cross sectional dependence', Journal of Econometrics 92(1), 1-45.

Crouzet, François (2003), 'The historiography of French economic growth in the nineteenth century', Economic History Review 62(2), 215242.

Darnton, Robert (1973), 'The Encyclopédie wars of prerevolutionary France', American Historical Review 78(5), 1331-1352.

Daudin, Guillaume (2010), 'Domestic trade and market size in late eighteenth-century France', Journal of Economic History 70(3), 716-743.

de la Croix, David, Matthias Doepke and Joel Mokyr (2016), Clans, guilds, and markets: apprenticeship institutions and growth in the pre-industrial economy, Working paper.

Dickinson, Henry Winram (1939), A short history of the steam engine, Cambridge University Press, Cambridge, UK.

Diebolt, Claude, Magali Jaoul and Gilles San Martino (2005), 'Le mythe de Ferry: une analyse cliométrique', Revue d'économie politique 115(4), 471-497.

Doepke, Matthias (2004), 'Accounting for fertility decline during the transition to growth', Journal of Economic Growth 9(3), 347-383.

Fernihough, Alan and Kevin H. O'Rourke (2014), Coal and the European industrial revolution, Working Paper 19802, NBER.

Forest de Bélidor, Bernard (1737), Architecture hydraulique, ou l'art de conduire, d'élever et de ménager les eaux pour les différens besoins de la vie, Libraire de l'Artillerie et du Génie, Paris, France.

Franck, Raphaël (2010), 'Economic growth and the separation of Church and State: the French case', Economic Inquiry 48(4), 841-859. 
Franck, Raphaël (2016), 'The political consequences of income shocks: explaining the consolidation of democracy in France', Review of Economics and Statistics 98(1), 57-82.

Franck, Raphaël and Noel D. Johnson (2016), 'Can public policies lower religiosity? Evidence from school choice in France, 1878-1902', Economic History Review 69(3), 915-944.

Furet, François and Jacques Ozouf (1977), Lire et écrire. L'alphabétisation des Français de Calvin à Jules Ferry, Editions de Minuit, Paris, France.

Galor, Oded (2011), Unified Growth Theory, Princeton University Press, Princeton, NJ.

Galor, Oded and Andrew Mountford (2008), 'Trading population for productivity: Theory and evidence', Review of Economic Studies 75(4), 1143-1179.

Galor, Oded and David N. Weil (2000), 'Population, technology, and growth: From Malthusian stagnation to the demographic transition and beyond', American Economic Review 90, 806-828.

Galor, Oded and Omer Moav (2002), 'Natural selection and the origin of economic growth', The Quarterly Journal of Economics 117(4), 1133-1191.

Godechot, Jacques (1951), Les institutions de la France sous la Revolution et l'Empire, Presses Universitaires de France, Paris, France.

Goldin, Claudia and Lawrence F. Katz (1998), 'The origins of technology-skill complementary', Quarterly Journal of Economics 113(3), 693-732.

Grew, Raymond and Patrick J. Harrigan (1991), School, State, and Society: The Growth of Elementary Schooling in Nineteenth-Century France-A Quantitative Analysis, University of Michigan Press, Ann Arbor, MI.

Kanefsky, John and John Robey (1980), 'Steam engines in 18th-century Britain: a quantitative assessment', Technology and Culture 21(2), 161-186.

Labrousse, Ernest, Ruggiero Romano and François-Georges Dreyfus (1970), Le prix du froment en France au temps de la monnaie stable (1726-1913), Ecole Pratique des Hautes Etudes S.E.V.P.E.N., Paris, France.

Lagerlöf, Nils-Petter (2003), 'From Malthus to modern growth: can epidemics explain the three regimes?', International Economic Review 44(2), 755-777.

Lagerlöf, Nils-Petter (2006), 'The Galor-Weil model revisited: a quantitative exercise', Review of Economic Dynamics 9(1), 116-142.

Léon, Pierre (1976), L'affermissement du phénomène d'industrialisation, in F.Braudel and E.Labrousse, eds, 'Histoire économique et sociale de la France, vol. 3', PUF, Paris, pp. 475-616.

Lepetit, Bernard (1994), The Pre-Industrial Urban System: France, 1740-1840, Cambridge University Press, Cambridge, UK.

Lévy-Leboyer, Maurice and François Bourguignon (1990), The French Economy in the Nineteenth Century, Cambridge University Press, Cambridge, UK.

Lord, John (1923), Capital and steam power, 1750-1800, P.S. King, London, UK.

Marchand, Philippe (2005), 'L'enseignement technique et professionnel en France 1800-1919', Techniques $\&$ Culture 45(1), 3-4.

Mayeur, Françoise (1979), L'éducation des filles en France au XIXe siècle, Hachette, Paris.

Mayeur, Françoise (2003), Histoire générale de l'enseignement et de l'éducation en France: De la Révolution à l'Ecole Républicaine (1789-1930), Perrin - Collection Tempus, Paris, France.

Ministère De l'Instruction Publique, France (1865), Statistique de l'instruction primaire pour l'année 1863, Imprimerie Impériale, Paris, France. 
Mokyr, Joel (1990), The Lever of Riches, Oxford University Press, Oxford, U.K.

Mokyr, Joel (1993), The new economic history and the industrial revolution, in J.Mokyr, ed., 'The British Industrial Revolution: An Economic Perspective', Westview Press, Boulder, CO, p. 1131.

Nuvolari, Alessandro, Bart Verspagen and Nick von Tunzelmann (2011), 'Steam engines in 18th-century Britain: a quantitative assessment', Cliometrica 21(5), 291-321.

Özak, Omer (2013), Distance to the technological frontier and economic development. Southern Methodist University Working Paper.

Ramankutty, Navin, Jonathan A. Foley, John Norman and Kevin McSweeney (2002), 'The global distribution of cultivable lands: current patterns and sensitivity to possible climate change', Global Ecology and Biogeography 11(5), 377392.

Rosenberg, Nathan and Manuel Trajtenberg (2004), 'A general purpose technology at work: the Corliss steam engine in the late nineteenth-century United States', Journal of Economic History 64(1), 1-39.

Rouche, Michel (2003), Histoire générale de l'enseignement et de l'éducation en France: des origines à la renaissance (Ve siècle av. J.-C.- XVe siècle), Perrin - Collection Tempus, Paris, France.

Sée, Henri (1925), La vie économique de la France sous la Monarchie Censitaire (1815-1848), Librairie Félix Alcan, Paris.

Squicciarini, Mara P. and Nico Voigtländer (2015), 'Human capital and industrialization: evidence from the age of enlightenment', Quarterly Journal of Economics 130(4), 1-40.

Tackett, Timothy (1986), Religion, revolution, and regional culture in eighteenth-century France: the ecclesiastical oath of 1791, Princeton University Press, Princeton, NJ.

Weber, Eugen (1976), Peasants into Frenchmen: the Modernization of Rural France, 1870-1914, Stanford University Press, Stanford, CA.

Woronoff, Denis (1997), Les forges, 1811, in G.Béaur and P.Minard, eds, 'Atlas de la révolution française: Economie, Vol. 10', Editions de l'école des hautes études en sciences sociales, Paris, pp. 99-100. 
Supplementary Appendix for Online Publication

Appendix A. Descriptive Statistics

Appendix B. Additional Robustness Analysis 
Table A.1: Descriptive statistics

\begin{tabular}{|c|c|c|c|c|c|}
\hline \multirow{2}{*}{\multicolumn{6}{|c|}{ Dependent Variables }} \\
\hline Teachers, 1840 & & & & & \\
\hline & 85 & 742 & 347 & 187 & 1907 \\
\hline Teachers, 1863 & 85 & 1243 & 612 & 515 & 4903 \\
\hline Pupils Enrolled in Schools (per 10,000 inhabitants), 1840 & 85 & 874 & 414 & 314 & 1794 \\
\hline Pupils Enrolled in Schools (per 10,000 inhabitants), 1863 & 85 & 1179 & 301 & 641 & 1909 \\
\hline Apprentices (per 10,000 inhabitants), 1863 & 85 & 2.71 & 5.98 & 0 & 44.17 \\
\hline Share of Conscripts who could Read and Write, $1847-1856$ & 85 & 0.63 & 0.18 & 0.31 & 0.96 \\
\hline Share of Conscripts who could Read and Write, 1859-1868 & 85 & 0.74 & 0.15 & 0.41 & 0.98 \\
\hline School buildings per Commune, 1850 & 85 & 1.88 & 1.34 & 1.01 & 12.22 \\
\hline Schools buildings per Commune, 1863 & 85 & 2.19 & 2.56 & 1.07 & 24.54 \\
\hline Total Public Spending on Primary Schooling per Inhabitant, 1855-1863 (in French Francs) & 85 & 0.84 & 0.28 & 0.07 & 1.51 \\
\hline Commune Spending on Primary Schooling per Inhabitant, 1855-1863 (in French Francs) & 85 & 0.65 & 0.24 & 0.07 & 1.27 \\
\hline Department Spending on Primary Schooling per Inhabitant, 1855-1863 (in French Francs) & 85 & 0.08 & 0.05 & 0 & 0.33 \\
\hline Central State Spending on Primary Schooling per Inhabitant, 1855-1863 (in French Francs) & 85 & 0.10 & 0.17 & 0 & 0.85 \\
\hline \multicolumn{6}{|l|}{ Explanatory Variables } \\
\hline Number of Steam Engines & 85 & 29.20 & 66.14 & 0 & 565 \\
\hline Number of Steam Engines per Inhabitant in 1841 & 85 & 0.00006 & 0.00007 & 0 & 0.0005 \\
\hline Average Rainfall & 85 & 872.23 & 152.11 & 642.90 & 1289.24 \\
\hline Average Temperature & 85 & 10.57 & 1.50 & 4.60 & 13.73 \\
\hline Latitude & 85 & 46.59 & 2.12 & 42.60 & 50.49 \\
\hline Land Suitability & 85 & 0.75 & 0.18 & 0.21 & 0.98 \\
\hline Share of Carboniferous Area & 85 & 0.10 & 0.15 & 0 & 0.71 \\
\hline Maritime Department & 85 & 0.26 & 0.44 & 0 & 1 \\
\hline Border Department & 85 & 0.20 & 0.40 & 0 & 1 \\
\hline Distance to Paris & 85 & 353.73 & 179.53 & 1 & 686.79 \\
\hline Paris and Suburbs & 85 & 0.04 & 0.19 & 0 & 1 \\
\hline Grooms who Signed their Marriage License, $1786-1790$ & 79 & 0.42 & 0.24 & 0.05 & 0.92 \\
\hline University & 85 & 0.19 & 0.39 & 0 & 1 \\
\hline Urban Population in 1700 & 85 & 21.76 & 58.96 & 0 & 510 \\
\hline \multicolumn{6}{|l|}{ Instrumental Variables } \\
\hline Distance to Fresnes & 85 & 485.44 & 216.60 & 42.88 & 862.60 \\
\hline Deviation from Wheat Prices in 1835-1838 (baseline 1810-1829) & 85 & -.6022891 & .2304548 & -1.059889 & -.1498778 \\
\hline \multicolumn{6}{|l|}{ Variables for Robustness Analysis and Falsification Testw } \\
\hline \multicolumn{6}{|l|}{ Early Economic and Human Development } \\
\hline Encyclopedie subscribers & 85 & 82.84 & 146.36 & 0 & 1078 \\
\hline Fertility, 1806 & 85 & 0.41 & 0.11 & 0.25 & 0.84 \\
\hline Grooms who Signed their Marriage License, $1686-1690$ & 76 & 25.90 & 14.87 & 6.27 & 64.25 \\
\hline Grooms who Signed their Marriage License, $1816-1820$ & 78 & 50.61 & 22.14 & 13.35 & 96.28 \\
\hline Population Density, 1801 & 84 & 0.75 & 1.55 & 0.19 & 13.17 \\
\hline Population Density, 1821 & 85 & 0.85 & 1.94 & 0.21 & 17.15 \\
\hline Iron Forges, 1789 & 85 & 2.25 & 8.95 & 0 & 52 \\
\hline Iron Forges, 1811 & 85 & 2.65 & 11.34 & 0 & 79 \\
\hline Presence of Iron Forges, 1789 & 85 & 0.11 & 0.31 & 0 & 1 \\
\hline Presence of Iron Forges, 1811 & 85 & 0.11 & 0.31 & 0 & 1 \\
\hline Market Integration during the French Revolution & 84 & 134.68 & 107.44 & 21 & 732 \\
\hline \multicolumn{6}{|l|}{ Railroad Connection to Paris, 1860} \\
\hline Share of department's territory covered by mines, 1837 & 85 & 0.008 & 0.015 & 0.00009 & 0.086 \\
\hline \multicolumn{6}{|l|}{ Encyclopedie Wages, 1839-1847 } \\
\hline Average Male Wage, 1839-1847 & 85 & 194.40 & 34.02 & 145.60 & 342.57 \\
\hline Average Female Wage, 1839-1847 & 85 & 90.88 & 18.14 & 62.5 & 155.56 \\
\hline Averagel Child Wage, 1839-1847 & 85 & 64.44 & 13.81 & 40.96 & 111.27 \\
\hline \multicolumn{6}{|l|}{ Distance Variables } \\
\hline Distance from London & 85 & 620.71 & 219.31 & 242.93 & 1015.33 \\
\hline Distance from Marseille & 85 & 483.10 & 215.25 & 1 & 902.67 \\
\hline Distance from Lyon & 85 & 334.58 & 149.94 & 1 & 723.27 \\
\hline Distance from Rouen & 85 & 405.74 & 199.16 & 1 & 774.94 \\
\hline Distance from Mulhouse & 85 & 456.25 & 187.92 & 36.61 & 853.41 \\
\hline Distance from Bordeaux & 85 & 398.27 & 171.53 & 1 & 759.49 \\
\hline Distance from Fresnes (weeks of travel) & 85 & .471 & 0.185 & 0.045 & 0.862 \\
\hline Distance from Paris (weeks of travel) & 85 & 0.389 & 0.168 & 0.003 & 0.693 \\
\hline Distance from Marseille (weeks of travel) & 85 & 0.509 & 0.232 & 0.041 & 0.999 \\
\hline Distance from Lyon (weeks of travel) & 85 & 0.427 & .229 & 0.029 & 0.960 \\
\hline Distance from Mulhouse (weeks of travel) & 85 & 0.641 & 0.292 & 0.012 & 1.126 \\
\hline Distance from Rouen (weeks of travel) & 85 & 0.442 & 0.201 & 0.022 & 0.839 \\
\hline Distance from Bordeaux (weeks of travel) & 85 & 0.513 & 0.265 & 0.002 & 1.125 \\
\hline \multicolumn{6}{|l|}{ Wheat Prices } \\
\hline Deviation from Wheat Prices in 1835-1838 (baseline 1820-1829) & 85 & -0.082 & 0.506 & -0.931 & 1.186 \\
\hline Deviation from Wheat Prices in 1835-1838 (baseline 1815-1834) & 85 & -0.482 & 0.265 & -0.963 & 0.087 \\
\hline Deviation from Wheat Prices in 1835-1838 (baseline 1825-1834) & 85 & -0.406 & 0.472 & -1.105 & 0.885 \\
\hline Deviation from Wheat Prices in 1831-1834 (baseline 1810-1829) & 85 & -0.384 & 0.191 & -1.037 & 0.040 \\
\hline Deviation from Wheat Prices in $1839-1842$ (baseline $1810-1829$ ) & 85 & 0.067 & 0.762 & -0.765 & 5.223 \\
\hline Deviation from Wheat Prices in 1843-1846 (baseline 1810-1829) & 85 & 0.044 & 0.263 & -0.755 & 0.433 \\
\hline
\end{tabular}


Table A.2: Steam engines and workers per industrial sector: descriptive statistics

\begin{tabular}{|c|c|c|c|c|c|}
\hline & Obs. & Mean & Std. Dev. & Min. & Max. \\
\hline \multicolumn{6}{|l|}{ Steam Engines per Sector } \\
\hline Ceramics & 85 & 0.53 & 2.95 & 0 & 25 \\
\hline Chemistry & 85 & 0.84 & 3.30 & 0 & 18 \\
\hline Clothing & 85 & 0.15 & 0.81 & 0 & 6 \\
\hline Construction & 85 & 0.07 & 0.40 & 0 & 3 \\
\hline Food & 85 & 4.33 & 20.68 & 0 & 182 \\
\hline Furniture & 85 & 0 & 0 & 0 & 0 \\
\hline Leather & 85 & 0.04 & 0.24 & 0 & 2 \\
\hline Lighting & 85 & 0.06 & 0.45 & 0 & 4 \\
\hline Luxury Goods & 85 & 0.02 & 0.22 & 0 & 2 \\
\hline Metal objects & 85 & 1.51 & 5.07 & 0 & 32 \\
\hline Metallurgy & 85 & 1.79 & 5.87 & 0 & 42 \\
\hline Mines & 85 & 2.68 & 11.53 & 0 & 81 \\
\hline Sciences \& Arts & 85 & 0.22 & 1.13 & 0 & 9 \\
\hline Textile & 85 & 9.94 & 30.52 & 0 & 223 \\
\hline Transportation & 85 & 0.31 & 2.09 & 0 & 18 \\
\hline Wood & 85 & 0.26 & 1.23 & 0 & 9 \\
\hline Number of Steam Engines (total) & 85 & 29.20 & 66.14 & 0 & 565 \\
\hline \multicolumn{6}{|l|}{ Workers per Sector } \\
\hline Ceramics & 85 & 406.02 & 682.28 & 0 & 4186 \\
\hline Chemistry & 85 & 113.74 & 304.24 & 0 & 2073 \\
\hline Clothing & 85 & 320.56 & 730.68 & 0 & 4000 \\
\hline Construction & 85 & 251.54 & 309.22 & 0 & 1302 \\
\hline Food & 85 & 1781.06 & 2445.28 & 0 & 15461 \\
\hline Furniture & 85 & 37.35 & 221.92 & 0 & 2000 \\
\hline Leather & 85 & 105.45 & 137.88 & 0 & 716 \\
\hline Lighting & 85 & 18.67 & 39.81 & 0 & 215 \\
\hline Luxury Goods & 85 & 16.04 & 69.18 & 0 & 576 \\
\hline Metal objects & 85 & 815.78 & 2204.08 & 0 & 14382 \\
\hline Metallurgy & 85 & 607.16 & 867.90 & 0 & 4119 \\
\hline Mines & 85 & 788.15 & 1441.53 & 0 & 10580 \\
\hline Sciences \& Arts & 85 & 309.58 & 406.33 & 0 & 2140 \\
\hline Textile & 85 & 8132.78 & 18793.75 & 0 & 128780 \\
\hline Transportation & 85 & 98.98 & 344.48 & 0 & 2791 \\
\hline Wood & 85 & 47.85 & 127.78 & 0 & 812 \\
\hline Workers (All Sectors) & 85 & 13850.71 & 21289.12 & 540 & 135825 \\
\hline
\end{tabular}


Table B.1: The geographical diffusion of the steam engine: robustness analysis

\begin{tabular}{|c|c|c|c|c|c|c|}
\hline & $\begin{array}{l}\text { (1) } \\
\text { OLS }\end{array}$ & $\begin{array}{c}(2) \\
\text { OLS }\end{array}$ & $\begin{array}{c}(3) \\
\text { OLS } \\
\text { Number of St }\end{array}$ & $\begin{array}{c}(4) \\
\text { OLS } \\
\text { eam Engines } \\
\end{array}$ & $\begin{array}{l}(5) \\
\text { OLS }\end{array}$ & $\begin{array}{c}6) \\
\text { OLS }\end{array}$ \\
\hline Distance to Fresnes & $\begin{array}{c}-0.00684^{* * *} \\
{[0.00250]}\end{array}$ & $\begin{array}{c}-0.00682^{* * *} \\
{[0.00249]}\end{array}$ & $\begin{array}{c}-0.00625^{* * *} \\
{[0.00234]}\end{array}$ & $\begin{array}{c}-0.00626^{* * *} \\
{[0.00234]}\end{array}$ & $\begin{array}{c}-0.00611^{* *} \\
{[0.00247]}\end{array}$ & $\begin{array}{c}-0.00670^{* * *} \\
{[0.00249]}\end{array}$ \\
\hline Deviation from Wheat Prices in 1835-1838 (baseline 1810-1829) & $\begin{array}{c}-1.614^{* * *} \\
{[0.488]}\end{array}$ & $\begin{array}{c}-1.602^{* * *} \\
{[0.489]}\end{array}$ & $\begin{array}{c}-1.822^{* * * *} \\
{[0.487]}\end{array}$ & $\begin{array}{c}-1.824^{* * *} \\
{[0.487]}\end{array}$ & $\begin{array}{c}-1.141^{* *} \\
{[0.494]}\end{array}$ & $\begin{array}{c}-1.477^{* * * *} \\
{[0.507]}\end{array}$ \\
\hline Average Rainfall & $\begin{array}{c}-1.510^{*} \\
{[0.801]}\end{array}$ & $\begin{array}{c}-1.493^{*} \\
{[0.794]}\end{array}$ & $\begin{array}{c}-1.683^{* *} \\
{[0.795]}\end{array}$ & $\begin{array}{c}-1.684^{* *} \\
{[0.795]}\end{array}$ & $\begin{array}{l}-1.335^{*} \\
{[0.758]}\end{array}$ & $\begin{array}{c}-1.404^{*} \\
{[0.788]}\end{array}$ \\
\hline Average Temperature & $\begin{array}{c}1.604 \\
{[1.199]}\end{array}$ & $\begin{array}{c}1.580 \\
{[1.186]}\end{array}$ & $\begin{array}{c}1.694 \\
{[1.127]}\end{array}$ & $\begin{array}{c}1.698 \\
{[1.128]}\end{array}$ & $\begin{array}{c}1.550 \\
{[1.146]}\end{array}$ & $\begin{array}{l}1.562 \\
{[1.139]}\end{array}$ \\
\hline Latitude & $\begin{array}{l}-10.32 \\
{[8.453]}\end{array}$ & $\begin{array}{l}-10.45 \\
{[8.405]}\end{array}$ & $\begin{array}{l}-7.684 \\
{[8.251]}\end{array}$ & $\begin{array}{l}-7.663 \\
{[8.258]}\end{array}$ & $\begin{array}{l}-8.699 \\
{[8.513]}\end{array}$ & $\begin{array}{l}-9.657 \\
{[7.973]}\end{array}$ \\
\hline Land Suitability & $\begin{array}{l}-0.577 \\
{[0.395]}\end{array}$ & $\begin{array}{l}-0.577 \\
{[0.392]}\end{array}$ & $\begin{array}{l}-0.621 \\
{[0.384]}\end{array}$ & $\begin{array}{l}-0.624 \\
{[0.385]}\end{array}$ & $\begin{array}{l}-0.594^{*} \\
{[0.334]}\end{array}$ & $\begin{array}{l}-0.616 \\
{[0.390]}\end{array}$ \\
\hline Share of Carboniferous Area & $\begin{array}{l}0.0979 \\
{[0.696]}\end{array}$ & $\begin{array}{l}0.0864 \\
{[0.699]}\end{array}$ & $\begin{array}{c}-0.0543 \\
{[0.699]}\end{array}$ & $\begin{array}{l}-0.0577 \\
{[0.698]}\end{array}$ & $\begin{array}{c}0.576 \\
{[0.730]}\end{array}$ & $\begin{array}{l}-0.0709 \\
{[0.749]}\end{array}$ \\
\hline Maritime Department & $\begin{array}{c}0.538 \\
{[0.324]}\end{array}$ & $\begin{array}{l}0.544^{*} \\
{[0.315]}\end{array}$ & $\begin{array}{l}0.521^{*} \\
{[0.312]}\end{array}$ & $\begin{array}{c}0.521 \\
{[0.312]}\end{array}$ & $\begin{array}{c}0.410 \\
{[0.301]}\end{array}$ & $\begin{array}{l}0.529^{*} \\
{[0.314]}\end{array}$ \\
\hline Border Department & $\begin{array}{c}0.178 \\
{[0.337]}\end{array}$ & $\begin{array}{c}0.178 \\
{[0.334]}\end{array}$ & $\begin{array}{c}0.233 \\
{[0.330]}\end{array}$ & $\begin{array}{c}0.232 \\
{[0.329]}\end{array}$ & $\begin{array}{c}0.327 \\
{[0.318]}\end{array}$ & $\begin{array}{c}0.152 \\
{[0.333]}\end{array}$ \\
\hline Paris and Suburbs & $\begin{array}{c}-0.00732 \\
{[0.775]}\end{array}$ & $\begin{array}{c}-0.0413 \\
{[0.773]}\end{array}$ & $\begin{array}{c}0.111 \\
{[0.614]}\end{array}$ & $\begin{array}{c}0.113 \\
{[0.613]}\end{array}$ & $\begin{array}{c}0.345 \\
{[0.506]}\end{array}$ & $\begin{array}{c}-0.0106 \\
{[0.577]}\end{array}$ \\
\hline Distance to Paris & $\begin{array}{c}0.00364 \\
{[0.00234]}\end{array}$ & $\begin{array}{c}0.00358 \\
{[0.00233]}\end{array}$ & $\begin{array}{l}0.00366^{*} \\
{[0.00216]}\end{array}$ & $\begin{array}{l}0.00368^{*} \\
{[0.00216]}\end{array}$ & $\begin{array}{c}0.00336 \\
{[0.00219]}\end{array}$ & $\begin{array}{c}0.00347 \\
{[0.00232]}\end{array}$ \\
\hline Population Density, 1801 & $\begin{array}{c}0.122 \\
{[0.543]}\end{array}$ & & & & & \\
\hline Population Density, 1821 & & $\begin{array}{l}0.156 \\
{[0.496]}\end{array}$ & & & & \\
\hline Iron Forges, 1789 & & & $\begin{array}{c}0.203 \\
{[0.264]}\end{array}$ & & & \\
\hline Presence of Iron Forges, 1789 & & & $\begin{array}{c}-0.00610 \\
{[0.731]}\end{array}$ & & & \\
\hline Iron Forges, 1811 & & & & $\begin{array}{c}0.197 \\
{[0.235]}\end{array}$ & & \\
\hline Presence of Iron Forges, 1811 & & & & $\begin{array}{c}-0.00130 \\
{[0.682]}\end{array}$ & & \\
\hline Market Integration during the French Revolution & & & & & $\begin{array}{c}0.446^{* * *} \\
{[0.150]}\end{array}$ & \\
\hline Encyclopedie subscribers & & & & & & $\begin{array}{c}0.000833 \\
{[0.000892]}\end{array}$ \\
\hline Adjusted R2 & 0.554 & 0.556 & 0.567 & 0.567 & 0.593 & 0.563 \\
\hline Observations & 84 & 85 & 85 & 85 & 84 & 85 \\
\hline
\end{tabular}

Note: The dependent variable and the explanatory variables except the dummies are in logarithm. The aerial distances are measured in kilometers. Robust standard errors are reported in brackets. *** indicates significance at the $1 \%$-level, $* *$ indicates significance at the $5 \%$-level, $*$ indicates significance at the $10 \%$-level. 
Table B.2: Industrialization and human capital formation: reduced form regressions

\begin{tabular}{|c|c|c|c|c|c|c|}
\hline & (1) & (2) & (3) & $\begin{array}{c}(4) \\
\text { Reduced Form }\end{array}$ & (5) & (6) \\
\hline & $\begin{array}{c}\text { Teachers } \\
1840\end{array}$ & $\begin{array}{c}\text { Teachers } \\
1863 \\
\end{array}$ & $\begin{array}{l}\text { Pupils per } 10,000 \\
\text { inhabitants } 1840\end{array}$ & $\begin{array}{l}\text { Pupils per } 10,000 \\
\text { inhabitants } 1863 \\
\end{array}$ & $\begin{array}{c}\text { Apprentices per } 10,000 \\
\text { inhabitants } 1863\end{array}$ & $\begin{array}{l}\text { Share of Conscripts who could } \\
\text { Read and Write, 1859-1868 }\end{array}$ \\
\hline \multirow[t]{2}{*}{ Distance to Fresnes } & $-0.00288^{* * *}$ & $-0.00239^{* * *}$ & $-0.00285^{* * *}$ & $-0.00137^{* * *}$ & $-0.00702^{* * *}$ & $-0.00105^{* *}$ \\
\hline & {$[0.000844]$} & {$[0.000703]$} & {$[0.000839]$} & {$[0.000349]$} & {$[0.00230]$} & {$[0.000475]$} \\
\hline \multirow[t]{2}{*}{ Deviation from Wheat Prices in 1835-1838 (baseline 1810-1829) } & $-0.433^{*}$ & $-0.375^{* *}$ & $-0.310^{*}$ & -0.0811 & -0.432 & -0.150 \\
\hline & {$[0.230]$} & {$[0.143]$} & {$[0.178]$} & {$[0.102]$} & {$[0.462]$} & {$[0.0902]$} \\
\hline \multirow[t]{2}{*}{ noengine 3947} & -0.152 & $-0.333^{* *}$ & -0.104 & -0.0796 & -0.187 & -0.00759 \\
\hline & {$[0.168]$} & {$[0.133]$} & {$[0.139]$} & {$[0.0606]$} & {$[0.219]$} & {$[0.0911]$} \\
\hline \multirow[t]{2}{*}{ Average Rainfall } & $0.765^{* * *}$ & $0.427^{*}$ & $0.588^{* *}$ & $0.416^{* * *}$ & 0.192 & 0.149 \\
\hline & {$[0.320]$} & {$[0.247]$} & {$[0.258]$} & {$[0.121]$} & {$[0.698]$} & {$[0.119]$} \\
\hline \multirow[t]{2}{*}{ Average Temperature } & -0.501 & 0.104 & $-1.247^{* * *}$ & $-0.821^{* * * *}$ & $2.027^{* * *}$ & -0.289 \\
\hline & {$[0.435]$} & {$[0.422]$} & {$[0.332]$} & {$[0.175]$} & {$[0.936]$} & {$[0.175]$} \\
\hline \multirow[t]{2}{*}{ Latitude } & -1.649 & -0.100 & -3.819 & $-3.100^{* *}$ & $-14.48^{* *}$ & -1.862 \\
\hline & {$[3.561]$} & [2.559] & {$[2.858]$} & {$[1.461]$} & {$[5.714]$} & {$[1.764]$} \\
\hline \multirow[t]{2}{*}{ Land Suitability } & 0.135 & 0.0184 & $0.242^{*}$ & $0.140^{*}$ & -0.290 & $0.136^{* *}$ \\
\hline & {$[0.199]$} & {$[0.201]$} & {$[0.136]$} & {$[0.0721]$} & {$[0.498]$} & {$[0.0680]$} \\
\hline \multirow[t]{2}{*}{ Share of Carboniferous Area } & $-0.623^{* * *}$ & -0.107 & $-0.853^{* * *}$ & $-0.444^{* * * *}$ & -0.310 & $-0.478^{* *}$ \\
\hline & {$[0.293]$} & {$[0.256]$} & {$[0.276]$} & {$[0.161]$} & {$[0.597]$} & {$[0.203]$} \\
\hline \multirow[t]{2}{*}{ Maritime Department } & 0.181 & $0.171^{*}$ & -0.0324 & 0.0286 & 0.427 & -0.0672 \\
\hline & {$[0.121]$} & {$[0.0915]$} & {$[0.0855]$} & {$[0.0429]$} & {$[0.282]$} & {$[0.0480]$} \\
\hline \multirow[t]{2}{*}{ Border Department } & & & & 0.00338 & $0.776^{* *}$ & 0.0316 \\
\hline & {$[0.145]$} & {$[0.123]$} & {$[0.106]$} & {$[0.0530]$} & {$[0.343]$} & {$[0.0536]$} \\
\hline \multirow[t]{2}{*}{ Distance to Paris } & $0.00258^{* * *}$ & $0.00228^{* * *}$ & $0.00205^{* * * *}$ & 0.000543 & 0.00268 & $0.000697^{*}$ \\
\hline & {$[0.000869]$} & {$[0.000665]$} & {$[0.000770]$} & {$[0.000370]$} & {$[0.00241]$} & {$[0.000416]$} \\
\hline \multirow[t]{2}{*}{ Paris and Suburbs } & $0.594^{* *}$ & $0.742^{*}$ & 0.180 & -0.0103 & $0.929^{*}$ & $0.225^{* * *}$ \\
\hline & {$[0.264]$} & {$[0.401]$} & {$[0.203]$} & {$[0.147]$} & {$[0.551]$} & {$[0.0587]$} \\
\hline Observations & 85 & 85 & 85 & 85 & 85 & 85 \\
\hline
\end{tabular}

Note: The explanatory variables except the dummies are in logarithm. The aerial distances are measured in kilometers. Robust standard errors are reported in brackets. ${ }^{* * *}$ indicates significance at the $1 \%$-level, $* *$ indicates significance at the $5 \%$-level, $*$ indicates significance at the $10 \%-l e v e l$. 
Table B.3: Industrialization and human capital formation, accounting for the distance between London and each department

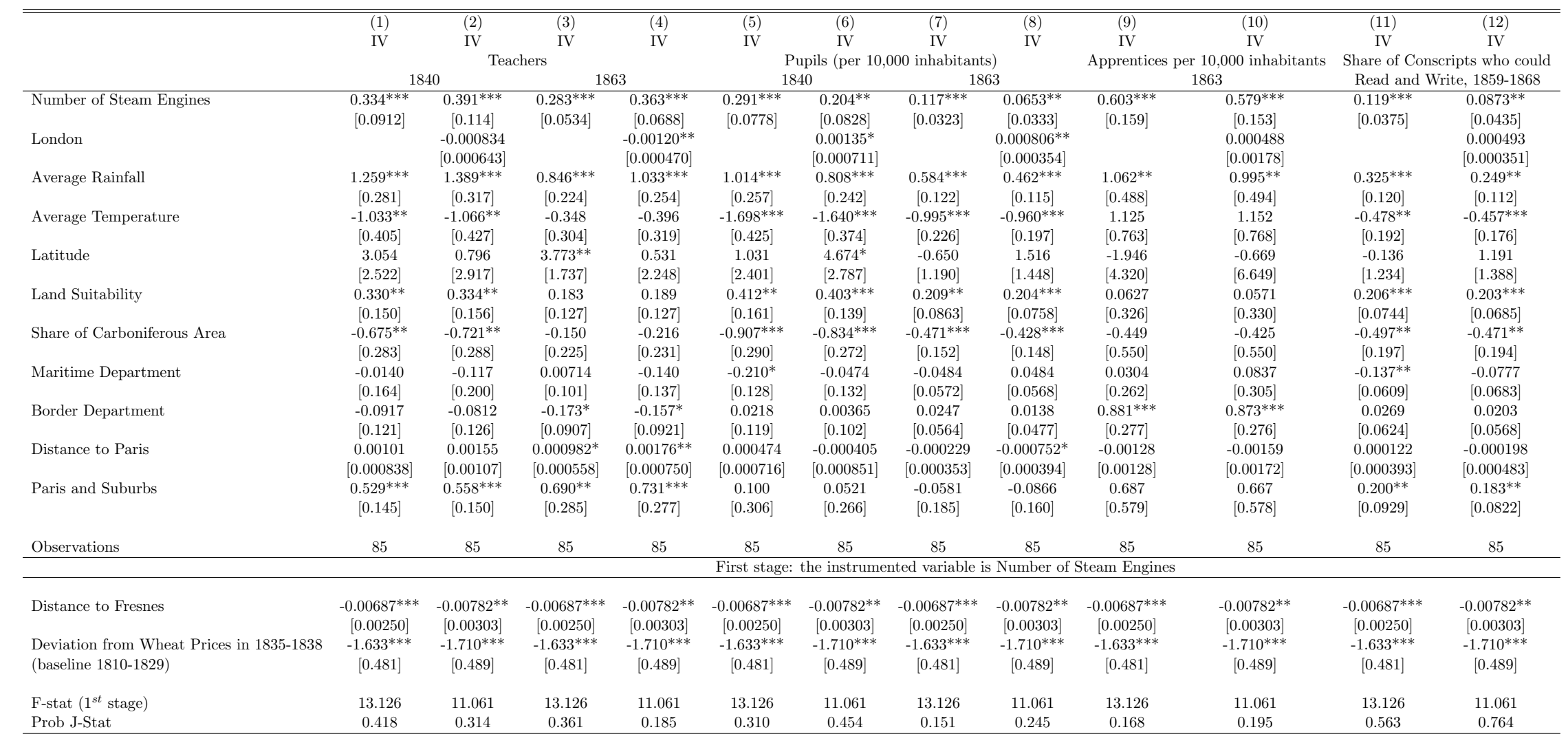

Note: Robust standard errors are reported in brackets. ${ }^{* *}$ indicates significance at the $1 \%$-level, ${ }^{* *}$ indicates significance t the $5 \%$-level, $*$ indicates significance at the $10 \%$-level. 
Table B.4: Industrialization and human capital formation, accounting for grooms who could sign their marriage license in 1686-1690 and 1816-1820

\begin{tabular}{|c|c|c|c|c|c|c|c|c|c|c|c|c|c|c|c|c|c|c|}
\hline & \multirow[t]{2}{*}{$\begin{array}{l}(1) \\
\text { IV }\end{array}$} & \multirow{2}{*}{$\begin{array}{c}(2) \\
\text { IV } \\
\text { Teachers } \\
1840 \\
\end{array}$} & \multirow[t]{2}{*}{$\begin{array}{l}(3) \\
\text { IV }\end{array}$} & \multirow[t]{2}{*}{$\begin{array}{l}(4) \\
\text { IV }\end{array}$} & \multirow{2}{*}{$\begin{array}{c}\text { (5) } \\
\text { IV } \\
\text { Teachers } \\
1863 \\
\end{array}$} & \multirow[t]{2}{*}{$\begin{array}{l}(6) \\
\text { IV }\end{array}$} & $\begin{array}{l}(7) \\
\text { IV }\end{array}$ & $\begin{array}{l}(8) \\
\text { IV }\end{array}$ & \multirow{2}{*}{$\begin{array}{c}(9) \\
\text { IV } \\
\text { Ibitants) }\end{array}$} & $\begin{array}{l}\text { (10) } \\
\text { IV }\end{array}$ & $\begin{array}{l}(11) \\
\text { IV }\end{array}$ & \multirow{2}{*}{ 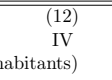 } & \multirow{2}{*}{$\begin{array}{l}\text { (13) } \\
\text { IV } \\
\text { Apprentice }\end{array}$} & \multirow{2}{*}{\multicolumn{2}{|c|}{$\begin{array}{cc}(14) & \text { (15) } \\
\text { IV } & \text { IV } \\
\text { s per } 10,000 & \text { inhabitants }\end{array}$}} & & & \\
\hline & & & & & & & Pupils (pe & $\begin{array}{c}\text { er } 10,000 \mathrm{inh} \\
1840\end{array}$ & & Pupils (pe & $\begin{array}{c}\text { er } 10,000 \mathrm{inh} \\
1863\end{array}$ & & & & & \multicolumn{2}{|c|}{$\begin{array}{l}\text { IV IV IV } \\
\text { Share of Conscripts who could }\end{array}$} & \\
\hline Number of Steam Engines & $0.334^{* * *}$ & $0.358^{* * * *}$ & $0.274^{* * * *}$ & $0.283^{* * *}$ & $0.311 * * *$ & $0.275^{* * *}$ & $0.291^{* * *}$ & $0.303 * * *$ & $0.222^{* * *}$ & $0.117^{* * *}$ & $0.131^{* * *}$ & $0.0941^{* * *}$ & $0.603^{* * *}$ & $0.637^{* * *}$ & $0.629 * * *$ & $0.119^{* * *}$ & $0.108^{* * *}$ & $0.0709^{* * * *}$ \\
\hline & {$[0.0912]$} & {$[0.0947]$} & {$[0.0894]$} & {$[0.0534]$} & {$[0.0583]$} & {$[0.0598]$} & {$[0.0778]$} & {$[0.0721]$} & {$[0.0579]$} & {$[0.0323]$} & {$[0.0318]$} & {$[0.0309]$} & {$[0.159]$} & {$[0.171]$} & {$[0.181]$} & {$[0.0375]$} & {$[0.0310]$} & {$[0.0258]$} \\
\hline Grooms who Signed their Marriage License, $1686-1690$ & & $\begin{array}{c}0.919^{* * *} \\
{[0.351]}\end{array}$ & & & $\begin{array}{l}0.139 \\
{[0.292]}\end{array}$ & & & $\begin{array}{c}1.257^{* * *} \\
{[0.341]}\end{array}$ & & & $\begin{array}{l}0.304^{*} \\
{[0.168]}\end{array}$ & & & $\begin{array}{l}-0.299 \\
{[0.839]}\end{array}$ & & & $\begin{array}{c}0.821^{* * * *} \\
{[0.149]}\end{array}$ & \\
\hline Grooms who Signed their Marriage License, $1816-1820$ & & & $1.127^{* * *}$ & & & 0.307 & & & $1.562^{* * *}$ & & & $0.503^{* * *}$ & & & -0.508 & & & $0.830^{* * *}$ \\
\hline Average Rainfall & $1.259^{* * *}$ & $1.421^{* * *}$ & {$\left[0.276^{* *}\right.$} & $0.846^{* * *}$ & $0.850^{* * *}$ & $\begin{array}{l}0.218 \mathrm{I} \\
0.702^{* *}\end{array}$ & $1.014^{* * *}$ & $1.255^{* * *}$ & {$\left[\begin{array}{l}{[0.234]} \\
0.428\end{array}\right.$} & $0.584^{* * *}$ & $0.678^{* * *}$ & {$[0.127]$} & $1.062^{* *}$ & $1.230^{* *}$ & $\begin{array}{l}{[0.633]} \\
1.381^{* *}\end{array}$ & $0.325^{* * *}$ & $0.426^{* * *}$ & $\begin{array}{l}{[0.113]} \\
-0.0364\end{array}$ \\
\hline & {$[0.281]$} & {$[0.303]$} & {$[0.340]$} & {$[0.224]$} & {$[0.246]$} & {$[0.285]$} & {$[0.257]$} & {$[0.287]$} & {$[0.326]$} & {$[0.122]$} & {$[0.129]$} & {$[0.150]$} & & {$[0.541]$} & {$[0.665]$} & {$[0.120]$} & {$[0.135]$} & $\begin{array}{l}-0.0364 \\
{[0.158]}\end{array}$ \\
\hline Average Temperature & $-1.033^{* *}$ & -0.0844 & -0.213 & -0.348 & -0.125 & -0.236 & $-1.698 * * *$ & -0.415 & -0.519 & $-0.995^{* * * *}$ & $-0.508^{* *}$ & $-0.604^{* * * *}$ & 1.125 & 1.538 & 0.829 & $-0.478^{* *}$ & 0.0801 & 0.101 \\
\hline & {$[0.405]$} & {$[0.484]$} & {$[0.443]$} & {$[0.304]$} & {$[0.372]$} & {$[0.353]$} & {$[0.425]$} & {$[0.372]$} & {$[0.365]$} & {$[0.226]$} & {$[0.204]$} & {$[0.211]$} & {$[0.763]$} & {$[0.998]$} & {$[1.019]$} & {$[0.192]$} & {$[0.204]$} & {$[0.155]$} \\
\hline Latitude & 3.054 & 4.135 & 1.304 & $3.773^{* *}$ & $4.065^{* *}$ & $3.017^{*}$ & 1.031 & 1.734 & -2.238 & -0.650 & -0.639 & $-1.991^{*}$ & -1.9 & 0.627 & -0.9 & -0.136 & -0.140 & $-1.783^{* *}$ \\
\hline & {$[2.522]$} & {$[2.655]$} & & {$[1.737]$} & {$[1.922]$} & {$[1.79$} & {$[2.401]$} & {$[2.422]$} & {$[1.983]$} & {$[1.190]$} & {$[1.120]$} & {$[1.0$} & {$[4.3$} & {$[5.327]$} & {$[5.2$} & {$[1.234]$} & {$[1.254]$} & {$[0.885]$} \\
\hline Land Suitability & $0.330^{* *}$ & $0.262^{*}$ & 0.0573 & 0.183 & 0.139 & 0.0970 & $0.412^{* *}$ & $0.319^{* *}$ & 0.00840 & $0.209^{* *}$ & $0.170^{* *}$ & 0.07 & 0.0 & 0.152 & & $0.206^{* * *}$ & $0.169^{* *}$ & -0.00324 \\
\hline & {$[0.150]$} & {$[0.145]$} & {$[0.156]$} & {$[0.127]$} & {$[0.134]$} & {$[0.146]$} & {$[0.161]$} & {$[0.148]$} & {$[0.133]$} & {$[0.0863]$} & {$[0.0793]$} & {$[0.07$} & {$[0.3$} & & {$[0$.} & {$[0.0744]$} & {$[0.0672]$} & {$[0.0587]$} \\
\hline Share of Carboniferous Area & $-0.675^{* *}$ & -0.504 & -0.2 & -0.150 & -0.0632 & 0.0797 & $-0.907^{* * * *}$ & $-0.891^{* * * *}$ & $-0.464^{*}$ & $-0.471^{* * * *}$ & $-0.568^{* * * *}$ & -0.3 & -0.4 & -0.722 & -0 . & $-0.497 * *$ & $-0.372^{*}$ & -0.239 \\
\hline & {$[0.283]$} & {$[0.326]$} & {$[0.30$} & {$[0.225]$} & {$[0.264]$} & {$[0.243]$} & {$[0.290]$} & {$[0.297]$} & {$[0.258]$} & {$[0.152]$} & {$[0.154]$} & {$[0.1$} & {$[0.5$} & & & {$[0.197]$} & {$[0.196]$} & {$[0.163]$} \\
\hline Maritime Department & -0.0140 & -0.133 & 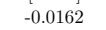 & 0.00714 & -0.0175 & 0.0301 & $-0.210^{*}$ & $-0.405^{* * * *}$ & $-0.266^{* *}$ & -0.04 & $-0.126^{* *}$ & -0.0 & 0.0304 & -0.0707 & -0.000509 & $-0.137^{* *}$ & $-0.203^{* * *}$ & $-0.142^{* * *}$ \\
\hline & {$[0.164]$} & {$[0.203]$} & {$[0.168]$} & {$[0.101]$} & {$[0.131]$} & {$[0.115]$} & {$[0.128]$} & {$[0.142]$} & {$[0.113]$} & {$[0.05$} & & {$[0.05$} & {$[0.262]$} & {$[0.328]$} & & & & {$[0.0468]$} \\
\hline Border Department & -0.0917 & -0.0533 & -0.184 & $-0.173^{*}$ & -0.119 & -0.177 & 0.0218 & 0.01 & -0.138 & 0.0 & & -0.0 & 0.88 & 0.95 & 0.955 & 0.02 & -0.00575 & -0.0654 \\
\hline & {$[0.121]$} & {$[0.111]$} & {$[0.121]$} & {$[0.0907]$} & {$[0.0867]$} & {$[0.0898]$} & {$[0.119]$} & {$[0.107]$} & {$[0.0846]$} & {$[0.0564]$} & {$[0.0560]$} & {$[0.0460]$} & {$[0.277]$} & {$[0.303]$} & {$[0.286]$} & {$[0.0624]$} & {$[0.0520]$} & {$[0.0432]$} \\
\hline Distance to Paris & 0.00101 & 0.00129 & 0.000718 & $0.000982^{*}$ & 0.00101 & 0.000855 & 0.000474 & 0.000744 & $-6.33 \mathrm{e}-05$ & $\begin{array}{r}-0.000229 \\
-0.02\end{array}$ & -0.000254 & -0.000477 & -0.00128 & -0.000856 & -0.00115 & 0.000122 & 0.000272 & -0.000138 \\
\hline & {$[0.000838]$} & [0.000931] & {$[0.000753]$} & {$[0.000558]$} & {$[0.000634]$} & {$[0.000547]$} & {$[0.000716]$} & {$[0.000740]$} & {$[0.000532]$} & {$[0.000353]$} & {$[0.000354]$} & {$[0.000300]$} & {$[0.00128]$} & {$[0.00153]$} & {$[0.00150]$} & {$[0.000393]$} & {$[0.000389]$} & {$[0.000279]$} \\
\hline Paris and Suburbs & $0.529^{* * *}$ & $0.403^{* * *}$ & 0.205 & $0.690^{* *}$ & $0.338^{* * * * *}$ & $0.285^{* *}$ & 0.100 & 0.221 & -0.0434 & -0.0581 & 0.0601 & -0.0225 & 0.687 & 0.602 & 0.669 & $0.200^{* *}$ & 0.145 & 0.00640 \\
\hline & {$[0.145]$} & {$[0.143]$} & {$[0.127]$} & {$[0.285]$} & {$[0.117]$} & {$[0.116]$} & {$[0.306]$} & {$[0.231]$} & {$[0.256]$} & {$[0.185]$} & {$[0.141]$} & {$[0.148]$} & {$[0.579]$} & {$[0.811]$} & {$[0.797]$} & {$[0.0929]$} & {$[0.0912]$} & {$[0.102]$} \\
\hline Observations & 85 & 76 & 78 & 85 & 76 & 78 & 85 & 76 & 78 & 85 & 76 & 78 & 85 & 76 & 78 & 85 & 76 & 78 \\
\hline & & & & & & & First ste & tage: the inst & rumented var & iable is Numbe & er of Steam F & Engines & & & & & & \\
\hline Distance to Fresnes & $\begin{array}{c}-0.00687 * * * \\
{[0.00250]}\end{array}$ & $\begin{array}{c}-0.00683^{* * *} \\
{[0.00262]}\end{array}$ & $\begin{array}{c}-0.00579^{* *} * \\
{[0.00260]}\end{array}$ & $\begin{array}{l}-0.00687^{* * *} \\
{[0.00250]}\end{array}$ & $\begin{array}{c}-0.00683^{* *} * \\
{[0.00262]}\end{array}$ & $\begin{array}{c}-0.00579 * * \\
{[0.00260]}\end{array}$ & $\begin{array}{c}-0.00688^{* * * *} \\
{[0.00250]}\end{array}$ & $\begin{array}{c}-0.00688^{* * *} \\
{[0.00262]}\end{array}$ & $\begin{array}{c}-0.00579 * * \\
{[0.00260]}\end{array}$ & $\begin{array}{c}-0.00687^{* * * *} \\
{[0.00250]}\end{array}$ & $\begin{array}{c}-0.00683^{* * *} \\
{[0.00262]}\end{array}$ & $\begin{array}{c}-0.00579 * * * \\
{[0.00260]}\end{array}$ & $\begin{array}{l}-0.00687 * * * \\
{[0.00250]}\end{array}$ & $\begin{array}{c}-0.00688^{*} * \\
{[0.0262]}\end{array}$ & $\begin{array}{c}-0.00579 * * \\
{[0.00260]}\end{array}$ & $\begin{array}{c}-0.00687 * * * \\
{[0.00250]}\end{array}$ & $\begin{array}{l}-0.00688^{*} * \\
{[0.0262]}\end{array}$ & $\begin{array}{c}-0.00577^{*} * \\
{[0.00260]}\end{array}$ \\
\hline $\begin{array}{l}\text { Deviation from Wheat Prices in } 1835-1838 \\
\text { (baseline 1810-1829) }\end{array}$ & $\begin{array}{c}-1.633^{* * *} \\
{[0.481]}\end{array}$ & $\begin{array}{l}-1.525 * * * * \\
{[0.520]}\end{array}$ & $\begin{array}{c}-1.611^{* * *} \\
{[0.510]}\end{array}$ & $\begin{array}{c}-1.633 * * * \\
{[0.481]}\end{array}$ & $\begin{array}{c}-1.525^{* * *} \\
{[0.520]}\end{array}$ & $\begin{array}{l}-1.611 * * * \\
{[0.510]}\end{array}$ & $\begin{array}{c}-1.633^{* * *} \\
{[0.481]}\end{array}$ & $\begin{array}{c}-1.525 * * * \\
{[0.520]}\end{array}$ & $\begin{array}{c}-1.611 * * * \\
{[0.510]}\end{array}$ & $\begin{array}{l}-1.633^{* * *} \\
{[0.481]}\end{array}$ & $\begin{array}{c}-1.525^{* * *} \\
{[0.520]}\end{array}$ & $\begin{array}{c}-1.611^{* * *} \\
{[0.510]}\end{array}$ & $\begin{array}{c}-1.633^{* * *} \\
{[0.481]}\end{array}$ & $\begin{array}{c}-1.525^{* * *} \\
{[0.520]}\end{array}$ & $\begin{array}{c}-1.611^{* * *} \\
{[0.510]}\end{array}$ & $\begin{array}{l}-1.633^{* * *} \\
{[0.481]}\end{array}$ & $\begin{array}{c}-1.525^{* * * *} \\
{[0.520]}\end{array}$ & $\begin{array}{c}-1.611^{* * * *} \\
{[0.510]}\end{array}$ \\
\hline & & & & & & & & & & 13 & & & & & & & 493 & \\
\hline Prob J-S & 0.418 & 0.707 & 0.510 & 0.361 & 0.725 & 0.517 & 0.310 & 0.466 & 0.328 & 0.151 & 0.317 & 0.220 & 0.168 & 0.218 & 0.111 & 0.563 & 0.565 & 0.665 \\
\hline
\end{tabular}

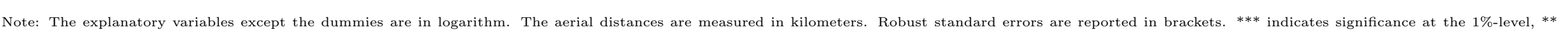

indicates significance at the $5 \%$-level, $*$ indicates significance at the $10 \%$-level. 
Table B.5: Industrialization per inhabitant in 1841 and human capital formation

\begin{tabular}{|c|c|c|c|c|c|c|c|c|c|c|c|c|}
\hline & $\begin{array}{l}\overline{~(1)} \\
\text { IV }\end{array}$ & $\begin{array}{l}\text { IV } \\
\end{array}$ & $\begin{array}{l}(3) \\
\text { IV }\end{array}$ & $\begin{array}{l}(4) \\
\text { IV }\end{array}$ & $\begin{array}{l}(5) \\
\text { IV }\end{array}$ & $\begin{array}{l}\text { IV } \\
\text { IV }\end{array}$ & $\begin{array}{ll} & (7) \\
\text { IV }\end{array}$ & $\begin{array}{l}\overline{~(8)} \\
\text { IV }\end{array}$ & \multirow{2}{*}{$\begin{array}{c}(9) \\
\text { IV } \\
\text { Apprentices }\end{array}$} & $\begin{array}{l}\text { (10) } \\
\text { IV }\end{array}$ & \multirow{3}{*}{\multicolumn{2}{|c|}{$\begin{array}{cc}(11) & (12) \\
\text { IV } & \text { IV } \\
\text { Share of Conscripts who could } & \text { Read and Write, 1859-1868 }\end{array}$}} \\
\hline & \multirow{2}{*}{\multicolumn{2}{|c|}{1840}} & \multirow{2}{*}{\multicolumn{2}{|c|}{1863}} & \multirow{2}{*}{\multicolumn{4}{|c|}{$\begin{array}{l}\text { Pupils (per 10,000 inhabitants) } \\
1840\end{array}$}} & & & & \\
\hline & & & & & & & & & \multicolumn{2}{|c|}{1863} & & \\
\hline Number of Steam Engines & $0.334^{* * *}$ & & $0.283^{* * *}$ & & $0.291 * * *$ & & $0.117^{* * *}$ & & $0.603^{* * *}$ & & $0.119^{* * *}$ & \\
\hline Numer of Steam Engines per 10,000 Inhabitants, 1841 & & $\begin{array}{c}1.058^{* * *} \\
{[0.338]}\end{array}$ & & $\begin{array}{c}0.895 * * * \\
{[0.205]}\end{array}$ & {$[0.0110]$} & $\begin{array}{c}0.924^{* * *} \\
{[0.2931}\end{array}$ & & $\begin{array}{l}0.3733^{* * *} \\
{[0.115]}\end{array}$ & {$[0.109]$} & $1.930^{* * *}$ & 0.03 & $0.377^{* * *}$ \\
\hline Average Rainfall & $1.259^{* * *}$ & $1.317^{* * * *}$ & $0.846^{* * *}$ & $0.896 * * *$ & $1.014^{* * *}$ & $1.066^{* * *}$ & $0.584^{* * *}$ & $0.600^{* * *}$ & $1.062^{* *}$ & $1.175^{* *}$ & $0.325^{* * *}$ & $0.346^{* * * *}$ \\
\hline & {$[0.281]$} & {$[0.294]$} & {$[0.224]$} & {$[0.234]$} & {$[0.257]$} & {$[0.261]$} & {$[0.122]$} & {$[0.122]$} & {$[0.488]$} & {$[0.522]$} & {$[0.120]$} & {$[0.115]$} \\
\hline Average Temperature & $-1.033^{* *}$ & $-0.972^{* *}$ & -0.348 & -0.296 & $-1.698^{* * *}$ & $-1.643^{* * * *}$ & $-0.995^{* * *}$ & $-0.972^{* * *}$ & 1.125 & $1.243^{*}$ & $-0.478^{* *}$ & $-0.456^{* *}$ \\
\hline & {$[0.405]$} & {$[0.410]$} & {$[0.304]$} & {$[0.329]$} & {$[0.425]$} & {$[0.389]$} & {$[0.226]$} & {$[0.208]$} & {$[0.763]$} & {$[0.676]$} & {$[0.192]$} & {$[0.185]$} \\
\hline Latitude & 3.054 & 4.239 & $3.773^{* *}$ & $4.776^{* *}$ & 1.031 & 2.060 & -0.650 & -0.237 & -1.946 & 0.186 & -0.136 & 0.286 \\
\hline & {$[2.522]$} & {$[2.851]$} & {$[1.737]$} & {$[2.051]$} & [2.401] & {$[2.535]$} & {$[1.190]$} & {$[1.242]$} & {$[4.320]$} & {$[4.104]$} & {$[1.234]$} & {$[1.324]$} \\
\hline Land Suitability & $0.330^{* *}$ & $0.340^{* *}$ & 0.183 & 0.191 & $0.412^{* *}$ & $0.420^{* *}$ & $0.209^{* *}$ & $0.212^{* *}$ & 0.0627 & 0.0788 & $0.206^{* * *}$ & $0.209^{* *}$ \\
\hline & {$[0.150]$} & {$[0.171]$} & {$[0.127]$} & {$[0.145]$} & {$[0.161]$} & {$[0.174]$} & {$[0.0863]$} & {$[0.0898]$} & {$[0.326]$} & {$[0.316]$} & {$[0.0744]$} & {$[0.0827]$} \\
\hline Share of Carboniferous Area & $-0.675^{* *}$ & $-0.800^{* *}$ & -0.150 & -0.255 & $-0.907^{* * *}$ & $-1.016^{* * *}$ & $-0.471^{* * *}$ & $-0.515^{* * *}$ & -0.449 & -0.677 & $-0.497^{* *}$ & $-0.542^{* * *}$ \\
\hline & {$[0.283]$} & {$[0.323]$} & {$[0.225]$} & {$[0.261]$} & {$[0.290]$} & {$[0.306]$} & {$[0.152]$} & {$[0.159]$} & {$[0.550]$} & {$[0.514]$} & {$[0.197]$} & {$[0.209]$} \\
\hline Maritime Department & -0.0140 & -0.00924 & 0.00714 & 0.0112 & $-0.210^{*}$ & -0.207 & -0.0484 & -0.04 & 0.0304 & 0.03 & $-0.137^{* *}$ & $-0.136^{* *}$ \\
\hline & {$[0.164]$} & {$[0.185]$} & {$[0.101]$} & {$[0.118]$} & {$[0.128]$} & {$[0.140]$} & {$[0.0572]$} & {$[0.0613]$} & {$[0.262]$} & {$[0.251]$} & {$[0.0609]$} & {$[0.0673]$} \\
\hline Border Department & -0.0917 & -0.119 & $-0.173^{*}$ & $-0.196^{*}$ & 0.0218 & -0.00225 & 0.0247 & 0.0148 & $0.881^{* * *}$ & $0.830^{* * *}$ & 0.0269 & 0.0171 \\
\hline & {$[0.121]$} & {$[0.146]$} & {$[0.0907]$} & {$[0.110]$} & {$[0.119]$} & {$[0.122]$} & {$[0.0564]$} & {$[0.0545]$} & {$[0.277]$} & {$[0.262]$} & {$[0.0624]$} & {$[0.0614]$} \\
\hline Distance to Paris & $\begin{array}{l}0.00101 \\
{[0.000838]}\end{array}$ & 0.00107 & $0.000982^{2}$ & 0.00103 & 0.000474 & 0.000527 & -0.000229 & $\begin{array}{l}-0.000208 \\
-0.00368\end{array}$ & $\begin{aligned}-0.00128 \\
-0.0182\end{aligned}$ & -0.00116 & 0.000122 & 0.000144 \\
\hline Paris and Suburbs & $\begin{array}{c}{\left[0.00080382^{* * *}\right]} \\
00.145]\end{array}$ & $\begin{array}{c}{[0.000950]} \\
0.677^{* * *} \\
{[0.208]}\end{array}$ & $\begin{array}{c}{[0.0005585]} \\
0.690^{* *} \\
{[0.285]}\end{array}$ & $\begin{array}{c}{[0.000647]} \\
0.815^{* *} \\
{[0.377]}\end{array}$ & $\begin{array}{c}{[0.000716]} \\
0.100 \\
{[0.306]}\end{array}$ & $\begin{array}{c}{[0.000765]} \\
0.229 \\
{[0.233]}\end{array}$ & $\begin{array}{l}{[0.000353]} \\
-0.0581 \\
{[0.185]}\end{array}$ & $\begin{array}{l}{[0.0000366]} \\
-0.00634 \\
{[0.153]}\end{array}$ & $\begin{array}{c}{[0.00128]} \\
0.687 \\
{[0.579]}\end{array}$ & $\begin{array}{c}{[0.00129]} \\
0.954 \\
{[0.634]}\end{array}$ & $\begin{array}{c}{[0.000393]} \\
0.200 * \\
{[0.0929]}\end{array}$ & $\begin{array}{c}{[0.000416]} \\
0.253^{* * *} \\
{[0.0871]}\end{array}$ \\
\hline Observations & 85 & 85 & 85 & 85 & 85 & 85 & 85 & 85 & 85 & 85 & 85 & 85 \\
\hline & & & & & First stage: & the instrume & nted variable is & Number of & Steam Engines & & & \\
\hline Distance to Fresnes & $\begin{array}{c}-0.00687^{* * *} \\
{[0.00250]}\end{array}$ & $\begin{array}{c}-0.00230^{* *} \\
{[0.00103]}\end{array}$ & $\begin{array}{c}-0.00687^{* * *} \\
{[0.00250]}\end{array}$ & $\begin{array}{c}-0.00230^{* *} \\
{[0.00103]}\end{array}$ & $\begin{array}{c}-0.00687^{* * *} \\
{[0.00250]}\end{array}$ & $\begin{array}{c}-0.00230^{* *} \\
{[0.00103]}\end{array}$ & $\begin{array}{c}-0.00687 * * * \\
{[0.00250]}\end{array}$ & $\begin{array}{c}-0.00230^{* *} \\
{[0.00103]}\end{array}$ & $\begin{array}{c}-0.00687 * * * \\
{[0.00250]}\end{array}$ & $\begin{array}{c}-0.00230^{* * *} \\
{[0.00103]}\end{array}$ & $\begin{array}{c}-0.00687 * * * * \\
{[0.00250]}\end{array}$ & $\begin{array}{c}-0.00230^{* * *} \\
{[0.00103]}\end{array}$ \\
\hline $\begin{array}{l}\text { Deviation from Wheat Prices in 1835-1838 } \\
\text { (baseline 1810-1829) }\end{array}$ & $\begin{array}{c}-1.633^{* * *} \\
{[0.481]}\end{array}$ & $\begin{array}{l}-0.495^{* * *} \\
{[0.147]}\end{array}$ & $\begin{array}{c}-1.633^{* * *} \\
{[0.481]}\end{array}$ & $\begin{array}{c}-0.495^{* * *} \\
{[0.147]}\end{array}$ & $\begin{array}{c}-1.633^{* * *} \\
{[0.481]}\end{array}$ & $\begin{array}{c}-0.495^{* * *} \\
{[0.147]}\end{array}$ & $\begin{array}{c}-1.633^{* * *} \\
{[0.481]}\end{array}$ & $\begin{array}{c}-0.495^{* * *} \\
{[0.147]}\end{array}$ & $\begin{array}{l}-1.633^{* * *} \\
{[0.481]}\end{array}$ & $\begin{array}{c}-0.495^{* * *} \\
{[0.147]}\end{array}$ & $\begin{array}{c}-1.633^{* * *} \\
{[0.481]}\end{array}$ & $\begin{array}{c}-0.495^{* * *} \\
{[0.147]}\end{array}$ \\
\hline F-stat ( $1^{s t}$ stage $)$ & 13.126 & 10.484 & 13.126 & 10.484 & 13.126 & 10.484 & 13.126 & 10.484 & 13.1 & 10.484 & 13.126 & 10.484 \\
\hline Prob J-Stat & 0.418 & 0.590 & 0.361 & 0.553 & 0.310 & 0.412 & 0.151 & 0.208 & 0.168 & 0.224 & 0.563 & 0.664 \\
\hline
\end{tabular}

Note: Robust standard errors are reported in brackets. ${ }^{* * *}$ indicates significance at the $1 \%$-level, ${ }^{* *}$ indicates significance $t$ the $5 \%$-level, ${ }^{*}$ indicates significance at the $10 \%$-level. 
Table B.6: Industrialization and human capital formation, accounting for iron forges before 1815

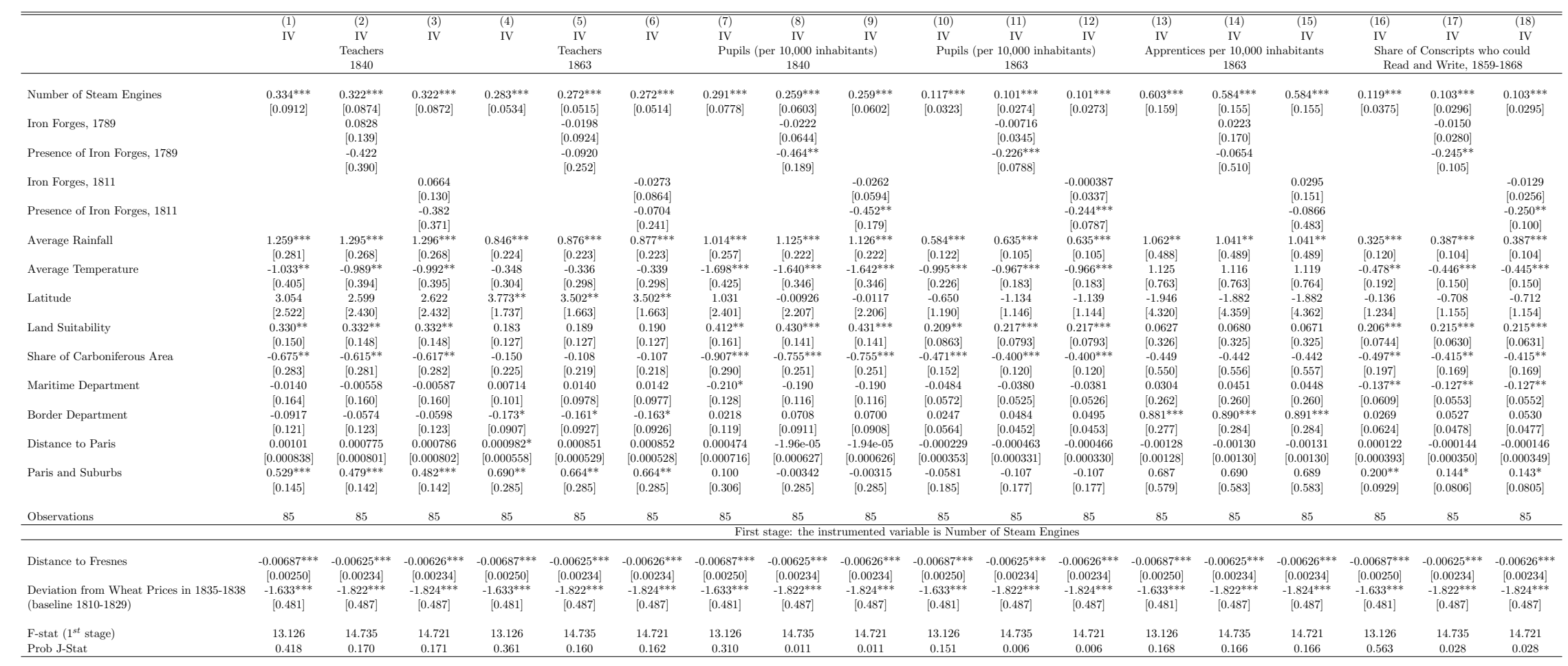

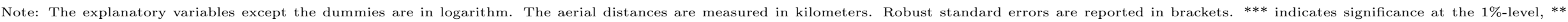
indicates significance at the $5 \%$-level, * indicates significance at the $10 \%$-level. 
Table B.7: Industrialization and human capital formation, accounting for mines in 1837

\begin{tabular}{|c|c|c|c|c|c|c|c|c|c|c|c|c|}
\hline & $\begin{array}{l}(1) \\
\text { IV }\end{array}$ & $\begin{array}{ll}(2) \\
\text { IV }\end{array}$ & $\begin{array}{l}(3) \\
\text { IV }\end{array}$ & $\begin{array}{l}(4) \\
\text { IV }\end{array}$ & $\begin{array}{l}(5) \\
\text { IV }\end{array}$ & $\begin{array}{l}(6) \\
\text { IV }\end{array}$ & $\begin{array}{l}\text { (7) } \\
\text { IV }\end{array}$ & $\begin{array}{l}(8) \\
\text { IV }\end{array}$ & $\begin{array}{l}(9) \\
\text { IV }\end{array}$ & $\begin{array}{l}\text { (10) } \\
\text { IV }\end{array}$ & $\begin{array}{c}(11) \\
\text { IV }\end{array}$ & $\begin{array}{c}(12) \\
\text { IV }\end{array}$ \\
\hline & \multirow{2}{*}{\multicolumn{4}{|c|}{ Teachers }} & \multirow{2}{*}{\multicolumn{4}{|c|}{$\begin{array}{lr}\text { Pupils (per 10,000 inhabitants) } \\
1840\end{array}$}} & \multirow{2}{*}{\multicolumn{2}{|c|}{$\begin{array}{l}\text { Apprentices per 10,000 inhabitants } \\
1863\end{array}$}} & \multirow{2}{*}{\multicolumn{2}{|c|}{$\begin{array}{l}\text { Share of Conscripts who coulc } \\
\text { Read and Write, 1859-1868 }\end{array}$}} \\
\hline & & & & & & & & & & & & \\
\hline \multirow[t]{2}{*}{ Number of Steam Engines } & $0.334^{* * *}$ & $0.402^{* * *}$ & $0.283^{* * *}$ & $0.314^{* * *}$ & $0.291 * * *$ & $0.411^{* * *}$ & $0.117^{* * *}$ & $0.143^{* * *}$ & $0.603^{* * *}$ & $0.643^{* * *}$ & $0.119^{* * *}$ & $0.161^{* * *}$ \\
\hline & {$[0.0912]$} & {$[0.135]$} & {$[0.0534]$} & {$[0.0809]$} & {$[0.0778]$} & {$[0.113]$} & {$[0.0323]$} & {$[0.0451]$} & {$[0.159]$} & {$[0.233]$} & {$[0.0375]$} & {$[0.0516]$} \\
\hline Share of department's territory covered by mines, 1837 & & $\begin{array}{l}-0.0500 \\
{[0.0383]}\end{array}$ & & $\begin{array}{l}-0.0234 \\
{[0.0258]}\end{array}$ & & $\begin{array}{c}-0.0894^{* *} \\
{[0.0351]}\end{array}$ & & $\begin{array}{l}-0.0203 \\
{[0.0145]}\end{array}$ & & $\begin{array}{c}-0.0333 \\
{[0.0746]}\end{array}$ & & $\begin{array}{c}-0.0308^{* *} \\
{[0.0156]}\end{array}$ \\
\hline \multirow[t]{2}{*}{ Average Rainfall } & $1.259^{* * *}$ & $1.216^{* * *}$ & $0.846^{* * *}$ & $0.826^{* * *}$ & $1.014^{* * *}$ & $0.937^{* * *}$ & $0.584^{* * *}$ & $0.566 * * *$ & $1.062^{* *}$ & $1.028^{* *}$ & $0.325^{* * *}$ & $0.298^{* *}$ \\
\hline & {$[0.281]$} & {$[0.290]$} & {$[0.224]$} & {$[0.226]$} & {$[0.257]$} & {$[0.301]$} & {$[0.122]$} & {$[0.131]$} & {$[0.488]$} & {$[0.489]$} & {$[0.120]$} & {$[0.136]$} \\
\hline \multirow[t]{2}{*}{ Average Temperature } & $-1.033^{* *}$ & $-1.217^{* * *}$ & -0.348 & -0.434 & $-1.698^{* * *}$ & $-2.026^{* * *}$ & $-0.995 * * *$ & $-1.070^{* * * *}$ & 1.125 & 0.999 & $-0.478^{* *}$ & $-0.591 * * *$ \\
\hline & {$[0.405]$} & {$[0.423]$} & {$[0.304]$} & {$[0.300]$} & {$[0.425]$} & {$[0.483]$} & {$[0.226]$} & {$[0.247]$} & {$[0.763]$} & {$[0.837]$} & {$[0.192]$} & {$[0.222]$} \\
\hline Latitude & {$[2.522]$} & {$[2.697]$} & {$[1.737]$} & {$[1.823]$} & {$[2.401]$} & {$[2.910]$} & {$[1.190]$} & {$[1.293]$} & [4.320] & {$[4.300]$} & {$[1.234]$} & {$[1.427]$} \\
\hline \multirow[t]{2}{*}{ Land Suitability } & $0.330^{* *}$ & $0.378^{* *}$ & 0.183 & 0.206 & $0.412^{* *}$ & $0.498 * * *$ & $0.209^{* *}$ & $0.229^{* *}$ & 0.0627 & 0.0966 & $0.206^{* * *}$ & $0.235^{* * *}$ \\
\hline & {$[0.150]$} & {$[0.155]$} & {$[0.127]$} & {$[0.126]$} & {$[0.161]$} & {$[0.184]$} & {$[0.0863]$} & {$[0.0926]$} & {$[0.326]$} & {$[0.326]$} & {$[0.0744]$} & {$[0.0808]$} \\
\hline \multirow[t]{2}{*}{ Share of Carboniferous Area } & $-0.675^{* *}$ & -0.457 & -0.150 & -0.0478 & $-0.907^{* * *}$ & $-0.517^{*}$ & $-0.471^{* * *}$ & $-0.382^{* *}$ & -0.449 & -0.302 & $-0.497^{* *}$ & $-0.363^{*}$ \\
\hline & {$[0.283]$} & {$[0.332]$} & {$[0.225]$} & {$[0.241]$} & {$[0.290]$} & {$[0.310]$} & {$[0.152]$} & {$[0.167]$} & {$[0.550]$} & {$[0.662]$} & {$[0.197]$} & {$[0.208]$} \\
\hline \multirow[t]{2}{*}{ Maritime Department } & -0.0140 & -0.0259 & 0.00714 & 0.00180 & $-0.210^{*}$ & -0.232 & -0.0484 & -0.0527 & 0.0304 & 0.0261 & $-0.137^{* *}$ & $-0.145^{* *}$ \\
\hline & {$[0.164]$} & {$[0.178]$} & {$[0.101]$} & {$[0.106]$} & {$[0.128]$} & {$[0.160]$} & {$[0.0572]$} & {$[0.0648]$} & {$[0.262]$} & {$[0.266]$} & {$[0.0609]$} & {$[0.0722]$} \\
\hline \multirow[t]{2}{*}{ Border Department } & -0.0917 & -0.159 & $-0.173^{*}$ & $-0.204^{* *}$ & 0.0218 & -0.0993 & 0.0247 & -0.00270 & $0.881^{1 * * *}$ & $0.837 * * *$ & 0.0269 & -0.0149 \\
\hline & {$[0.121]$} & {$[0.139]$} & {$[0.0907]$} & {$[0.0986]$} & {$[0.119]$} & {$[0.147]$} & {$[0.0564]$} & {$[0.0663]$} & {$[0.277]$} & {$[0.286]$} & {$[0.0624]$} & {$[0.0723]$} \\
\hline \multirow{2}{*}{ Distance to Paris } & 0.00101 & 0.00148 & $0.000982^{*}$ & $0.00120^{*}$ & 0.000474 & 0.00132 & -0.000229 & $-3.83 \mathrm{e}-05$ & -0.00128 & -0.000965 & 0.000122 & 0.000413 \\
\hline & {$[0.000838]$} & {$[0.000964]$} & {$[0.000558]$} & {$[0.000644]$} & {$[0.000716]$} & {$[0.000849]$} & {$[0.000353]$} & {$[0.000373]$} & {$[0.00128]$} & {$[0.00144]$} & {$[0.000393]$} & {$[0.000458]$} \\
\hline Paris and Suburbs & $0.529^{* * *}$ & $0.548^{* * *}$ & $0.690^{* *}$ & $0.699^{* *}$ & 0.100 & 0.134 & -0.0581 & -0.0502 & 0.687 & 0.701 & $0.200^{* *}$ & $0.212^{* *}$ \\
\hline Observations & & First stag & e: the instrun & ented variab & e is Number & of Steam Eng & & & & & & \\
\hline \multirow[t]{2}{*}{ Distance to Fresnes } & $-0.00687^{* * *}$ & $-0.00504^{* *}$ & $-0.00687^{* * *}$ & $-0.00504^{* *}$ & $-0.00687^{* * *}$ & $-0.00504^{* *}$ & $-0.00687^{* * *}$ & $-0.00504^{* *}$ & $-0.00687^{* * *}$ & $-0.00504^{* *}$ & $-0.00687^{* * *}$ & $-0.00504^{* *}$ \\
\hline & {$[0.00250]$} & {$[0.00219]$} & {$[0.00250]$} & {$[0.00219]$} & {$[0.00250]$} & {$[0.00219]$} & {$[0.00250]$} & {$[0.00219]$} & {$[0.00250]$} & {$[0.00219]$} & {$[0.00250]$} & {$[0.00219]$} \\
\hline $\begin{array}{l}\text { Deviation from Wheat Prices in 1835-1838 } \\
\text { (baseline 1810-1829) }\end{array}$ & $\begin{array}{c}-1.633^{* * *} \\
{[0.481]}\end{array}$ & $\begin{array}{c}-1.271^{* * *} \\
{[0.478]}\end{array}$ & $\begin{array}{c}-1.633^{* * *} \\
{[0.481]}\end{array}$ & $\begin{array}{c}-1.271 * * * \\
{[0.478]}\end{array}$ & $\begin{array}{c}-1.633^{* * *} \\
{[0.481]}\end{array}$ & $\begin{array}{c}-1.271 * * * \\
{[0.478]}\end{array}$ & $\begin{array}{c}-1.633^{* * *} \\
{[0.481]}\end{array}$ & $\begin{array}{c}-1.271 * * * \\
{[0.478]}\end{array}$ & $\begin{array}{c}-1.633^{* * *} \\
{[0.481]}\end{array}$ & $\begin{array}{c}-1.271^{* * *} \\
{[0.478]}\end{array}$ & $\begin{array}{c}-1.633^{* * *} \\
{[0.481]}\end{array}$ & $\begin{array}{c}-1.271^{* * *} \\
{[0.478]}\end{array}$ \\
\hline F-stat ( $1^{\text {st }}$ stage $)$ & 13.126 & 9.433 & 13.126 & 9.433 & 13.126 & 9.433 & 13.126 & 9.433 & 13.126 & 9.433 & 13.126 & 9.433 \\
\hline Prob J-Stat & 0.418 & 0.410 & 0.361 & 0.366 & 0.310 & 0.321 & 0.151 & 0.162 & 0.168 & 0.172 & 0.563 & 0.543 \\
\hline
\end{tabular}

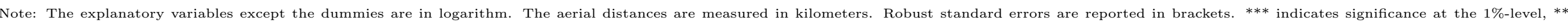
indicates significance at the $5 \%$-level, * indicates significance at the $10 \%$-level. 
Table B.8: Industrialization and human capital formation, accounting for market integration during the French Revolution

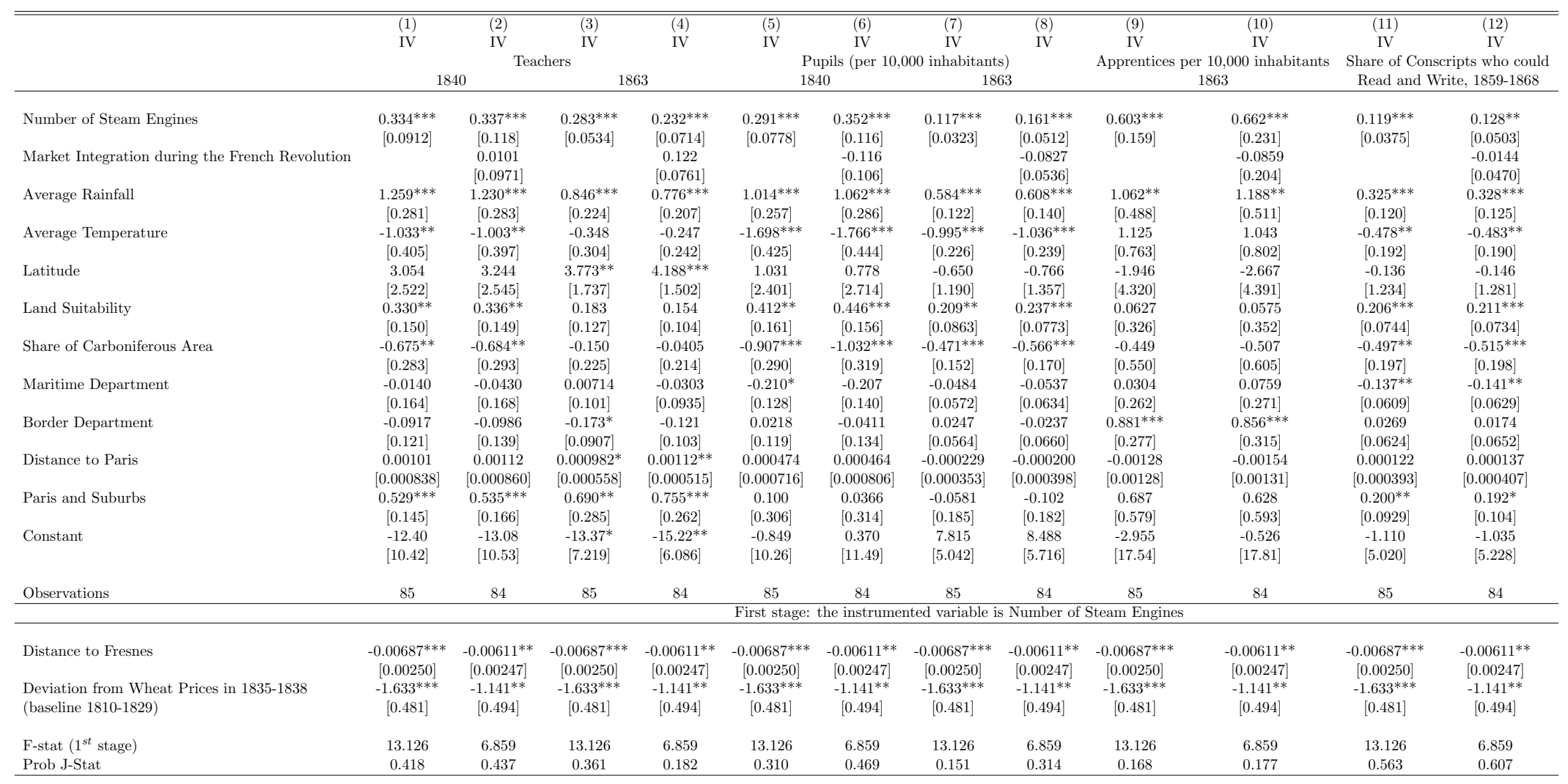

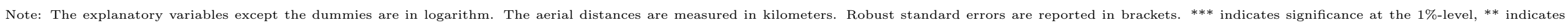
significance at the $5 \%$-level, ${ }^{*}$ indicates significance at the $10 \%$-level. 
Table B.9: Industrialization and human capital formation, accounting for railroad connection to Paris in 1860

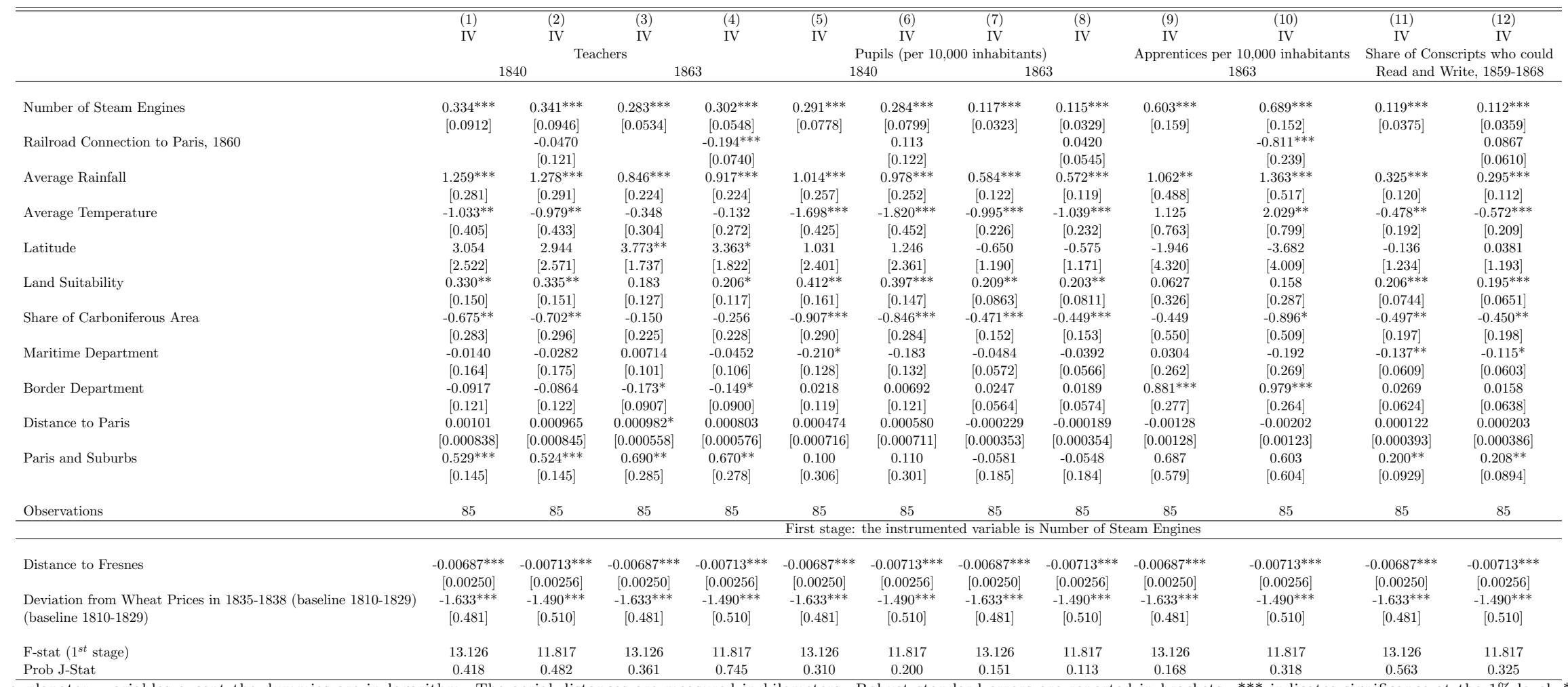

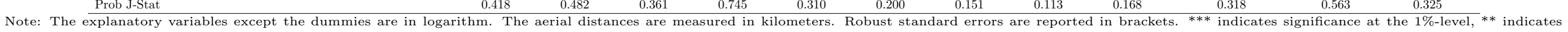
significance at the $5 \%$-level, $*$ indicates significance at the $10 \%$-level. 
Table B.10: Industrialization and human capital formation, accounting for population density in the $19^{\text {th }}$ century

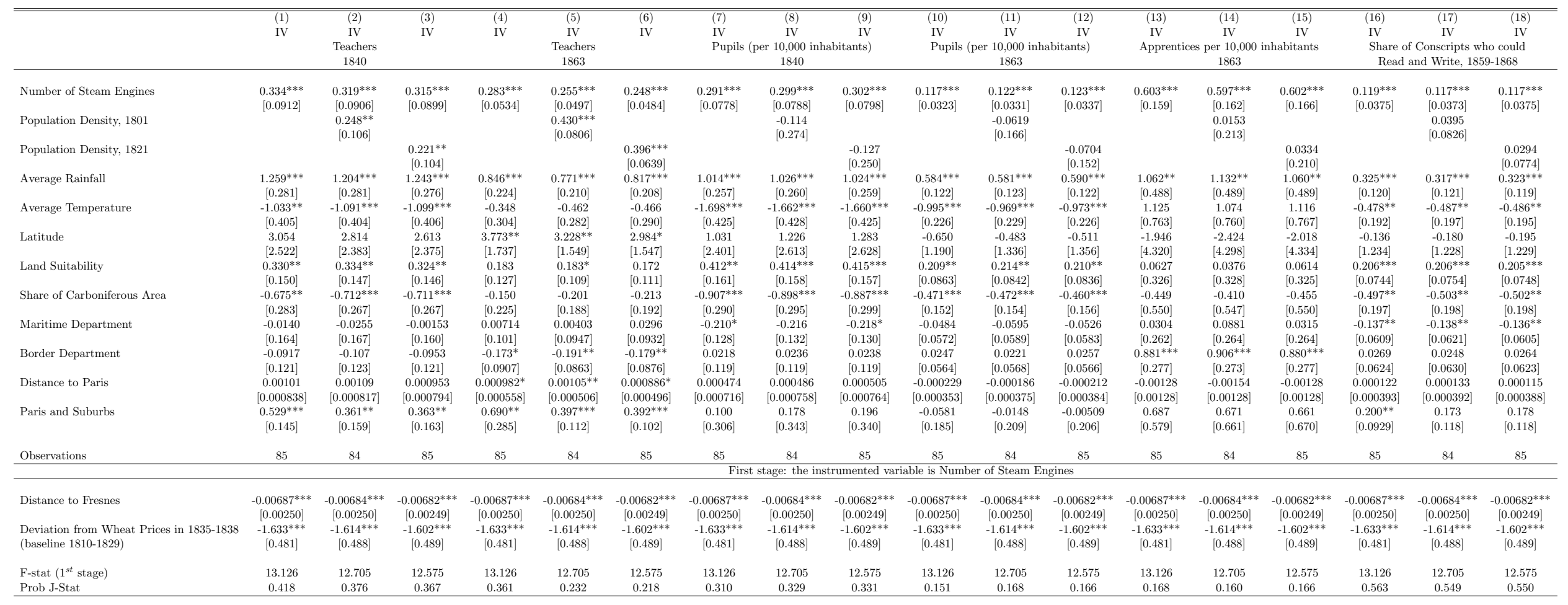

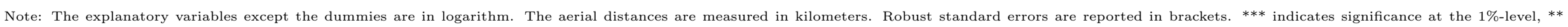

indicates significance at the $5 \%$-level, $*$ indicates significance at the $10 \%$-level. 
Table B.11: Industrialization and human capital formation, accounting for fertility in 1806

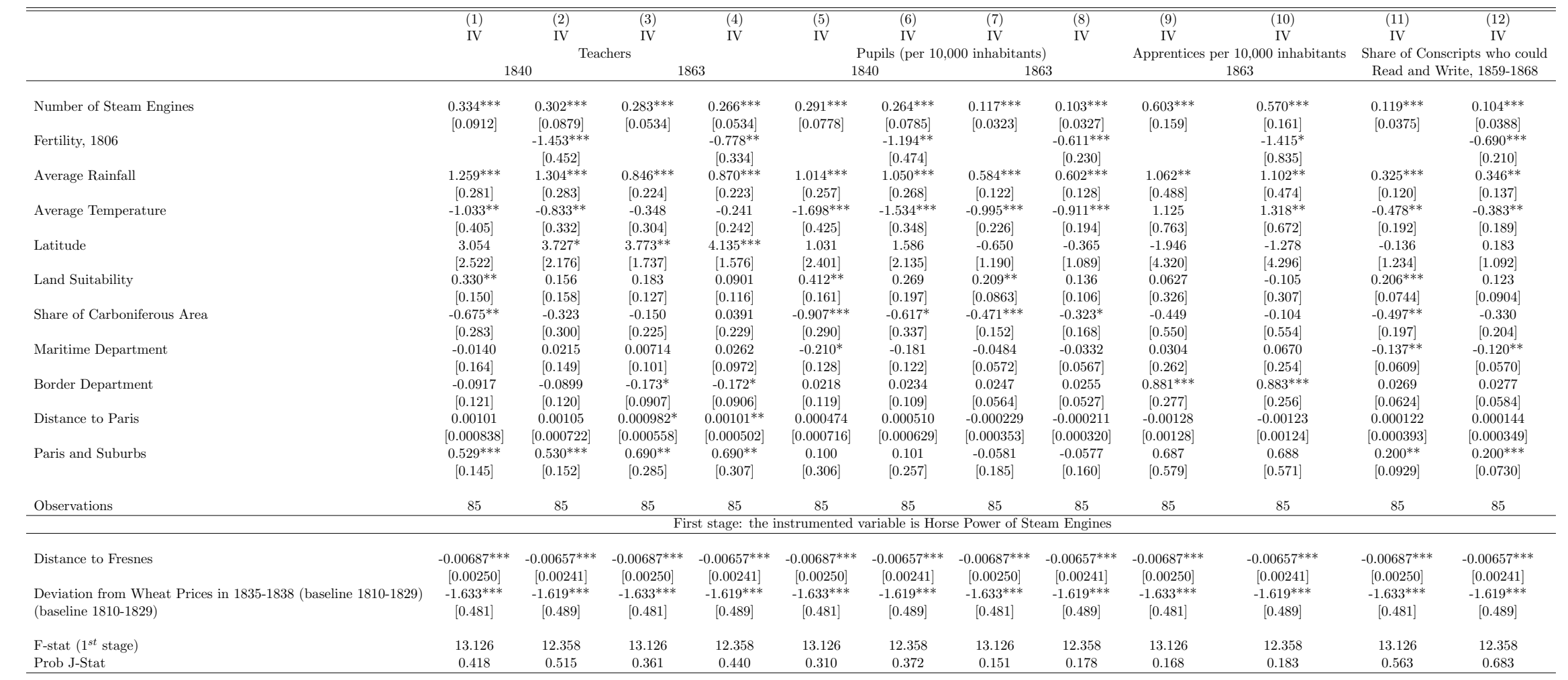

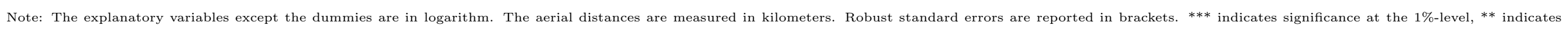
significance at the $5 \%$-level, ${ }^{*}$ indicates significance at the $10 \%$-level. 
Table B.12: The effect of industrialization on wages, 1839-1847

\begin{tabular}{lccc}
\hline \hline & $(1)$ & $(2)$ & $(3)$ \\
& OLS & OLS & OLS \\
& Average Male Wage, $1839-1847$ & Average Female Wage, $1839-1847$ & Average Child Wage, $1839-1847$ \\
\hline \multirow{2}{*}{ Number of Steam Engines } & & & \\
& $0.0615^{* * *}$ & $0.0645^{* * *}$ & $0.0526^{* *}$ \\
Average Rainfall & {$[0.0132]$} & {$[0.0157]$} & {$[0.0214]$} \\
& -0.0385 & 0.292 & 0.0844 \\
Average Temperature & {$[0.136]$} & {$[0.179]$} & {$[0.197]$} \\
& -0.0698 & 0.134 & 0.0531 \\
Latitude & {$[0.125]$} & {$[0.157]$} & {$[0.184]$} \\
& $-2.136^{* *}$ & 0.178 & -1.441 \\
Land Suitability & {$[0.820]$} & {$[1.217]$} & {$[1.317]$} \\
Share of Carboniferous & 0.0776 & 0.0282 & 0.0772 \\
& {$[0.0688]$} & {$[0.0932]$} & {$[0.0924]$} \\
Maritime Department & -0.0105 & -0.192 & -0.0479 \\
& {$[0.158]$} & {$[0.240]$} & {$[0.238]$} \\
Border Department & 0.00259 & -0.0427 & -0.00610 \\
& {$[0.0446]$} & {$[0.0580]$} & {$[0.0586]$} \\
Distance to Paris & 0.0515 & -0.0246 & -0.0468 \\
Paris and Suburbs & {$[0.0497]$} & {$[0.0584]$} & {$[0.0699]$} \\
& -0.000263 & $-6.10 \mathrm{e}-05$ & -0.000291 \\
& {$[0.000272]$} & {$[0.000390]$} & {$[0.000417]$} \\
Adjusted R2 & $0.343^{* * *}$ & $0.363^{* * *}$ & $0.297^{* * *}$ \\
Observations & {$[0.0986]$} & {$[0.0739]$} & {$[0.0891]$} \\
& & & \\
& 0.349 & 0.235 & 0.163 \\
& 85 & 85 & 85
\end{tabular}

Note: The explanatory variables except the dummies are in logarithm. The aerial distances are measured in kilometers. Robust standard errors are reported in brackets. $* * *$ indicates significance at the $1 \%$-level, $* *$ indicates significance at the $5 \%$-level, $*$ indicates significance at the $10 \%$-level. 
Table B.13: Industrialization and human capital formation, accounting for wages in 1839-1847

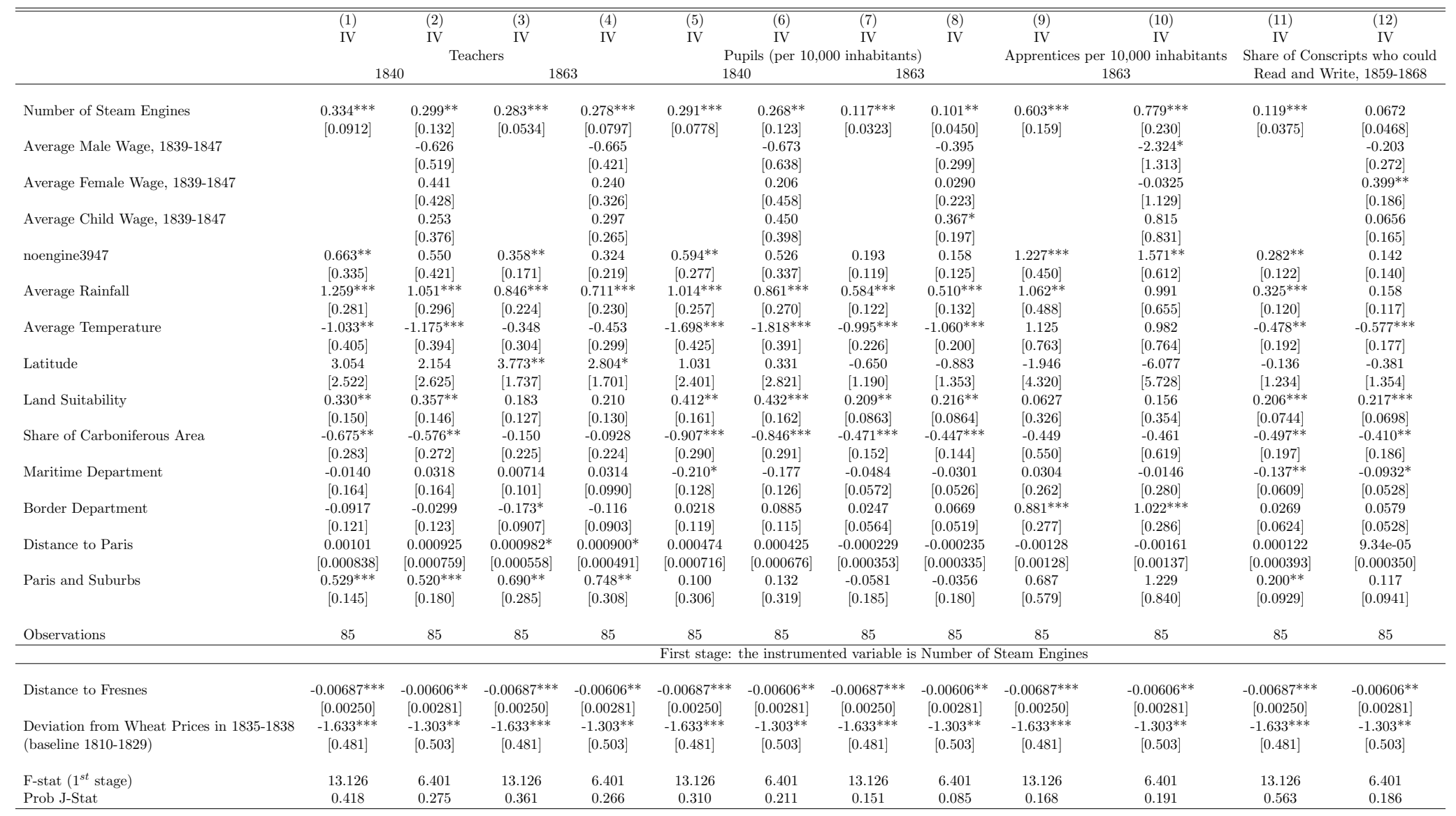

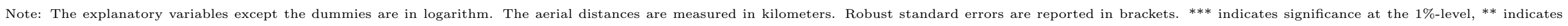
significance at the $5 \%$-level, * indicates significance at the $10 \%$-level. 
Table B.14: Industrialization and human capital formation, accounting for the upper tail of the human capital distribution in the $18^{\text {th }}$ century

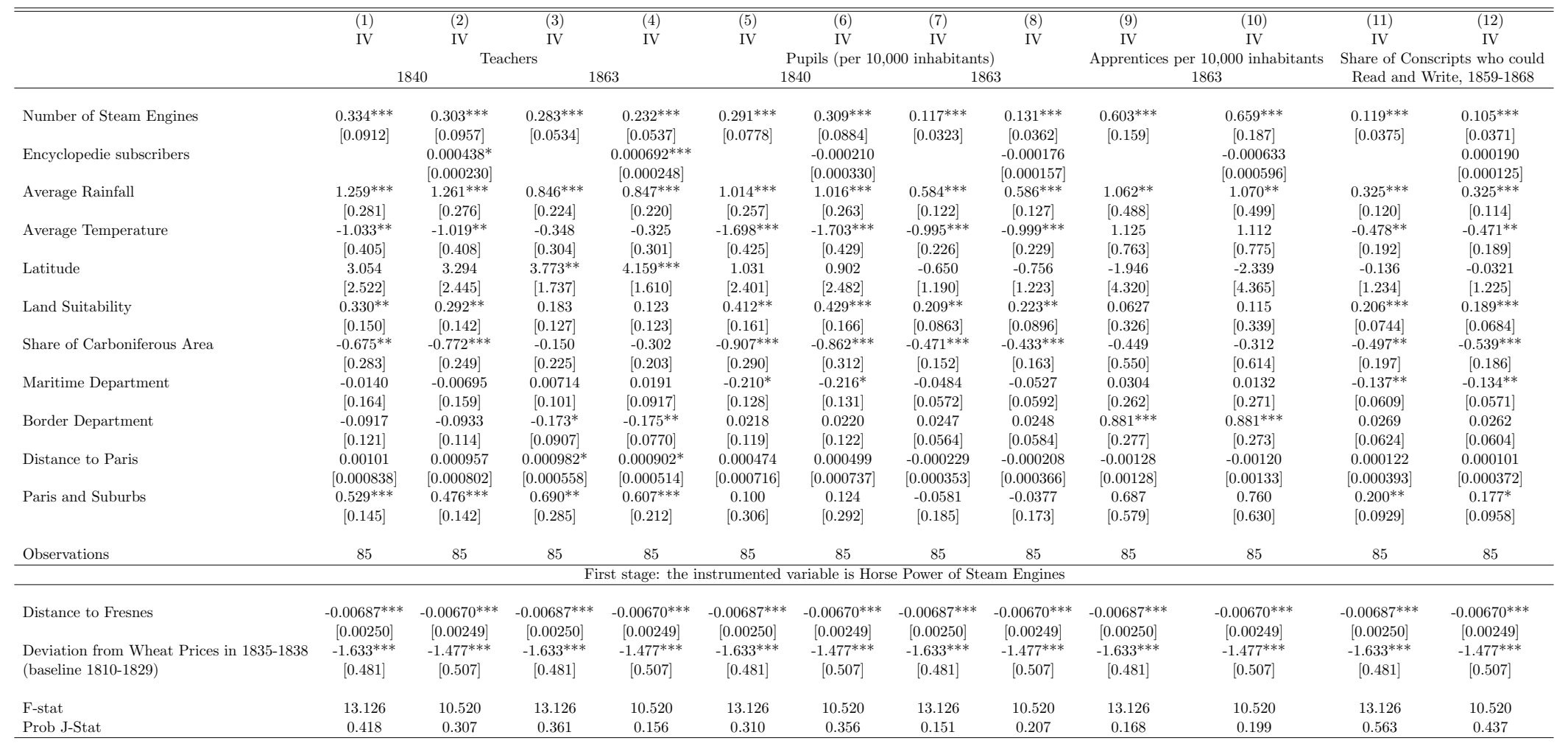

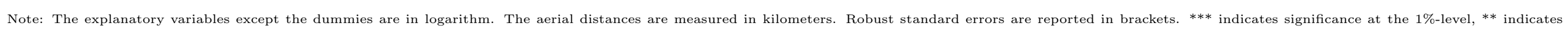
significance at the $5 \%$-level, * indicates significance at the $10 \%$-level. 


\section{Appendix C. Literacy of Conscripts, 1847-1856}

Table C.1: The effect of industrialization on the share of literate conscripts, 1847-1856

\begin{tabular}{|c|c|c|c|c|c|c|c|c|c|c|c|c|}
\hline & $\begin{array}{l}(1) \\
\text { OLS }\end{array}$ & $\begin{array}{c}(2) \\
\text { OLS }\end{array}$ & $\begin{array}{c}(3) \\
\text { OLS }\end{array}$ & $\begin{array}{l}(4) \\
\text { OLS }\end{array}$ & $\begin{array}{c}(5) \\
\text { OLS }\end{array}$ & $\begin{array}{ll}(6) \\
\text { OLS }\end{array}$ & $\begin{array}{ll}(7) \\
\text { OLS }\end{array}$ & $\begin{array}{ll}(8) \\
\text { IV }\end{array}$ & $\begin{array}{l}(9) \\
\text { IV }\end{array}$ & $\begin{array}{c}(10) \\
\text { IV }\end{array}$ & $\begin{array}{l}\text { (11) } \\
\text { IV }\end{array}$ & $\begin{array}{l}(12) \\
\text { IV }\end{array}$ \\
\hline & & & & & \multicolumn{8}{|c|}{$\begin{array}{l}\text { OLS OLS OLS IV } \\
\text { Share of Conscripts who could Read and Write, } 1847-1856\end{array}$} \\
\hline Number of Steam Engines & $\begin{array}{c}0.0723^{* *} \\
{[0.0280]}\end{array}$ & $\begin{array}{c}0.0573 * * \\
{[0.0277]}\end{array}$ & $\begin{array}{c}0.0550^{* *} \\
{[0.0259]}\end{array}$ & $\begin{array}{l}0.0405^{* *} \\
{[0.0189]}\end{array}$ & $\begin{array}{l}0.0630^{* *} \\
{[0.0254]}\end{array}$ & $\begin{array}{l}0.0523^{*} \\
0.0285]\end{array}$ & $\begin{array}{l}0.0438 * * \\
{[0.0209]}\end{array}$ & $\begin{array}{l}0.149^{* * *} \\
{[0.0496]}\end{array}$ & $\begin{array}{l}0.0918^{* *} \\
{[0.0380]}\end{array}$ & $\begin{array}{l}0.152^{* * *} \\
0.04681\end{array}$ & $\begin{array}{l}0.165^{* * *} \\
0.0554]\end{array}$ & $\begin{array}{l}0.105^{* * *} \\
0.04011\end{array}$ \\
\hline Average Rainfall & & $\begin{array}{l}0.207 \\
{[0.170]}\end{array}$ & $\begin{array}{c}0.374^{* *} \\
{[0.158]}\end{array}$ & $\begin{array}{l}0.0793 \\
{[0.169]}\end{array}$ & $\begin{array}{l}0.358^{* *} \\
{[0.158]}\end{array}$ & $\begin{array}{l}0.393^{* *} \\
{[0.168]}\end{array}$ & $\begin{array}{l}0.0658 \\
{[0.162]}\end{array}$ & $\begin{array}{c}0.473 * * * \\
{[0.153]}\end{array}$ & $\begin{array}{l}0.158 \\
{[0.167]}\end{array}$ & $\begin{array}{l}0.429 * * * \\
{[0.151]}\end{array}$ & $\begin{array}{l}0.391 * * \\
{[0.164]}\end{array}$ & 0.0896 \\
\hline Average Temperature & & $-1.071^{* * *}$ & $-0.776^{* * *}$ & -0.134 & $-0.738^{* * *}$ & $-0.770^{* * *}$ & -0.138 & $-0.702^{* * *}$ & -0.0938 & $-0.640 * * *$ & $-0.726^{* * * *}$ & -0.115 \\
\hline & & {$[0.261]$} & {$[0.207]$} & {$[0.264]$} & {$[0.205]$} & {$[0.206]$} & {$[0.270]$} & {$[0.215]$} & {$[0.246]$} & {$[0.204]$} & {$[0.212]$} & {$[0.241]$} \\
\hline Latitude & & 0.0946 & 0.870 & -0.531 & 0.799 & 0.880 & -0.608 & 0.429 & -0.625 & 0.349 & 0.365 & -0.928 \\
\hline & & {$[0.703]$} & {$[1.752]$} & {$[1.575]$} & {$[1.814]$} & {$[1.749]$} & [1.571] & {$[1.604]$} & {$[1.400]$} & {$[1.727]$} & {$[1.700]$} & {$[1.424]$} \\
\hline Land Suitability & & $\begin{array}{c}0.482^{* * *} \\
{[0.131]}\end{array}$ & $\begin{array}{l}0.313^{* * *} \\
{[0.0980]}\end{array}$ & $\begin{array}{l}0.141 \\
{[0.108]}\end{array}$ & $\begin{array}{l}0.315^{* * *} \\
{[0.0972]}\end{array}$ & $\begin{array}{c}0.312^{* * *} \\
{[0.0975]}\end{array}$ & $\begin{array}{l}0.144 \\
{[0.111]}\end{array}$ & $\begin{array}{c}0.282 * * * \\
{[0.0928]}\end{array}$ & $\begin{array}{c}0.128 \\
{[0.0994]}\end{array}$ & $\begin{array}{c}0.289^{* * *} \\
{[0.0904]}\end{array}$ & $\begin{array}{l}0.285^{* * *} \\
{[0.0929]}\end{array}$ & $\begin{array}{c}0.136 \\
{[0.0975]}\end{array}$ \\
\hline Share of Carboniferous Area & & & $-0.604^{* *}$ & $\begin{array}{c}-0.473^{* *} \\
\end{array}$ & $-0.633^{* * *}$ & $-0.603^{* *}$ & $-0.478^{* *}$ & $-0.630^{* * * *}$ & $\begin{array}{l}-0.490^{* *} \\
\end{array}$ & $-0.682^{* * *}$ & $-0.638^{* * * *}$ & $-0.506^{* *}$ \\
\hline Maritime Department & & & -0.0501 & $-0.120^{*}$ & -0.0541 & -0.0528 & -0.116 & -0.120 & $-0.163^{* *}$ & -0.118 & -0.110 & $-0.144^{*}$ \\
\hline & & & {$[0.0675]$} & {$[0.0701]$} & {$[0.0687]$} & {$[0.0676]$} & {$[0.0770]$} & {$[0.0804]$} & {$[0.0767]$} & {$[0.0795]$} & {$[0.0817]$} & {$[0.0787]$} \\
\hline Border Department & & & 0.0894 & -0.0511 & 0.0977 & 0.0912 & -0.0496 & 0.0690 & -0.0531 & 0.0876 & 0.0602 & $\begin{array}{l}-0.0505 \\
-0.05551\end{array}$ \\
\hline Distance to Paris & & & $\begin{array}{l}{[0.0637]} \\
0.000167\end{array}$ & $\begin{array}{l}{[0.0526]} \\
0.000125\end{array}$ & $\begin{array}{l}{[0.0634]} \\
0.000175\end{array}$ & $\begin{array}{l}{[0.0635]} \\
0.000161\end{array}$ & $\begin{array}{l}{[0.0554]} \\
0.000114\end{array}$ & $\begin{array}{l}{[0.0743]} \\
0.000218\end{array}$ & $\begin{array}{l}{[0.0522]} \\
0.000168\end{array}$ & $\begin{array}{c}{[0.0699]} \\
0.000227\end{array}$ & $\begin{array}{l}{[0.0761]} \\
0.000244\end{array}$ & $\begin{array}{r}{[0.0555]} \\
0.000129\end{array}$ \\
\hline Distance to Paris & & & {$[0.000512]$} & {$\left[\begin{array}{l}0.0000420 \\
{[0.00437]}\end{array}\right.$} & {$[0.000522]$} & $\begin{array}{l}0.000161 \\
{[0.000510]}\end{array}$ & {$\left[\begin{array}{l}0.0000144 \\
{[0.00043]}\end{array}\right]$} & $\begin{array}{l}0.000018 \\
{[0.000498]}\end{array}$ & $\begin{array}{l}0.000168 \\
{[0.000399]}\end{array}$ & {$[0.000511]$} & {$\left[\begin{array}{l}0.000244 \\
{[0.000518]}\end{array}\right.$} & {$\left[\begin{array}{l}0.0000129 \\
{[0.000411]}\end{array}\right]$} \\
\hline Paris and Suburbs & & & $\begin{array}{c}0.341^{* * *} \\
{[0.0994]}\end{array}$ & $\begin{array}{c}0.125 \\
{[0.0823]}\end{array}$ & $\begin{array}{c}0.346 * * * \\
{[0.107]}\end{array}$ & $\begin{array}{c}0.339 * * * \\
{[0.0990]}\end{array}$ & $\begin{array}{c}0.119 \\
{[0.0809]}\end{array}$ & $\begin{array}{c}0.309^{* * *} \\
{[0.117]}\end{array}$ & $\begin{array}{c}0.138 \\
{[0.0982]}\end{array}$ & $\begin{array}{c}0.322^{* * *} \\
{[0.119]}\end{array}$ & $\begin{array}{c}0.318^{* * *} \\
{[0.114]}\end{array}$ & $\begin{array}{c}0.111 \\
{[0.0926]}\end{array}$ \\
\hline Grooms who Signed their Marriage License, $1786-1790$ & & & & $\begin{array}{c}0.810^{* * *} \\
{[0.195]}\end{array}$ & & & $\begin{array}{c}0.804^{* * *} \\
{[0.201]}\end{array}$ & & $\begin{array}{c}0.785^{* * *} \\
{[0.188]}\end{array}$ & & & $\begin{array}{c}0.770^{* * *} \\
{[0.182]}\end{array}$ \\
\hline University & & & & & $\begin{array}{l}-0.0720 \\
{[0.0657]}\end{array}$ & & $\begin{array}{l}-0.0163 \\
{[0.0584]}\end{array}$ & & & $\begin{array}{l}-0.139^{*} \\
{[0.0716]}\end{array}$ & & $\begin{array}{l}-0.0539 \\
{[0.0593]}\end{array}$ \\
\hline Urban Population in 1700 & & & & & & $\begin{array}{l}0.00489 \\
{[0.0178]}\end{array}$ & $\begin{array}{l}-0.00292 \\
{[0.0176]}\end{array}$ & & & & $\begin{array}{l}-0.0221 \\
{[0.0185]}\end{array}$ & $\begin{array}{l}-0.0152 \\
{[0.0150]}\end{array}$ \\
\hline Adjusted R2 & 0.070 & 0.331 & 0.436 & 0.625 & 0.436 & 0.429 & 0.614 & & & & & \\
\hline Observations & 85 & 85 & 85 & 79 & 85 & 85 & 79 & 85 & 79 & 85 & 85 & 79 \\
\hline & & & & First s & stage: the ins & trumented v & riable is $\mathrm{Ho}$ & ise Power of & team Engines & & & \\
\hline Distance to Fresnes & & & & & & & & $\begin{array}{c}-0.00687 * * * \\
{[0.00250]}\end{array}$ & $\begin{array}{c}-0.00682^{* *} \\
{[0.00266]}\end{array}$ & $\begin{array}{c}-0.00702^{* * *} \\
{[0.00211]}\end{array}$ & $\begin{array}{c}-0.00585^{* *} \\
{[0.00245]}\end{array}$ & $\begin{array}{c}-0.00610^{* * *} \\
{[0.00230]}\end{array}$ \\
\hline $\begin{array}{l}\text { Deviation from Wheat Prices in 1835-1838 } \\
\text { (baseline } 1810-1829 \text { ) }\end{array}$ & & & & & & & & $\begin{array}{l}-1.633^{* * *} \\
{[0.481]}\end{array}$ & $\begin{array}{c}-1.608^{* * *} \\
{[0.527]}\end{array}$ & $\begin{array}{l}-1.571^{* * *} * \\
{[0.493]}\end{array}$ & $\begin{array}{c}-1.463^{* * *} \\
{[0.511]}\end{array}$ & $\begin{array}{c}-1.401 * * \\
{[0.557]}\end{array}$ \\
\hline F-stat ( $1^{s t}$ stage $)$ & & & & & & & & 13.126 & 10.072 & 14.403 & 8.658 & 8.043 \\
\hline Prob J-Stat & & & & & & & & 0.337 & 0.203 & 0.360 & 0.323 & 0.211 \\
\hline
\end{tabular}

Note: The explanatory variables except the dummies are in logarithm. The aerial distances are measured in kilometers. Robust standard errors are reported in brackets. ${ }^{* * *}$ indicates significance

at the $1 \%$-level, $* *$ indicates significance at the $5 \%$-level, $*$ indicates significance at the $10 \%$-level. 


\section{Appendix D. Schools Buildings and Public Spending on Education}

Table D.1: The effect of industrialization on public spending on education, 1855-1863

\begin{tabular}{|c|c|c|c|c|c|c|c|c|c|c|c|c|c|}
\hline & $\begin{array}{l}(1) \\
\text { OLS }\end{array}$ & $\begin{array}{l}(2) \\
\text { OLS }\end{array}$ & $\begin{array}{c}(3) \\
\text { OLS }\end{array}$ & $\begin{array}{l}(4) \\
\text { OLS }\end{array}$ & $\begin{array}{c}\text { OLS } \\
\text { OLS } \\
\text { al Public Spes }\end{array}$ & $\begin{array}{c}\text { (6) } \\
\text { OLS } \\
\text { dding on Prime }\end{array}$ & $\begin{array}{c}\text { (7) } \\
\text { OLS } \\
\text { ary Schooling } \mathrm{p}\end{array}$ & $\begin{array}{c}(8) \\
\text { IV } \\
\text { er Inhabitant, } 1\end{array}$ & $\begin{array}{r}(9) \\
\text { IV } \\
855-1863\end{array}$ & $\begin{array}{l}(10) \\
\text { IV }\end{array}$ & $\begin{array}{l}\text { (11) } \\
\text { IV }\end{array}$ & $\begin{array}{l}(12) \\
\text { IV }\end{array}$ & \\
\hline Number of Steam Engines & $\begin{array}{l}-0.0130 \\
{[0.0132]}\end{array}$ & $\begin{array}{c}-0.0280^{* *} \\
{[0.0137]}\end{array}$ & $\begin{array}{c}-0.0248^{*} \\
{[0.0138]}\end{array}$ & $\begin{array}{c}-0.0232 * * \\
{[0.00961}\end{array}$ & $\begin{array}{l}-0.0148 \\
{[0.01271}\end{array}$ & $\begin{array}{r}-0.0123 \\
{[0.01191}\end{array}$ & $\begin{array}{c}-0.0129 \\
{[0.00945}\end{array}$ & $\begin{array}{l}-0.0148^{*} \\
{[0.00868]}\end{array}$ & $\begin{array}{l}0.0236 \\
0\end{array}$ & 0.00337 & 0.0267 & $0.0506^{*}$ & 0.0205 \\
\hline Average Rainfall & & $\begin{array}{l}0.0776 \\
{[0.0875]}\end{array}$ & $\begin{array}{l}0.190^{* *} \\
{[0.0809]}\end{array}$ & $\begin{array}{c}0.0485 \\
{[0.0842]}\end{array}$ & $\begin{array}{l}0.169^{* *} \\
{[0.0823]}\end{array}$ & 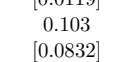 & $\begin{array}{l}-0.0154 \\
{[0.0829]}\end{array}$ & $\begin{array}{l}-0.0173 \\
{[0.0814]}\end{array}$ & $\begin{array}{c}0.241^{* * *} \\
{[0.0827]}\end{array}$ & $\begin{array}{c}0.0893 \\
{[0.0855]}\end{array}$ & $\begin{array}{l}0.202^{* *} \\
{[0.0833]}\end{array}$ & $\begin{array}{c}0.0202 \\
0.102 \\
{[0.0853]}\end{array}$ & $\begin{array}{c}-0.00244 \\
{[0.0831]}\end{array}$ \\
\hline Average Temperature & & $\begin{array}{c}-0.700^{* * *} \\
{[0.175]}\end{array}$ & $\begin{array}{c}-0.513^{* * *} \\
{[0.157]}\end{array}$ & $\begin{array}{l}-0.210 \\
{[0.161]}\end{array}$ & $\begin{array}{c}-0.466^{* * *} \\
{[0.157]}\end{array}$ & $\begin{array}{c}-0.540^{* * *} \\
{[0.148]}\end{array}$ & $\begin{array}{l}-0.227 \\
{[0.149]}\end{array}$ & $\begin{array}{l}-0.227 \\
{[0.149]}\end{array}$ & $\begin{array}{c}-0.475^{* * *} \\
{[0.169]}\end{array}$ & $\begin{array}{l}-0.189 \\
{[0.160]}\end{array}$ & $\begin{array}{c}-0.420^{* *} \\
{[0.165]}\end{array}$ & $\begin{array}{c}-0.516^{* * * *} \\
{[0.158]}\end{array}$ & $\begin{array}{l}-0.215 \\
{[0.141]}\end{array}$ \\
\hline Latitude & & $\begin{array}{l}-0.360 \\
{[0.367]}\end{array}$ & $\begin{array}{c}-1.682^{* * *} \\
{[0.599]}\end{array}$ & $\begin{array}{c}-2.299 * * * \\
{[0.598]}\end{array}$ & $\begin{array}{c}-1.771^{* * *} \\
{[0.666]}\end{array}$ & $\begin{array}{c}-1.729^{* *} \\
{[0.666]}\end{array}$ & $\begin{array}{c}-2.501^{* * *} \\
{[0.619]}\end{array}$ & $\begin{array}{c}-2.437^{* * *} \\
{[0.609]}\end{array}$ & $\begin{array}{c}-1.908^{* * *} \\
{[0.575]}\end{array}$ & $\begin{array}{c}-2.348^{* * * *} \\
{[0.560]}\end{array}$ & $\begin{array}{c}-1.981^{* * * *} \\
{[0.681]}\end{array}$ & $\begin{array}{c}-2.017^{* * * *} \\
{[0.708]}\end{array}$ & $\begin{array}{c}-2.675^{* * *} \\
{[0.572]}\end{array}$ \\
\hline Land Suitability & & $\begin{array}{c}0.248^{* *} \\
{[0.101]}\end{array}$ & $\begin{array}{c}0.146^{*} \\
{[0.0817]}\end{array}$ & $\begin{array}{c}0.0439 \\
{[0.0760]}\end{array}$ & $\begin{array}{c}0.148^{*} \\
{[0.0775]}\end{array}$ & $\begin{array}{l}0.151^{* *} \\
{[0.0752]}\end{array}$ & $\begin{array}{c}0.0481 \\
{[0.0681]}\end{array}$ & $\begin{array}{c}0.0455 \\
{[0.0684]}\end{array}$ & $\begin{array}{c}0.130^{*} \\
{[0.0765]}\end{array}$ & $\begin{array}{c}0.0369 \\
{[0.0701]}\end{array}$ & $\begin{array}{c}0.136^{*} \\
{[0.0705]}\end{array}$ & $\begin{array}{l}0.136^{* *} \\
{[0.0660]}\end{array}$ & $\begin{array}{c}0.0439 \\
{[0.0575]}\end{array}$ \\
\hline Share of Carboniferous Area & & & $\begin{array}{c}-0.296^{* * *} \\
{[0.105]}\end{array}$ & $\begin{array}{c}-0.212^{* *} \\
{[0.0859]}\end{array}$ & $\begin{array}{c}-0.332^{* * *} \\
{[0.105]}\end{array}$ & $\begin{array}{c}-0.302^{* * *} \\
{[0.105]}\end{array}$ & $\begin{array}{c}-0.216^{* *} \\
{[0.0855]}\end{array}$ & $\begin{array}{c}-0.207^{* *} \\
{[0.0831]}\end{array}$ & $\begin{array}{c}-0.309^{* * * *} \\
{[0.0966]}\end{array}$ & $\begin{array}{c}-0.220^{* * * *} \\
{[0.0805]}\end{array}$ & $\begin{array}{c}-0.355^{* * * *} \\
{[0.0976]}\end{array}$ & $\begin{array}{c}-0.322^{* * * *} \\
{[0.105]}\end{array}$ & $\begin{array}{c}-0.231^{* * *} \\
{[0.0824]}\end{array}$ \\
\hline Maritime Department & & & $\begin{array}{c}-0.00831 \\
{[0.0310]}\end{array}$ & $\begin{array}{l}-0.0352 \\
{[0.0331]}\end{array}$ & $\begin{array}{l}-0.0133 \\
{[0.0339]}\end{array}$ & $\begin{array}{l}0.00372 \\
{[0.0324]}\end{array}$ & $\begin{array}{l}-0.0204 \\
{[0.0322]}\end{array}$ & $\begin{array}{l}-0.0220 \\
{[0.0317]}\end{array}$ & $\begin{array}{l}-0.0441 \\
{[0.0365]}\end{array}$ & $\begin{array}{l}-0.0574 \\
{[0.0352]}\end{array}$ & $\begin{array}{l}-0.0432 \\
{[0.0378]}\end{array}$ & $\begin{array}{l}-0.0285 \\
{[0.0409]}\end{array}$ & $\begin{array}{l}-0.0358 \\
{[0.0338]}\end{array}$ \\
\hline Border Department & & & $\begin{array}{c}0.0588^{* *} \\
{[0.0289]}\end{array}$ & $\begin{array}{c}0.0117 \\
{[0.0294]}\end{array}$ & $\begin{array}{c}0.0692^{* *} \\
{[0.0269]}\end{array}$ & $\begin{array}{l}0.0507^{*} \\
{[0.0273]}\end{array}$ & $\begin{array}{l}0.00886 \\
{[0.0303]}\end{array}$ & $\begin{array}{l}0.00453 \\
{[0.0291]}\end{array}$ & $\begin{array}{c}0.0482 \\
{[0.0322]}\end{array}$ & $\begin{array}{c}0.0107 \\
{[0.0284]}\end{array}$ & $\begin{array}{c}0.0645^{* *} \\
{[0.0275]}\end{array}$ & $\begin{array}{c}0.0334 \\
{[0.0337]}\end{array}$ & $\begin{array}{l}0.00834 \\
{[0.0283]}\end{array}$ \\
\hline Distance to Paris & & & $\begin{array}{c}-0.000484^{* * *} \\
{[0.000172]}\end{array}$ & $\begin{array}{c}-0.000518^{* * *} \\
{[0.000168]}\end{array}$ & $\begin{array}{c}-0.000474^{* *} \\
{[0.000185]}\end{array}$ & $\begin{array}{c}-0.000460^{* *} \\
{[0.000183]}\end{array}$ & $\begin{array}{c}-0.000538^{* * * *} \\
{[0.000175]}\end{array}$ & $\begin{array}{c}-0.000525^{* * *} \\
{[0.000173]}\end{array}$ & $\begin{array}{c}-0.000458^{* *} \\
{[0.000180]}\end{array}$ & $\begin{array}{c}-0.000496^{* * *} \\
{[0.000161]}\end{array}$ & $\begin{array}{c}-0.000450^{* *} \\
{[0.000195]}\end{array}$ & $\begin{array}{c}-0.000414^{* *} \\
{[0.000209]}\end{array}$ & $\begin{array}{c}-0.000530^{* * *} \\
{[0.000166]}\end{array}$ \\
\hline Paris and Suburbs & & & $\begin{array}{l}-0.100 \\
{[0.190]}\end{array}$ & $\begin{array}{c}0.0300 \\
{[0.0352]}\end{array}$ & $\begin{array}{c}-0.0951 \\
{[0.169]}\end{array}$ & $\begin{array}{c}-0.0903 \\
{[0.164]}\end{array}$ & $\begin{array}{l}0.00890 \\
{[0.0332]}\end{array}$ & $\begin{array}{c}0.0118 \\
{[0.0323]}\end{array}$ & $\begin{array}{l}-0.117 \\
{[0.195]}\end{array}$ & $\begin{array}{c}0.0366 \\
{[0.0439]}\end{array}$ & $\begin{array}{l}-0.106 \\
{[0.164]}\end{array}$ & $\begin{array}{l}-0.102 \\
{[0.155]}\end{array}$ & $\begin{array}{l}0.00452 \\
{[0.0368]}\end{array}$ \\
\hline Grooms who Signed their Marriage License, $1786-1790$ & & & & $\begin{array}{l}0.357^{* * *} \\
{[0.0724]}\end{array}$ & & & $\begin{array}{c}0.355^{* * * *} \\
{[0.0713]}\end{array}$ & $\begin{array}{c}0.366^{* * * *} \\
{[0.0680]}\end{array}$ & & $\begin{array}{c}0.344^{* * * *} \\
{[0.0724]}\end{array}$ & & & $\begin{array}{c}0.336^{* * * *} \\
{[0.0688]}\end{array}$ \\
\hline University & & & & & $\begin{array}{c}-0.09066^{* *} \\
{[0.0366]}\end{array}$ & & $\begin{array}{l}-0.0226 \\
{[0.0261]}\end{array}$ & & & & $\begin{array}{c}-0.122^{* * * *} \\
{[0.0390]}\end{array}$ & & $\begin{array}{l}-0.0431 \\
{[0.0265]}\end{array}$ \\
\hline Urban Population in 1700 & & & & & & $\begin{array}{c}-0.0223^{* *} \\
{[0.0104]}\end{array}$ & $\begin{array}{c}-0.0152^{* * *} \\
{[0.00679]}\end{array}$ & $\begin{array}{c}-0.0162^{* *} \\
{[0.00658]}\end{array}$ & & & & $\begin{array}{c}-0.0375^{* * *} \\
{[0.0128]}\end{array}$ & $\begin{array}{c}-0.0219^{* * * *} \\
{[0.00770]}\end{array}$ \\
\hline $\begin{array}{l}\text { Adjusted R2 } \\
\text { Observations }\end{array}$ & $\begin{array}{c}0.001 \\
85\end{array}$ & $\begin{array}{c}0.346 \\
85\end{array}$ & $\begin{array}{l}0.427 \\
85\end{array}$ & $\begin{array}{c}0.670 \\
79\end{array}$ & $\begin{array}{c}0.469 \\
85\end{array}$ & $\begin{array}{c}0.464 \\
85\end{array}$ & $\begin{array}{c}0.687 \\
79\end{array}$ & $\begin{array}{c}0.689 \\
79\end{array}$ & 85 & 79 & 85 & 85 & 79 \\
\hline & & & & & irst stage: the & instrumented & variable is Nun & nber of Steam $\mathrm{F}$ & Engines & & & & \\
\hline $\begin{array}{l}\text { Deviation from Wheat Prices in 1835-1838 } \\
\text { (baseline 1810-1829) }\end{array}$ & & & & & & & & & $\begin{array}{c}-0.00687^{* * *} \\
{[0.00250]} \\
-1.633^{* * *} \\
{[0.481]}\end{array}$ & $\begin{array}{c}-0.00682^{* * *} \\
{[0.00266]} \\
-1.608^{* * *} \\
{[0.527]}\end{array}$ & $\begin{array}{c}-0.00702 * * * \\
{[0.00211]} \\
-1.571^{* * *} \\
{[0.493]}\end{array}$ & $\begin{array}{c}-0.00585^{* *} \\
{[0.00245]} \\
-1.463^{* * *} \\
{[0.511]}\end{array}$ & $\begin{array}{c}-0.00610^{* * *} \\
{[0.00230]} \\
-1.401 * * \\
{[0.557]}\end{array}$ \\
\hline $\begin{array}{l}\text { F-stat (1 } 1^{\text {st }} \text { stage) } \\
\text { Prob J-Stat }\end{array}$ & & & & & & & & & $\begin{array}{c}13.126 \\
0.010\end{array}$ & $\begin{array}{c}10.072 \\
0.004\end{array}$ & $\begin{array}{c}14.403 \\
0.010\end{array}$ & $\begin{array}{l}8.658 \\
0.010\end{array}$ & $\begin{array}{l}8.043 \\
0.004\end{array}$ \\
\hline
\end{tabular}

Note: The explanatory variables except the dummies are in logarithm. The aerial distances are measured in kilometers. Robust standard errors are reported in brackets. ${ }^{* * *}$ indicates significance

at the $1 \%$-level, $* *$ indicates significance at the $5 \%$-level, * indicates significance at the $10 \%$-level. 
Table D.2: The effect of industrialization on spending by communes, 1855-1863

\begin{tabular}{|c|c|c|c|c|c|c|c|c|c|c|c|c|}
\hline & (1) & $(2)$ & (3) & (4) & (5) & (6) & $(7)$ & (8) & (9) & (10) & (11) & (12) \\
\hline & OLS & OLS & OLS & OLS & OLS & OLS & OLS & IV & IV & IV & IV & IV \\
\hline & \multicolumn{12}{|c|}{ Commune Spending on Primary Schooling per Inhabitant, 1855-1863 } \\
\hline \multirow[t]{2}{*}{ Number of Steam Engines } & 0.0145 & -0.0117 & -0.00446 & -0.00267 & 0.00319 & 0.00308 & -0.000178 & $0.0496^{* *}$ & $0.0331^{*}$ & $0.0515^{* * *}$ & $0.0718^{* *}$ & $0.0435^{* *}$ \\
\hline & {$[0.0112]$} & {$[0.0143]$} & {$[0.0132]$} & {$[0.00959]$} & {$[0.0121]$} & {$[0.0116]$} & {$[0.0102]$} & {$[0.0209]$} & {$[0.0177]$} & {$[0.0186]$} & {$[0.0281]$} & {$[0.0210]$} \\
\hline \multirow[t]{2}{*}{ Average Rainfall } & & 0.105 & $0.204^{* *}$ & 0.101 & $0.188^{* *}$ & 0.151 & 0.0823 & $0.261^{* * *}$ & $0.156^{* *}$ & $0.226^{* * *}$ & 0.150 & 0.0993 \\
\hline & & {$[0.0957]$} & {$[0.0898]$} & {$[0.0890]$} & {$[0.0912]$} & {$[0.103]$} & {$[0.0960]$} & {$[0.0848]$} & {$[0.0787]$} & {$[0.0862]$} & {$[0.105]$} & {$[0.0910]$} \\
\hline \multirow{2}{*}{ Average Temperature } & & $-0.386 * * *$ & -0.142 & 0.143 & -0.106 & -0.159 & 0.138 & -0.0998 & 0.171 & -0.0527 & -0.132 & 0.155 \\
\hline & & {$[0.139]$} & {$[0.120]$} & {$[0.127]$} & {$[0.116]$} & {$[0.118]$} & {$[0.125]$} & {$[0.125]$} & {$[0.123]$} & {$[0.116]$} & {$[0.120]$} & {$[0.115]$} \\
\hline \multirow[t]{2}{*}{ Latitude } & & 0.435 & -0.593 & $-1.091^{*}$ & -0.661 & -0.622 & $-1.134^{*}$ & -0.846 & $-1.157^{*}$ & -0.905 & -0.936 & $-1.362^{* *}$ \\
\hline & & {$[0.326]$} & {$[0.613]$} & {$[0.639]$} & {$[0.686]$} & {$[0.641]$} & {$[0.673]$} & {$[0.656]$} & {$[0.655]$} & {$[0.751]$} & {$[0.738]$} & {$[0.681]$} \\
\hline \multirow[t]{2}{*}{ Land Suitability } & & $0.266^{* * *}$ & $0.173^{* *}$ & 0.0939 & $0.175^{* *}$ & $0.176^{* * *}$ & 0.0945 & $0.155^{* *}$ & 0.0845 & $0.161^{* *}$ & $0.160^{* * *}$ & $0.0890^{*}$ \\
\hline & & {$[0.0808]$} & {$[0.0696]$} & {$[0.0639]$} & {$[0.0680]$} & {$[0.0663]$} & {$[0.0626]$} & {$[0.0663]$} & {$[0.0593]$} & {$[0.0632]$} & {$[0.0598]$} & {$[0.0529]$} \\
\hline \multirow[t]{2}{*}{ Share of Carboniferous Area } & & & $-0.228^{* *}$ & $-0.185^{* *}$ & $-0.256^{* * *}$ & $-0.232^{* *}$ & $-0.185^{* *}$ & $-0.243 * * *$ & $-0.197^{* * *}$ & $-0.282^{* * *}$ & $-0.253^{* * *}$ & $-0.204^{* * *}$ \\
\hline & & & {$[0.0927]$} & {$[0.0772]$} & {$[0.0905]$} & {$[0.0937]$} & {$[0.0789]$} & {$[0.0799]$} & {$[0.0646]$} & {$[0.0788]$} & {$[0.0913]$} & {$[0.0678]$} \\
\hline \multirow{2}{*}{ Maritime Department } & & & -0.0536 & $-0.0869^{* *}$ & -0.0574 & -0.0463 & $-0.0831^{* *}$ & $-0.0936^{* *}$ & $-0.117 * * *$ & $-0.0922^{* *}$ & $-0.0815^{*}$ & $-0.103^{* *}$ \\
\hline & & & {$[0.0333]$} & {$[0.0338]$} & {$[0.0359]$} & {$[0.0343]$} & {$[0.0354]$} & {$[0.0402]$} & {$[0.0408]$} & {$[0.0421]$} & {$[0.0433]$} & {$[0.0404]$} \\
\hline Border Department & & & $\begin{array}{l}0.0579^{*} \\
{[0.0327]}\end{array}$ & $\begin{array}{l}0.00973 \\
{[0.0330]}\end{array}$ & $\begin{array}{l}0.0659^{*} \\
{[0.0340]}\end{array}$ & $\begin{array}{c}0.0530 \\
{[0.0330]}\end{array}$ & $\begin{array}{l}0.00791 \\
{[0.0351]}\end{array}$ & $\begin{array}{c}0.0461 \\
{[0.0367]}\end{array}$ & $\begin{array}{l}0.00835 \\
{[0.0334]}\end{array}$ & $\begin{array}{l}0.0604^{*} \\
{[0.0354]}\end{array}$ & 0.0341 & 0.00724 \\
\hline \multirow{2}{*}{ Distance to Paris } & & & $-0.000403^{* *}$ & $-0.000422^{* *}$ & $-0.000395^{* *}$ & $\begin{array}{c}{[0.0330]} \\
-0.000388^{* *}\end{array}$ & $\begin{array}{c}{[0.0351]} \\
-0.000424^{* *}\end{array}$ & $\begin{array}{c}{[0.0367]} \\
-0.000374^{*}\end{array}$ & $\begin{array}{c}{[0.0334]} \\
-0.000392^{* *}\end{array}$ & $\begin{array}{c}{[0.0354]} \\
-0.000367^{*}\end{array}$ & $\begin{array}{c}{[0.0398]} \\
-0.000338\end{array}$ & $-0.000414^{* *}$ \\
\hline & & & {$[0.000180]$} & {$[0.000185]$} & {$[0.000195]$} & {$[0.000189]$} & {$[0.000193]$} & {$[0.000200]$} & {$[0.000191]$} & {$[0.000217]$} & {$[0.000227]$} & [0.000201] \\
\hline \multirow[t]{2}{*}{ Paris and Suburbs } & & & -0.0956 & 0.0376 & -0.0914 & -0.0894 & 0.0322 & -0.114 & 0.0465 & -0.104 & -0.102 & 0.0265 \\
\hline & & & {$[0.182]$} & {$[0.0414]$} & {$[0.167]$} & {$[0.166]$} & {$[0.0418]$} & {$[0.192]$} & {$[0.0581]$} & {$[0.165]$} & {$[0.160]$} & {$[0.0543]$} \\
\hline Grooms who Signed their Marriage License, $1786-1790$ & & & & $0.306^{* * *}$ & & & $\begin{array}{l}0.308^{* * *} \\
{[0.0644]}\end{array}$ & & $0.289^{* * *}$ & & & $\begin{array}{l}0.284^{* * *} \\
{[0.0605]}\end{array}$ \\
\hline \multirow{2}{*}{ University } & & & & {$[0.0618]$} & $-0.0694^{*}$ & & $\begin{array}{l}{[0.0044]} \\
-0.00119\end{array}$ & & {$[0.0622]$} & $-0.106^{* * *}$ & & $\begin{array}{l}{[0.0605]} \\
-0.0280\end{array}$ \\
\hline & & & & & {$[0.0362]$} & & {$[0.0274]$} & & & {$[0.0372]$} & & {$[0.0247]$} \\
\hline \multirow[t]{2}{*}{ Urban Population in 1700} & & & & & & -0.0135 & -0.00457 & & & & $-0.0301^{* *}$ & -0.0134 \\
\hline & & & & & & {$[0.0109]$} & {$[0.00798]$} & & & & {$[0.0139]$} & {$[0.00929]$} \\
\hline Adjusted R2 & 0.007 & 0.297 & 0.386 & 0.650 & 0.412 & 0.397 & 0.642 & & & & & \\
\hline \multirow[t]{2}{*}{ Observations } & 85 & 85 & 85 & 79 & 85 & 85 & 79 & 85 & 79 & 85 & 85 & 79 \\
\hline & \multicolumn{12}{|c|}{ First stage: the instrumented variable is Number of Steam Engines } \\
\hline \multirow{2}{*}{$\begin{array}{l}\text { Distance to Fresnes } \\
\text { Deviation from Wheat Prices in 1835-1838 } \\
\text { (baseline 1810-1829) }\end{array}$} & & & & & & & & $\begin{array}{c}-0.00687^{* * *} \\
{[0.00250]}\end{array}$ & $\begin{array}{c}-0.00682^{* *} \\
{[0.00266]}\end{array}$ & $\begin{array}{c}-0.00702^{* * *} \\
{[0.00211]}\end{array}$ & $\begin{array}{c}-0.00585^{* *} \\
{[0.00245]}\end{array}$ & $\begin{array}{c}-0.00610^{* * *} \\
{[0.00230]}\end{array}$ \\
\hline & & & & & & & & $\begin{array}{c}-1.633^{* * *} \\
{[0.481]}\end{array}$ & $\begin{array}{c}-1.608^{* * *} \\
{[0.527]}\end{array}$ & $\begin{array}{c}-1.571^{* * *} \\
{[0.493]}\end{array}$ & $\begin{array}{c}-1.463^{* * *} \\
{[0.511]}\end{array}$ & $\begin{array}{c}-1.401^{* *} \\
{[0.557]}\end{array}$ \\
\hline F-stat ( $1^{s t}$ stage $)$ & & & & & & & & 13.126 & 10.072 & 14.403 & 8.658 & 8.043 \\
\hline Prob J-Stat & & & & & & & & 0.409 & 0.401 & 0.469 & 0.381 & 0.412 \\
\hline
\end{tabular}

Note: The explanatory variables except the dummies are in logarithm. The aerial distances are measured in kilometers. Robust standard errors are reported in brackets. ${ }^{* * *}$ indicates significance

at the $1 \%$-level, $* *$ indicates significance at the $5 \%$-level, * indicates significance at the $10 \%$-level. 
Table D.3: The effect of industrialization on spending by departments, 1855-1863

\begin{tabular}{|c|c|c|c|c|c|c|c|c|c|c|c|c|}
\hline & (1) & (2) & (3) & (4) & (5) & (6) & (7) & $(8)$ & (9) & (10) & (11) & (12) \\
\hline & OLS & OLS & OLS & OLS & OLS & OLS & OLS & IV & IV & IV & IV & IV \\
\hline & \multicolumn{12}{|c|}{ Department Spending on Primary Schooling per Inhabitant, 1855-1863 } \\
\hline \multirow[t]{2}{*}{ Number of Steam Engines } & $0.0126^{* * *}$ & 0.00559 & 0.00580 & 0.00689 & $0.00731^{*}$ & 0.00541 & 0.00674 & $0.0153^{*}$ & 0.0160 & $0.0160^{*}$ & 0.0163 & 0.0164 \\
\hline & {$[0.00443]$} & {$[0.00484]$} & {$[0.00439]$} & {$[0.00478]$} & {$[0.00423]$} & {$[0.00446]$} & {$[0.00481]$} & {$[0.00853]$} & {$[0.0100]$} & {$[0.00854]$} & {$[0.00999]$} & {$[0.0111]$} \\
\hline \multirow{2}{*}{ Average Rainfall } & & 0.00873 & 0.0228 & 0.00711 & 0.0196 & 0.0255 & 0.0189 & 0.0327 & 0.0211 & 0.0266 & 0.0253 & 0.0227 \\
\hline & & {$[0.0242]$} & {$[0.0269]$} & {$[0.0312]$} & {$[0.0293]$} & {$[0.0284]$} & {$[0.0348]$} & {$[0.0257]$} & {$[0.0298]$} & {$[0.0281]$} & {$[0.0269]$} & {$[0.0317]$} \\
\hline \multirow[t]{2}{*}{ Average Temperature } & & -0.0104 & -0.0491 & -0.0364 & -0.0420 & -0.0483 & -0.0342 & -0.0417 & -0.0293 & -0.0324 & -0.0440 & -0.0305 \\
\hline & & {$[0.0247]$} & {$[0.0483]$} & {$[0.0557]$} & {$[0.0470]$} & {$[0.0484]$} & {$[0.0555]$} & {$[0.0413]$} & {$[0.0465]$} & {$[0.0394]$} & {$[0.0428]$} & {$[0.0477]$} \\
\hline \multirow[t]{2}{*}{ Latitude } & & $0.303^{* *}$ & 0.221 & 0.0714 & 0.207 & 0.222 & 0.0540 & 0.176 & 0.0545 & 0.163 & 0.172 & 0.00350 \\
\hline & & {$[0.117]$} & {$[0.218]$} & {$[0.253]$} & {$[0.228]$} & {$[0.216]$} & {$[0.249]$} & {$[0.207]$} & {$[0.239]$} & {$[0.221]$} & {$[0.214]$} & {$[0.234]$} \\
\hline \multirow[t]{2}{*}{ Land Suitability } & & $0.0502^{* * * *}$ & $0.0487^{* * *}$ & $0.0362^{* *}$ & $0.0490^{* * * *}$ & $0.0485^{* * *}$ & $0.0375^{* *}$ & $0.0455^{* * *}$ & $0.0338^{* *}$ & $0.0464^{* * *}$ & $0.0459^{* * *}$ & $0.0363^{* * *}$ \\
\hline & & {$[0.0159]$} & {$[0.0160]$} & {$[0.0159]$} & {$[0.0158]$} & {$[0.0161]$} & {$[0.0165]$} & {$[0.0140]$} & {$[0.0131]$} & {$[0.0138]$} & {$[0.0142]$} & {$[0.0139]$} \\
\hline \multirow[t]{2}{*}{ Share of Carboniferous Area } & & & $-0.0430^{*}$ & -0.0271 & $-0.0484^{* *}$ & $-0.0427^{*}$ & -0.0335 & $-0.0456^{* *}$ & -0.0301 & $-0.0533^{* *}$ & $-0.0462^{* *}$ & $-0.0378^{*}$ \\
\hline & & & {$[0.0228]$} & {$[0.0259]$} & {$[0.0231]$} & {$[0.0230]$} & {$[0.0260]$} & {$[0.0196]$} & {$[0.0208]$} & {$[0.0209]$} & {$[0.0204]$} & {$[0.0203]$} \\
\hline \multirow[t]{2}{*}{ Maritime Department } & & & 0.00759 & 0.0117 & 0.00683 & 0.00721 & 0.0105 & 0.000576 & 0.00408 & 0.000545 & 0.00161 & 0.00605 \\
\hline & & & {$[0.0177]$} & {$[0.0206]$} & {$[0.0175]$} & {$[0.0175]$} & {$[0.0202]$} & {$[0.0153]$} & {$[0.0176]$} & {$[0.0150]$} & {$[0.0154]$} & {$[0.0172]$} \\
\hline Border Department & & & $\begin{array}{l}-0.0269^{* *} \\
{[0.0122]}\end{array}$ & $\begin{array}{l}-0.0277^{*} \\
{[0.0144]}\end{array}$ & $\begin{array}{c}-0.0253^{* *} \\
{[0.0122]}\end{array}$ & $\begin{array}{c}-0.0267 * * \\
{[0.0124]}\end{array}$ & $\begin{array}{l}-0.0239^{*} \\
{[0.0140]}\end{array}$ & $\begin{array}{c}-0.0290^{* *} \\
{[0.0118]}\end{array}$ & $\begin{array}{c}-0.0281^{* *} \\
{[0.0139]}\end{array}$ & $\begin{array}{c}-0.0263^{* *} \\
{[0.0114]}\end{array}$ & $\begin{array}{c}-0.0297^{* *} \\
{[0.0127]}\end{array}$ & $\begin{array}{l}-0.0240^{*} \\
{[0.0133]}\end{array}$ \\
\hline \multirow[t]{2}{*}{ Distance to Paris } & & & $-1.67 \mathrm{e}-05$ & $-4.78 \mathrm{e}-05$ & $-1.52 \mathrm{e}-05$ & $-1.75 \mathrm{e}-05$ & $-5.49 \mathrm{e}-05$ & $\begin{array}{l}{[0.0118]} \\
-1.16 \mathrm{e}-05\end{array}$ & $-4.01 \mathrm{e}-05$ & $\begin{array}{l}{[0.0114]} \\
-1.01 \mathrm{e}-05\end{array}$ & $-9.49 \mathrm{e}-06$ & $\begin{array}{l}-5.25 \mathrm{e}-05 \\
-5.05]\end{array}$ \\
\hline & & & {$[6.50 \mathrm{e}-05]$} & [7.31e-05] & {$[6.80 \mathrm{e}-05]$} & [6.56e--05] & [7.30e-05] & [6.37e--05] & [7.04e-05] & [6.73e--05] & [6.62e--05] & {$[6.90 \mathrm{e}-05]$} \\
\hline Paris and Suburbs & & & $\begin{array}{l}-0.0212 \\
{[0.0395]}\end{array}$ & $\begin{array}{l}0.0134 \\
{[0.0167]}\end{array}$ & $\begin{array}{l}-0.0204 \\
{[0.0365]}\end{array}$ & $\begin{array}{l}-0.0215 \\
{[0.0403]}\end{array}$ & $\begin{array}{c}0.0145 \\
{[0.0199]}\end{array}$ & $\begin{array}{l}-0.0244 \\
{[0.0405]}\end{array}$ & $\begin{array}{l}0.0157 \\
{[0.0186]}\end{array}$ & $\begin{array}{l}-0.0227 \\
{[0.0356]}\end{array}$ & $\begin{array}{l}-0.0236 \\
{[0.0382]}\end{array}$ & $\begin{array}{l}0.0133 \\
{[0.0203]}\end{array}$ \\
\hline Grooms who Signed their Marriage License, $1786-1790$ & & & & $\begin{array}{c}0.0244 \\
{[0.0268]}\end{array}$ & & & $\begin{array}{c}0.0161 \\
{[0.0264]}\end{array}$ & & $\begin{array}{c}0.0199 \\
{[0.0261]}\end{array}$ & & & $\begin{array}{c}0.0106 \\
{[0.0257]}\end{array}$ \\
\hline \multirow[t]{2}{*}{ University } & & & & & -0.0137 & & -0.0141 & & & -0.0202 & & -0.0200 \\
\hline & & & & & {$[0.0126]$} & & {$[0.0143]$} & & & {$[0.0147]$} & & {$[0.0163]$} \\
\hline Urban Population in 1700 & & & & & & $\begin{array}{l}0.000706 \\
{[0.00297]}\end{array}$ & $\begin{array}{c}0.00327 \\
{[0.00283]}\end{array}$ & & & & $\begin{array}{l}-0.00192 \\
{[0.00377]}\end{array}$ & $\begin{array}{c}0.00132 \\
{[0.00308]}\end{array}$ \\
\hline Adjusted R2 & 0.121 & 0.253 & 0.267 & 0.315 & 0.269 & 0.257 & 0.310 & & & & & \\
\hline \multirow[t]{2}{*}{ Observations } & 85 & 85 & 85 & 79 & 85 & 85 & 79 & 85 & 79 & 85 & 85 & 79 \\
\hline & \multicolumn{12}{|c|}{ First stage: the instrumented variable is Number of Steam Engines } \\
\hline \multirow{2}{*}{$\begin{array}{l}\text { Distance to Fresnes } \\
\text { Deviation from Wheat Prices in 1835-1838 } \\
\text { (baseline 1810-1829) }\end{array}$} & & & & & & & & $\begin{array}{c}-0.00687^{* * *} \\
{[0.00250]}\end{array}$ & $\begin{array}{c}-0.00682^{* *} \\
{[0.00266]}\end{array}$ & $\begin{array}{c}-0.00702^{* * *} \\
{[0.00211]}\end{array}$ & $\begin{array}{c}-0.00585^{* *} \\
{[0.00245]}\end{array}$ & $\begin{array}{c}-0.00610^{* * *} \\
{[0.00230]}\end{array}$ \\
\hline & & & & & & & & $\begin{array}{c}-1.633^{* * *} \\
{[0.481]}\end{array}$ & $\begin{array}{c}-1.608^{* * *} \\
{[0.527]}\end{array}$ & $\begin{array}{c}-1.571^{* * *} \\
{[0.493]}\end{array}$ & $\begin{array}{c}-1.463^{* * *} \\
{[0.511]}\end{array}$ & $\begin{array}{c}-1.401^{* *} \\
{[0.557]}\end{array}$ \\
\hline F-stat ( $1^{s t}$ stage $)$ & & & & & & & & 13.126 & 10.072 & 14.403 & 8.658 & 8.043 \\
\hline Prob J-Stat & & & & & & & & 0.084 & 0.116 & 0.078 & 0.084 & 0.112 \\
\hline
\end{tabular}

Note: The explanatory variables except the dummies are in logarithm. The aerial distances are measured in kilometers. Robust standard errors are reported in brackets. ${ }^{* * *}$ indicates significance at the $1 \%$-level, ** indicates significance at the $5 \%$-level, * indicates significance at the $10 \%$-level. 
Table D.4: The effect of industrialization on spending by the central state, 1855-1863

\begin{tabular}{|c|c|c|c|c|c|c|c|c|c|c|c|c|}
\hline & $\begin{array}{l}\text { (1) } \\
\text { OLS }\end{array}$ & $\begin{array}{l}(2) \\
\text { OLS }\end{array}$ & $\begin{array}{l}\text { OLS } \\
\text { OLS }\end{array}$ & $\begin{array}{c}(4) \\
\text { OLS }\end{array}$ & $\begin{array}{l}(5) \\
\text { OLS }\end{array}$ & $\begin{array}{c}(6) \\
\text { OLS }\end{array}$ & $\begin{array}{c}(7) \\
\text { OLS }\end{array}$ & $\begin{array}{l}(8) \\
\text { IV }\end{array}$ & $\begin{array}{l}(9) \\
\text { IV }\end{array}$ & $\begin{array}{l}\text { (10) } \\
\text { IV }\end{array}$ & $\begin{array}{l}\text { (11) } \\
\text { IV }\end{array}$ & $\begin{array}{l}(12) \\
\text { IV }\end{array}$ \\
\hline & \multicolumn{12}{|c|}{ Central State Spending on Primary Schooling per Inhabitant, 1855-1863 } \\
\hline Number of Steam Engines & $\begin{array}{c}-0.0495^{* * *} \\
{[0.0111]}\end{array}$ & $\begin{array}{c}-0.0304^{* * *} \\
{[0.00969]}\end{array}$ & $\begin{array}{c}-0.0353^{* * *} \\
{[0.0103]}\end{array}$ & $\begin{array}{c}-0.0365^{* * *} \\
{[0.0112]}\end{array}$ & $\begin{array}{c}-0.0322^{* * *} * \\
{[0.0108]}\end{array}$ & $\begin{array}{c}-0.0273^{* *} \\
{[0.0104]}\end{array}$ & $\begin{array}{c}-0.0242^{* *} \\
{[0.0119]}\end{array}$ & $\begin{array}{c}-0.0440 * * * \\
{[0.0152]}\end{array}$ & $\begin{array}{c}-0.0519^{* * *} \\
{[0.0178]}\end{array}$ & $\begin{array}{c}-0.0426^{* * *} \\
{[0.0154]}\end{array}$ & $\begin{array}{c}-0.0356^{* *} \\
{[0.0163]}\end{array}$ & $\begin{array}{c}-0.0406^{* *} \\
{[0.0187]}\end{array}$ \\
\hline Average Rainfall & & $\begin{array}{l}-0.0382 \\
{[0.0917]}\end{array}$ & $\begin{array}{l}-0.0245 \\
{[0.104]}\end{array}$ & $\begin{array}{l}-0.0759 \\
{[0.114]}\end{array}$ & $\begin{array}{r}-0.0309 \\
{[0.104]}\end{array}$ & $\begin{array}{l}-0.0799 \\
{[0.106]}\end{array}$ & $\begin{array}{l}-0.155 \\
{[0.116]}\end{array}$ & $\begin{array}{l}-0.0336 \\
{[0.0940]}\end{array}$ & $\begin{array}{l}-0.0996 \\
{[0.0989]}\end{array}$ & $\begin{array}{l}-0.0392 \\
{[0.0933]}\end{array}$ & $\begin{array}{l}-0.0797 \\
{[0.0983]}\end{array}$ & $\begin{array}{l}-0.161 \\
{[0.101]}\end{array}$ \\
\hline Average Temperature & & $\begin{array}{c}-0.470^{* * *} \\
{[0.125]}\end{array}$ & $\begin{array}{c}-0.473^{* * *} \\
{[0.145]}\end{array}$ & $\begin{array}{c}-0.423^{* * *} \\
{[0.157]}\end{array}$ & $\begin{array}{c}-0.458^{* * *} \\
{[0.151]}\end{array}$ & $\begin{array}{c}-0.490 * * * \\
{[0.140]}\end{array}$ & $\begin{array}{c}-0.445 * * * \\
{[0.143]}\end{array}$ & $\begin{array}{c}-0.480^{* * *} \\
{[0.131]}\end{array}$ & $\begin{array}{c}-0.435^{* * *} \\
{[0.135]}\end{array}$ & $\begin{array}{c}-0.470^{* * *} \\
{[0.134]}\end{array}$ & $\begin{array}{c}-0.493^{* * *} \\
{[0.126]}\end{array}$ & $\begin{array}{c}-0.451^{* * *} \\
{[0.124]}\end{array}$ \\
\hline Latitude & & $\begin{array}{c}-1.349^{* * *} \\
{[0.295]}\end{array}$ & $\begin{array}{c}-1.649^{* * *} \\
{[0.569]}\end{array}$ & $\begin{array}{c}-1.689^{* *} \\
{[0.664]}\end{array}$ & $\begin{array}{c}-1.676^{* * *} \\
{[0.556]}\end{array}$ & $\begin{array}{c}-1.679 * * * \\
{[0.574]}\end{array}$ & $\begin{array}{c}-1.923^{* * *} \\
{[0.677]}\end{array}$ & $\begin{array}{c}-1.608 * * * \\
{[0.537]}\end{array}$ & $\begin{array}{c}-1.660^{* * *} \\
{[0.640]}\end{array}$ & $\begin{array}{c}-1.624^{* * *} \\
{[0.526]}\end{array}$ & $\begin{array}{c}-1.641^{* * *} \\
{[0.534]}\end{array}$ & $\begin{array}{c}-1.838^{* * *} \\
{[0.650]}\end{array}$ \\
\hline Land Suitability & & $\begin{array}{l}-0.0389 \\
{[0.0514]}\end{array}$ & $\begin{array}{l}-0.0649 \\
{[0.0399]}\end{array}$ & $\begin{array}{c}-0.0951^{* *} \\
{[0.0453]}\end{array}$ & $\begin{array}{c}-0.0643^{*} \\
{[0.0380]}\end{array}$ & $\begin{array}{c}-0.0618^{*} \\
{[0.0363]}\end{array}$ & $\begin{array}{c}-0.0905^{* *} \\
{[0.0394]}\end{array}$ & $\begin{array}{l}-0.0620 \\
{[0.0384]}\end{array}$ & $\begin{array}{c}-0.0910^{* *} \\
{[0.0422]}\end{array}$ & $\begin{array}{c}-0.0613^{*} \\
{[0.0370]}\end{array}$ & $\begin{array}{c}-0.0598^{*} \\
{[0.0348]}\end{array}$ & $\begin{array}{c}-0.0884^{* *} \\
{[0.0372]}\end{array}$ \\
\hline Share of Carboniferous Area & & & $\begin{array}{l}-0.0943 \\
{[0.0779]}\end{array}$ & $\begin{array}{l}-0.0428 \\
{[0.0921]}\end{array}$ & $\begin{array}{c}-0.106 \\
{[0.0806]}\end{array}$ & $\begin{array}{l}-0.0986 \\
{[0.0758]}\end{array}$ & $\begin{array}{l}-0.0461 \\
{[0.0934]}\end{array}$ & $\begin{array}{l}-0.0919 \\
{[0.0712]}\end{array}$ & $\begin{array}{l}-0.0377 \\
{[0.0800]}\end{array}$ & $\begin{array}{l}-0.0997 \\
{[0.0729]}\end{array}$ & $\begin{array}{l}-0.0960 \\
{[0.0684]}\end{array}$ & $\begin{array}{l}-0.0387 \\
{[0.0786]}\end{array}$ \\
\hline Maritime Department & & & $\begin{array}{c}0.0430 \\
{[0.0283]}\end{array}$ & $\begin{array}{c}0.0428 \\
{[0.0299]}\end{array}$ & $\begin{array}{c}0.0415 \\
{[0.0285]}\end{array}$ & $\begin{array}{l}0.0507^{*} \\
{[0.0284]}\end{array}$ & $\begin{array}{c}0.0607^{* *} \\
{[0.0289]}\end{array}$ & $\begin{array}{l}0.0495^{*} \\
{[0.0289]}\end{array}$ & $\begin{array}{l}0.0557^{*} \\
{[0.0312]}\end{array}$ & $\begin{array}{l}0.0490^{*} \\
{[0.0285]}\end{array}$ & $\begin{array}{c}0.0550^{* *} \\
{[0.0280]}\end{array}$ & $\begin{array}{l}0.0682^{2 * *} \\
{[0.0277}\end{array}$ \\
\hline Border Department & & & $\begin{array}{c}0.0351 \\
{[0.0367]}\end{array}$ & $\begin{array}{c}0.0324 \\
{[0.0376]}\end{array}$ & $\begin{array}{c}0.0383 \\
{[0.0367]}\end{array}$ & $\begin{array}{c}0.0299 \\
{[0.0349]}\end{array}$ & $\begin{array}{c}0.0280 \\
{[0.0363]}\end{array}$ & $\begin{array}{c}0.0370 \\
{[0.0343]}\end{array}$ & $\begin{array}{c}0.0330 \\
{[0.0353]}\end{array}$ & $\begin{array}{c}0.0395 \\
{[0.0343]}\end{array}$ & $\begin{array}{c}0.0322 \\
{[0.0327]}\end{array}$ & $\begin{array}{c}0.0282 \\
{[0.0341]}\end{array}$ \\
\hline Distance to Paris & & & $\begin{array}{l}-0.000101 \\
{[0.000172]}\end{array}$ & $\begin{array}{l}-8.27 \mathrm{e}-05 \\
{[0.000184]}\end{array}$ & $\begin{array}{c}-9.75 \mathrm{e}-05 \\
{[0.000168]}\end{array}$ & $\begin{array}{c}-8.50 \mathrm{e}-05 \\
{[0.000168]}\end{array}$ & $\begin{array}{l}-0.000104 \\
{[0.000179]}\end{array}$ & $\begin{array}{l}-0.000105 \\
{[0.000163]}\end{array}$ & $\begin{array}{c}-9.57 \mathrm{e}-05 \\
{[0.000177]}\end{array}$ & $\begin{array}{c}-0.000104 \\
{[0.000159]}\end{array}$ & $\begin{array}{c}-9.11 \mathrm{e}-05 \\
{[0.000158]}\end{array}$ & $\begin{array}{c}-0.000108 \\
{[0.000172]}\end{array}$ \\
\hline Paris and Suburbs & & & $\begin{array}{c}0.0106 \\
{[0.0352]}\end{array}$ & $\begin{array}{l}-0.0247 \\
{[0.0396]}\end{array}$ & $\begin{array}{c}0.0123 \\
{[0.0379]}\end{array}$ & $\begin{array}{c}0.0171 \\
{[0.0492]}\end{array}$ & $\begin{array}{l}-0.0500 \\
{[0.0502]}\end{array}$ & $\begin{array}{l}0.0136 \\
{[0.0375]}\end{array}$ & $\begin{array}{l}-0.0285 \\
{[0.0432]}\end{array}$ & $\begin{array}{l}0.0151 \\
{[0.0391]}\end{array}$ & $\begin{array}{c}0.0186 \\
{[0.0473]}\end{array}$ & $\begin{array}{l}-0.0478 \\
{[0.0514]}\end{array}$ \\
\hline Grooms who Signed their Marriage License, $1786-1790$ & & & & $\begin{array}{c}0.0863 \\
{[0.0676]}\end{array}$ & & & $\begin{array}{c}0.0867 \\
{[0.0684]}\end{array}$ & & $\begin{array}{c}0.0938 \\
{[0.0602]}\end{array}$ & & & $\begin{array}{c}0.0960 \\
{[0.0604]}\end{array}$ \\
\hline University & & & & & $\begin{array}{l}-0.0280 \\
{[0.0216]}\end{array}$ & & $\begin{array}{l}-0.0228 \\
{[0.0213]}\end{array}$ & & & $\begin{array}{l}-0.0202 \\
{[0.0213]}\end{array}$ & & $\begin{array}{l}-0.0127 \\
{[0.0211]}\end{array}$ \\
\hline Urban Population in 1700 & & & & & & $\begin{array}{c}-0.0143^{* *} \\
{[0.00617]}\end{array}$ & $\begin{array}{c}-0.0189^{* *} \\
{[0.00725]}\end{array}$ & & & & $\begin{array}{l}-0.0123^{*} \\
{[0.00679]}\end{array}$ & $\begin{array}{c}-0.0156^{* *} \\
{[0.00734]}\end{array}$ \\
\hline Adjusted R2 & 0.243 & 0.560 & 0.568 & 0.573 & 0.568 & 0.587 & 0.607 & & & & & \\
\hline Observations & 85 & 85 & 85 & 79 & 85 & 85 & 79 & 85 & 79 & 85 & 85 & 79 \\
\hline & & & & First & age: the is & trumented v & ariable is 1 & ber of Ste & Engines & & & \\
\hline Distance to Fresnes & & & & & & & & $\begin{array}{c}-0.00687^{* * *} \\
{[0.00250]}\end{array}$ & $\begin{array}{c}-0.00682^{* *} \\
{[0.00266]}\end{array}$ & $\begin{array}{c}-0.00702^{* * *} \\
{[0.00211]}\end{array}$ & $\begin{array}{c}-0.00585^{* *} \\
{[0.00245]}\end{array}$ & $\begin{array}{c}-0.00610^{* * *} \\
{[0.00230]}\end{array}$ \\
\hline $\begin{array}{l}\text { Deviation from Wheat Prices in 1835-1838 } \\
\text { (baseline 1810-1829) }\end{array}$ & & & & & & & & $\begin{array}{c}-1.633^{* * *} \\
{[0.481]}\end{array}$ & $\begin{array}{c}-1.608 * * * \\
{[0.527]}\end{array}$ & $\begin{array}{c}-1.571^{* * *} \\
{[0.493]}\end{array}$ & $\begin{array}{c}-1.463^{* * *} \\
{[0.511]}\end{array}$ & $\begin{array}{c}-1.401^{* *} \\
{[0.557]}\end{array}$ \\
\hline F-stat ( $1^{\text {st }}$ stage $)$ & & & & & & & & 13.126 & 10.072 & 14.403 & 8.658 & 8.043 \\
\hline Prob J-Stat & & & & & & & & 0.005 & 0.007 & 0.006 & 0.003 & 0.005 \\
\hline
\end{tabular}

Note: The explanatory variables except the dummies are in logarithm. The aerial distances are measured in kilometers. Robust standard errors are reported in brackets. ${ }^{* * *}$ indicates significance at the $1 \%$-level, $* *$ indicates significance at the $5 \%$-level, * indicates significance at the $10 \%$-level. 
Table D.5: The effect of industrialization on the number of school buildings in 1850

\begin{tabular}{|c|c|c|c|c|c|c|c|c|c|c|c|c|}
\hline & (1) & $(2)$ & (3) & (4) & (5) & $\begin{array}{ll}(6) \\
\end{array}$ & $\begin{array}{c}(7) \\
\end{array}$ & (8) & $(9)$ & (10) & $\begin{array}{c}(11) \\
\end{array}$ & $\begin{array}{l}(12) \\
\end{array}$ \\
\hline & & \multicolumn{11}{|c|}{ Schools per commune, 1850} \\
\hline Number of Steam Engines & $\begin{array}{c}0.0527 \\
{[0.0351]}\end{array}$ & $\begin{array}{c}0.138^{* * *} \\
{[0.0382]}\end{array}$ & $\begin{array}{c}0.125^{* * *} \\
{[0.0365]}\end{array}$ & $\begin{array}{c}0.0932^{* * *} \\
{[0.0320]}\end{array}$ & $\begin{array}{c}0.111^{* * *} \\
{[0.0376]}\end{array}$ & $\begin{array}{c}0.0970^{* * *} \\
{[0.0325]}\end{array}$ & $\begin{array}{c}0.0840^{* *} \\
{[0.0369]}\end{array}$ & $\begin{array}{c}0.167^{* * *} \\
{[0.0595]}\end{array}$ & $\begin{array}{l}0.147^{* *} \\
{[0.0612]}\end{array}$ & $\begin{array}{c}0.165^{* * *} \\
{[0.0612]}\end{array}$ & $\begin{array}{c}0.136^{*} \\
{[0.0702]}\end{array}$ & $\begin{array}{l}0.147^{* *} \\
{[0.0709]}\end{array}$ \\
\hline Average Rainfall & & $\begin{array}{l}-0.0325 \\
{[0.275]}\end{array}$ & $\begin{array}{l}-0.128 \\
{[0.235]}\end{array}$ & $\begin{array}{l}-0.261 \\
{[0.284]}\end{array}$ & $\begin{array}{l}-0.0981 \\
{[0.260]}\end{array}$ & $\begin{array}{l}0.0695 \\
{[0.2671}\end{array}$ & $\begin{array}{l}-0.198 \\
{[0.289]}\end{array}$ & $\begin{array}{l}-0.0846 \\
{[0.218]}\end{array}$ & $\begin{array}{l}-0.178 \\
{[0.269]}\end{array}$ & $\begin{array}{l}-0.0547 \\
{[0.234]}\end{array}$ & $\begin{array}{l}0.0687 \\
{[0.246]}\end{array}$ & $\begin{array}{l}-0.173 \\
{[0.264]}\end{array}$ \\
\hline Average Temperature & & $\begin{array}{l}-0.283 \\
{[0.292]}\end{array}$ & $\begin{array}{c}-0.769^{* *} \\
{[0.346]}\end{array}$ & $\begin{array}{c}-0.834^{* *} \\
{[0.325]}\end{array}$ & $\begin{array}{c}-0.838^{* *} \\
{[0.345]}\end{array}$ & $\begin{array}{l}-0.707^{*} \\
{[0.357]}\end{array}$ & $\begin{array}{c}-0.817^{* *} \\
{[0.333]}\end{array}$ & $\begin{array}{c}-0.737^{* *} \\
{[0.345]}\end{array}$ & $\begin{array}{c}-0.792^{* *} \\
{[0.333]}\end{array}$ & $\begin{array}{c}-0.777^{* *} \\
{[0.357]}\end{array}$ & $\begin{array}{c}-0.692^{* *} \\
{[0.346]}\end{array}$ & $\begin{array}{c}-0.793^{* *} \\
{[0.327]}\end{array}$ \\
\hline Latitude & & $\begin{array}{c}-3.072^{* * *} \\
{[1.131]}\end{array}$ & $\begin{array}{l}2.797^{*} \\
{[1.523]}\end{array}$ & $\begin{array}{l}3.094^{*} \\
{[1.800]}\end{array}$ & $\begin{array}{l}2.928^{*} \\
{[1.609]}\end{array}$ & $\begin{array}{l}2.906^{*} \\
{[1.553]}\end{array}$ & $\begin{array}{l}3.263^{*} \\
{[1.778]}\end{array}$ & $\begin{array}{l}2.602^{* *} \\
{[1.323]}\end{array}$ & $\begin{array}{l}2.994^{*} \\
{[1.546]}\end{array}$ & $\begin{array}{l}2.652^{* *} \\
{[1.351]}\end{array}$ & $\begin{array}{l}2.728^{* *} \\
{[1.327]}\end{array}$ & $\begin{array}{l}2.934^{*} \\
{[1.527]}\end{array}$ \\
\hline Land Suitability & & $\begin{array}{c}-0.0983 \\
{[0.156]}\end{array}$ & $\begin{array}{l}0.0721 \\
{[0.131]}\end{array}$ & $\begin{array}{l}0.0856 \\
{[0.125]}\end{array}$ & $\begin{array}{l}0.0693 \\
{[0.129]}\end{array}$ & $\begin{array}{l}0.0610 \\
{[0.117]}\end{array}$ & $\begin{array}{l}0.0827 \\
{[0.124]}\end{array}$ & $\begin{array}{l}0.0582 \\
{[0.125]}\end{array}$ & $\begin{array}{l}0.0714 \\
{[0.121]}\end{array}$ & $\begin{array}{l}0.0534 \\
{[0.123]}\end{array}$ & $\begin{array}{l}0.0518 \\
{[0.113]}\end{array}$ & $\begin{array}{l}0.0747 \\
{[0.120]}\end{array}$ \\
\hline Share of Carboniferous Area & & & $\begin{array}{c}0.148 \\
{[0.229]}\end{array}$ & $\begin{array}{c}0.218 \\
{[0.258]}\end{array}$ & $\begin{array}{c}0.201 \\
{[0.231]}\end{array}$ & $\begin{array}{c}0.163 \\
{[0.200]}\end{array}$ & $\begin{array}{c}0.218 \\
{[0.258]}\end{array}$ & $\begin{array}{c}0.136 \\
{[0.216]}\end{array}$ & $\begin{array}{c}0.200 \\
{[0.240]}\end{array}$ & $\begin{array}{c}0.171 \\
{[0.217]}\end{array}$ & $\begin{array}{c}0.151 \\
{[0.188]}\end{array}$ & $\begin{array}{c}0.190 \\
{[0.244]}\end{array}$ \\
\hline Maritime Department & & & $\begin{array}{l}-0.0232 \\
{[0.0768]}\end{array}$ & $\begin{array}{l}-0.0114 \\
{[0.0888]}\end{array}$ & $\begin{array}{l}-0.0159 \\
{[0.0798]}\end{array}$ & $\begin{array}{l}-0.0507 \\
{[0.0837]}\end{array}$ & $\begin{array}{l}-0.0251 \\
{[0.0877]}\end{array}$ & $\begin{array}{l}-0.0540 \\
{[0.0827]}\end{array}$ & $\begin{array}{l}-0.0563 \\
{[0.0992]}\end{array}$ & $\begin{array}{l}-0.0553 \\
{[0.0834]}\end{array}$ & $\begin{array}{l}-0.0706 \\
{[0.0845]}\end{array}$ & $\begin{array}{l}-0.0540 \\
{[0.0883]}\end{array}$ \\
\hline Border Department & & & $\begin{array}{c}-0.243^{* * *} \\
{[0.0906]}\end{array}$ & $\begin{array}{c}-0.270^{* * *} \\
{[0.0991]}\end{array}$ & $\begin{array}{c}-0.258^{* *} \\
{[0.0984]}\end{array}$ & $\begin{array}{l}-0.225^{* *} \\
{[0.0964]}\end{array}$ & $\begin{array}{c}-0.265^{* *} \\
{[0.105]}\end{array}$ & $\begin{array}{c}-0.252^{* * *} \\
{[0.0861]}\end{array}$ & $\begin{array}{c}-0.272^{* * *} \\
{[0.0915]}\end{array}$ & $\begin{array}{c}-0.265^{* * *} \\
{[0.0929]}\end{array}$ & $\begin{array}{c}-0.235^{* * *} \\
{[0.0881]}\end{array}$ & $\begin{array}{c}-0.266^{* * *} \\
{[0.0959]}\end{array}$ \\
\hline Distance to Paris & & & $\begin{array}{c}0.00207^{* * *} \\
{[0.000444]}\end{array}$ & $\begin{array}{c}0.00223^{* * * *} \\
{[0.000488]}\end{array}$ & $\begin{array}{c}0.00205^{* * *} \\
{[0.000494]}\end{array}$ & $\begin{array}{c}0.00201^{* * *} \\
{[0.000448]}\end{array}$ & $\begin{array}{c}0.00224^{* * * *} \\
{[0.000467]}\end{array}$ & $\begin{array}{c}0.00209^{* * *} \\
{[0.000413]}\end{array}$ & $\begin{array}{c}0.00228^{* * *} \\
{[0.000440]}\end{array}$ & $\begin{array}{c}0.00208^{* * * *} \\
{[0.000440]}\end{array}$ & $\begin{array}{c}0.00204^{* * *} \\
{[0.000415]}\end{array}$ & $\begin{array}{c}0.00226^{* * *} \\
{[0.000419]}\end{array}$ \\
\hline Paris and Suburbs & & & $\begin{array}{c}0.912 \\
{[0.630]}\end{array}$ & $\begin{array}{l}0.177^{*} \\
{[0.100]}\end{array}$ & $\begin{array}{c}0.904 \\
{[0.600]}\end{array}$ & $\begin{array}{c}0.889 \\
{[0.572]}\end{array}$ & $\begin{array}{l}0.196^{*} \\
{[0.109]}\end{array}$ & $\begin{array}{c}0.898 \\
{[0.574]}\end{array}$ & $\begin{array}{c}0.190 \\
{[0.119]}\end{array}$ & $\begin{array}{c}0.890 \\
{[0.548]}\end{array}$ & $\begin{array}{l}0.882^{*} \\
{[0.529]}\end{array}$ & $\begin{array}{c}0.188 \\
{[0.122]}\end{array}$ \\
\hline Grooms who Signed their Marriage License, $1786-1790$ & & & & $\begin{array}{c}0.131 \\
{[0.199]}\end{array}$ & & & $\begin{array}{c}0.128 \\
{[0.216]}\end{array}$ & & $\begin{array}{c}0.104 \\
{[0.192]}\end{array}$ & & & $\begin{array}{l}0.0918 \\
{[0.203]}\end{array}$ \\
\hline University & & & & & $\begin{array}{c}0.132 \\
{[0.129]}\end{array}$ & & $\begin{array}{l}0.0122 \\
{[0.105]}\end{array}$ & & & $\begin{array}{l}0.0915 \\
{[0.125]}\end{array}$ & & $\begin{array}{l}-0.0265 \\
{[0.106]}\end{array}$ \\
\hline Urban Population in 1700 & & & & & & $\begin{array}{c}0.0511 \\
{[0.0319]}\end{array}$ & $\begin{array}{c}0.0152 \\
{[0.0206]}\end{array}$ & & & & $\begin{array}{c}0.0418 \\
{[0.0356]}\end{array}$ & $\begin{array}{l}0.00252 \\
{[0.0226]}\end{array}$ \\
\hline Observations & 85 & 85 & 85 & 79 & 85 & 85 & 79 & 85 & 79 & 85 & 85 & 79 \\
\hline & & & & & st stage: th & nstrumented & variable is $\mathrm{I}$ & mber of Steal & m Engines & & & \\
\hline Distance to Fresnes & & & & & & & & $\begin{array}{c}-0.00687^{* * *} \\
{[0.00250]}\end{array}$ & $\begin{array}{c}-0.00682^{* *} \\
{[0.00266]}\end{array}$ & $\begin{array}{c}-0.00702^{* * *} \\
{[0.00211]}\end{array}$ & $\begin{array}{c}-0.00585^{* *} \\
{[0.00245]}\end{array}$ & $\begin{array}{c}-0.00610^{* * *} \\
{[0.00230]}\end{array}$ \\
\hline $\begin{array}{l}\text { Deviation from Wheat Prices in } 1835-1838 \\
\text { (baseline 1810-1829) }\end{array}$ & & & & & & & & $\begin{array}{c}-1.633^{* * *} \\
{[0.481]}\end{array}$ & $\begin{array}{c}-1.608^{* * *} \\
{[0.527]}\end{array}$ & $\begin{array}{c}-1.571^{* * *} \\
{[0.493]}\end{array}$ & $\begin{array}{c}-1.463^{* * *} \\
{[0.511]}\end{array}$ & $\begin{array}{c}-1.401^{* *} \\
{[0.557]}\end{array}$ \\
\hline F-stat ( $1^{\text {st }}$ stage $)$ & & & & & & & & 13.126 & 10.072 & 14.403 & 8.658 & 8.043 \\
\hline Prob J-Stat & & & & & & & & 0.846 & 0.925 & 0.888 & 0.782 & 0.903 \\
\hline
\end{tabular}

Note: The explanatory variables except the dummies are in logarithm. The aerial distances are measured in kilometers. Robust standard errors are reported in brackets. $* * *$ indicates significance at the $1 \%$-level, ${ }^{* *}$ indicates significance at the $5 \%$-level, $*$ indicates significance at the $10 \%$-level. 
Table D.6: The effect of industrialization on the number of school buildings in 1863

\begin{tabular}{|c|c|c|c|c|c|c|c|c|c|c|c|c|}
\hline & $\begin{array}{l}\text { (1) } \\
\text { OLS }\end{array}$ & $\begin{array}{l}\text { (2) } \\
\text { OLS }\end{array}$ & $\begin{array}{l}\text { (3) } \\
\text { OLS }\end{array}$ & $\begin{array}{l}\text { (4) } \\
\text { OLS }\end{array}$ & $\begin{array}{l}\text { (5) } \\
\text { OLS }\end{array}$ & $\begin{array}{c}(6) \\
\text { OLS }\end{array}$ & $\begin{array}{l}\text { (7) } \\
\text { OLS }\end{array}$ & $\begin{array}{l}\text { (8) } \\
\text { IV }\end{array}$ & $\begin{array}{l}\text { (9) } \\
\text { IV }\end{array}$ & $\begin{array}{l}\text { (10) } \\
\text { IV }\end{array}$ & $\begin{array}{l}\text { (11) } \\
\text { IV }\end{array}$ & $\begin{array}{l}\text { (12) } \\
\text { IV }\end{array}$ \\
\hline & \multicolumn{12}{|c|}{ Schools per commune, 1863} \\
\hline Number of Steam Engines & $\begin{array}{c}0.0465 \\
{[0.0390]}\end{array}$ & $\begin{array}{c}0.121 * * * \\
{[0.0408]}\end{array}$ & $\begin{array}{l}0.109^{* * *} \\
{[0.0390]}\end{array}$ & $\begin{array}{c}0.0749^{* *} \\
{[0.0308]}\end{array}$ & $\begin{array}{c}0.0869^{* *} \\
{[0.0390]}\end{array}$ & $\begin{array}{c}0.0646^{* *} \\
{[0.0290]}\end{array}$ & $\begin{array}{l}0.0559^{*} \\
{[0.0306]}\end{array}$ & $\begin{array}{l}0.144^{* *} \\
{[0.0587]}\end{array}$ & $\begin{array}{l}0.133^{* *} \\
{[0.0600]}\end{array}$ & $\begin{array}{l}0.142^{* *} \\
{[0.0615]}\end{array}$ & $\begin{array}{c}0.0880 \\
{[0.0684]}\end{array}$ & $\begin{array}{c}0.120^{*} \\
{[0.0685]}\end{array}$ \\
\hline Average Rainfall & & $\begin{array}{l}-0.264 \\
{[0.301]}\end{array}$ & $\begin{array}{l}-0.249 \\
{[0.226]}\end{array}$ & $\begin{array}{l}-0.325 \\
{[0.283]}\end{array}$ & $\begin{array}{l}-0.203 \\
{[0.260]}\end{array}$ & $\begin{array}{l}0.0596 \\
{[0.268]}\end{array}$ & $\begin{array}{l}-0.176 \\
{[0.282]}\end{array}$ & $\begin{array}{l}-0.212 \\
{[0.208]}\end{array}$ & $\begin{array}{l}-0.235 \\
{[0.265]}\end{array}$ & $\begin{array}{l}-0.159 \\
{[0.231]}\end{array}$ & $\begin{array}{l}0.0591 \\
{[0.246]}\end{array}$ & $\begin{array}{l}-0.152 \\
{[0.258]}\end{array}$ \\
\hline Average Temperature & & $\begin{array}{l}-0.108 \\
{[0.303]}\end{array}$ & $\begin{array}{l}-0.547 \\
{[0.373]}\end{array}$ & $\begin{array}{c}-0.753^{* *} \\
{[0.326]}\end{array}$ & $\begin{array}{l}-0.650^{*} \\
{[0.356]}\end{array}$ & $\begin{array}{l}-0.450 \\
{[0.376]}\end{array}$ & $\begin{array}{c}-0.715^{* *} \\
{[0.325]}\end{array}$ & $\begin{array}{l}-0.519 \\
{[0.365]}\end{array}$ & $\begin{array}{c}-0.708^{* *} \\
{[0.332]}\end{array}$ & $\begin{array}{l}-0.589 \\
{[0.366]}\end{array}$ & $\begin{array}{l}-0.441 \\
{[0.357]}\end{array}$ & $\begin{array}{c}-0.689^{* *} \\
{[0.322]}\end{array}$ \\
\hline Latitude & & $\begin{array}{c}-2.805^{* *} \\
{[1.074]}\end{array}$ & $\begin{array}{l}1.921 \\
{[1.366]}\end{array}$ & $\begin{array}{c}2.085 \\
{[1.689]}\end{array}$ & $\begin{array}{c}2.118 \\
{[1.425]}\end{array}$ & $\begin{array}{c}2.090 \\
{[1.310]}\end{array}$ & $\begin{array}{c}2.397 \\
{[1.613]}\end{array}$ & $\begin{array}{l}1.757 \\
{[1.188]}\end{array}$ & $\begin{array}{c}1.977 \\
{[1.440]}\end{array}$ & $\begin{array}{c}1.841 \\
{[1.193]}\end{array}$ & $\begin{array}{l}1.983^{*} \\
{[1.108]}\end{array}$ & $\begin{array}{c}2.103 \\
{[1.352]}\end{array}$ \\
\hline Land Suitability & & $\begin{array}{l}-0.232 \\
{[0.173]}\end{array}$ & $\begin{array}{l}-0.0704 \\
{[0.157]}\end{array}$ & $\begin{array}{c}-0.00520 \\
{[0.138]}\end{array}$ & $\begin{array}{c}-0.0746 \\
{[0.151]}\end{array}$ & $\begin{array}{c}-0.0876 \\
{[0.130]}\end{array}$ & $\begin{array}{c}-0.00890 \\
{[0.122]}\end{array}$ & $\begin{array}{l}-0.0821 \\
{[0.149]}\end{array}$ & $\begin{array}{l}-0.0205 \\
{[0.132]}\end{array}$ & $\begin{array}{l}-0.0906 \\
{[0.144]}\end{array}$ & $\begin{array}{l}-0.0932 \\
{[0.123]}\end{array}$ & $\begin{array}{l}-0.0190 \\
{[0.123]}\end{array}$ \\
\hline Share of Carboniferous Area & & & $\begin{array}{c}0.128 \\
{[0.234]}\end{array}$ & $\begin{array}{c}0.174 \\
{[0.258]}\end{array}$ & $\begin{array}{c}0.208 \\
{[0.233]}\end{array}$ & $\begin{array}{c}0.152 \\
{[0.174]}\end{array}$ & $\begin{array}{c}0.163 \\
{[0.230]}\end{array}$ & $\begin{array}{c}0.119 \\
{[0.226]}\end{array}$ & $\begin{array}{c}0.155 \\
{[0.252]}\end{array}$ & $\begin{array}{c}0.178 \\
{[0.229]}\end{array}$ & $\begin{array}{c}0.145 \\
{[0.168]}\end{array}$ & $\begin{array}{c}0.140 \\
{[0.243]}\end{array}$ \\
\hline Maritime Department & & & $\begin{array}{l}-0.0139 \\
{[0.0728]}\end{array}$ & $\begin{array}{c}0.0217 \\
{[0.0846]}\end{array}$ & $\begin{array}{l}-0.00294 \\
{[0.0767]}\end{array}$ & $\begin{array}{l}-0.0568 \\
{[0.0832]}\end{array}$ & $\begin{array}{l}-0.00828 \\
{[0.0849]}\end{array}$ & $\begin{array}{l}-0.0398 \\
{[0.0778]}\end{array}$ & $\begin{array}{l}-0.0268 \\
{[0.0943]}\end{array}$ & $\begin{array}{l}-0.0424 \\
{[0.0805]}\end{array}$ & $\begin{array}{l}-0.0687 \\
{[0.0825]}\end{array}$ & $\begin{array}{l}-0.0394 \\
{[0.0832]}\end{array}$ \\
\hline Border Department & & & $\begin{array}{c}-0.221^{* * * *} \\
{[0.0817]}\end{array}$ & $\begin{array}{c}-0.239^{* *} \\
{[0.0938]}\end{array}$ & $\begin{array}{c}-0.244^{* * *} \\
{[0.0910]}\end{array}$ & $\begin{array}{c}-0.193^{* *} \\
{[0.0910]}\end{array}$ & $\begin{array}{c}-0.223^{* *} \\
{[0.0940]}\end{array}$ & $\begin{array}{c}-0.229^{* * *} \\
{[0.0772]}\end{array}$ & $\begin{array}{c}-0.241^{* * * *} \\
{[0.0856]}\end{array}$ & $\begin{array}{c}-0.250^{* * * *} \\
{[0.0859]}\end{array}$ & $\begin{array}{c}-0.199^{* *} \\
{[0.0818]}\end{array}$ & $\begin{array}{c}-0.227^{* *} \\
{[0.0890]}\end{array}$ \\
\hline Distance to Paris & & & $\begin{array}{c}0.00172^{* * *} \\
{[0.000409]}\end{array}$ & $\begin{array}{c}0.00181^{* * *} \\
{[0.000457]}\end{array}$ & $\begin{array}{c}0.00170^{* * *} \\
{[0.000464]}\end{array}$ & $\begin{array}{c}0.00163^{* * *} \\
{[0.000391]}\end{array}$ & $\begin{array}{c}0.00183^{* * *} \\
{[0.000422]}\end{array}$ & $\begin{array}{c}0.00174^{* * *} \\
{[0.000380]}\end{array}$ & $\begin{array}{c}0.00186^{* * *} \\
{[0.000410]}\end{array}$ & $\begin{array}{c}0.00173^{* * *} \\
{[0.000412]}\end{array}$ & $\begin{array}{c}0.00165^{* * *} \\
{[0.000366]}\end{array}$ & $\begin{array}{c}0.00185^{* * *} \\
{[0.000361]}\end{array}$ \\
\hline Paris and Suburbs & & & $\begin{array}{c}1.060 \\
{[0.783]}\end{array}$ & $\begin{array}{l}0.168^{* *} \\
{[0.0733]}\end{array}$ & $\begin{array}{c}1.048 \\
{[0.737]}\end{array}$ & $\begin{array}{c}1.023 \\
{[0.691]}\end{array}$ & $\begin{array}{l}0.209^{* *} \\
{[0.0884]}\end{array}$ & $\begin{array}{c}1.048 \\
{[0.717]}\end{array}$ & $\begin{array}{l}0.183^{* *} \\
{[0.0893]}\end{array}$ & $\begin{array}{c}1.033 \\
{[0.672]}\end{array}$ & $\begin{array}{c}1.019 \\
{[0.638]}\end{array}$ & $\begin{array}{c}0.203^{* *} \\
{[0.101]}\end{array}$ \\
\hline Grooms who Signed their Marriage License, $1786-1790$ & & & & $\begin{array}{c}0.00317 \\
{[0.179]}\end{array}$ & & & $\begin{array}{l}-0.0178 \\
{[0.179]}\end{array}$ & & $\begin{array}{l}-0.0252 \\
{[0.172]}\end{array}$ & & & $\begin{array}{c}-0.0468 \\
{[0.180]}\end{array}$ \\
\hline University & & & & & $\begin{array}{c}0.199 \\
{[0.140]}\end{array}$ & & & & & $\begin{array}{c}0.158 \\
{[0.135]}\end{array}$ & & $\begin{array}{l}-0.0233 \\
{[0.0976]}\end{array}$ \\
\hline Urban Population in 1700 & & & & & & $\begin{array}{c}0.0795^{* *} \\
{[0.0361]}\end{array}$ & $\begin{array}{c}0.0366^{* *} \\
{[0.0173]}\end{array}$ & & & & $\begin{array}{l}0.0739^{*} \\
{[0.0402]}\end{array}$ & $\begin{array}{c}0.0227 \\
{[0.0220]}\end{array}$ \\
\hline Observations & 85 & 85 & 85 & 79 & 85 & 85 & 79 & 85 & 79 & 85 & 85 & 79 \\
\hline & & & & & st stage: the & instrumented & variable is $\mathrm{N}$ & umber of Steal & n Engines & & & \\
\hline Distance to Fresnes & & & & & & & & $\begin{array}{c}-0.00687^{* * *} \\
{[0.00250]}\end{array}$ & $\begin{array}{c}-0.00682^{* *} \\
{[0.00266]}\end{array}$ & $\begin{array}{c}-0.00702^{* * *} \\
{[0.00211]}\end{array}$ & $\begin{array}{c}-0.00585^{* *} \\
{[0.00245]}\end{array}$ & $\begin{array}{c}-0.00610^{* * *} \\
{[0.00230]}\end{array}$ \\
\hline $\begin{array}{l}\text { Deviation from Wheat Prices in 1835-1838 } \\
\text { (baseline } 1810-1829 \text { ) }\end{array}$ & & & & & & & & $\begin{array}{c}-1.633^{* * *} \\
{[0.481]}\end{array}$ & $\begin{array}{c}-1.608^{* * *} \\
{[0.527]}\end{array}$ & $\begin{array}{c}-1.571^{* * *} \\
{[0.493]}\end{array}$ & $\begin{array}{c}-1.463^{* * *} \\
{[0.511]}\end{array}$ & $\begin{array}{c}-1.401^{* *} \\
{[0.557]}\end{array}$ \\
\hline F-stat $\left(1^{s t}\right.$ stage $)$ & & & & & & & & 13.126 & 10.072 & 14.403 & 8.658 & 8.043 \\
\hline Prob J-Stat & & & & & & & & 0.800 & 0.656 & 0.729 & 0.904 & 0.698 \\
\hline
\end{tabular}

Note: The explanatory variables except the dummies are in logarithm. The aerial distances are measured in kilometers. Robust standard errors are reported in brackets. ${ }^{* * *}$ indicates significance at the $1 \%$-level, $* *$ indicates significance at the $5 \%$-level, $*$ indicates significance at the $10 \%$-level. 


\section{Appendix E. Spatial Correlation}

Table E.1: The effect of industrialization on the number of teachers in 1840, accounting for spatial autocorrelation

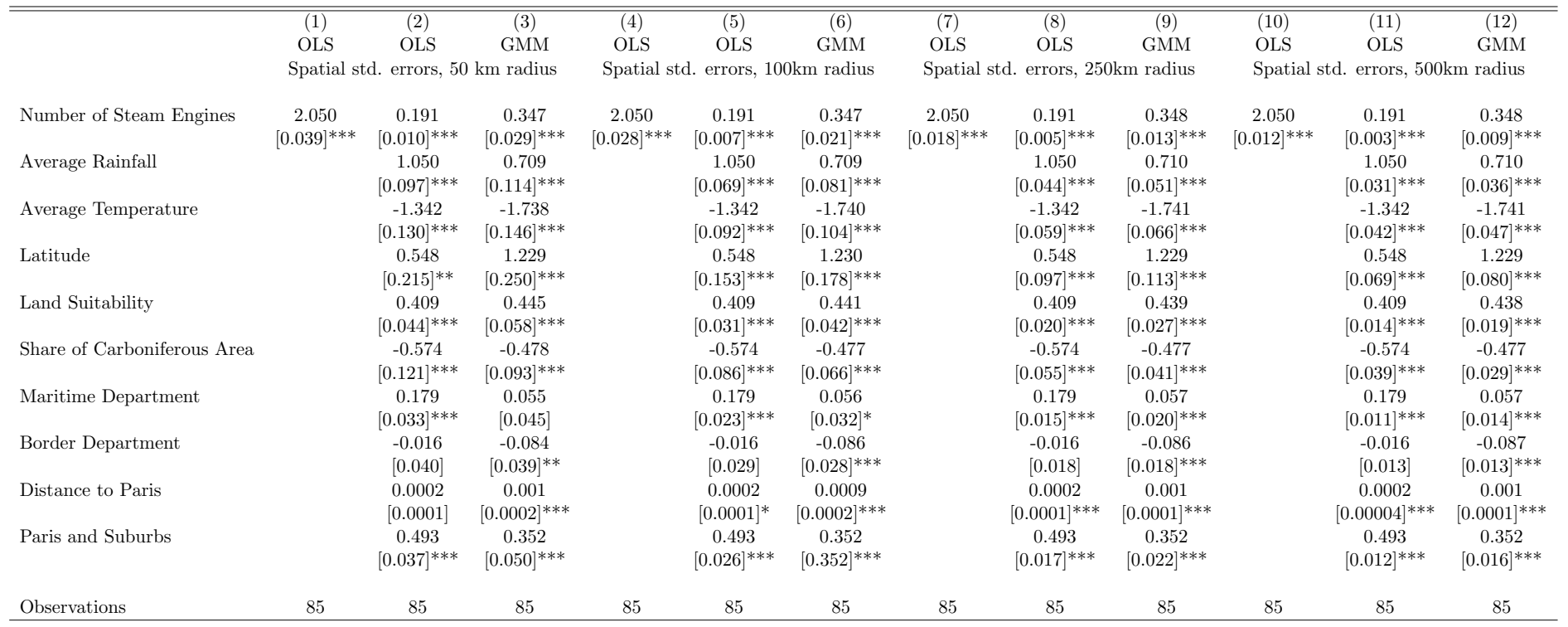

Note: The table reports spatial Conley (1999) standard errors with a radius of $100 \mathrm{~km}$ radii of $\mathbf{5 0} \mathbf{k m}, \mathbf{1 0 0} \mathbf{k m}, \mathbf{2 5 0} \mathbf{k m}$ and $\mathbf{5 0 0} \mathbf{k m}$. The explanatory variables except the dummies are in logarithm. The aerial distances are measured in kilometers. $* * *$ indicates significance at the $1 \%$-level, ** indicates significance at the $5 \%$-level, * indicates significance at the $10 \%$-level.

Table E.2: The effect of industrialization on the number of teachers in 1863, accounting for spatial autocorrelation

\begin{tabular}{|c|c|c|c|c|c|c|c|c|c|c|c|c|}
\hline & (1) & $(2)$ & $(3)$ & $(4)$ & $(5)$ & $(6)$ & $(7)$ & $(8)$ & $(9)$ & $(10)$ & $(11)$ & $(12)$ \\
\hline & OLS & OLS & GMM & OLS & OLS & GMM & OLS & OLS & GMM & OLS & OLS & GMM \\
\hline & \multicolumn{3}{|c|}{ Spatial std. errors, $50 \mathrm{~km}$ radius } & \multicolumn{3}{|c|}{ Spatial std. errors, $100 \mathrm{~km}$ radius } & \multicolumn{3}{|c|}{ Spatial std. errors, $250 \mathrm{~km}$ radius } & \multicolumn{3}{|c|}{ Spatial std. errors, $500 \mathrm{~km}$ radius } \\
\hline \multirow[t]{2}{*}{ Number of Steam Engines } & 2.224 & 0.201 & 0.301 & 2.224 & 0.201 & 0.301 & 2.224 & 0.201 & 0.301 & 2.224 & 0.201 & 0.301 \\
\hline & {$[0.045]^{* * *}$} & {$[0.008]^{* * *}$} & {$[0.023]^{* * *}$} & {$[0.032]^{* * *}$} & {$[0.005]^{* * *}$} & {$[0.016]^{* * *}$} & {$[0.020]^{* * *}$} & {$[0.003]^{* * *}$} & {$[0.010]^{* * *}$} & {$[0.014]^{* * *}$} & {$[0.002]^{* * *}$} & {$[0.007]^{* * *}$} \\
\hline \multirow[t]{2}{*}{ Average Rainfall } & & 0.699 & 0.334 & & 0.699 & 0.333 & & 0.699 & 0.332 & & 0.699 & 0.332 \\
\hline & & {$[0.087]^{* * *}$} & {$[0.147]^{* *}$} & & {$[0.062]^{* * *}$} & {$[0.105]^{* * *}$} & & {$[0.039]^{* * *}$} & {$[0.067]^{* * *}$} & & {$[0.028]^{* * *}$} & {$[0.048]^{* * *}$} \\
\hline \multirow[t]{2}{*}{ Average Temperature } & & -0.617 & -1.026 & & -0.617 & -1.029 & & -0.617 & -1.030 & & -0.617 & -1.031 \\
\hline & & {$[0.102]^{* * *}$} & {$[0.146]^{* * *}$} & & {$[0.073]^{* * *}$} & {$[0.105]^{* * *}$} & & {$[0.046]^{* * *}$} & {$[0.067]^{* * *}$} & & {$[0.033]^{* * *}$} & {$[0.047]^{* * *}$} \\
\hline \multirow[t]{2}{*}{ Latitude } & & 0.842 & 1.618 & & 0.842 & 1.621 & & 0.842 & 1.623 & & 0.842 & 1.624 \\
\hline & & {$[0.187]^{* * *}$} & {$[0.316]^{* * *}$} & & {$[0.133]^{* * *}$} & {$[0.227]^{* * *}$} & & {$[0.085]^{* * *}$} & {$[0.145]^{* * *}$} & & {$[0.060]^{* * *}$} & {$[0.103]^{* * *}$} \\
\hline \multirow[t]{2}{*}{ Land Suitability } & & 0.244 & 0.288 & & 0.244 & 0.287 & & 0.244 & 0.286 & & 0.244 & 0.286 \\
\hline & & {$[0.028]^{* * *}$} & {$[0.031]^{* * *}$} & & {$[0.020]^{* * *}$} & {$[0.022]^{* * *}$} & & {$[0.013]^{* * *}$} & {$[0.014]^{* * *}$} & & {$[0.009]^{* * *}$} & {$[0.010]^{* * *}$} \\
\hline \multirow[t]{2}{*}{ Share of Carboniferous Area } & & -0.063 & 0.045 & & -0.063 & 0.044 & & -0.063 & 0.043 & & -0.063 & 0.043 \\
\hline & & {$[0.073]$} & {$[0.074]$} & & {$[0.052]$} & {$[0.052]$} & & {$[0.033]^{*}$} & {$[0.033]$} & & {$[0.023]^{* * *}$} & {$[0.023]^{*}$} \\
\hline \multirow[t]{2}{*}{ Maritime Department } & & 0.159 & 0.082 & & 0.159 & 0.082 & & 0.159 & 0.081 & & 0.159 & 0.081 \\
\hline & & {$[0.020]^{* * *}$} & {$[0.020]^{* * *}$} & & {$[0.015]^{* * *}$} & {$[0.014]^{* * *}$} & & {$[0.009]^{* * *}$} & {$[0.009]^{* * *}$} & & {$[0.007]^{* * *}$} & {$[0.006]^{* * *}$} \\
\hline \multirow[t]{2}{*}{ Border Department } & & -0.108 & -0.162 & & -0.108 & -0.163 & & -0.108 & -0.163 & & -0.108 & -0.163 \\
\hline & & {$[0.028]^{* * *}$} & {$[0.025]^{* * *}$} & & {$[0.020]^{* * *}$} & {$[0.018]^{* * *}$} & & {$[0.012]^{* * *}$} & {$[0.011]^{* * *}$} & & {$[0.009]^{* * *}$} & {$[0.008]^{* * *}$} \\
\hline \multirow[t]{2}{*}{ Distance to Paris } & & 0.0002 & 0.001 & & 0.0002 & 0.001 & & 0.000 & 0.001 & & 0.0002 & 0.001 \\
\hline & & {$[0.0001]$} & {$[0.0002]^{* * *}$} & & {$[0.0001]$} & {$[0.0002]^{* * *}$} & & {$[0.014]^{* *}$} & {$[0.0001]^{* * *}$} & & {$[0.00004]^{* * *}$} & {$[0.0001]^{* * *}$} \\
\hline \multirow[t]{2}{*}{ Paris and Suburbs } & & 0.629 & 0.509 & & 0.629 & 0.510 & & 0.629 & 0.511 & & 0.629 & 0.511 \\
\hline & & {$[0.036]^{* * *}$} & {$[0.054]^{* * *}$} & & {$[0.025]^{* * *}$} & {$[0.038]^{* * *}$} & & {$[0.016]^{* * *}$} & {$[0.024]^{* * *}$} & & {$[0.011]^{* * *}$} & {$[0.017]^{* * *}$} \\
\hline Observations & 85 & 85 & 85 & 85 & 85 & 85 & 85 & 85 & 85 & 85 & 85 & 85 \\
\hline
\end{tabular}

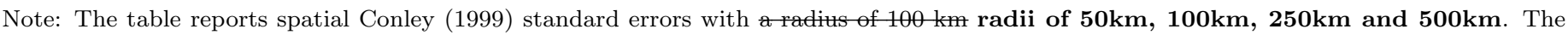
explanatory variables except the dummies are in logarithm. The aerial distances are measured in kilometers. $* * *$ indicates significance at the $1 \%$-level, ** indicates significance at the $5 \%$-level, * indicates significance at the $10 \%$-level. 
Table E.3: The effect of industrialization on the share of pupils in the population in 1840, accounting for spatial autocorrelation

\begin{tabular}{|c|c|c|c|c|c|c|c|c|c|c|c|c|}
\hline & (1) & $(2)$ & $(3)$ & (4) & $(5)$ & $(6)$ & $(7)$ & $(8)$ & (9) & $(10)$ & $(11)$ & $(12)$ \\
\hline & OLS & OLS & GMM & OLS & OLS & GMM & OLS & OLS & GMM & OLS & OLS & GMM \\
\hline & \multicolumn{3}{|c|}{ Spatial std. errors, $50 \mathrm{~km}$ radius } & \multicolumn{3}{|c|}{ Spatial std. errors, $100 \mathrm{~km}$ radius } & \multicolumn{3}{|c|}{ Spatial std. errors, $250 \mathrm{~km}$ radius } & \multicolumn{3}{|c|}{ Spatial std. errors, $500 \mathrm{~km}$ radius } \\
\hline \multirow[t]{2}{*}{ Number of Steam Engines } & 2.083 & 0.084 & 0.274 & 2.083 & 0.084 & 0.275 & 2.083 & 0.084 & 0.275 & 2.083 & 0.084 & 0.276 \\
\hline & {$[0.040]^{* * *}$} & {$[0.013]^{* * *}$} & {$[0.018]^{* * *}$} & {$[0.028]^{* * *}$} & {$[0.009]^{* * *}$} & {$[0.013]^{* * *}$} & {$[0.018]^{* * *}$} & {$[0.006]^{* * *}$} & {$[0.008]^{* * *}$} & {$[0.013]^{* * *}$} & {$[0.004]^{* * *}$} & {$[0.006]^{* * *}$} \\
\hline \multirow[t]{2}{*}{ Average Rainfall } & & 0.787 & 0.654 & & 0.787 & 0.644 & & 0.787 & 0.639 & & 0.787 & 0.637 \\
\hline & & {$[0.093]^{* * *}$} & {$[0.092]^{* * *}$} & & {$[0.066]^{* * *}$} & {$[0.065]^{* * *}$} & & {$[0.042]^{* * *}$} & {$[0.041]^{* * *}$} & & {$[0.030]^{* * *}$} & {$[0.029]^{* * *}$} \\
\hline \multirow[t]{2}{*}{ Average Temperature } & & -1.892 & -2.085 & & -1.892 & -2.095 & & -1.892 & -2.100 & & -1.892 & -2.101 \\
\hline & & {$[0.123]^{* * *}$} & {$[0.139]^{* * *}$} & & {$[0.088]^{* * *}$} & {$[0.099]^{* * *}$} & & {$[0.056]^{* * *}$} & {$[0.063]^{* * *}$} & & {$[0.039]^{* * *}$} & {$[0.044]^{* * *}$} \\
\hline \multirow[t]{2}{*}{ Latitude } & & 1.486 & 1.665 & & 1.486 & 1.687 & & 1.486 & 1.699 & & 1.486 & 1.703 \\
\hline & & {$[0.228]^{* * *}$} & {$[0.221]^{* * *}$} & & {$[0.163]^{* * *}$} & {$[0.157]^{* * *}$} & & {$[0.103]^{* * *}$} & {$[0.099]^{* * *}$} & & {$[0.073]^{* * *}$} & {$[0.070]^{* * *}$} \\
\hline \multirow[t]{2}{*}{ Land Suitability } & & 0.486 & 0.473 & & 0.486 & 0.475 & & 0.486 & 0.476 & & 0.486 & 0.477 \\
\hline & & {$[0.069]^{* * *}$} & {$[0.088]^{* * *}$} & & {$[0.050]^{* * *}$} & {$[0.063]^{* * *}$} & & {$[0.032]^{* * *}$} & {$[0.040]^{* * *}$} & & {$[0.022]^{* * *}$} & {$[0.029]^{* * *}$} \\
\hline \multirow[t]{2}{*}{ Share of Carboniferous Area } & & -0.840 & -0.808 & & -0.840 & -0.801 & & -0.840 & -0.798 & & -0.840 & -0.797 \\
\hline & & {$[0.101]^{* * *}$} & {$[0.078]^{* * *}$} & & {$[0.072]^{* * *}$} & {$[0.055]^{* * *}$} & & {$[0.046]^{* * *}$} & {$[0.035]^{* * *}$} & & {$[0.032]^{* * *}$} & {$[0.024]^{* * *}$} \\
\hline \multirow[t]{2}{*}{ Maritime Department } & & -0.043 & -0.196 & & -0.043 & -0.195 & & -0.043 & -0.194 & & -0.043 & -0.193 \\
\hline & & {$[0.053]$} & {$[0.058]^{* * *}$} & & {$[0.038]$} & {$[0.042]^{* * *}$} & & {$[0.024]^{*}$} & {$[0.026]^{* * *}$} & & {$[0.017]^{* *}$} & {$[0.019]^{* * *}$} \\
\hline \multirow[t]{2}{*}{ Border Department } & & 0.074 & 0.001 & & 0.074 & 0.003 & & 0.074 & 0.004 & & 0.074 & 0.004 \\
\hline & & {$[0.028]^{* * *}$} & {$[0.037]$} & & {$[0.020]^{* * *}$} & {$[0.027]$} & & {$[0.013]^{* * *}$} & {$[0.017]$} & & {$[0.009]^{* * *}$} & {$[0.012]$} \\
\hline \multirow[t]{2}{*}{ Distance to Paris } & & 0.0002 & 0.001 & & 0.0002 & 0.001 & & 0.0002 & 0.001 & & 0.0002 & 0.001 \\
\hline & & {$[0.0001]^{* *}$} & {$[0.0001]^{* * *}$} & & {$[0.0001]^{* * *}$} & {$[0.0001]^{* * *}$} & & {$[0.00004]^{* * *}$} & {$[0.0001]$} & & {$[0.00003]^{* * *}$} & {$[0.00004]^{* * *}$} \\
\hline \multirow[t]{2}{*}{ Paris and Suburbs } & & 0.157 & 0.039 & & 0.157 & 0.036 & & 0.157 & 0.034 & & 0.157 & 0.033 \\
\hline & & {$[0.039]^{* * *}$} & {$[0.055]$} & & {$[0.028]^{* * *}$} & {$[0.039]$} & & {$[0.018]^{* * *}$} & {$[0.025]$} & & {$[0.013]^{* * *}$} & {$[0.018]^{*}$} \\
\hline Observations & 85 & 85 & 85 & 85 & 85 & 85 & 85 & 85 & 85 & 85 & 85 & 85 \\
\hline
\end{tabular}

Note: The table reports spatial Conley (1999) standard errors with $100 \mathrm{~km}$ radii of $\mathbf{5 0 k m}, \mathbf{1 0 0} \mathbf{k m}, \mathbf{2 5 0} \mathbf{k m}$ and $\mathbf{5 0 0} \mathbf{k m}$. The explanatory variables except the dummies are in logarithm. The aerial distances are measured in kilometers. $* * *$ indicates significance at the $1 \%$-level, ** indicates significance at the $5 \%$-level, * indicates significance at the $10 \%$-level.

Table E.4: The effect of industrialization on the share of pupils in the population in 1863, accounting for spatial autocorrelation

\begin{tabular}{|c|c|c|c|c|c|c|c|c|c|c|c|c|}
\hline & (1) & $(2)$ & (3) & (4) & (5) & (6) & (7) & (8) & (9) & (10) & (11) & (12) \\
\hline & OLS & OLS & GMM & OLS & OLS & GMM & OLS & OLS & GMM & OLS & OLS & GMM \\
\hline & \multicolumn{3}{|c|}{ Spatial std. errors, $50 \mathrm{~km}$ radius } & \multicolumn{3}{|c|}{ Spatial std. errors, $100 \mathrm{~km}$ radius } & \multicolumn{3}{|c|}{ Spatial std. errors, $250 \mathrm{~km}$ radius } & \multicolumn{3}{|c|}{ Spatial std. errors, $500 \mathrm{~km}$ radius } \\
\hline \multirow[t]{2}{*}{ Number of Steam Engines } & 2.191 & 0.037 & 0.091 & 2.191 & 0.037 & 0.091 & 2.191 & 0.037 & 0.090 & 2.191 & 0.037 & 0.090 \\
\hline & {$[0.047]^{* * *}$} & {$[0.007]^{* * *}$} & {$[0.008]^{* * *}$} & {$[0.033]^{* * *}$} & {$[0.005]^{* * *}$} & {$[0.006]^{* * *}$} & {$[0.021]^{* * *}$} & {$[0.003]^{* * *}$} & {$[0.004]^{* * *}$} & {$[0.015]^{* * *}$} & {$[0.002]^{* * *}$} & {$[0.003]^{* * *}$} \\
\hline \multirow[t]{2}{*}{ Average Rainfall } & & 0.532 & 0.560 & & 0.532 & 0.551 & & 0.532 & 0.543 & & 0.532 & 0.539 \\
\hline & & {$[0.041]^{* * *}$} & {$[0.043]^{* * *}$} & & {$[0.029]^{* * *}$} & {$[0.033]^{* * *}$} & & {$[0.018]^{* * *}$} & {$[0.023]^{* * *}$} & & {$[0.013]^{* * *}$} & {$[0.016]^{* * *}$} \\
\hline \multirow[t]{2}{*}{ Average Temperature } & & -0.949 & -0.938 & & -0.949 & -0.947 & & -0.949 & -0.954 & & -0.949 & -0.956 \\
\hline & & {$[0.051]^{* * *}$} & {$[0.055]^{* * *}$} & & {$[0.037]^{* * *}$} & {$[0.040]^{* * *}$} & & {$[0.023]^{* * *}$} & {$[0.026]^{* * *}$} & & {$[0.016]^{* * *}$} & {$[0.019]^{* * *}$} \\
\hline \multirow[t]{2}{*}{ Latitude } & & 1.471 & 1.371 & & 1.471 & 1.393 & & 1.471 & 1.411 & & 1.471 & 1.418 \\
\hline & & {$[0.098]^{* * *}$} & {$[0.100]^{* * *}$} & & {$[0.070]^{* * *}$} & {$[0.077]^{* * *}$} & & {$[0.044]^{* * *}$} & {$[0.052]^{* * *}$} & & {$[0.031]^{* * *}$} & {$[0.037]^{* * *}$} \\
\hline \multirow[t]{2}{*}{ Land Suitability } & & 0.218 & 0.204 & & 0.218 & 0.206 & & 0.218 & 0.207 & & 0.218 & 0.208 \\
\hline & & {$[0.028]^{* * *}$} & {$[0.031]^{* * *}$} & & {$[0.020]^{* * *}$} & {$[0.022]^{* * *}$} & & {$[0.013]^{* * *}$} & {$[0.014]^{* * *}$} & & {$[0.009]^{* * *}$} & {$[0.010]^{* * *}$} \\
\hline \multirow[t]{2}{*}{ Share of Carboniferous Area } & & -0.483 & -0.491 & & -0.483 & -0.488 & & -0.483 & -0.486 & & -0.483 & -0.485 \\
\hline & & {$[0.058]^{* * *}$} & {$[0.045]^{* * *}$} & & {$[0.042]^{* * *}$} & {$[0.032]^{* * *}$} & & {$[0.026]^{* * *}$} & {$[0.021]^{* * *}$} & & {$[0.019]^{* * *}$} & {$[0.015]^{* * *}$} \\
\hline \multirow[t]{2}{*}{ Maritime Department } & & -0.037 & -0.074 & & -0.037 & -0.076 & & -0.037 & -0.077 & & -0.037 & -0.078 \\
\hline & & {$[0.030]$} & {$[0.028]^{* * *}$} & & {$[0.021]^{*}$} & {$[0.020]^{* * *}$} & & {$[0.014]^{* * *}$} & {$[0.013]^{* * *}$} & & {$[0.010]^{* * *}$} & {$[0.009]$} \\
\hline \multirow[t]{2}{*}{ Border Department } & & 0.017 & 0.003 & & 0.017 & 0.002 & & 0.017 & 0.002 & & 0.017 & 0.001 \\
\hline & & {$[0.012]$} & {$[0.016]$} & & {$[0.009]^{* *}$} & {$[0.012]$} & & {$[0.006]^{* * *}$} & {$[0.007]$} & & {$[0.004]^{* * *}$} & {$[0.005]$} \\
\hline \multirow[t]{2}{*}{ Distance to Paris } & & 0.0001 & 0.0002 & & 0.0001 & 0.0002 & & 0.0001 & 0.0002 & & 0.0001 & 0.0002 \\
\hline & & {$[0.00004]^{* * *}$} & {$[0.0001]^{* * *}$} & & {$[0.00003]^{* * *}$} & {$[0.00005]^{* * *}$} & & {$[0.00002]^{* * *}$} & {$[0.00003]^{* * *}$} & & {$[0.00001]^{* * *}$} & {$[0.00002]^{* * *}$} \\
\hline \multirow[t]{2}{*}{ Paris and Suburbs } & & 0.016 & -0.005 & & 0.016 & -0.007 & & 0.016 & -0.009 & & 0.016 & -0.009 \\
\hline & & {$[0.021]$} & {$[0.025]$} & & {$[0.015]$} & {$[0.018]$} & & {$[0.009]^{* * *}$} & {$[0.011]$} & & {$[0.007]^{* *}$} & {$[0.008]$} \\
\hline Observations & & 85 & 85 & 85 & 85 & 85 & 85 & 85 & 85 & 85 & 85 & 85 \\
\hline
\end{tabular}

Note: The table reports spatial Conley (1999) standard errors with a radius of $100 \mathrm{~km}$ radii of $50 \mathrm{~km}, \mathbf{1 0 0} \mathbf{k m}, \mathbf{2 5 0} \mathbf{k m}$ and $\mathbf{5 0 0 \mathrm { km }}$. The explanatory variables except the dummies are in logarithm. The aerial distances are measured in kilometers. $* * *$ indicates significance at the $1 \%$-level, ** indicates significance at the $5 \%$-level, * indicates significance at the $10 \%$-level. 
Table E.5: The effect of industrialization on the share of apprentices in the population in 1863, accounting for spatial autocorrelation

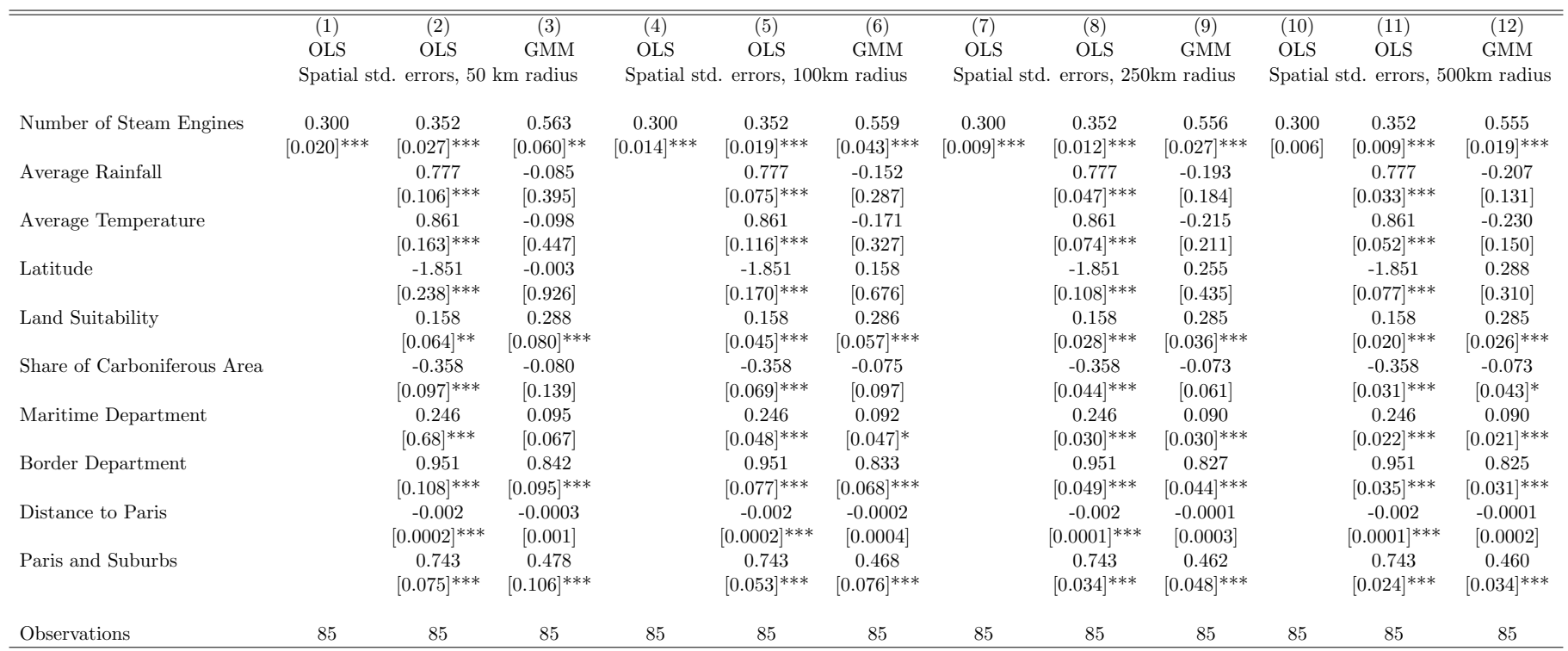

Note: The table reports spatial Conley (1999) standard errors with a radius of $100 \mathrm{~km}$. The explanatory variables except the dummies are in logarithm. The aerial distances are measured in kilometers. *** indicates significance at the $1 \%$-level, ** indicates significance at the $5 \%$-level, * indicates significance at the $10 \%$-level.

Table E.6: The effect of industrialization on the share of literate conscripts in 1859-1868, accounting for spatial autocorrelation

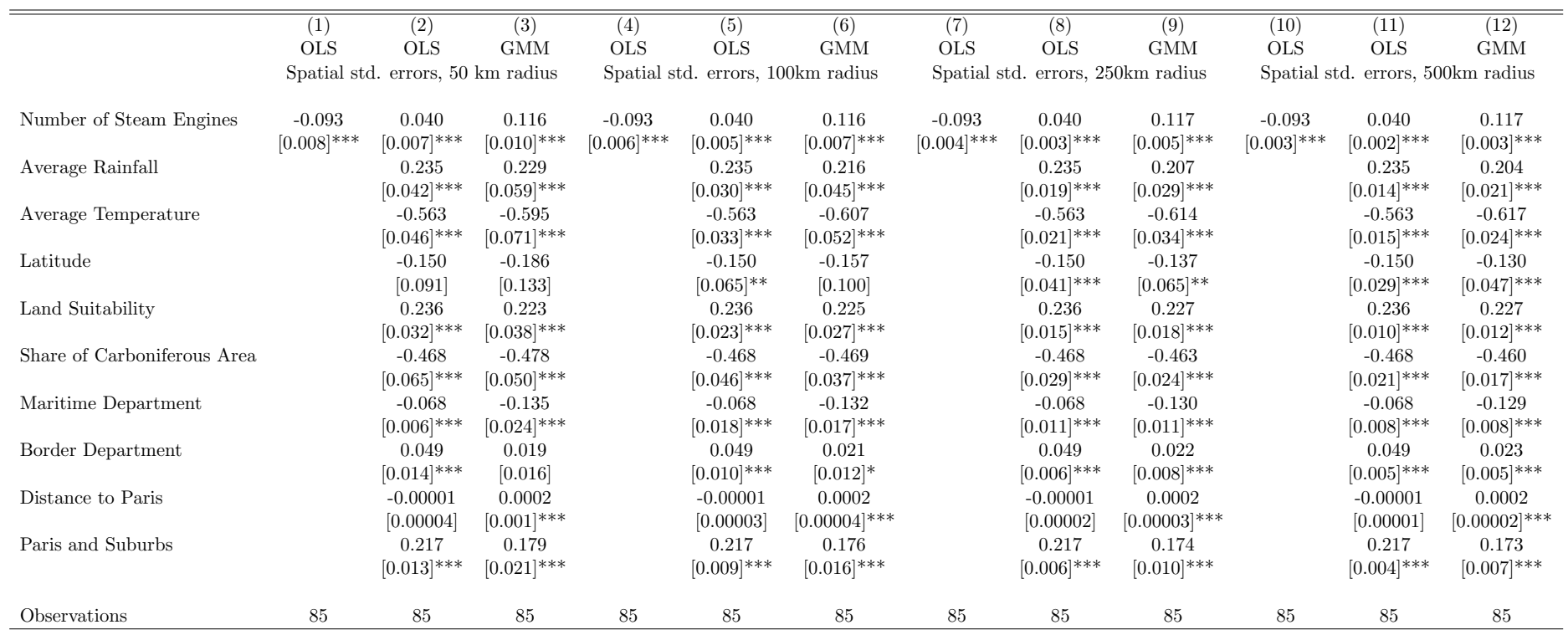

Note: The table reports spatial Conley (1999) standard errors with a radius of $100 \mathrm{~km}$. The explanatory variables except the dummies are in logarithm. The aerial distances are measured in kilometers. $* * *$ indicates significance at the $1 \%$-level, $* *$ indicates significance at the $5 \%$-level,

* indicates significance at the $10 \%$-level. 


\section{Appendix F. Variable Definitions and Sources}

\section{Dependent variables}

\section{Teachers.}

Teachers, 1840 and 1863. Number of teachers in primary schools. Source: Béaur, Gérard, and Béatrice Marin. 2011. La Statistique Générale de la France Présentation. L'Atelier du Centre de recherches historiques. http:acrh.revues.org/index2891.html.

\section{Pupils.}

Pupils, 1840 and 1863. Number of pupils enrolled in primary schools, per 10,000 inhabitants in the department. Source: Béaur, Gérard, and Béatrice Marin. 2011. La Statistique Générale de la France Présentation. L'Atelier du Centre de recherches historiques. http:acrh.revues.org/ index2891.html.

\section{Apprentices.}

Apprentices, 1863. Number of apprentices enrolled in apprentice schools, per 10,000 inhabitants in the department. Source: Ministère De l'Instruction Publique (1865).

\section{Literate conscripts}

Share of literate individuals among conscripts, 1847-1856 and 1859-1868. The average share of French army conscripts, i.e., 20-year-old men who reported for military service in the department where their father lived, who could read and write, computed over the 1847-1856 and 1859-1868 periods. Source: France. Ministère de la guerre (1791-1936) - Compte rendu sur le recrutement de l'armée.

\section{Public Spending on Education}

Total Public Spending on Primary Schooling per Inhabitant, 1855-1863. Source: Béaur, Gérard, and Béatrice Marin. 2011. La Statistique Générale de la France Présentation. L'Atelier du Centre de recherches historiques. http:acrh.revues.org/ index2891.html.

Commune Spending on Primary Schooling per Inhabitant, 1855-1863. Source: Béaur, Gérard, and Béatrice Marin. 2011. La Statistique Générale de la France Présentation. L'Atelier du Centre de recherches historiques. http:acrh.revues.org/ index2891.html.

Department Spending on Primary Schooling per Inhabitant, 1855-1863. Source: Béaur, Gérard, and Béatrice Marin. 2011. La Statistique Générale de la France Présentation. L'Atelier du Centre de recherches historiques. http:acrh.revues.org/ index2891.html.

Central State Spending on Primary Schooling per Inhabitant, 1855-1863. Source: Béaur, Gérard, and Béatrice Marin. 2011. La Statistique Générale de la France Présentation. L'Atelier du Centre de recherches historiques. http:acrh.revues.org/ index2891.html. 


\section{School buildings.}

Buildings, 1850 and 1863. Number of buildings per commune in each department. Source: Béaur, Gérard, and Béatrice Marin. 2011. La Statistique Générale de la France Présentation. L'Atelier du Centre de recherches historiques. http:acrh.revues.org/index2891.html.

\section{Explanatory variables}

Horse power of steam engines. This variable reports the number of steam Engines in the firms of each department, which is computed from the industrial survey carried out by the French government between 1839 and 1847. See Chanut et al. (2000) for details on the implementation of this survey.

Maritime department. This dummy variable takes the value one if a French department borders the coastline and zero otherwise.

Border department. This dummy variable takes the value one if a French department borders one of the foreign countries around France (Belgium, Luxembourg, Germany, Switzerland, Italy and Spain) and zero otherwise.

Distance to Paris. The great circle distance as "the crow flies" from Paris, the capital of France, to the administrative center of each department. This aerial distance is computed in kilometers.

Paris and suburbs. This dummy variable takes the value one for the three departments, i.e., Seine, Seine-et-Marne and Seine-et-Oise, which encompass Paris and its suburbs and zero otherwise.

Alsace-Lorraine. This dummy variable takes the value one for the Bas-Rhin, Haut-Rhin and Moselle departments and zero otherwise in all the regressions on post-WWI outcomes since these three departments were under German rule between 1871 and 1918.

Average rainfall. The average rainfall in $\mathrm{cm}^{3}$, reported at a half-degree resolution by Ramankutty et al. (2002), across the French departments.

Average temperature. The average temperature (in celsius), reported at a half-degree resolution by Ramankutty et al. (2002), across the French departments.

Latitude. The latitude of the centroid of each French department.

Land Suitability The land suitability index, reported at a half-degree resolution by Ramankutty et al. (2002), across the French departments.

Share of carboniferous area in department. The share of carboniferous area in each department. Source: Fernihough and O'Rourke (2014).

Share of Grooms who Signed their Wedding Licenses, 1786-1790. The share of grooms who signed their wedding licenses with their names over the 1786-1790 period (as opposed to those who marked it with a cross). Source: Béaur, Gérard, and Béatrice Marin. 2011. La Statistique Générale de la France Présentation. L'Atelier du Centre de recherches historiques. http:acrh.revues.org/ index2891.html.

University. Number of universities in 1700 in each department. Source: Bosker et al. (2013).

Urban population in 1700 (thousand of inhabitants). This variable reports the total population of the major urban centers, i.e., with more than 10,000 inhabitants, in each French department in 1700 using the data in Lepetit (1994, Appendix B). 


\section{Instrumental variables}

Distance to Fresnes sur Escaut. The great circle distance as "the crow flies" from Fresnes-surEscaut, where the first steam engine was operated in France in 1732, to the administrative center of each department. This aerial distance is computed in kilometers.

Deviation from Wheat Prices in 1835-1838 (baseline 1810-1829). Yearly wheat prices in each department. Source: Labrousse et al. (1970).

\section{Variables for robustness analysis}

\section{Economic development before 1815}

Market integration during the French Revolution. The number of external suppliers for each department in the 1790s for the following categories of products: cotton, hosiery, hardware, misc. production goods, misc. consumption goods, linen and hemp, wool and wool cloth, leather products hides and hats, iron, Food items, drinks, paper, wood for industry, fuel (wood and coal). Source: Daudin (2010).

Iron forges, 1789 and 1811. The number of iron forges in each department in 1789 and 1811. Source: Woronoff (1997).

Presence of iron forges, 1789 and 1811. The dummy variable takes the value 1 if there was at least one iron forge in a department in 1789. Source: Woronoff (1997).

\section{Human capital before 1820}

Share of Grooms who Signed their Wedding Licenses, 1686-1690 and 1816-1820. The share of grooms who signed their wedding licenses with their names over the 1686-1690 and 1816-1820 periods (as opposed to those who marked it with a cross). Source: Béaur, Gérard, and Béatrice Marin. 2011. La Statistique Générale de la France Présentation. L'Atelier du Centre de recherches historiques. http:acrh.revues.org/ index2891.html.

Encyclopédie subscribers. The number of subscribers to the Quarto edition of the Encyclopédie in the second half of the 18th century in the French towns aggregated at the department level. Source: Darnton (1973) and Squicciarini and Voigtländer (2015).

\section{Population density}

Population density, 1801, 1831 and 1861. Source: Béaur, Gérard, and Béatrice Marin. 2011. La Statistique Générale de la France Présentation. L'Atelier du Centre de recherches historiques. http:acrh.revues.org/index2891.html.

\section{Railroad connection}

Railroad connection to Paris in 1860. The dummy variable takes the value 1 if the administrative center of the department was connected to the railroad network in 1860. Source: Caron (1997). 


\section{Distance to London}

Distance to London. The great circle distance as "the crow flies" from London, the capital of England, to the administrative center of each department. This aerial distance is computed in kilometers.

\section{Past level of fertility}

Fertility, 1806. Fertility in each department, computed following the methodology of the Coale fertility index. 1806 is the earliest year available. Source: Bonneuil (1997).

\section{Distance to French cities}

Distance to Marseille. The great circle distance as "the crow flies" from Marseille to the administrative center of each department. This aerial distance is computed in kilometers.

Distance to Lyon. The great circle distance as "the crow flies" from Lyon to the administrative center of each department. This aerial distance is computed in kilometers.

Distance to Rouen. The great circle distance as "the crow flies" from Rouen to the administrative center of each department. This aerial distance is computed in kilometers.

Distance to Mulhouse. The great circle distance as "the crow flies" from Mulhouse to the administrative center of each department. This aerial distance is computed in kilometers.

Distance to Bordeaux. The great circle distance as "the crow flies" from Bordeaux to the administrative center of each department. This aerial distance is computed in kilometers.

Distance from Paris (weeks of travel). The time needed for a surface travel from Paris to the administrative center of each department measured in weeks of travel. Source: Özak (2013).

Distance from Marseille (weeks of travel). The time needed for a surface travel from Marseille to the administrative center of each department measured in weeks of travel. Source: Özak (2013).

Distance from Lyon (weeks of travel). The time needed for a surface travel from Lyon from Lyon to the administrative center of each department measured in weeks of travel. Source: Özak (2013).

Distance from Rouen (weeks of travel). The time needed for a surface travel from Rouen to the administrative center of each department measured in weeks of travel. Source: Özak (2013).

Distance from Mulhouse (weeks of travel). The time needed for a surface travel from Mulhouse to the administrative center of each department measured in weeks of travel. Source: Özak (2013).

Distance from Bordeaux (weeks of travel). The time needed for a surface travel from Bordeaux to the administrative center of each department measured in weeks of travel. Source: Özak (2013). 